\title{
Development of pigs
}

raised in a group housing system

for lactating sows and their litters

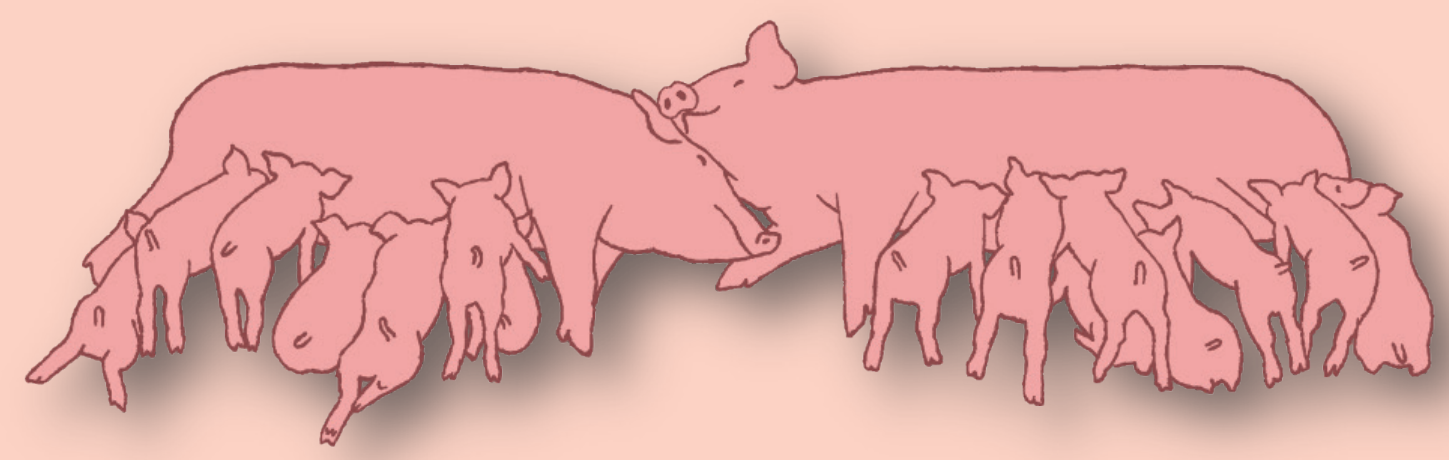

S.E. van Nieuwamerongen 


\section{Development of pigs raised in a group housing system for lactating sows and their litters}

S.E. van Nieuwamerongen 


\section{Thesis committee}

\section{Promotor}

Prof. Dr B. Kemp

Professor of Adaptation Physiology

Wageningen University \& Research

\section{Co-promotors}

Dr J.E. Bolhuis

Associate professor, Adaptation Physiology Group

Wageningen University \& Research

Dr N.M. Soede

Associate professor, Adaptation Physiology Group

Wageningen University \& Research

\section{Other members}

Prof. Dr W.J.J. Gerrits, Wageningen University \& Research

Prof. Dr A.E. Valros, University of Helsinki, Finland

Dr E. Baxter, Scotland's Rural College, Edinburgh, Scotland

Dr B.G. Meerburg, Wageningen University \& Research

This research was conducted under the auspices of the Graduate School of Wageningen Institute of Animal Sciences (WIAS) 


\section{Development of pigs raised in a group housing system for lactating sows and their litters}

\section{S.E. van Nieuwamerongen}

Thesis

submitted in fulfilment of the requirements for the degree of doctor

at Wageningen University

by the authority of the Rector Magnificus,

Prof. Dr A.P.J. Mol,

in the presence of the

Thesis Committee appointed by the Academic Board

to be defended in public

on Friday $19^{\text {th }}$ of May 2017

at 11 a.m. in the Aula. 
S.E. van Nieuwamerongen

Development of pigs raised in a group housing system for lactating sows and their litters, 270 pages.

$\mathrm{PhD}$ thesis, Wageningen University, Wageningen, the Netherlands (2017) With references, with summary in English

ISBN: 978-94-6343-139-2

DOI: 10.18174/409859 


\section{Abstract}

The aim of this thesis was to investigate the development of pigs that were raised in a newly developed group housing system for lactating sows and their litters, with a focus on the transition around weaning and performance later in life. The starting point of our multi-suckling (MS) system was the natural behaviour of pigs, and the system consisted of 5 farrowing pens connected to a communal area, which included a communal feeding area. Litters were grouped at 1 week of age. Compared with a conventional farrowing system (in which a sow and her piglets are housed in a pen in which the sow is confined individually in a crate), the MS system provided a more spacious and complex environment, with more social and physical enrichment. Before weaning, we found that MS piglets showed more feed-directed behaviour and less damaging behaviours, such as tail biting, than conventionally housed piglets. After weaning at 4 weeks of age, when housed in a more physically and socially enriched pen, MS-raised piglets showed a higher feed intake, a higher weight gain, more play behaviour, and less maladaptive behaviour than the conventionally raised piglets that were housed in a standard nursery pen. In a follow-up study, in which all pigs were housed under equal and enriched conditions after weaning, we found that piglets raised in the MS system had a higher feed utilisation and a lower carbohydrate absorption in an oral sugar absorption test in the early post-weaning phase. In a subsequent study, the same animals were used to investigate their social and cognitive development, using behaviour tests during which pigs competed for access to feed. We found few differences, but there were indications that the pigs raised pre-weaning in the MS system made more use of social information than the conventionally raised pigs. Lastly, we studied effects of different weaning procedures in the MS system (gradual weaning during 9 weeks of lactation vs. abrupt weaning at 4 weeks of age). Weaning seemed to have less impact for the pigs subjected to the gradual weaning treatment (reflected in weight gain and maladaptive behaviour). Also on the long term during the finishing phase (during which both groups were housed equally from 9 weeks of age), the pigs subjected to the gradual weaning treatment showed less maladaptive behaviour and had fewer body lesions than abruptly weaned pigs. To conclude, the multi-suckling system seems promising for improving pig performance, behaviour, and welfare, especially in combination with a more gradual weaning procedure during an extended lactation. 



\section{Table of contents}

Chapter 1 General introduction 9

Chapter 2 A review of sow and piglet behaviour and 31 performance in group housing systems for lactating sows

Chapter 3 Development of piglets raised in a new multi-litter 65 housing system vs. conventional single-litter housing until 9 weeks of age

Chapter 4 Effects of early life conditions on measures of piglet 95 performance around weaning

Chapter 5 Post-weaning social and cognitive performance of piglets raised pre-weaning either in a complex multisuckling group housing system or in a conventional system with a crated sow

Chapter 6 Gradual weaning improves performance and behaviour of pigs raised in a multi-suckling system

Chapter 7 General discussion

Summary

Samenvatting

Acknowledgements

About the author

Colophon 

CHAPTER 1

General introduction 


\subsection{Introduction}

This thesis will focus on the development of piglets raised in a newlydeveloped multi-suckling system, in which lactating sows are group housed together with their piglets. This thesis is part of a larger project with an overall goal to develop a commercially feasible group housing system for sows and their piglets, with the natural behaviour of pigs as the starting point. In the process of developing a housing system, many stakeholders should be considered, sometimes with conflicting interests, such as the sows, piglets, stockpersons, and consumers. Moreover, for a successful system performance, not only animal performance is important, but also aspects such as environmental impact and economical feasibility. These aspects are, however, beyond the scope of this thesis.

In this Chapter, the background that explains the rationale behind the development of the system is provided, as well as the potential impact of the system on piglet development. Given the starting point of the system, the natural behaviour of pigs around farrowing, lactation, and weaning is described first. Subsequently, a short history of pig farming in Western Europe is given, with a focus on housing for lactating sows and their piglets. Thereafter, the restriction of natural behaviours in current conventional farrowing systems is discussed in relation to welfare, health, and production problems for both sows and piglets. Next, recent developments of alternative farrowing systems are described, and a current perspective for group housing systems for sows and their piglets is provided. Lastly, the aim and outline of this thesis are presented.

\subsection{Natural behaviour of wild boar, feral pigs, and domesticated pigs in a natural environment}

Pigs were first domesticated around 9000 years ago (Larson and Fuller, 2014). Domestic pigs share a common ancestor with wild boar (Figure 1.1) and the behaviour of domestic pigs kept under (semi-)natural conditions still closely resembles that of wild boar (Jensen, 1986; Stolba and Wood-Gush, 1989; Petersen et al., 1990; Špinka et al., 2000). Feral pigs and wild boar live in home ranges up to $35 \mathrm{~km}^{2}$, consisting of diverse habitats (Singer et al., 1981; Dardaillon, 1986; Russo et al., 1997; Dexter, 1999; Saunders and McLeod, 1999). Pigs are opportunistic omnivores with a diverse diet and spend the 


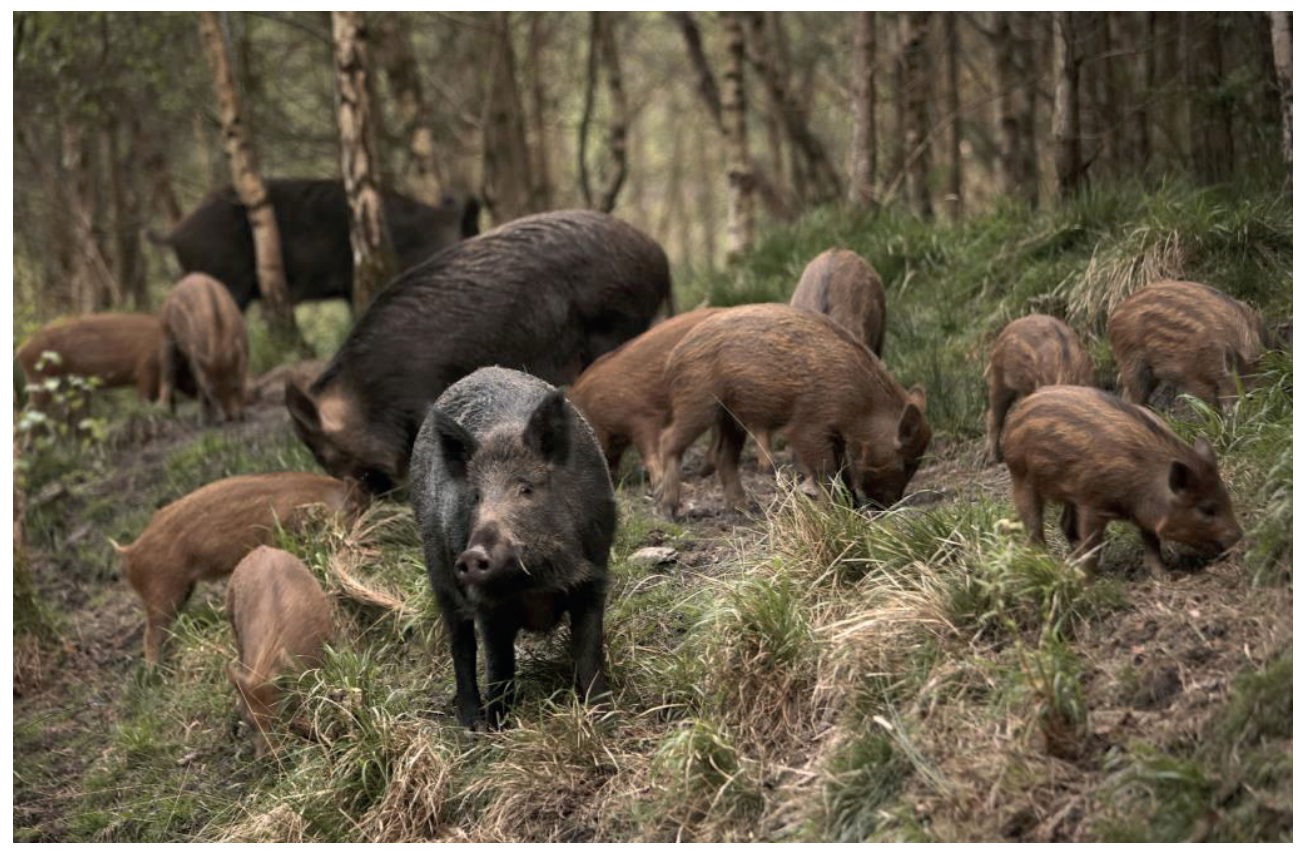

Figure 1.1. Wild boar in their natural habitat (source: Les Gibbon, Alamy, 2009).

majority of their active time foraging (Gundlach, 1968; Stolba and WoodGush, 1989; Schley and Roper, 2003). Their social structure is composed of family groups, generally consisting of several females and their offspring (Gundlach, 1968; Meynhardt, 1980; Jensen, 1986), which form long-lasting kin-directed bonds (Podgórski et al., 2014). The males separate from the group around 1 year of age and generally have a more solitary lifestyle (Meynhardt, 1980).

Around farrowing, a sow separates from the group and starts to look for a suitable site to build a nest, 1 to 2 days before giving birth to the piglets (Gundlach, 1968; Meynhardt, 1980; Jensen, 1986; Jensen et al., 1987; Stolba and Wood-Gush, 1989; Petersen et al., 1990; Stangel and Jensen, 1991; Jensen et al., 1993). Sows build a nest by forming a shallow hole which is covered by e.g. grass and branches (Stolba and Wood-Gush, 1989). The onset of nest building behaviour is hormonally regulated, but external factors such as weather conditions and availability of suitable materials, affect the completion of the nest (Jensen, 1989; Castrén et al., 1993; Jensen et al., 1993). Within the first few days after farrowing, the sow initially stays in the nest and forms a bond with her piglets, e.g. via nose contact (Meynhardt, 1980; Stangel and 
Jensen, 1991). Thereafter, the sow gradually starts leaving the nest, and the piglets subsequently gradually follow her when they are around 5 to 9 days old (Meynhardt, 1980; Jensen, 1986; Jensen and Redbo, 1987; Stangel and Jensen, 1991). The sow re-joins the group when the piglets are on average 9 to 10 days old and the litter gradually integrates with the other sows and litters (Jensen and Redbo, 1987; Stangel and Jensen, 1991).

The process by which piglets gain independence from the sow, resulting in weaning, is gradual and starts already early in lactation. In the first 10 days after farrowing, the initiation of nursing bouts shifts from the sows towards the piglets (Jensen et al., 1991) and the nature of contact between the sow and her litter changes, as e.g. nose contacts occur less frequently and contact via grunting increases (Jensen and Redbo, 1987). Moreover, nursing frequency gradually decreases over the course of lactation (Jensen, 1988; Jensen and Recén, 1989; Jensen and Stangel, 1992) and piglets gradually spend more time eating solid food components (Jensen, 1995). The piglets are fully weaned between 2 and 5 months of age (Meynhardt, 1980; Newberry and Wood-Gush, 1985; Jensen, 1986; Jensen and Recén, 1989). The age of complete weaning may vary within a litter (Jensen, 1995) and may depend on the season and on the parity of the sow (Newberry and Wood-Gush, 1985; Jensen and Recén, 1989).

\subsection{A short history of pig housing in Western Europe}

Since their domestication, pigs initially have been kept predominantly outdoors in a natural environment and were allowed to roam freely, e.g. in fields and forests, with ample possibilities to express their natural behavioural repertoire. Subsequent changes in pig housing are related to concurrent developments in e.g. human wealth, population size, and technical developments, but these aspects will not be considered in detail here. Up until the Middle Ages, shelters were mostly reserved for farrowing sows (Hartung, 2013). Figure 1.2 shows an example of such housing; the piglets were retained in a pen that only the sows could leave to visit a central area. This type of housing was already used around 2000 years ago (Columella, 1941). In the Middle Ages, pigs were often slaughtered before wintertime due to a lack of feed, and the remaining pigs were maintained on rather poor diets. One of the transitions towards higher productivity occurred during the agricultural revolution in the $17^{\text {th }}-18^{\text {th }}$ century. 


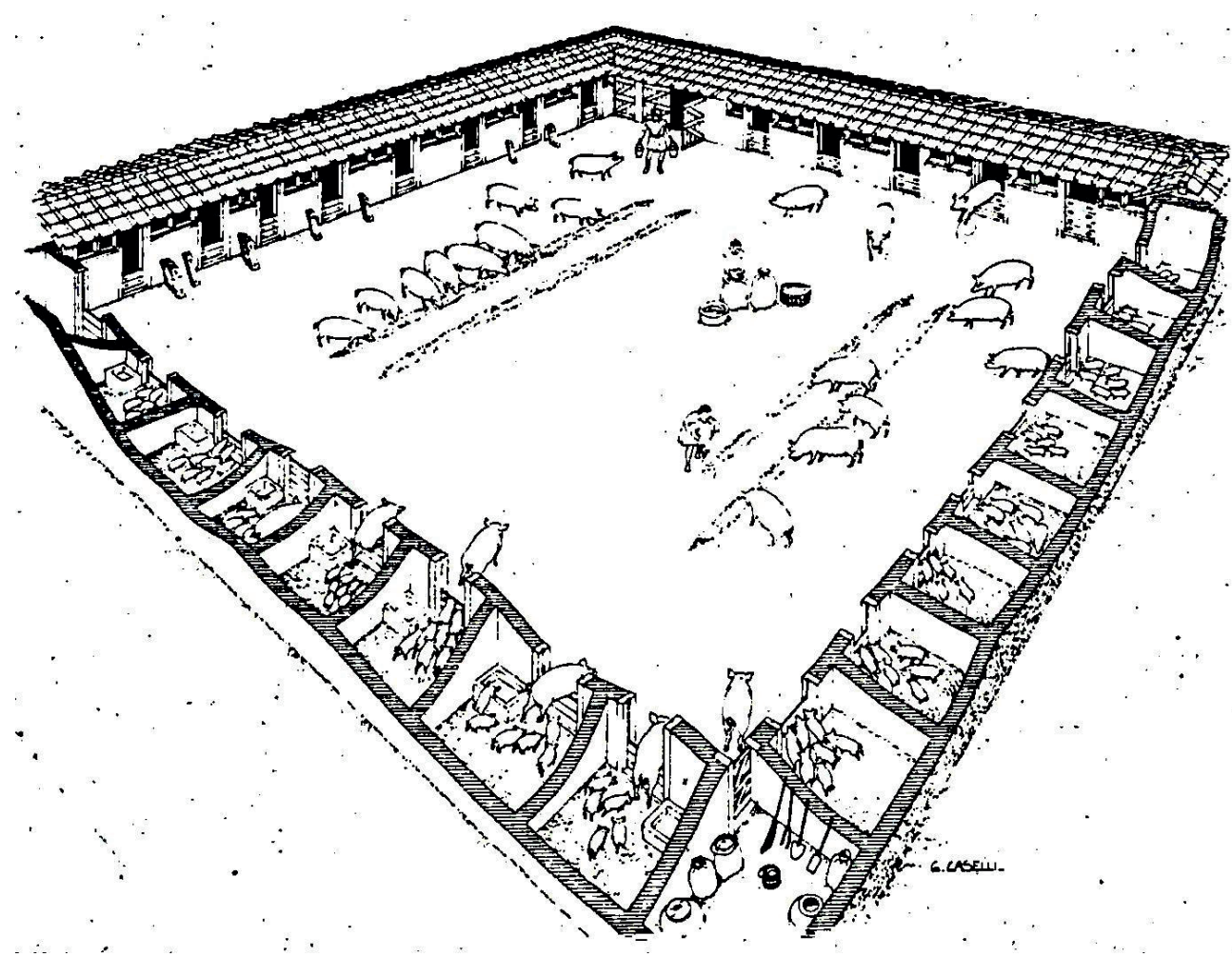

Figure 1.2. Roman pig farming system around 2000 years ago (Settis and Bonamici, 1985).

One of the major milestones of this revolution encompassed the integration of crop rotation systems with animal production. More animals could be kept year-round, as land was used more efficiently and it became possible to systematically produce animal feed and prepare pastures designated specifically for livestock. Driven by an increasing demand for food related to an increasing population size, methods to further increase animal production were investigated with the introduction of modern science in agriculture in the $18^{\text {th }}$ century (Hartung, 2013). Up until around the 1950s, pigs were kept mainly in combinations of indoor and outdoor housing (Fishwick, 1949; Dommerhold, 1951; Fraser et al., 2001). An example of such housing is shown in Figure 1.3; sows farrowed in sheltered individual pens, and a small group of sows and their litters together had access to a pasture. Piglets were generally weaned at 8 weeks of age, although later weaning around 12 weeks of age also occurred (Fishwick, 1949; Dommerhold, 1951). After the Second World War, there was a move towards more intensive indoor housing of pigs, due to a further 


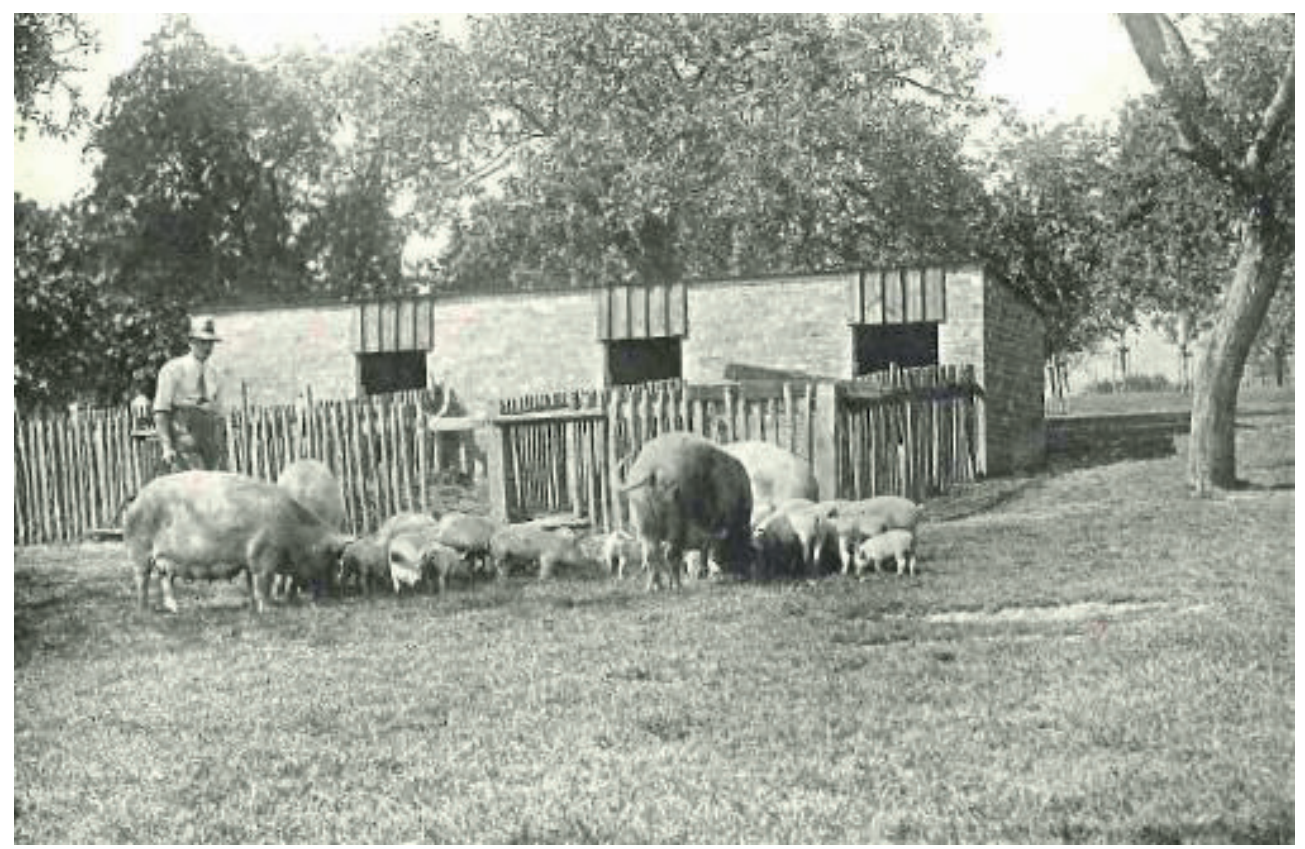

Figure 1.3. A group of sows and their piglets with an indoor shelter and access to a yard (Fishwick, 1949).

demand for more efficient animal production. The need for such systems is illustrated by a quote from Fishwick (1949): "The keynote of post-war management lies in the fact that foods and labour are likely to be in short supply and to command comparatively high prices for some years to come. As a consequence it will be necessary to employ methods designed to secure the greatest possible economy in the use of food and labour and to utilise labour-saving buildings and equipment to the maximum extent." Around the 1960s-1970s, intensive systems started to be common, which were characterised by year-round indoor housing, a high stocking density, and a low labour input due to a high level of mechanisation and automation of routine tasks such as providing the animals with feed and water, and removing dung (Fraser et al., 2001; Hartung, 2013). For gestating sows, 2 major types of housing consisted of tether stalls, in which pigs were tied by the neck or girth in a partial stall, and closed crates that housed individual sows (Barnett et al., 2001). For sows and their piglets around farrowing and during lactation, the farrowing crate (Figure 1.4) became popular, which confines an individual sow in a way that she cannot turn around, with the piglets surrounding her in a pen. The farrowing crate was designed to require relatively little space, facilitate easy management for the farmer, and limit accidental crushing of the piglets 


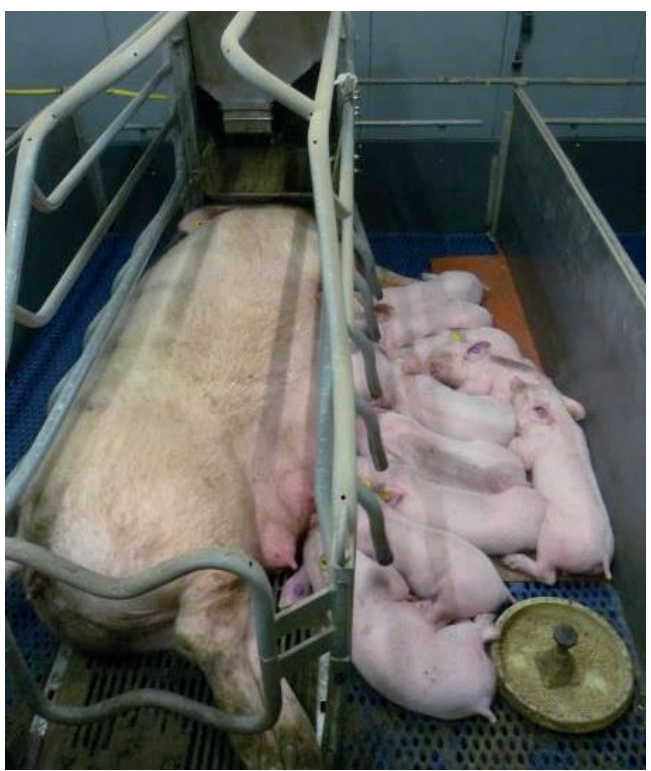

Figure 1.4. A sow confined in a farrowing crate with her litter. when the sow lies down (Barnett et al., 2001; Fraser et al., 2001). To increase production, not only changes in housing occurred, but also in management and genetic selection. For instance, a lower weaning age and selection for larger litters increased the number of piglets produced per sow per year (Fraser, 1978; Legault, 1985).

With the use of more intensive production systems, a sufficient food supply was secured for the majority of the population and peoples' concerns shifted more towards the welfare of the intensively kept animals (Hartung, 2013). The publication of the book 'Animal Machines' in 1964 played a major role in increasing public awareness of animal welfare issues in 'factory farming' (Harrison, 2013). Around that time, also the Brambell report appeared (Brambell, 1965), in which recommendations were given for legislation to improve animal welfare. These largely influenced later European legislation (Rushen, 2008; Veissier et al., 2008), which was characterised by limiting harmful procedures and providing animals more space, more opportunity for social contacts, a balanced diet, and an enriched environment (Veissier et al., 2008). Specifically for pigs, tethering of sows was phased out from 1996 and prohibited from 2006 onwards (Council Directive 91/630/EEC). More recently, individual confinement of sows during the major part of gestation was phased out from 2003 and prohibited from 2013 onwards (Council Directive $2001 / 88 / E C)$. During farrowing and lactation, however, around $95 \%$ of sows in Europe are still housed individually in a farrowing crate (Barnett et al., 2001; Baxter et al., 2012) during a lactation period of 3 to 4 weeks, with mainly slatted flooring, no bedding or nesting material, and minimal enrichment. This system, hereafter referred to as the 'conventional' farrowing system, is currently a topic of societal concern (Baxter et al., 2012). 


\subsection{Issues related to the current conventional farrowing system}

Around farrowing and lactation, the physical and social environment of a conventional farrowing system (including aspects related to management) largely differs from a natural situation. Although this provides several benefits for the animals, such as continuous availability of water and protection from extreme weather conditions, the expression of natural behaviour is restricted. As is explained in this section, this can result in welfare, health, and production problems for both sows and piglets.

Regarding the sows, for instance, nest building behaviour prior to farrowing is restricted by a lack of space and suitable nesting material (Andersen et al., 2014; Yun et al., 2014a). Domestication has not eliminated the motivation to build a nest (Gustafsson et al., 1999) and crated sows may show behaviours such as teeth grinding, biting of pen fixtures, and frequent postural changes in response to thwarted nest building behaviour (Wischner et al., 2009; Andersen et al., 2014; Yun et al., 2015). Due to a lack of feedback from completing a suitable nest, these restless behaviours may continue during the farrowing process. Mediated by hormonal alterations, restricting the expression of nest building behaviour may result in a prolonged parturition and a negative impact on piglets' colostrum intake and sows' maternal behaviour, resulting in e.g. less careful lying down behaviour (Wischner et al., 2009; Yun et al., 2014a; Yun et al., 2014b; Yun and Valros, 2015). Altogether, this can jeopardise piglet survival. Moreover, continuous confinement in the relatively barren environment and in close proximity of the piglets may be stressful for the sows, and restricts maternal behaviour by limiting free interaction between the sow and her litter. Especially in late lactation, sows may experience stress in a conventional farrowing system, as indicated by increased cortisol levels (Cronin et al., 1991; Jarvis et al., 2006). In addition, the expression of social behaviour is restricted due to lack of contact with other sows and their litters, which may impair welfare, especially in later lactation (Barnett et al., 2001). Furthermore, foraging and exploratory behaviours are restricted by the lack of enrichment and the concentrated diet which is delivered at relatively large time intervals. This may result in oral stereotypies and gastro-intestinal lesions (Bergeron et al., 2006). Lastly, sows may experience physical discomfort due to confinement and hard flooring, which may result in injuries to e.g. the legs, shoulders and udder (Bonde et al. 2004). 
Also piglets' natural behaviours are restricted in conventional husbandry, which affects their pre-weaning development and subsequent post-weaning performance. At weaning, several abrupt changes occur simultaneously; piglets are separated from the sow, switch to a different diet, are relocated to a nursery, and are often mixed with unfamiliar piglets (Weary et al., 2008). These stressful changes occur at an age when piglets are still immature in terms of the development of e.g. their immune system and gastro-intestinal tract (King et al., 2003; Miller and Slade, 2003), and result in a variety of postweaning problems. Piglets often have a low feed intake after weaning, which contributes to impaired intestinal function, characterised by suboptimal digestion and absorption of nutrients and a compromised barrier function which facilitates bacteria and toxins to cross the gut epithelium. Consequently, piglets often have diarrhoea and experience a growth check (VenteSpreeuwenberg and Beynen, 2003). The difficulties in coping with weaning may be reflected in behaviours such as increased restlessness, belly-nosing, and damaging oral manipulation of pen mates, and mixing of unfamiliar pigs elicits aggression (Fraser, 1978; Dybkjær, 1992). The way piglets endure the weaning process can have long-term consequences for their performance (Campbell et al., 2013).

For a successful weaning process, piglets need to gain independence from their mother before weaning, by decreasing their milk intake and increasing intake from other nutrient sources, such as liquid or solid piglet feed. In a conventional system, however, the sow and her litter are housed in close proximity during the entire lactation period. This may hamper a decrease in piglets' milk intake as it is e.g. difficult for the sow to evade piglets' nursing requests. Additionally, conventional weaning occurs at an age at which piglets still rely on milk as their main source of nutrients (Weary et al., 2008). Thus, pre-weaning piglet feed intake is generally low, and also varies greatly between individuals (Pajor et al., 1991; Bøe and Jensen, 1995; Bruininx et al., 2002). Besides the relatively young age, the lack of experience with piglet feed may be related to limited opportunities for piglets to learn how to eat from their mother and other piglets by observation and participation (Morgan et al., 2001; Oostindjer et al., 2011). Piglet feed is generally provided in a feeder that can be accessed only by a few piglets simultaneously, and which is located separately from the sow feeder. Additionally, the relatively barren environment lacks substrates, such as straw, which can stimulate the development of foraging- 
related behaviours (Oostindjer et al., 2014). The absence of suitable rooting materials also frustrates the piglets' natural behavioural need to explore. This can lead to redirected damaging behaviours, such as tail biting (Studnitz et al., 2007), which is a serious welfare and economical issue in conventional husbandry (e.g. Harley et al., 2014).

For a successful transition around weaning, piglets also need to be able to cope with the post-weaning changes in their social and physical environment. Space and environmental complexity, which are limited in a conventional farrowing system, are important for piglets to express a range of behaviours, such as play, threatening, and avoidance (McGlone and Curtis, 1985; Weng et al., 1998), which are important for the development of social skills. Also, the lack of social contact with other litters before weaning may hamper piglets' social development, as e.g. post-weaning aggression to familiar (Hessel et al., 2006) and unfamiliar (Kanaan et al., 2012) piglets may be increased. The lack of social and physical enrichment may also impair cognitive abilities (e.g. Sneddon et al., 2000; Grimberg-Henrici et al., 2016) and impair the piglets' ability to cope with a change in environment (Hillmann et al., 2003). Thus, piglets reared in a conventional system may have a suboptimal adaptive capacity to deal with stressful situations.

To conclude, the combination of management aspects around weaning (i.e. the combination of simultaneous abrupt changes at a relatively young age) and several aspects of housing before and after weaning (i.e. the physical and social environment), result in impaired pre-weaning and post-weaning piglet development in a conventional system.

\subsection{Development of alternative farrowing systems}

The importance of meeting animals' behavioural needs in animal production is increasingly recognised (Lidfors et al., 2005; Thornton, 2010; Hartung, 2013). The standard use of farrowing crates is currently prohibited in Switzerland, Sweden, and Norway. In other European countries, however, alternatives to conventional housing, such as organic farming, generally exist only as a niche market. For instance, in the Netherlands only $2 \%$ of pig farms are organic (CBS, 2014). Over the past decades, a variety of alternative farrowing systems has been investigated, which provide more freedom of movement for the sow and generally aim to be more welfare friendly. Systems have been studied in 
which sows are crated only temporarily when the risk of piglet crushing is highest, i.e. until about 3 days after farrowing (e.g. Lambertz et al., 2015). Furthermore, systems have been developed in which the sow has freedom of movement during the complete farrowing and lactation phase (e.g. Baxter et al., 2015). Lastly, there are systems that, besides offering more space, provide a more natural social environment by allowing mingling between multiple sows and/or litters before weaning. Examples of such systems include get-away systems, in which the sows can leave their piglets to meet in a communal area (cf. Figure 1.2), and multi-suckling systems, in which sows are group-housed together with their piglets. These get-away and multi-suckling systems are described in more detail in Chapter 2.

From the alternative systems, multi-suckling (MS) systems best resemble the social organisation seen in nature, in which sows farrow individually and form family groups during lactation. The possibility to interact with multiple sows and litters in the more spacious and complex environment of an MS system may provide piglets, for instance, more possibilities for social learning (e.g. of eating behaviour), development of social skills (e.g. through play and agonistic behaviour), and enables more balanced interactions between sows and piglets, which can stimulate piglets' independence (e.g. sows have more space to avoid the piglets). Furthermore, MS systems have the potential to further stimulate the expression of natural behaviours of sows and piglets if they include unconfined farrowing, substrates that can serve e.g. as bedding, nesting, and rooting material, and the possibility to wean the piglets more gradually at an older age, by e.g. enabling the sows to separate from the piglets during late lactation. Combined, these aspects may benefit sow welfare and improve piglets' pre-weaning and post-weaning development, and may contribute to alleviating the variety of post-weaning issues that piglets generally experience in a conventional system.

Although MS systems were used more commonly in the past and have largely been replaced by the current conventional farrowing system, the combination of the recent legislative change to group housing of sows during gestation and the issues associated with the current farrowing system -concerning sow and piglet health, welfare, and production performance- provides a new perspective for the use of MS systems. Despite the potential benefits of MS systems, some knowledge gaps regarding piglet development remain. Studies on MS systems 
in the past decades mostly included measurements of piglet mortality, (cross-)suckling, feed intake, weight gain, and aggression. After weaning, studies mainly focussed on piglets' production performance. Other aspects, such as the development of the gastro-intestinal tract, the immune system, cognitive function, stress physiology, and maladaptive behaviour have received little attention, especially around transitions such as weaning, but also on the longer term.

\subsection{Aim and outline of this thesis}

The main aim of this thesis is to investigate the development of piglets that are raised in a multi-suckling system, with a focus on the transition around weaning and performance later in life. To this end, first a literature review focussing on potential success- and risk factors associated with group housing systems for lactating sows is presented in Chapter 2, followed by experiments focussing on development of piglets raised in the new system in Chapters 3 to 6 . The key features in system design and management are based on the findings of the review in Chapter 2; the MS system has 5 pens with nesting material in which sows can farrow individually without being crated, connected with a communal area (Figure 1.5). The latter is divided in an area for general activity and resting, a communal feeding area (where sows and piglets can eat together), and a dunging area. Additionally, enrichment is provided throughout the system in the form of straw, hessian sacks, and ropes. Using the literature review (Chapter 2), knowledge gaps are identified that give direction to the experiments conducted in Chapters 3 to 6 .

The first experiment in Chapter 3 describes development of piglets in a system comparison from birth until 9 weeks of age. Piglets were weaned at 4 weeks of age, and they were housed either in the MS system followed by post-weaning housing in an enriched pen with 40 piglets, or in a conventional farrowing system followed by post-weaning housing in groups of 10 litter-mates in a standard nursery pen. It was hypothesised that piglets raised in the MS system would, overall, show an improved pre-weaning and post-weaning development compared with the conventionally reared piglets.

To further improve system and animal performance, a new version of the MS system was studied in Chapters 4, 5 and 6. This system contained 2 MS units with the same key features as the previous system. In Chapters 4 and 5, studies 
are described with a similar weaning age and contrast in pre-weaning housing conditions as in Chapter 3. To focus on effects of pre-weaning housing conditions, post-weaning housing was equal for both treatment groups, unlike in Chapter 3. The study in Chapter 4 aimed to gain more insight in the impact of the 2 different pre-weaning housing conditions on piglets' performance around weaning, including measures of intestinal function. It was hypothesised that the MS system would promote foraging-related behaviours and early preweaning experience with solid feed due to e.g. more opportunities for social learning in the communal feeding area, which may gradually prepare the intestinal tract for digestion and absorption of solid feed and thereby ease the transition to the post-weaning diet. Chapter 5 focusses on the post-weaning social and cognitive performance of the piglets raised in the MS system versus the conventional system, using behaviour tests. It was hypothesised that the physically and socially more enriched MS system would enhance the development of piglets' social skills and cognitive performance, by providing more environmental complexity. Finally, in Chapter 6 the added value of a more gradual weaning process in an MS system is evaluated. Development from birth until slaughter was studied in 2 groups that differed in treatment between 4 and 9 weeks of age. From 0 to 4 weeks of age, both groups were housed in the MS system. Thereafter, pigs were either subjected to abrupt weaning at 4 weeks of age and subsequently housed in a nursery, or kept in the

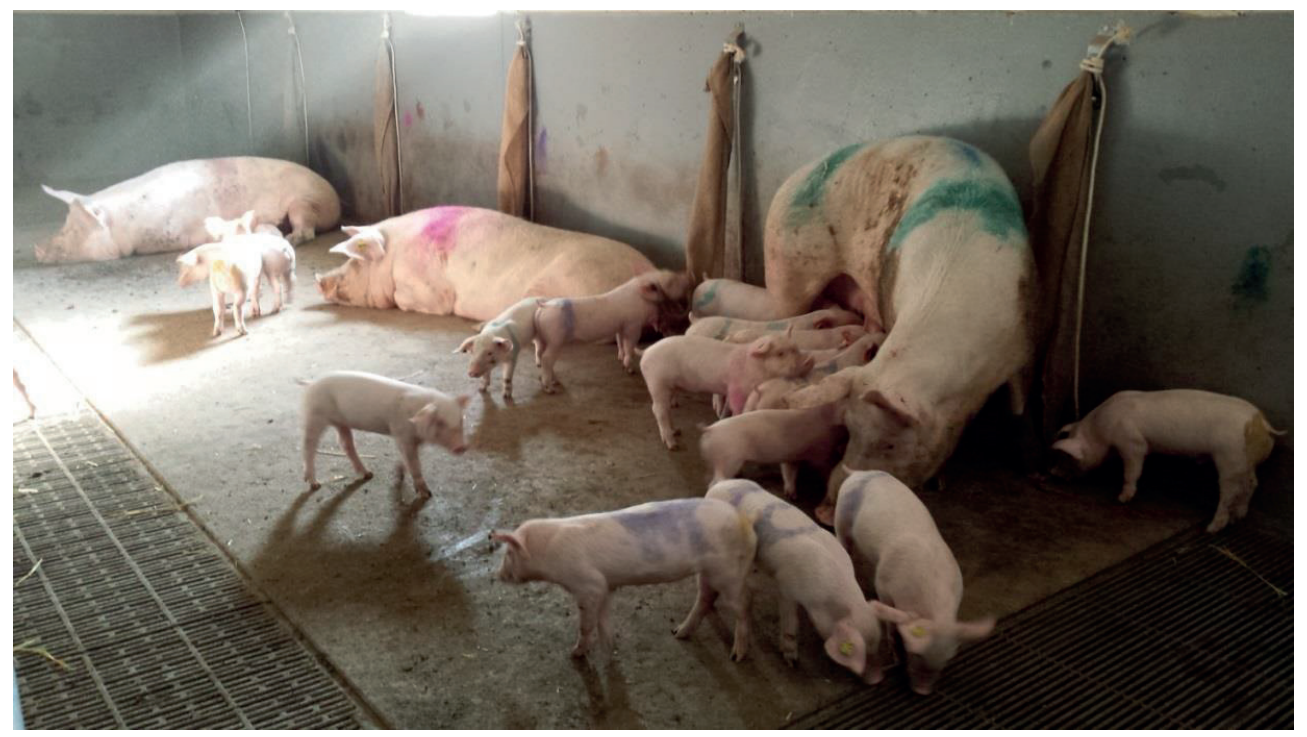

Figure 1.5. An impression of the multi-suckling system. 
MS system and subjected to gradual weaning during an extended lactation period of 9 weeks. Gradual weaning included intermittent-suckling in the fifth week (i.e. separation of sows and piglets for 10 hours daily), after which sows could voluntarily separate themselves from the piglets. Voluntary separation was possible by a connection between the MS units and an area that was inaccessible to the piglets. The piglets were prevented from entering this area by a barrier that the sows could step over. At 9 weeks of age, both groups were relocated to a finishing unit where they were housed under equal conditions. It was hypothesised that a more gradual weaning process would improve performance around transitions, potentially with beneficial effects on the longterm. Finally, Chapter 7 describes a discussion of the findings from all previously mentioned studies, to summarise and integrate the results. 


\subsection{References}

Andersen I.L., Vasdal G., and Pedersen L.J. 2014. Nest building and posture changes and activity budget of gilts housed in pens and crates. Applied Animal Behaviour Science 159: 29-33.

Barnett J.L., Hemsworth P.H., Cronin G.M., Jongman E.C., and Hutson G.D. 2001. A review of the welfare issues for sows and piglets in relation to housing. Australian Journal of Agricultural Research 52: 1-28.

Baxter E.M., Adeleye O.O., Jack M.C., Farish M., Ison S.H., and Edwards S.A. 2015. Achieving optimum performance in a loose-housed farrowing system for sows: The effects of space and temperature. Applied Animal Behaviour Science 169: 9-16.

Baxter E.M., Lawrence A.B., and Edwards S.A. 2012. Alternative farrowing accommodation: Welfare and economic aspects of existing farrowing and lactation systems for pigs. Animal 6: 96-117.

Bergeron R., Badnell-Waters A., Lambton S., and Mason G. 2006. Stereotypic oral behaviour in captive ungulates: foraging, diet and gastrointestinal function. Stereotypic Animal Behaviour: Fundamentals and Applications to Welfare 2: 19-41.

Bøe K. and Jensen P. 1995. Individual differences in suckling and solid food intake by piglets. Applied Animal Behaviour Science 42: 183-192.

Brambell F.W.R. 1965. Report of the Technical Committee to enquire into the welfare of animals kept under intensive livestock husbandry systems. Her Majesty's Stationery Office, London.

Bruininx E., Binnendijk G., Van der Peet-Schwering C., Schrama J., Den Hartog L., Everts H., and Beynen A. 2002. Effect of creep feed consumption on individual feed intake characteristics and performance of group-housed weanling pigs. Journal of animal science 80: 1413-1418.

Campbell J.M., Crenshaw J.D., and Polo J. 2013. The biological stress of early weaned piglets. Journal of animal science and biotechnology 4: 1 .

Castrén H., Algers B., de Passillé A.M., Rushen J., and Uvnäs-Moberg K. 1993. Preparturient variation in progesterone, prolactin, oxytocin and somatostatin 
in relation to nest building in sows. Applied Animal Behaviour Science 38: 91102.

CBS. 2014. Landbouw; biologisch en/of in omschakeling, gewassen, dieren, nationaal. Centraal bureau voor de statistiek, The Netherlands.

Columella L.J.M. 1941. On agriculture - with a recension of the text and an English translation by Harrison Boyd Ash Cambridge Harvard University Press.

Cronin G.M., Barnett J.L., Hodge F.M., Smith J.A., and McCallum T.H. 1991. The welfare of pigs in two farrowing/lactation environments: cortisol responses of sows. Applied Animal Behaviour Science 32: 117-127.

Dardaillon M. 1986. Seasonal variations in habitat selection and spatial distribution of wild boar (Sus scrofa) in the Camargue, Southern France. Behavioural Processes 13: 251-268.

Dexter N. 1999. The influence of pasture distribution, temperature and sex on homerange size of feral pigs in a semi-arid environment. Wildlife Research 26: 755762.

Dommerhold E.J. 1951. Het Varken. C. Misset.

Dybkjær L. 1992. The identification of behavioural indicators of 'stress' in early weaned piglets. Applied Animal Behaviour Science 35: 135-147.

Fishwick V.C. 1949. Pigs: Their Breeding, Feeding and Management. Crosby Lockwood \& Son, London.

Fraser D. 1978. Observations on the behavioural development of suckling and earlyweaned piglets during the first six weeks after birth. Animal Behaviour 26: 2230.

Fraser D., Mench J., and Millman S. 2001. Farm animals and their welfare in 2000.

Grimberg-Henrici C.G.E., Vermaak P., Elizabeth Bolhuis J., Nordquist R.E., and van der Staay F.J. 2016. Effects of environmental enrichment on cognitive performance of pigs in a spatial holeboard discrimination task. Animal Cognition 19: 271-283.

Gundlach H. 1968. Brutfürsorge, Brutpflege, Verhaltensontogenese und Tagesperiodik beim Europäischen Wildschwein (Sus scrofa L.)1. Zeitschrift für Tierpsychologie 25: 955-995. 
Gustafsson M., Jensen P., De Jonge F.H., Illmann G., and Spinka M. 1999. Maternal behaviour of domestic sows and crosses between domestic sows and wild boar. Applied Animal Behaviour Science 65: 29-42.

Harley S., Boyle L., O’Connell N., More S., Teixeira D., and Hanlon A. 2014. Docking the value of pigmeat? Prevalence and financial implications of welfare lesions in Irish slaughter pigs. Anim Welfare 23: 275-285.

Harrison R. 2013. Animal machines. Cabi.

Hartung J. 2013. A short history of livestock production. In: Aland A. and Banhazi T. (eds.) Livestock housing: Modern management to ensure optimal health and welfare of farm animals. p 21-34. Wageningen Academic Publishers, the Netherlands.

Hessel E.F., Reiners K., and Van Den Weghe H.F.A. 2006. Socializing piglets before weaning: Effects on behavior of lactating sows, pre- and postweaning behavior, and performance of piglets. Journal of Animal Science 84: 28472855.

Hillmann E., Von Hollen F., Bünger B., Todt D., and Schrader L. 2003. Farrowing conditions affect the reactions of piglets towards novel environment and social confrontation at weaning. Applied Animal Behaviour Science 81: 99-109.

Jarvis S., D'Eath R.B., Robson S.K., and Lawrence A.B. 2006. The effect of confinement during lactation on the hypothalamic-pituitary-adrenal axis and behaviour of primiparous sows. Physiology \& Behavior 87: 345-352.

Jensen P. 1986. Observations on the maternal behaviour of free-ranging domestic pigs. Applied Animal Behaviour Science 16: 131-142.

Jensen P. 1988. Maternal behaviour and mother-young interactions during lactation in free-ranging domestic pigs. Applied Animal Behaviour Science 20: 297-308.

Jensen P. 1989. Nest site choice and nest building of free-ranging domestic pigs due to farrow. Applied Animal Behaviour Science 22: 13-21.

Jensen P. 1995. The weaning process of free-ranging domestic pigs: within- and between-litter variations. Ethology 100: 14-25.

Jensen P., Florén K., and Hobroh B. 1987. Peri-parturient changes in behaviour in free-ranging domestic pigs. Applied Animal Behaviour Science 17: 69-76. 
Jensen P. and Recén B. 1989. When to wean - Observations from free-ranging domestic pigs. Applied Animal Behaviour Science 23: 49-60.

Jensen P. and Redbo I. 1987. Behaviour during nest leaving in free-ranging domestic pigs. Applied Animal Behaviour Science 18: 355-362.

Jensen P. and Stangel G. 1992. Behaviour of piglets during weaning in a seminatural enclosure. Applied Animal Behaviour Science 33: 227-238.

Jensen P., Stangel G., and Algers B. 1991. Nursing and suckling behaviour of seminaturally kept pigs during the first 10 days postpartum. Applied Animal Behaviour Science 31: 195-209.

Jensen P., Vestergaard K., and Algers B. 1993. Nestbuilding in free-ranging domestic sows. Applied Animal Behaviour Science 38: 245-255.

Kanaan V.T., Lay Jr D.C., Richert B.T., and Pajor E.A. 2012. Increasing the Frequency of Co-Mingling Piglets During the Lactation Period Alters the Development of Social Behavior Before and After Weaning. Journal of Applied Animal Welfare Science 15: 163-180.

King M., Kelly D., Morel P., and Pluske J. 2003. Aspects of intestinal immunity in the pig around weaning. In: Pluske J., Le Dividich J., and Verstegen M. (eds.) Weaning the Pig: Concepts and Consequences. p 219. Wageningen Academic Publishers, The Netherlands.

Lambertz C., Petig M., Elkmann A., and Gauly M. 2015. Confinement of sows for different periods during lactation: effects on behaviour and lesions of sows and performance of piglets. animal 9: 1373-1378.

Larson G. and Fuller D.Q. 2014. The evolution of animal domestication. Annual Review of Ecology, Evolution, and Systematics 45: 115-136.

Legault C. 1985. Selection of breeds, strains and individual pigs for prolificacy. Journal of reproduction and fertility. Supplement 33: 151-166.

Lidfors L., Berg C., and Algers B. 2005. Integration of Natural Behavior in Housing Systems. AMBIO: A Journal of the Human Environment 34: 325-330.

McGlone J.J. and Curtis S.E. 1985. Behavior and performance of weanling pigs in pens equipped with hide areas. Journal of Animal Science 60: 20-24.

Meynhardt H. 1980. Schwarzwild-Report: Vier Jahre unter Wildschweinen. Verlag J. Neumann-Neudamm, Berlin, Germany. 
Miller H. and Slade R. 2003. Digestive physiology of the weaned pig. In: Pluske J., Le Dividich J., and Verstegen M. (eds.) Weaning the pig: concepts and consequences. Wageningen Academic Publishers, The Netherlands.

Morgan C.A., Lawrence A.B., Chirnside J., and Deans L.A. 2001. Can information about solid food be transmitted from one piglet to another? Animal Science 73: $471-478$.

Newberry R.C. and Wood-Gush D.G.M. 1985. The Suckling Behaviour of Domestic Pigs in a Semi-Natural Environment. Behaviour 95: 11-25.

Oostindjer M., Bolhuis J.E., Mendl M., Held S., van den Brand H., and Kemp B. 2011. Learning how to eat like a pig: Effectiveness of mechanisms for vertical social learning in piglets. Animal Behaviour 82: 503-511.

Oostindjer M., Kemp B., van den Brand H., and Bolhuis J.E. 2014. Facilitating 'learning from mom how to eat like a pig'to improve welfare of piglets around weaning. Applied Animal Behaviour Science 160: 19-30.

Pajor E.A., Fraser D., and Kramer D.L. 1991. Consumption of solid food by suckling pigs: individual variation and relation to weight gain. Applied Animal Behaviour Science 32: 139-155.

Petersen V., Recén B., and Vestergaard K. 1990. Behaviour of sows and piglets during farrowing under free-range conditions. Applied Animal Behaviour Science 26: 169-179.

Podgórski T., Lusseau D., Scandura M., Sönnichsen L., and Jędrzejewska B. 2014. Long-lasting, kin-directed female interactions in a spatially structured wild boar social network. PloS one 9: e99875.

Rushen J. 2008. Farm animal welfare since the Brambell report. Applied Animal Behaviour Science 113: 277-278.

Russo L., Massei G., and Genov P. 1997. Daily home range and activity of wild boar in a Mediterranean area free from hunting. Ethology Ecology \& Evolution 9: 287-294.

Saunders G. and McLeod S. 1999. Predicting home range size from the body mass or population densities of feral pigs, Sus scrofa (Artiodactyla: Suidae). Australian Journal of Ecology 24: 538-543. 
Schley L. and Roper T.J. 2003. Diet of wild boar Sus scrofa in Western Europe, with particular reference to consumption of agricultural crops. Mammal Review 33: 43-56.

Settis S. and Bonamici M. 1985. The land of the Etruscans: from prehistory to the Middle Ages. Scala Books.

Singer F.J., Otto D.K., Tipton A.R., and Hable C.P. 1981. Home ranges, movements, and habitat use of European wild boar in Tennessee. The Journal of Wildlife Management: 343-353.

Sneddon I., Beattie V., Dunne L., and Neil W. 2000. The effect of environmental enrichment on learning in pigs. Animal Welfare 9: 373-383.

Špinka M., Illmann G., de Jonge F., Andersson M., Schuurman T., and Jensen P. 2000. Dimensions of maternal behaviour characteristics in domestic and wild $\times$ domestic crossbred sows. Applied Animal Behaviour Science 70: 99114.

Stangel G. and Jensen P. 1991. Behaviour of semi-naturally kept sows and piglets (except suckling) during 10 days postpartum. Applied Animal Behaviour Science 31: 211-227.

Stolba A. and Wood-Gush D. 1989. The behaviour of pigs in a semi-natural environment. Animal production 48: 419-425.

Studnitz M., Jensen M.B., and Pedersen L.J. 2007. Why do pigs root and in what will they root?: A review on the exploratory behaviour of pigs in relation to environmental enrichment. Applied Animal Behaviour Science 107: 183-197.

Thornton P.K. 2010. Livestock production: recent trends, future prospects. Philosophical Transactions of the Royal Society B: Biological Sciences 365: 2853-2867.

Veissier I., Butterworth A., Bock B., and Roe E. 2008. European approaches to ensure good animal welfare. Applied Animal Behaviour Science 113: 279-297.

Vente-Spreeuwenberg M. and Beynen A. 2003. Diet-mediated modulation of small intestinal integrity in weaned piglets. In: Pluske J., Le Dividich J., and Verstegen M. (eds.) Weaning the pig: concepts and consequences. p 145-198.

Weary D.M., Jasper J., and Hötzel M.J. 2008. Understanding weaning distress. Applied Animal Behaviour Science 110: 24-41. 
Weng R.C., Edwards S.A., and English P.R. 1998. Behaviour, social interactions and lesion scores of group-housed sows in relation to floor space allowance. Applied Animal Behaviour Science 59: 307-316.

Wischner D., Kemper N., and Krieter J. 2009. Nest-building behaviour in sows and consequences for pig husbandry. Livestock Science 124: 1-8.

Yun J., Swan K.-M., Farmer C., Oliviero C., Peltoniemi O., and Valros A. 2014a. Prepartum nest-building has an impact on postpartum nursing performance and maternal behaviour in early lactating sows. Applied Animal Behaviour Science 160: 31-37.

Yun J., Swan K.-M., Oliviero C., Peltoniemi O., and Valros A. 2015. Effects of prepartum housing environment on abnormal behaviour, the farrowing process, and interactions with circulating oxytocin in sows. Applied Animal Behaviour Science 162: 20-25.

Yun J., Swan K.M., Vienola K., Kim Y.Y., Oliviero C., Peltoniemi O.A.T., and Valros A. 2014b. Farrowing environment has an impact on sow metabolic status and piglet colostrum intake in early lactation. Livestock Science 163: 120-125.

Yun J. and Valros A. 2015. Benefits of prepartum nest-building behaviour on parturition and lactation in sows-a review. Asian-Australasian journal of animal sciences 28: 1519 . 



\section{CHAPTER 2}

\section{A review of sow and piglet behaviour and performance in group housing systems for lactating sows}

S.E. van Nieuwamerongen ${ }^{1 *}$, J.E. Bolhuis ${ }^{1}$, C.M.C. van der PeetSchwering $^{2}$, N.M. Soede ${ }^{1}$

${ }^{1}$ Adaptation Physiology Group, Department of Animal Sciences, Wageningen University, PO Box 338, 6700 AH Wageningen, The Netherlands;

${ }^{2}$ Wageningen UR Livestock Research, PO Box 65, 8200 AB, Lelystad, The Netherlands.

*Corresponding author: S.E. van Nieuwamerongen at Sofie.vannieuwamerongen@wur.nl. 


\subsection{Abstract}

Commercial use of group housing systems for lactating sows is limited, but the recent transition to group housing during gestation in the EU may result in a renewed interest in such systems. Therefore, this review aims to identify key factors that may contribute to the success or failure of group housing of lactating sows in comparison with individual housing, by describing the variety in group housing systems and discussing animal behaviour and performance compared with individual housing. Group housing systems can be divided in multi-suckling (MS) systems, in which sows are grouped with their litters, and get-away (GA) systems, which include a separate communal area accessible to sows only. These systems differ in many aspects regarding management and layout but, compared with individual housing, generally provide more environmental complexity, more freedom of movement for the sows, and more freedom to express behaviours related to, for example, maternal care and social interactions. Group housing poses several risks, such as disrupted nursing and an increased level of crushing during the MS phase, and in GA systems there is a risk for early cessation of nursing. On the other hand, pre-weaning mingling of litters clearly benefits piglet social development and may improve adaptation to the post-weaning situation. In addition, group housed sows may show lactational ovulation, which provides opportunities for insemination during an extended lactation period, which benefits the piglets. Gradual transitions in social and physical environment around gestation, farrowing, grouping, and weaning seem to be key success factors for group housing systems during lactation. In addition, selection of suitable sows and quality of stockmanship seem important.

Keywords: multi-suckling, cross-suckling, social development, lactational ovulation, pig. 


\subsection{Implications}

The recent ban on individual housing of gestating sows in the EU may result in a renewed interest in group housing systems for lactating sows. Commercial use of such systems is, however, limited and this review attempts to identify key factors that may contribute to the success or failure of group housing of lactating sows in comparison with individual housing. By providing more insight in these factors, this review may be useful in developing and optimising feasible group housing systems for lactating sows.

\subsection{Introduction}

Public demand for more welfare-friendly pig husbandry systems has resulted in a ban on individual housing of sows for the major part of gestation in the EU (Council Directive 2001/88/EC). During farrowing and lactation, however, most sows are still individually confined in crates. Several studies have investigated alternative farrowing systems over the years, such as group housing of sows during lactation (e.g. Wechsler, 1996). Such systems potentially provide a better transition from group housing during gestation and may facilitate the expression of natural behaviour. Wild boar and feral pigs live in family groups of several females with offspring. Under semi-natural conditions, peri-parturient free-ranging sows separate from the group to investigate nest sites 15 to 24 hours before farrowing. Sows build a nest by digging a shallow oval hole and covering it with plant material. When the litter is born, the sow and her piglets initially remain in proximity of the nest. Around 2 days post-partum (p.p.), the sow starts leaving the nest for short foraging trips and 1 day later the piglets gradually follow (Jensen, 1986). The sow and her piglets abandon the nest and return to the group on an average of 9 to 10 days p.p. (Jensen, 1986; Jensen and Redbo, 1987) and the litter is gradually integrated in the family group (Jensen, 1986). From early lactation (weeks 1 to 4 p.p.), sows start to decrease nursing frequency and actively terminate an increasing proportion of nursings (Jensen, 1988; Jensen and Recén, 1989), whereas piglets gradually start to sample and ingest solid feed (Meynhardt, 1980). In nature, weaning is thus a gradual process, which is completed between 14 and 22 weeks p.p. (Jensen, 1986, 1988; Jensen and Recén, 1989). 
Group housing of sows during (part of) lactation -especially in combination with grouping of litters- more closely resembles the natural situation and may benefit welfare of sows and piglets by providing more freedom of movement, an improved control of nursing behaviour by the sows, and more possibilities for exploration and social interaction. The latter may facilitate social learning and development of the piglets (Kutzer et al., 2009; Oostindjer et al., 2011), and may consequently facilitate adaptation to weaning. Despite these possible benefits of group housing during lactation, commercial use of such systems is limited. The recent transition to group housing during gestation in the EU may, however, result in a renewed interest in group housing systems for lactating sows. Recent papers have evaluated farrowing systems for pigs (Johnson and Marchant-Forde, 2009; Baxter et al., 2011, 2012), but a review fully focussing on group housing systems is lacking. Therefore, this review aims to identify key factors that may contribute to the success or failure of group housing of lactating sows in comparison with individual housing by (i) describing the variety in sow group housing systems during lactation and (ii) discussing animal behaviour and performance in such systems compared with individual housing. By providing more insight in these key factors, this review may be useful in developing and optimising feasible group housing systems for lactating sows.

\subsection{Variation in sow group housing systems during lactation}

The majority of reviewed papers concern relatively small-scale experimental studies focussed on behaviour and production performance, although also some larger scale studies on commercial farms in Sweden are included. The group housing systems for sows during (part of) lactation vary in many aspects (Table 2.1). Roughly, a distinction can be made between systems which include a communal area accessible to sows only during the whole lactation period (get-away (GA) systems) and systems with group housing of sows together with their litters (multi-suckling (MS) systems). In some MS systems, GA housing precedes MS housing, but there are many more options to combine farrowing and lactation housing (also see Johnson and MarchantForde, 2009). Figure 2.1 presents the most common transitions in the systems studied. 
Table 2.1. Variation in 42 reviewed studies on sow group housing during (part of) lactation.

\begin{tabular}{|c|c|c|}
\hline & No. of studies $^{1}$ & Median (range) $^{2}$ \\
\hline Group housing system ${ }^{3}$ & 42 & - \\
\hline Get-away system ${ }^{4}$ & 7 & - \\
\hline Multi-suckling system & 35 & - \\
\hline Number of sows per study & 38 & $40(10$ to 840$)$ \\
\hline Housing during gestation & 32 & - \\
\hline Group housing & 29 & - \\
\hline Partly grouped, partly individual & 3 & - \\
\hline Farrowing housing ${ }^{5}$ & 41 & - \\
\hline Farrowing crate ${ }^{6}$ & 12 & - \\
\hline Loose housing & 31 & - \\
\hline Sow group size & 42 & $6\left(3\right.$ to $\left.30^{7}\right)$ \\
\hline \multicolumn{3}{|l|}{ Communal area } \\
\hline $\mathrm{m}^{2}$ per sow & 38 & $8(2.2$ to 4000$)$ \\
\hline Floor type (indoor) & 16 & - \\
\hline Concrete & 10 & - \\
\hline (Partly) slatted & 6 & - \\
\hline Bedding & 29 & - \\
\hline (Partly) straw bedded & 24 & - \\
\hline None & 5 & - \\
\hline Timing of grouping sows (days p.p.) ${ }^{8}$ & 37 & $10(-21$ to 21$)$ \\
\hline Timing of grouping piglets (days p.p.) ${ }^{9}$ & 35 & $11(0$ to 35$)$ \\
\hline Piglet feed & 32 & - \\
\hline In creep area & 22 & - \\
\hline (Also) access to sow feeder ${ }^{10}$ & 5 & - \\
\hline Other & 5 & - \\
\hline Age at weaning (days p.p.) $)^{11}$ & 38 & 32 (19 to 56$)$ \\
\hline \multicolumn{3}{|l|}{ Housing of control group ${ }^{12}$} \\
\hline Farrowing crate & $11^{13}$ & - \\
\hline Loose housing & $14^{14}$ & - \\
\hline No control group & 18 & - \\
\hline
\end{tabular}

p.p. = post-partum

${ }^{1}$ Not all characteristics were clearly reported in each study and, therefore, the number of studies does not always equal 42 .

${ }^{2}$ If ranges were given in papers (e.g. regarding sow group size), the average was used in calculations.

${ }^{3}$ Of which 10 (partly) outdoor.

${ }^{4}$ Of which 2 studies with an additional separate litter mingling area.

${ }^{5}$ Of which 2 studies with 2 different MS systems (preceded by either farrowing in crates or loose farrowing).

${ }^{6}$ Of which 2 studies in which sows were loose-housed several days after farrowing.

${ }^{7}$ Group size of 30: concerns a system in which lactating sows were integrated with dry and gestating sows.

${ }^{8}$ Of which 5 studies with sows grouped pre-farrowing, but locked in own pen around farrowing.

${ }^{9}$ In MS housing only.

${ }^{10}$ Only considered when specifically stated.

${ }^{11}$ In 1 study voluntary weaning was allowed.

${ }^{12}$ Of which 1 study with both crated and loose-housed sows as control.

${ }^{13}$ Of which 1 study with tethered sows and 1 study in which sows were loose-housed after day 20 p.p.

${ }^{14}$ Of which 6 studies in which (part of) the sows were crated around farrowing. 


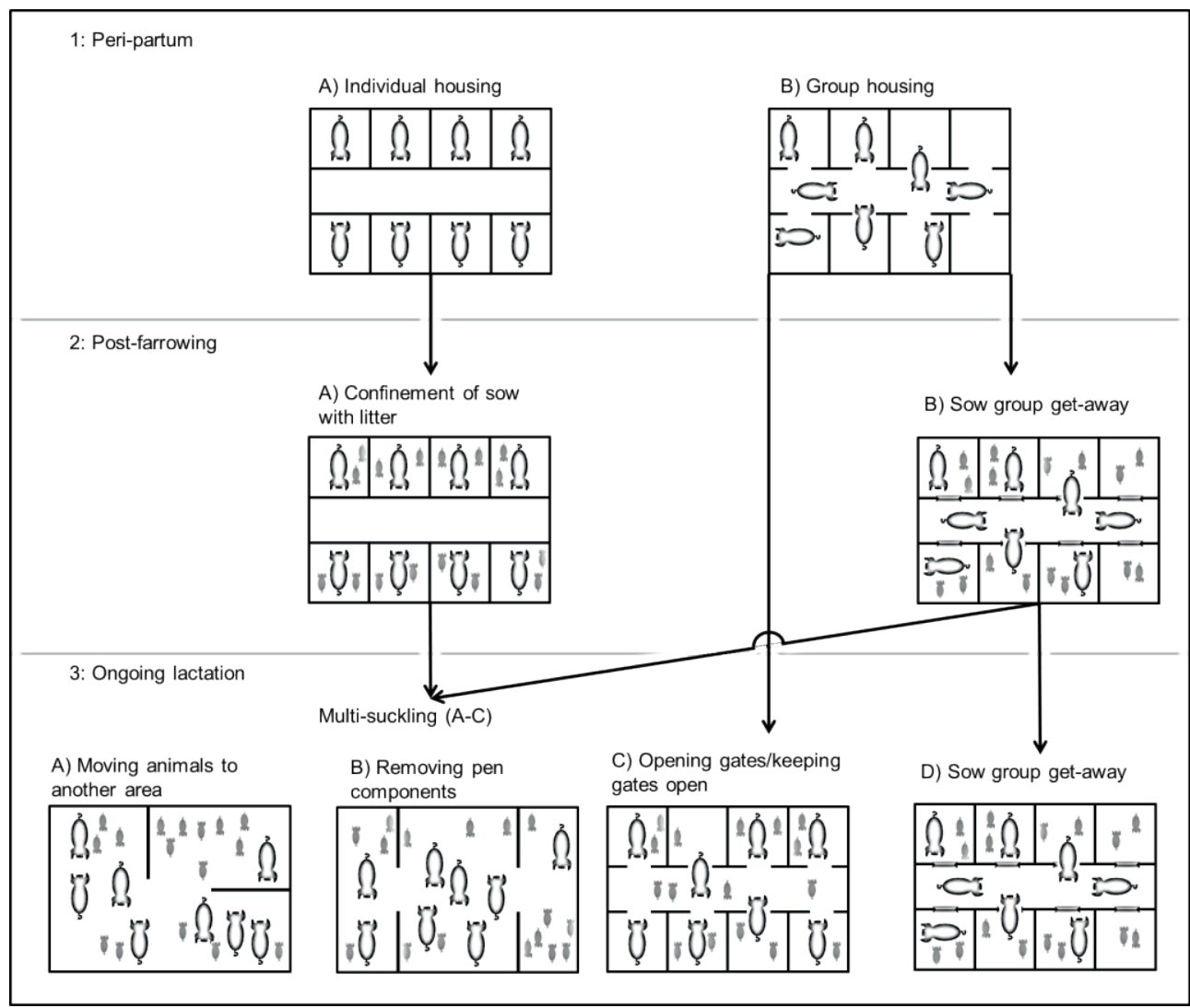

Figure 2.1. Variation in sow group housing systems during (part of) lactation. During the peripartum period (1), sows can be housed individually (1A) or group-wise (1B). When group housed during the peri-partum period, sows can often choose their own farrowing accommodation. Sow access to farrowing crates or pens is either unrestricted (1B and $3 \mathrm{C}$ ) or regulated electronically to avoid multiple sows from entering the same pen and prevent the access of sows to other litters. After farrowing ( 2 and 3 ), sows can be kept with their litter for a certain period (2A) before grouping all animals by moving animals to another area (3A), removing pen components $(3 \mathrm{~B})$, or opening gates $(3 \mathrm{C})$. In case both sows and litters are grouped together, this can occur either simultaneously or sequentially. The layout of a free access system remains the same and all animals can access all areas (3C: keeping gates open). Alternatively, in a sow group get-away system (2B), litters can be kept separated (3D) or mixed after a certain period using previously described methods $(3 \mathrm{~A} / 3 \mathrm{~B} / 3 \mathrm{C})$. 


\subsection{Sow and piglet behaviour and performance}

\subsubsection{Nursing and suckling behaviour}

\section{Nursing frequency and success}

Nursing behaviour is the result of interaction between the sow and her litter. Under semi-natural conditions, the nursing frequency of domestic pigs and wild boar generally decreases over the course of lactation (Figure 2.2), with a decreasing proportion of nursings initiated by the sow and an increasing proportion of nursings terminated by the sow (Horrell, 1997).

In GA systems -in which sows can completely evade nursing requests of their piglets- it may be expected that nursing behaviour is more regulated (i.e. restricted) by the sows than in a natural environment or in individual housing. Correspondingly, Weary et al. (2002) reported that the frequency of successful nursings (i.e. with milk let-down) was lower in a GA system than in individual loose housing. In addition, the nursing frequency in GA systems seems to decline more rapidly compared with semi-natural conditions and farrowing crates (Figure 2.2). At 4 weeks p.p. the proportion of nursings terminated by the sow was about $95 \%$ in a GA system (Bøe, 1993) and about $62 \%$ in individual housing (Valros et al., 2002). This possibly reflects a decreased nursing motivation in GA systems, as by terminating a nursing bout the sow prevents further udder massage by the piglets.

As MS systems are usually designed to better enable expression of natural behaviour, it may be expected that the nursing frequency in MS systems is similar to that under natural conditions. Compared with individual housing, the frequency of successful nursings seems quite similar in MS systems (Figure 2.2), which is supported by Bohnenkamp et al. (2013a). Within MS systems, the frequency of successful nursings is affected by pre-grouping housing: sows that farrowed in a group GA system nursed more often in the MS phase compared with sows that farrowed in a crate (1.41/hour vs. 1.34 /hour, with a similar proportion of successful nursings) (Dybkjær et al., 2001). In addition, other studies suggest that a relatively drastic change in physical and social environment may result in decreased nursing success, possibly because of stress (Rushen et al., 1995). The percentage of nursings with milk let-down dropped; from $72 \%-98 \%$ to $29 \%-57 \%$ after pigs were relocated from farrowing crates to a 


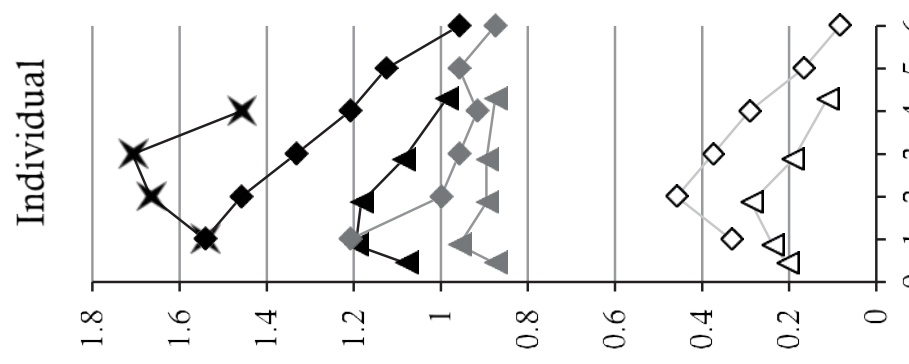

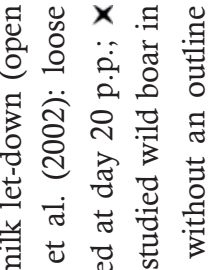

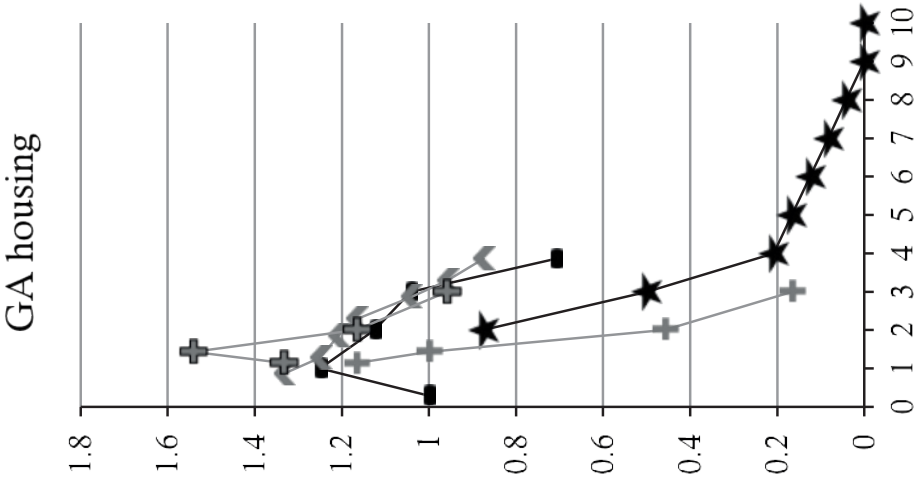

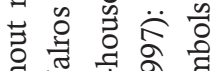

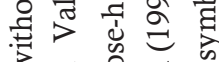

$\infty<0$ 宓

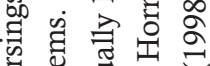

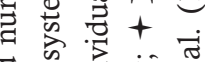

वี की

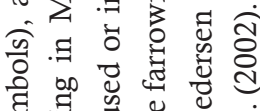

क्ञ ज्ञ

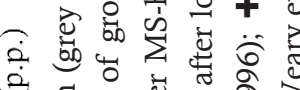

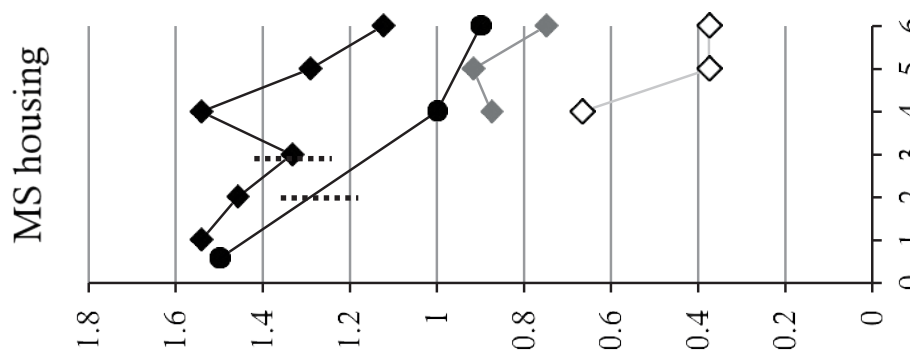

₹

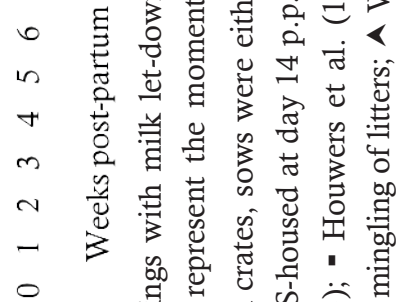

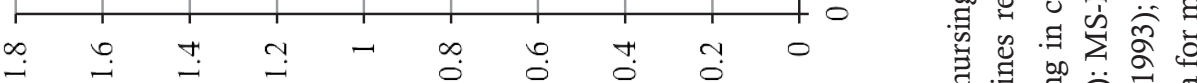

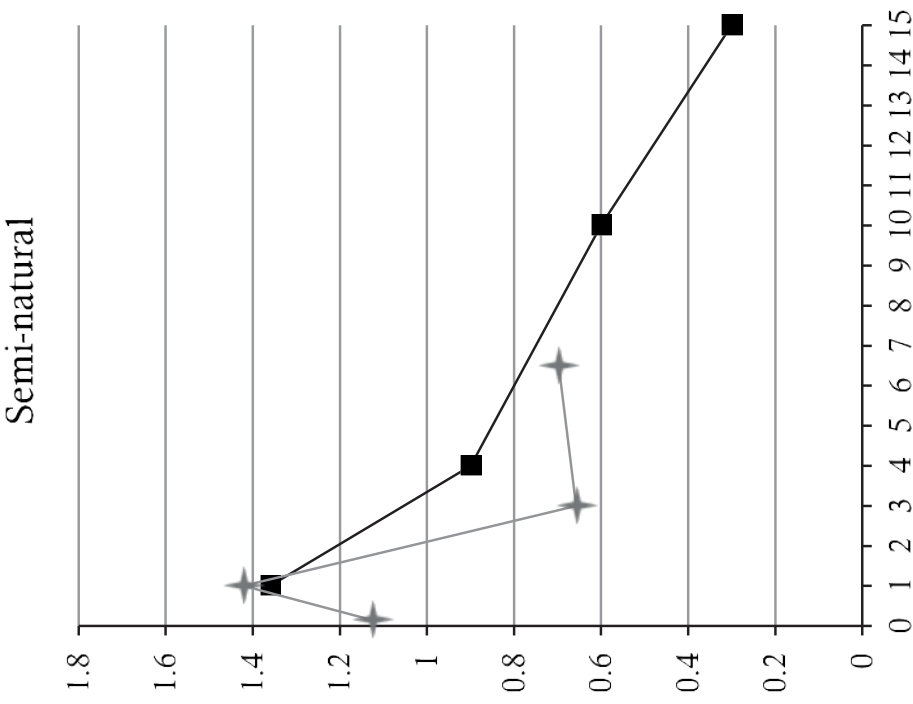

计

帘

栗

के 巡芯芯芯

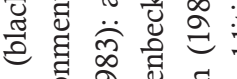

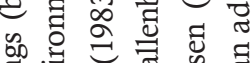

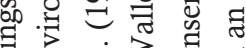

क्षै तं है

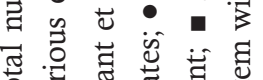

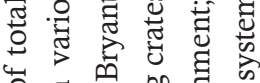

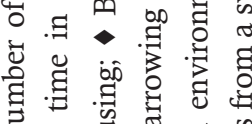

乙 పे

ํ.

응

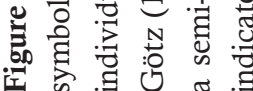

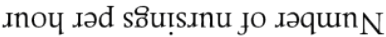


new MS environment (Bryant et al., 1983; Wattanakul et al., 1997; Wattanakul et al., 1998), whereas nursing success remained rather constant in the same period in farrowing crates (Wattanakul et al., 1997), even after relocation from farrowing crates to loose individual housing (Bryant et al., 1983). Further, in an MS system from birth, nursing success was higher than in farrowing crates (96.7\% vs. 93.2\%, SED = 1.3, Arey and Sancha 1996).

In conclusion, in GA systems, sows have more freedom to regulate nursing behaviour compared with a natural environment and individual housing. The nursing behaviour in MS systems seems to be affected by the transitions in terms of physical and social environment within these systems.

\section{Cross-suckling}

Milk ingestion from a sow other than the own mother (i.e. cross-suckling or allo-suckling) may occur in a natural environment (Meynhardt, 1980). Sows may nurse non-offspring piglets because this can provide inclusive fitness benefits (Roulin, 2002), given that groups of pigs in a natural environment are often composed of related individuals (Meynhardt, 1980). Moreover, sows may nurse non-offspring to remove leftover milk and reduce pressure in the udder (Roulin, 2002) or cross-suckling may occur inadvertently because the sow does not notice non-offspring piglets.

The effects of cross-suckling on piglet performance are not straightforward. It has been reported that birth and weaning weights were similar for crosssucklers and non-cross-sucklers, both in MS housing (Olsen et al., 1998) and in farrowing crates in which litters could mingle (Illmann et al., 2007). Dybkjær et al. (2001), however, found a negative correlation between cross-suckling frequency and daily growth $(r=-0.81)$, and others reported a lower preweaning weight gain for cross-sucklers compared with non-cross-sucklers (Goetz and Troxler 1995; Wülbers-Mindermann 1992, cited in BurgwalKonertz 1996). On the one hand, cross-suckling may provide benefits for piglets that have a low milk intake from their own mother (Olsen et al., 1998) and even lead to greater piglet uniformity when cross-suckling levels out differences in litter size (Algers, 1991). On the other hand, competition at the udder may be increased (Pedersen et al., 1998), which may be detrimental for the weakest piglets (Algers, 1991), cause stress owing to a lack of a stable milk source (Burgwal-Konertz, 1996), and result in injuries to the head and carpal 
joints (Goetz and Troxler, 1995). In addition, sows may be distressed by crosssuckling and cross-massaging of piglets (Bohnenkamp et al., 2013a), and sows can be more aggressive towards non-offspring (Olsen et al., 1998). These potential negative consequences make cross-suckling an issue that needs further attention in MS systems (Olsen et al., 1998).

In MS systems, the percentage of cross-sucklers relative to the total number of piglets varied from 11 to 39\% (Goetz and Troxler, 1995; Wechsler, 1996; Olsen et al., 1998; Maletínská and Špinka, 2001), the percentage of cross-sucklers per nursing ranged from $10 \%$ to $65 \%$ (Wattanakul et al., 1997; Wattanakul et al., 1998), and the number of nursings in which cross-suckling occurred varied from 29\% to $62 \%$ (Bryant and Rowlinson, 1984; Maletínská and Špinka, 2001). Cross-sucklers display different strategies regarding the number of other sows they target and how often they cross-suckle, that is, permanently or nonpermanently (Goetz and Troxler, 1995; Olsen et al., 1998; Maletínská and Špinka, 2001). In literature, several factors contributing to the development and attraction of cross-sucklers have been identified.

\section{Animal-related factors}

First, milk yield of the sow may affect the development of cross-suckling. Olsen et al. (1998) noted that litters with a higher weight gain before grouping produced fewer cross-sucklers in the MS phase than litters with lower weight gains before grouping and cross-sucklers most often switched to a sow with a higher milk yield (estimated by pre-grouping litter gain of the sow). Large litters produced more cross-sucklers (Wülbers-Mindermann, 1992 cited in Burgwal-Konertz 1996), particularly more permanent cross-sucklers (Maletínská and Špinka, 2001), which is likely mediated by the number of available functional teats. Olsen et al. (1998) found no litter size effect, possibly because of smaller variation in litter size. Wechsler (1996) noted that all crosssucklers switched to sows with the least number of piglets in the group. Goetz and Troxler (1995) found that non-permanent cross-sucklers targeted sows with smaller litter sizes than their own mother, but permanent cross-sucklers had no preference regarding litter size. Possibly, equalising litter sizes and adjusting the number of suckling piglets per sow to the number of functional teats limits cross-suckling. Variable effects of sow parity on the occurrence of crosssuckling were reported (Olsen et al., 1998; Maletínská and Špinka, 2001), 
possibly because parity may be confounded with milk yield or litter size. Finally, sows in MS housing may synchronise their nursing behaviour, which reduces the presence of non-offspring piglets at milk let-down (Maletínská and Špinka, 2001). Nursing synchronisation (i.e. nursing within 2 to 3 minute intervals from another nursing) was higher in MS housing than in individual housing (Wattanakul et al., 1997; Šilerová et al., 2006), specifically in the days after grouping (Bryant et al., 1983; Wattanakul et al., 1997).

\section{Management-related factors}

In most studies on MS housing, sows and their piglets were kept together for at least 1 week before grouping the litters. In the first week p.p. sow-piglet recognition and bonding occurs (Horrell and Hodgson, 1992) and it might be expected that cross-suckling is increased if litters are grouped before 1 week of age. Bohnenkamp et al. (2013a), however, grouped litters at 5 days p.p. and found a low level of cross-suckling. This might be because sow-piglet bonding had already sufficiently occurred or because the sows were still able to nurse in their own crate. Within their study, more non-offspring piglets were present per nursing bout in the open MS area compared with the individual crates. Furthermore, although MS-housed sows were more aggressive towards crossfostered piglets, these piglets were not more likely to become cross-sucklers (Olsen et al., 1998). Wechsler (1996) and Maletínská and Špinka (2001) found that cross-suckling was affected by the variation in litter age within a group $(\mathrm{r}=$ 0.50 , observed between 19 to 32 days p.p.). Although older piglets crosssuckled more often, they did not miss more nursings at their mother (Maletínská and Špinka, 2001). Finally, previous housing conditions can affect the occurrence of cross-suckling. Dybkjær et al. (2001) found a lower frequency of cross-suckling in the MS phase starting 11 days p.p. when sows had farrowed in a GA system compared with when sows had farrowed in crates (7.4 \pm 2.0 vs. $25.1 \pm 9.1$ occurrences/sow per 24 hours on day 24 p.p.). Similarly, Wattanakul et al. (1998) found that the percentage of cross-suckling piglets per nursing was about 2.4 to 5.4 times higher when litters came from farrowing crates and were simultaneously relocated and mixed in a new MS environment 2 weeks p.p., compared with litters that came from farrowing crates, were relocated to loose individual housing 7 days p.p., and gained access to an MS area connected to their pens a week thereafter. This effect on cross-suckling may have resulted from a difference in familiarity with the 
environment, which is also suggested by Wattanakul et al. (1997). Therefore, providing a smooth transition to group housing may limit cross-suckling and possibly reduces the decrease in nursing success, which may be observed after grouping.

Hence, although cross-suckling has some potentially positive aspects, it can be seen as a problem if it negatively affects piglet performance and causes restlessness and disrupted nursing. The level of milk intake at the mother, differences in litter age within a group, and the transition to MS housing seem to affect the level of cross-suckling.

\section{Cessation of nursing}

In sow group housing, complete cessation of nursing before the designated moment of weaning may occur. The consequences for piglet behaviour and performance may depend on the piglets' age (Worobec et al., 1999) and their ability to compensate with increased solid food intake. The risk of nursing cessation before the intended moment of weaning may be higher in GA systems than in MS systems because GA-housed sows can evade piglets' nursing requests.

In GA systems, weaning before 5 weeks p.p. occurred in $56 \%(9 / 16)$ of sows (Bøe, 1993) and weaning before 3 weeks p.p. occurred in $8.3 \%(1 / 12)$ of sows (Pedersen et al., 1998). More sows ( $9 / 12$ vs. $1 / 12)$ weaned their piglets before 3 weeks of age in a GA system in which litters could access multiple pens, compared with a GA system with separated litters. Restlessness was increased because of higher competition at the udder and milk intake was negatively affected (Pedersen et al., 1998). In MS housing, nursing cessation indeed seems to occur at a lower level. At weaning between 5 and 6 weeks p.p., Hultén et al. $(1995 \mathrm{a}, 1995 \mathrm{~b})$ reported complete udder atrophy in, respectively, 5.0\% and $6.6 \%$ of the multiparous sows, whereas Hultén et al. (2006) did not note termination of milk production before weaning at 7 weeks of age. Udder atrophy before weaning never occurred in individual housing and in first parity MS-housed sows (Hultén et al., 1995a; Hultén et al., 1995b). Bohnenkamp et al. (2013a), however, reported that primiparous sows were more disturbed around nursing and reduced their nursing frequency sooner than multiparous sows did. 
Summarising, the risk of cessation of nursing before the intended moment of weaning may be higher in GA systems than in MS systems and may be linked to, for example, disturbances around nursing and sow parity.

\subsubsection{Piglet mortality}

Piglet mortality is a major concern in pig husbandry (Baxter et al., 2012). The design of the farrowing pen can greatly affect piglet losses, which mostly occur within the first few days after birth (Marchant et al., 2000). Farrowing pen design is, however, highly variable in the studies reviewed and therefore total pre-weaning mortality in individual housing and group housing will not be compared. Instead, risk factors characteristic to group housing will be addressed. For instance, providing sows freedom to choose a preferred site of farrowing may increase the risk of piglet mortality. Burgwal-Konertz (1996) observed that $12.5 \%(6 / 48)$ of sows farrowed dispersed in the communal area or in multiple pens, and Houwers et al. (1996) reported that only $76 \%$ of sows voluntarily farrowed in an empty pen. Scattered farrowing or farrowing in an occupied pen may increase the risk of crushing, malnourishment, and hypothermia (Burgwal-Konertz, 1996). Furthermore, in an on-farm study on 305 sows, Hultén et al. (1997) reported higher mortality in the MS phase from 2 to 3 until 5 to 6 weeks p.p. compared with individual housing (6.5\% vs. $1.4 \%$ ), whereas piglet losses before grouping were similar (9.6\%). In smallerscale studies, however, no difference in mortality was found during the MS phase (Rantzer et al., 1997; Wattanakul et al., 1997). Hultén et al. (1997) identified a large litter size at grouping as a risk factor for mortality during the MS phase. In addition, multiparous sows had higher levels of piglet losses after grouping (parity 2 to $4: 5.3 \%$; parity $\geq 5: 8.6 \%$ ) compared with loose individual housing (parity 2 to $4: 1.1 \%$; parity $\geq 5: 1.2 \%$ ). Among first parity sows, mortality after grouping did not differ $(5.6 \%$ vs. $2.4 \%$ in loose individual housing). Furthermore, a transition from farrowing crates to a new MS area 11 days p.p. tended to result in higher mortality after grouping than a transition from a group GA system to MS housing (6.5\% vs. 4.4\%, Dybkjær et al., 2001). In general, piglet mortality may be reduced with increasing experience of the stockpersons with group housing. Wechsler (1996) and Li et al. (2010) reported reduced piglet mortality in an MS system over the years, partly owing to improved skills of stockpersons, related to better observation and handling of animals, and a better approach to occurring problems (Li et al., 2010). 
Crushing or trauma caused $41 \%$ to $70 \%$ of all deaths in the MS phase starting from 1 to 2 weeks p.p. (Rantzer et al., 1997; Dybkjær et al., 2001; Dybkjær et al., 2003). In individual loose housing, $27.2 \%$ of mortality was due to crushing from 1 week p.p. (Rantzer et al., 1997). Several studies found an increased level of crushing during the MS phase if litters were housed with crated sows before grouping of litters, compared with continuous housing in farrowing crates (Marchant et al., 2000; Wattanakul et al., 1997), which may be explained by sudden exposure to 'dangerous' lying behaviour of the sows (Marchant et al., 2000). In an MS system from birth, it was indicated that MShoused sows were more careful in their lying behaviour, as they showed a higher and more consistent standing-up response to playbacks of piglet vocalisations compared with sows housed in crates (Arey and Sancha, 1996). In addition, in MS housing preceded by individual housing, a higher level of crushing may result from an increased activity level of sows (Wattanakul et al., 1997).

In conclusion, piglet mortality may be higher in the MS phase compared with individual housing. Specifically, the level of crushing may be increased if MS housing is preceded by housing in farrowing crates because of exposure to 'dangerous' lying behaviour of the sows. Piglet mortality may improve with increasing experience of stockpersons with group housing systems.

\subsubsection{Piglet (social) development}

In contrast to individual housing, MS housing enables social contact between multiple sows and litters, and provides a larger and more complex environment. This more closely resembles the natural situation and is expected to benefit piglet development. Separating the first acquaintance with nonlittermates from the moment of weaning reduces the number of concurrent stressors weaned piglets have to deal with. Moreover, when multiple litters are grouped during lactation in MS housing, post-weaning mixing may be unnecessary altogether.

Mixing litters before 2 weeks of age generally results in little aggression and few skin lesions (Wattanakul et al., 1997; Kutzer et al., 2009), which is in contrast with the vigorous fighting and skin damage that is reported for pigs mixed at weaning after 4 weeks p.p. (Melotti et al., 2011). Compared with piglets that were first mixed at weaning at an age of 3 to 4 weeks, piglets that 
could interact with one or more litters before weaning showed less aggression (Weary et al., 2002; Hessel et al., 2006; Kutzer et al., 2009) and skin lesions (Parratt et al., 2006) after weaning when kept with familiar piglets. This was likely because new hierarchies did not need to be established. Compared with piglets from single litter housing, piglets raised in MS systems also showed reduced aggression towards unfamiliar piglets in a social confrontation test (Hillmann et al., 2003) and towards unfamiliar piglets when mixed at weaning (Li and Wang, 2011; Bohnenkamp et al., 2013b). This reduced aggression may have several reasons. First, it has been suggested that pigs housed in large groups are forced to adopt a more tolerant strategy towards unfamiliar pigs (Samarakone and Gonyou, 2009). Second, the increased space and environmental complexity in MS systems may enhance piglet social development by better enabling expression of threatening and submissive behaviour (Lammers and Schouten, 1985) and by stimulating play behaviour (Bolhuis et al., 2005; Oostindjer et al., 2011). Play is thought to be important for the social development of piglets (Bekoff and Byers, 1981) and Arey and Sancha (1996) reported a ninefold increase in play behaviour in MS housing, compared with farrowing crates. Šilerová et al. (2010), however, found no difference in frequency of play between farms with straw-bedded pens for individually loose-housed sows and MS housing, possibly because of a lower contrast between housing types. In addition, increased social experience in itself also influences social development. For instance, social recognition was improved in piglets mingled twice pre-weaning, compared with unmingled piglets (Kanaan et al., 2012), and piglets mixed with another litter from 10 days until weaning formed a stable dominance hierarchy more rapidly when mixed with unfamiliar pigs at 7 weeks of age (D'Eath 2005), suggesting enduring effects of early social experiences on the development of social skills. Finally, MS housing may also positively affect adaptability in non-social challenging conditions. In a novel environment test, MS-reared piglets showed less activity, escape attempts, and vocalisations (Bünger et al., 2000; Hillmann et al., 2003), and more exploration (Hillmann et al., 2003) than piglets reared with individually housed sows.

In conclusion, piglet (social) development in MS housing is enhanced by early contact with non-littermates, and by increased space allowance and environmental complexity. This may facilitate adaptation to the social and physical environment after weaning. 


\subsubsection{Pre-weaning piglet feed intake, growth, and uniformity}

For piglets, adaptation to solid feed is important for a successful transition from lactation to the post-weaning period. Sufficient pre-weaning solid feed intake may improve early post-weaning feed intake and growth (e.g. Bruininx et al., 2002) and reduce the occurrence of diarrhoea after weaning (Yan et al., 2011). Creep feed intake during lactation, however, shows high individual variation in commercial practice (e.g. Bøe and Jensen, 1995). Group housing systems have the potential for increased piglet feed intake compared with single litter housing. First, motivation to ingest solid feed may be higher in systems with reduced contact between sows and piglets. Specifically, in GA systems, sows can evade nursing requests of piglets. Indeed, piglets from GA-housed sows consumed roughly twice as much feed as piglets in single litter housing pre-weaning (Rantzer et al., 1995; Weary et al., 2002). Second, feed intake is promoted if piglets can eat together with the sow (Oostindjer et al., 2010) and if piglets experienced with solid feed intake are present (Morgan et al., 2001). Therefore, MS-housed piglets may have more opportunities to learn from conspecifics, which resembles natural circumstances (Meynhardt, 1980). In the MS systems studied, however, piglets were mostly fed in a separate creep area (Table 2.1) with limited possibilities for vertical (sow-piglet) social learning. Rantzer et al. (1997) and Wattanakul et al. (1997) reported a lower preweaning feed intake in MS systems, compared with piglets from single litter housing $(0.4 \pm 0.1$ vs. $0.6 \pm 0.1 \mathrm{~kg} /$ piglet before weaning at 5 weeks p.p. and 18.4 vs. $24.6 \mathrm{~g} /$ day per piglet ( $\mathrm{SED}=3.4$ ) in the MS phase, respectively). Possibly, this may have resulted from the sudden introduction to the larger and more diverse MS area.

Even though pre-weaning experience with feed intake is important for adaptation to the post-weaning situation, piglet growth during lactation is mostly determined by milk intake. As previously discussed, compared with individual housing, nursing behaviour may be restricted or disrupted in group housing systems, which may be related to the relative transition in physical and social environment. Therefore, it might be expected that pre-weaning growth is lower and more variable in group housing systems with 'abrupt' transitions, compared with individual housing. As presented in Table 2.2, group housing during lactation either reduces (6 studies), does not affect (5 studies) or increases (2 studies) pre-weaning piglet growth compared with individual 
housing, and it seems that systems with more abrupt transitions from gestation to lactation and grouping have a relatively poor performance (Table 2.3). In line with this, the number of aggressive interactions between familiar sows after introduction to the MS area was found to be negatively correlated $(\mathrm{r}=-0.65)$ with piglets' daily gain during the MS phase (Dybkjær et al., 2003). Other system characteristics such as sow group size, the timing of grouping piglets, and the communal area available per litter do not seem to relate to preweaning piglet growth in the evaluated studies (Table 2.2). Uniformity in piglet growth until weaning in MS systems was either similar (Hultén et al., 1997; Wattanakul et al., 1997) or increased (Wattanakul et al., 1997; Bünger, 2002) compared with individual housing. Possibly, cross-suckling equalised the number of suckling piglets per sow and thereby piglet growth (Algers, 1991). Alternatively, light and weak piglets may have died earlier in MS housing (Hultén et al., 1997).

Pre-weaning feed intake in group housing systems could thus be improved in future systems by facilitation of social learning. Pre-weaning gain is likely affected by nursing behaviour, which may be influenced by the transition in social and physical environment.

\subsubsection{Post-weaning piglet feed intake, growth, and uniformity}

The post-weaning growth check often observed in piglets from conventional housing may be reduced or absent in piglets raised in group housing. Potential factors contributing to a better adaptation to the post-weaning situation are improved social skills, absence of mixing of piglets at weaning, increased experience with solid feed, or a higher weaning age (Berkeveld et al., 2009). For instance, piglets mingled with other litters during lactation gained 0.8 to $1.0 \mathrm{~kg}$ more in the 5 weeks post-weaning compared with housing without contact possibilities (Hessel et al., 2006; Kutzer et al., 2009).

Feed intake and growth of piglets reared in GA systems were improved the day after weaning (Weary et al., 2002) and 2 to 4 weeks after weaning (Rantzer et al., 1995) compared with piglets reared with individually housed sows. This likely relates to the observed higher pre-weaning feed intake. In both studies, all piglets remained in their system after weaning; however, but in the study by Rantzer et al. (1995) the piglets additionally gained access to the communal area and were thus mixed with unfamiliar litters. 


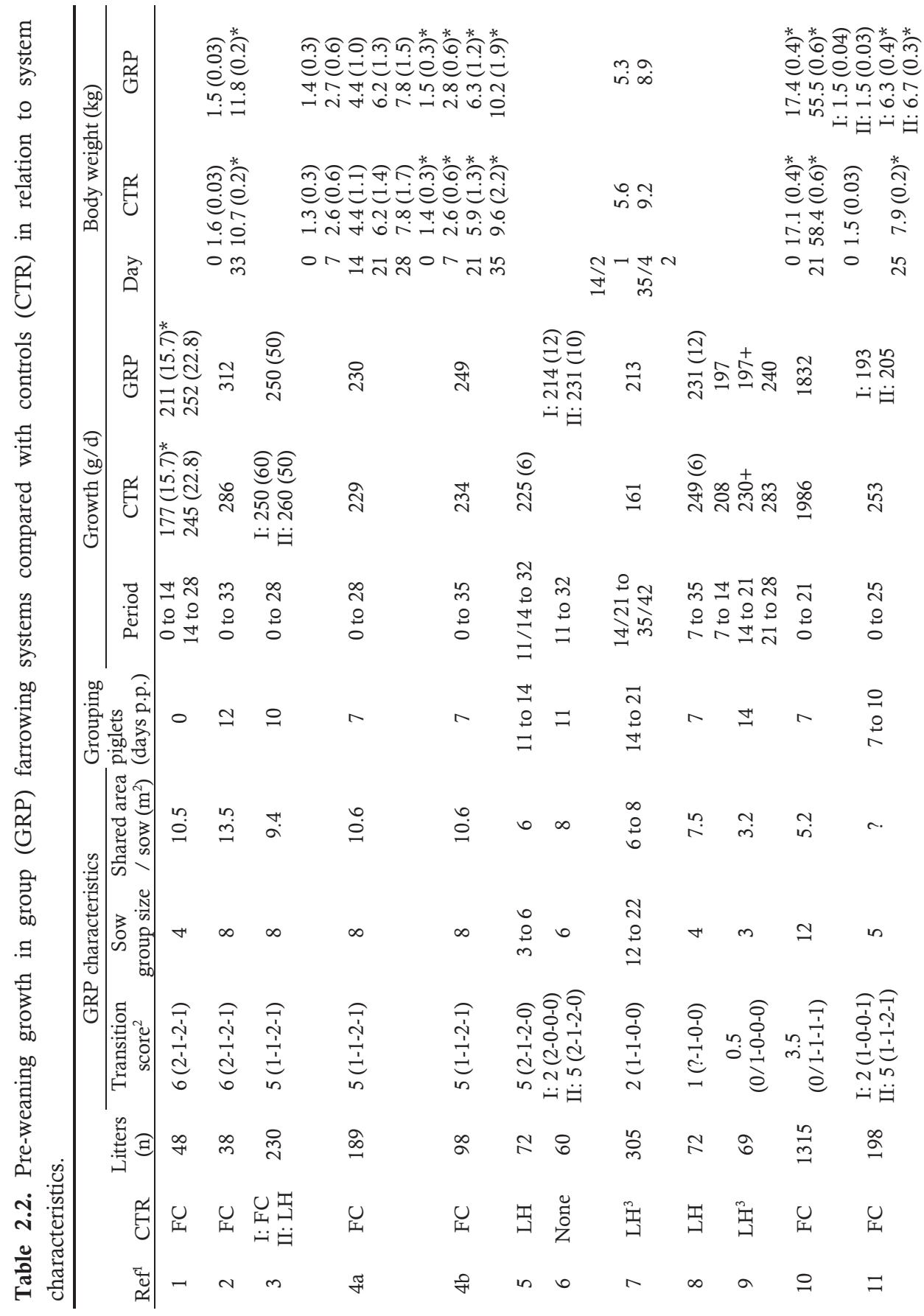




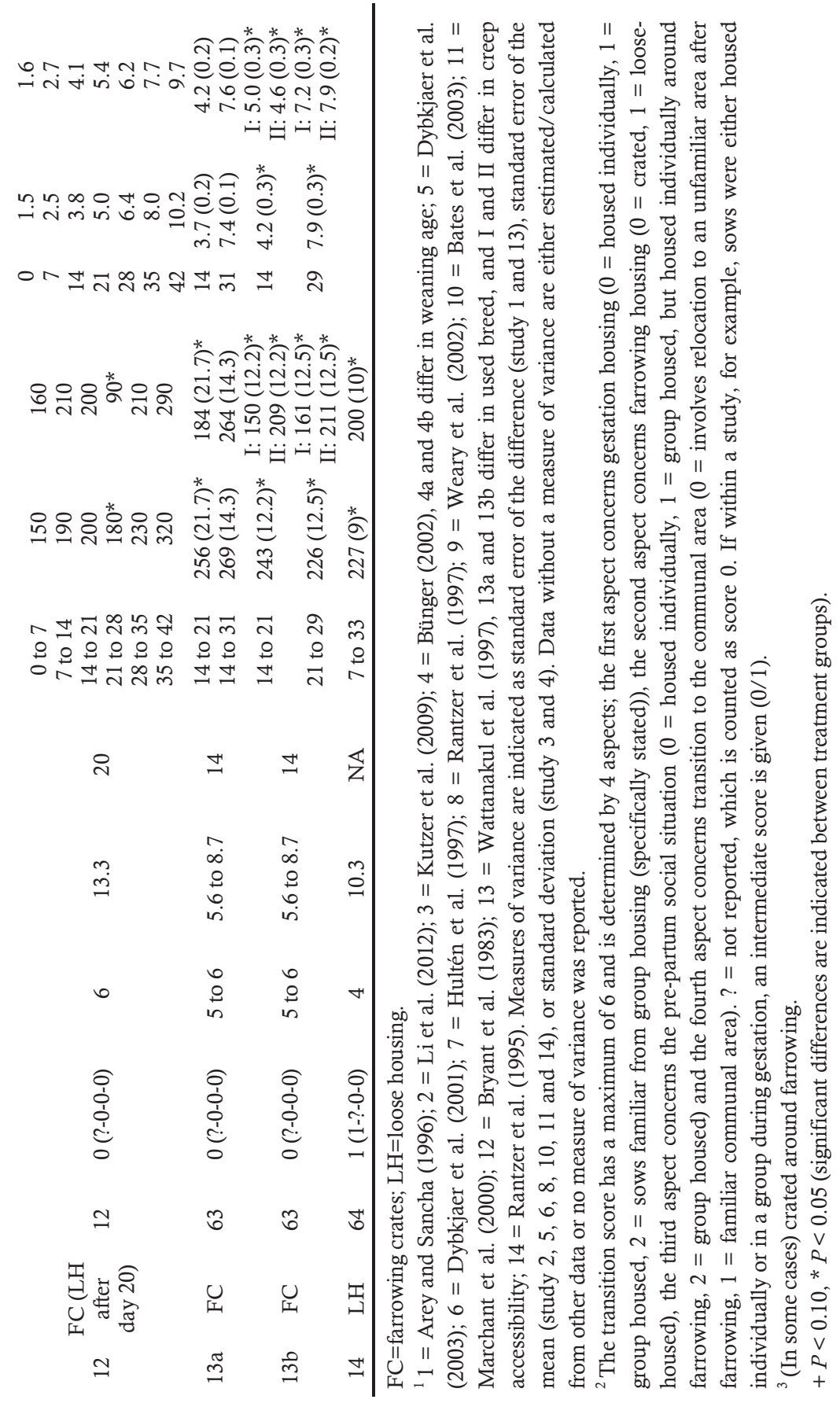


Table 2.3. Transition score per study investigating pre-weaning growth in group housing vs. individual housing for lactating sows ${ }^{1}$.

\begin{tabular}{llllllll}
\hline Transition score & $0-0.5$ & $1-1.5$ & $2-2.5$ & $3-3.5$ & $4-4.5$ & $5-5.5$ & 6 \\
\hline Study result & - & - & - & - & & - & + \\
& - & 0 & 0 & & & 0 & + \\
& - & & & & & 0 & \\
& & & & & & 0 & \\
\hline
\end{tabular}

${ }^{1}$ Each symbol represents 1 study, in which pre-weaning growth was higher $(+)$, similar $(0)$, or lower (-) in group housing than in individual housing. See Table 2.2 for explanation on transition scores. Reference $4 \mathrm{~b}$ is not included in the table, owing to a difference in birth weight between groups. Reference 11 is included twice because 2 different group housing systems were studied. Reference $13 \mathrm{a}$ and $13 \mathrm{~b}$ are combined.

MS studies greatly differ in the transition to the post-weaning environment, both regarding the MS-reared piglets and the piglets reared with individually housed sows, for instance, regarding available space and group size. This possibly partly explains the variable results in post-weaning feed intake and growth: in MS-reared pigs, feed intake was lower (in weeks 3 to 17 postweaning, Li et al., 2012), similar (about weeks 0 to 6 post-weaning, Korthals 2003), or higher (in the first week post-weaning, Wattanakul et al., 1997) compared with pigs reared with crated sows. MS-reared piglets showed a higher weight gain (first week, Wattanakul et al., 1997), similar weight gain (for at least the first 6 weeks post-weaning, Korthals, 2003; Li et al., 2012; Bohnenkamp et al., 2013b), or lower weight gain (the first 4 weeks, Rantzer et al., 1997) compared with piglets reared with individually housed sows. Only in the study of Hultén et al. (1997), all piglets were weaned by only removing the sows, and growth rate 2 weeks post-weaning was similar for MS-reared pigs and pigs reared with individually housed sows. This was possibly because weaning resulted in limited stress for both groups.

Concluding, post-weaning performance of piglets reared in MS housing benefits from limited changes in social and physical environment at weaning, as is the case for piglets reared with individually housed sows.

\subsubsection{Sow feeding behaviour and physical condition}

During lactation, energy requirements for milk are at a level that cannot be met by feed intake and, as a consequence, sows often lose substantial amounts of body weight during lactation, affecting subsequent reproductive performance. Sows in GA housing with 1 ad libitum feeder per sow consumed much feed 
during lactation, but less than sows in individual housing (11.3 vs. 12.9 $\mathrm{kg}$ /day), although no differences in body weight change were apparent. This is likely related to the observed lower nursing frequency (Weary et al., 2002). In other studies, feed intake and body condition are not comparable, as group housed sows were fed ad libitum during the MS phase, whereas individually housed sows were fed restrictedly.

When using 1 ad libitum feeder for 4 sows, the size of the MS area affected the daily pattern of feed intake but not the level of feed intake (Burke et al., 2000). The different eating strategies were possibly related to differences in possibilities to avoid more dominant sows. Aggression may increase around feeding (Goetz and Troxler, 1995) and Hultén et al. (1995b) observed that more primiparous sows, which often have a low social rank (Hoy et al., 2009), had skin wounds on the day of weaning on MS farms than on farms with individual housing. The percentage of multiparous sows with skin lesions was similar between housing systems. Therefore, space to avoid high ranking sows may be important to limit stress and ensure sufficient feed intake of primiparous sows in group housing with communal feeding.

In group housing systems, it is expected that sows are less affected by health problems related to lack of movement, such as leg problems and obstipation. In addition, it is expected that, owing to better regulation of nursing behaviour, sows in GA housing are less affected by udder and teat lesions than in individual housing. In MS housing, however, more competition at the udder occurs, which may result in higher levels of udder and teat damage. Sow health has not been investigated in the reviewed studies with GA systems; however, on MS farms, indeed fewer sows were culled because of locomotory problems (Hultén et al., 1998) and fewer sows suffered from scapular abscesses (3.0\% vs. $13.4 \%$ of sows), hoof abnormalities ( $4.6 \%$ vs. $9.1 \%$ of sows), and teat wounds and udder lesions than on farms with individually housed sows (Hultén et al., 1995b). This may, however, also be related to the higher amount of straw bedding that was available. The occurrence of mastitis did not differ between the 2 housing systems (Hultén et al., 1995b). Primiparous sows were, however, possibly more affected by piglet fights around suckling during the MS phase than multiparous sows, as indicated by the higher percentage of primiparous sows with teat wounds (Hultén et al., 1995b). In addition, Bohnenkamp et al. (2013a) reported that 9 of a total of 12 primiparous sows were removed from 
group housing because of injured teats, compared with 3 of 12 primiparous sows in farrowing crates.

In conclusion, MS housing may decrease the occurrence of locomotory problems, scapular abscesses, and teat and udder lesions. Primiparous sows may be at a disadvantage in MS systems, as indicated by a higher incidence of skin and teat damage compared with multiparous sows.

\subsubsection{Lactational ovulation and insemination}

Individually housed sows usually remain anoestrous until weaning (Quesnel and Prunier, 1995). Lactational ovulation can, however, be induced when suckling intensity is substantially reduced or by stress (reviewed by Langendijk et al., 2006). In MS housing, the occurrence of lactational ovulation ranged from $0 \%$ to $100 \%$ (Bryant et al., 1983; Hultén et al., 1995a; Wechsler, 1996; Wattanakul et al., 1997; Hultén et al., 2006; Kongsted and Hermansen, 2009), and was associated with a shorter nursing duration per nursing bout at 4 weeks p.p. and a lower number of piglets present at nursing between weeks 4 to 6 p.p. (Hultén et al., 2006), both of which indicate a reduced suckling intensity in these sows. Lactational ovulations were also associated with higher piglet weight gains between grouping and weaning at 7 weeks of age (Hultén et al., 2006), but this might also be a consequence of high piglet feed intake as piglets had access to sow feed from 2 weeks of age onwards. Sow weight loss, backfat thickness, and litter size did not differ between sows with and without lactational ovulation (Hultén et al., 1995a; Hultén et al., 2006). In addition, group size was unrelated to the occurrence of lactational ovulation (Hultén et al., 1995a). Weary et al. (2002) reported no lactational oestrus in a GA system.

In most systems, lactational ovulation is seen as a disadvantage, as it results in longer and more variable weaning-to-oestrus intervals if sows are not inseminated during lactation (Hultén et al., 1998; Hultén et al., 2006), which may be disadvantageous for managed batch farrowing systems. Furthermore, such variable intervals may result in less efficient oestrus detection and may explain why Hultén et al. (1998) found that MS-housed sows were relatively more often culled owing to repeat breeding or failure to farrow than individually housed sows ( $38.5 \%$ vs. $20.2 \%$ ). On the other hand, if sows are inseminated during lactational ovulation, farrowing rates and litter sizes are similar to those in sows inseminated after weaning (Soede et al., 2012). Thus, 
lactational inseminations might enhance sow performance in production systems with an extended lactation period (Kongsted and Hermansen, 2009), such as some MS and GA systems. If the timing of lactational ovulation is not predictable and synchronised, this will increase the age difference of the next batch of litters (Hultén et al., 1995a), which may increase the occurrence of cross-suckling (Wechsler, 1996; Maletínská and Špinka, 2001). Although lactational ovulations may be stimulated and more synchronised in case sows in oestrus are present (Pearce and Pearce, 1992), Hultén et al. (2006) found low synchrony in oestrus onset; $10 \%, 13 \%, 6 \%, 22 \%$, and $49 \%$ of ovulations occurred in the $3^{\text {rd }}, 4^{\text {th }}, 5^{\text {th }}, 6^{\text {th }}$, and last week of lactation, respectively. Kongsted and Hermansen (2009), however, placed sows in MS housing with boar exposure after 5 weeks of lactation in individual housing, which induced oestrus in $84 \%$ of sows within 1 week. Thus, possibilities exist to synchronise oestrus and ovulation in group housed sows, although little is known about the effects of oestrus behaviour of group housed lactating sows. Primiparous sows may, however, not show lactational ovulation (Hultén et al., 1995a) or at a low frequency (Hultén et al., 2006) and sows with a low social rank ovulated later during lactation after boar exposure (Kongsted and Hermansen 2009). Moreover, sows with high weight loss during lactation ovulated later during lactation (Hultén et al., 2006). On the other hand, timing of oestrus was unaffected by backfat thickness, litter size (Hultén et al., 1995a; Hultén et al., 2006; Kongsted and Hermansen, 2009), nursing duration at 4 weeks p.p., the number of piglets present at nursing between weeks 4 to 6 p.p., piglet weight gain between grouping and weaning (Hultén et al., 2006), and the number of weaned piglets (Kongsted and Hermansen 2009).

In conclusion, oestrus and ovulation may occur during lactation in sow group housing systems. Both the occurrence and synchrony of lactational ovulations can vary greatly between MS systems, and are influenced by sow parity, social rank, and boar presence. As lactational insemination can result in good reproductive performance, stimulation of synchronised ovulations may be a beneficial strategy for group housing systems.

\subsubsection{Sow suitability}

As group housed sows have more freedom to express behaviours than individually housed sows, the success of group housing systems may to a 
greater extent depend on sow behaviour. Specifically, the expression of maternal behaviour, for example attentiveness and lack of aggression towards piglets, is of great importance. In an on-farm study with 840 sows, $3.1 \%$ of culled MS-housed sows were removed because of inadequate maternal behaviour compared with $0.9 \%$ of culled individually housed sows (Hultén et al., 1998). Sows show much individual variation in maternal behaviour, and $\mathrm{Li}$ et al. (2010) found that individual sows showed consistency in piglet mortality over subsequent parities in MS housing. As maternal behaviour is partly genetically determined (Løvendahl et al., 2005), selecting sows with appropriate maternal behaviour may improve performance in group housing systems. Maternal behaviour can be affected by housing type (Baxter et al., 2011) and therefore selection of sows has to occur in a suitable environment, for example, not in farrowing crates. Moreover, the previous experiences of sows with housing systems influence their performance; sows that were raised in an MS system showed lower piglet mortality and tended to crush fewer piglets than sows with no previous experience in the system, resulting in 21.4 vs. 20.1 raised piglets per year (Wechsler, 1996). Finally, the physical condition of a sow also affects suitability for group housing. With more freedom of movement, a good leg condition is important. In addition, uncontrolled lying down behaviour is identified as a major risk for piglet crushing (Marchant et al., 2001). Thus, in order to be successful, group housing systems may require sows with good maternal behaviour, a good leg condition, and with previous experience with group housing. As not all sows are suitable for group housing during lactation, there may be a need for alternative housing for unfit sows.

\subsection{Concluding remarks}

The major strengths of group housing compared with individual housing during lactation include increased freedom of movement for sows and piglets with enhanced possibilities to express behaviours, for example related to maternal care, an improved social development of piglets, and a potential for increased pre-weaning piglet feed intake by enabling piglets to eat together with the sows, resulting in better adaptation of piglets to the post-weaning environment. These aspects also resemble the situation in a natural environment. Furthermore, insemination during lactation provides opportunities for an extended lactation period, which benefits piglets, while maintaining satisfactory sow performance. 
In sow group housing systems, most risk factors for poor sow and piglet performance seem to relate to altered nursing behaviour in relation to the level of change in social and physical environment during transitions around gestation, farrowing, grouping, and weaning. For good performance, it therefore seems important that these transitions are gradual or limited. In addition, it is beneficial if stockpersons are familiar with the system and it is recommended that sows with suitable physical and behavioural characteristics are used.

\subsection{Acknowledgements}

The authors gratefully acknowledge the Ministry of Economic Affairs for their financial support, Bas Kemp for his valuable comments on an earlier version of this paper and Bo Algers, Johannes Baumgartner, Emma Baxter, Anna-Lena Bohnenkamp, Anne-Marie Dalin, Sandra Edwards, Yuzhi Li, Rebecca Morrison, Lene Juul Pedersen, and Josef Troxler for kindly providing additional information on the systems and studies described. 


\subsection{References}

Algers B. 1991. Group housing of farrowing sows - health aspects on a new system. In: 7th International Congress on Animal Hygiene, 20-24 August 1991, Leipzig, Germany. p 851-857.

Arey D.S. and Sancha E.S. 1996. Behaviour and productivity of sows and piglets in a family system and in farrowing crates. Applied Animal Behaviour Science 50: 135-145.

Baxter E.M., Lawrence A.B., and Edwards S.A. 2011. Alternative farrowing systems: Design criteria for farrowing systems based on the biological needs of sows and piglets. Animal 5: 580-600.

Baxter E.M., Lawrence A.B., and Edwards S.A. 2012. Alternative farrowing accommodation: Welfare and economic aspects of existing farrowing and lactation systems for pigs. Animal 6: 96-117.

Bekoff M. and Byers J.A. 1981. A critical reanalysis of the ontogeny and phylogeny of mammalian social and locomotor play: an ethological hornet's nest. In: Immelman K., Barlow G.W., Petrinovic L., and Main M. (eds.) Behavioral Development. The Bielefeld Interdisciplinary Project. p 296-337. Cambridge University Press, Cambridge, England.

Bøe K. 1993. Maternal behaviour of lactating sows in a loosehousing system. Applied Animal Behaviour Science 35: 327-338.

Bøe K. and Jensen P. 1995. Individual differences in suckling and solid food intake by piglets. Applied Animal Behaviour Science 42: 183-192.

Bohnenkamp A.L., Meyer C., Müller K., and Krieter J. 2013a. Group housing with electronically controlled crates for lactating sows. Effect on farrowing, suckling and activity behavior of sows and piglets. Applied Animal Behaviour Science 145: 37-43.

Bohnenkamp A.L., Traulsen I., Meyer C., Müller K., and Krieter J. 2013 b. Comparison of growth performance and agonistic interaction in weaned piglets of different weight classes from farrowing systems with group or single housing. Animal 7: 309-315. 
Bolhuis J.E., Schouten W.G.P., Schrama J.W., and Wiegant V.M. 2005. Individual coping characteristics, aggressiveness and fighting strategies in pigs. Animal Behaviour. 2005; 69: 1085-1091.

Bruininx E.M.A.M., Binnendijk G.P., Van Der Peet-Schwering C.M.C., Schrama J.W., Den Hartog L.A., Everts H., and Beynen A.C. 2002. Effect of creep feed consumption on individual feed intake characteristics and performance of group-housed weanling pigs. Journal of Animal Science 80: 1413-1418.

Bryant M.J. and Rowlinson P. 1984. Nursing and suckling behaviour of sows and their litters before and after grouping in multi-accommodation pens. Animal Science 38: 277-282.

Bryant M.J., Rowlinson P., and Van der Steen H.A.M. 1983. A comparison of the nursing and suckling behaviour of group- and individually-housed sows and their litters. Animal Science 36: 445-451.

Bünger B. 2002. Effects of housing conditions of farrowing and nursing sows on development of piglets: Own studies and an evaluation of literature. Deutsche Tierarztliche Wochenschrift 109: 277-289.

Bünger B., Hillmann E., and Von Hollen F. 2000. Effects of housing conditions during farrowing and nursing of sows on growth and behaviour of piglets before and after weaning. Archiv fur Tierzucht 43: 196-202.

Burgwal-Konertz B. 1996. Das Saug- und Säugeverhalten bei der Gruppenhaltung abferkelnder und ferkelführender Sauen und ihren Würfen unter besonderer Berücksichtigung des Fremdsaugens. PhD, Thesis $\mathrm{PhD}$, University of Hohenheim, Stuttgart, Germany.

Burke J., Brooks P.H., Kirk J.A., and Eddison J.C. 2000. Daily food intakes and feeding strategies of sows given food ad libitum and allocated to two different space allowances in a communal farrowing system over parturition and during lactation. Animal Science 71: 547-559.

Dybkjær L., Olsen A.N.W., Møller F., Jensen K.H., and Giersing M. 2003. Effects of group size during pregnancy and introduction method on behaviour of relevance for piglet performance in multi-suckling pens. Acta Agriculturae Scandinavica - Section A: Animal Science 53: 83-91. 
Dybkjær L., Olsen A.N.W., Moøller F., and Jensen K.H. 2001. Effects of farrowing conditions on behaviour in multi-suckling pens for pigs. Acta Agriculturae Scandinavica - Section A: Animal Science 51: 134-141.

Goetz M. and Troxler J. 1995. Group housing of sows during farrowing and lactation. Transactions of the American Society of Agricultural Engineers 38: 14951500.

Hessel E.F., Reiners K., and Van Den Weghe H.F.A. 2006. Socializing piglets before weaning: Effects on behavior of lactating sows, pre- and postweaning behavior, and performance of piglets. Journal of Animal Science 84: 28472855.

Hillmann E., Von Hollen F., Bünger B., Todt D., and Schrader L. 2003. Farrowing conditions affect the reactions of piglets towards novel environment and social confrontation at weaning. Applied Animal Behaviour Science 81: 99-109.

Horrell I. 1997. The characterisation of suckling in wild boar. Applied Animal Behaviour Science 53: 271-277.

Horrell I. and Hodgson J. 1992. The bases of sow-piglet identification. 2. Cues used by piglets to identify their dam and home pen. Applied Animal Behaviour Science 33: 329-343.

Houwers H.W.J., Buré R.G., and Lokhorst C. 1996. Voermethode, bedrijfsvoering, stalinrichting en diergedrag bij geïntegreerde groepshuisvesting van zeugen, Wageningen: IMAG-DLO, 1996 (Rapport / Dienst Landbouwkundig Onderzoek, Instituut voor Milieu- en Agritechniek 96-04).

Hoy S., Bauer J., Borberg C., Chonsch L., and Weirich C. 2009. Investigations on dynamics of social rank of sows during several parities. Applied Animal Behaviour Science 121: 103-107.

Hultén F., Dalin A.M., Lundeheim N., and Einarsson S. 1995a. Ovulation frequency among sows group-housed during late lactation. Animal Reproduction Science 39: 223-233.

Hultén F., Lundeheim N., Dalin A.M., and Einarsson S. 1995b. A field study on group housing of lactating sows with special reference to sow health at weaning. Acta Veterinaria Scandinavica 36: 201-212. 
Hultén F., Lundeheim N., Dalin A.M., and Einarsson S. 1997. Pre- and Post-Weaning Piglet Performance, Sow Food Intake and Change in Backfat Thickness in a Group-Housing System for Lactating Sows. Acta Veterinaria Scandinavica 38: 119-133.

Hultén F., Lundeheim N., Dalin A.M., and Einarsson S. 1998. Reproductive Performance among Sows Group-Housed during Late Lactation. Acta Veterinaria Scandinavica 39: 237-250.

Hultén F., Wallenbeck A., and Rydhmer L. 2006. Ovarian activity and oestrous signs among group-housed, lactating sows: Influence of behaviour, environment and production. Reproduction in Domestic Animals 41: 448-454.

Illmann G., Pokorná Z., and Špinka M. 2007. Allosuckling in domestic pigs: Teat acquisition strategy and consequences. Applied Animal Behaviour Science 106: $26-38$.

Jensen P. 1986. Observations on the maternal behaviour of free-ranging domestic pigs. Applied Animal Behaviour Science 16: 131-142.

Jensen P. 1988. Maternal behaviour and mother-young interactions during lactation in free-ranging domestic pigs. Applied Animal Behaviour Science 20: 297-308.

Jensen P. and Recén B. 1989. When to wean - Observations from free-ranging domestic pigs. Applied Animal Behaviour Science 23: 49-60.

Jensen P. and Redbo I. 1987. Behaviour during nest leaving in free-ranging domestic pigs. Applied Animal Behaviour Science 18: 355-362.

Johnson A.K. and Marchant-Forde J.N. 2009. Welfare of Pigs in the Farrowing Environment. In: Marchant-Forde J.N. (ed.) The welfare of pigs. Animal welfare No. 7. p 141-188. Springer, Dordrecht, Netherlands.

Kanaan V.T., Lay Jr D.C., Richert B.T., and Pajor E.A. 2012. Increasing the Frequency of Co-Mingling Piglets During the Lactation Period Alters the Development of Social Behavior Before and After Weaning. Journal of Applied Animal Welfare Science 15: 163-180.

Kongsted A.G. and Hermansen J.E. 2009. Induction of lactational estrus in organic piglet production. Theriogenology 72: 1188-1194.

Korthals R. 2003. Pig performance comparing a production system using large static groups formed during lactation to a production system using sized and 
resorted groups in nursery and finisher. Journal of Swine Health and Production 11: 19-24.

Kutzer T., Bünger B., Kjaer J.B., and Schrader L. 2009. Effects of early contact between non-littermate piglets and of the complexity of farrowing conditions on social behaviour and weight gain. Applied Animal Behaviour Science 121: $16-24$.

Lammers G.J. and Schouten W.G.P. 1985. Effects of pen size during rearing on later agonistic behaviour in piglets. Netherlands Journal of Agricultural Science 33: 307-309.

Langendijk P., Soede N.M., and Kemp B. 2006. Effects of boar stimuli on the follicular phase and on oestrous behaviour in sows. Society of Reproduction and Fertility supplement 62: 219-230.

Li Y., Johnston L., and Hilbrands A. 2010. Pre-weaning mortality of piglets in a bedded group-farrowing system. Journal of Swine Health and Production 18: 75-80.

Li Y. and Wang L. 2011. Effects of previous housing system on agonistic behaviors of growing pigs at mixing. Applied Animal Behaviour Science 132: 20-26.

Li Y.Z., Wang L.H., and Johnston L.J. 2012. Effects of farrowing system on behavior and growth performance of growing-finishing pigs. Journal of Animal Science 90: 1008-1014.

Løvendahl P., Damgaard L.H., Nielsen B.L., Thodberg K., Su G., and Rydhmer L. 2005. Aggressive behaviour of sows at mixing and maternal behaviour are heritable and genetically correlated traits. Livestock Production Science 93: 73-85.

Maletínská J. and Špinka M. 2001. Cross-suckling and nursing synchronisation in group housed lactating sows. Applied Animal Behaviour Science 75: 17-32.

Marchant J.N., Broom D.M., and Corning S. 2001. The influence of sow behaviour on piglet mortality due to crushing in an open farrowing system. Animal Science 72: 19-28.

Marchant J.N., Rudd A.R., Mendl M.T., Broom D.M., Meredith M.J., Corning S., and Simmins P.H. 2000. Timing and causes of piglet mortality in alternative and conventional farrowing systems. Veterinary Record 147: 209-214. 
Melotti L., Oostindjer M., Bolhuis J.E., Held S., and Mendl M. 2011. Coping personality type and environmental enrichment affect aggression at weaning in pigs. Applied Animal Behaviour Science 133: 144-153.

Meynhardt H. 1980. Schwarzwild-Report: Vier Jahre unter Wildschweinen. Verlag J. Neumann-Neudamm, Berlin, Germany.

Morgan C.A., Lawrence A.B., Chirnside J., and Deans L.A. 2001. Can information about solid food be transmitted from one piglet to another? Animal Science 73: 471-478.

Olsen A.N.W., Dybkjær L., and Vestergaard K.S. 1998. Cross-suckling and associated behaviour in piglets and sows. Applied Animal Behaviour Science 61: 13-24.

Oostindjer M., Bolhuis J.E., Mendl M., Held S., Gerrits W., Van den Brand H., and Kemp B. 2010. Effects of environmental enrichment and loose housing of lactating sows on piglet performance before and after weaning. Journal of Animal Science 88: 3554-3562.

Oostindjer M., van den Brand H., Kemp B., and Bolhuis J.E. 2011. Effects of environmental enrichment and loose housing of lactating sows on piglet behaviour before and after weaning. Applied Animal Behaviour Science 134: $31-41$.

Parratt C.A., Chapman K.J., Turner C., Jones P.H., Mendl M.T., and Miller B.G. 2006. The fighting behaviour of piglets mixed before and after weaning in the presence or absence of a sow. Applied Animal Behaviour Science 101: 54-67.

Pearce G.P. and Pearce A.N. 1992. Contact with a sow in oestrus or a mature boar stimulates the onset of oestrus in weaned sows. Veterinary Record 130: 5-9.

Pedersen L.J., Studnitz M., Jensen K.H., and Giersing A.M. 1998. Suckling behaviour of piglets in relation to accessibility to the sow and the presence of foreign litters. Applied Animal Behaviour Science 58: 267-279.

Quesnel H. and Prunier A. 1995. Endocrine bases of lactational anoestrus in the sow. Reproduction Nutrition Development 35: 395-414.

Rantzer D., Svendsen J., and Weström B. 1995. Weaning of pigs raised in sowcontrolled and in conventional housing systems, 1: Description of systems, production and bacteriology. Swedish Journal of Agricultural Research 25. 
Rantzer D., Svendsen J., and Weström B. 1997. Weaning of pigs in group housing and in conventional housing systems for lactating sows. Swedish Journal of Agricultural Research 27: 23-31.

Roulin A. 2002. Why do lactating females nurse alien offspring? A review of hypotheses and empirical evidence. Animal Behaviour 63: 201-208.

Rushen J., Ladewig J., and de Passillé A.M.B. 1995. A novel environment inhibits milk ejection in the pig but not through HPA activity. Applied Animal Behaviour Science 45: 53-61.

Samarakone T.S. and Gonyou H.W. 2009. Domestic pigs alter their social strategy in response to social group size. Applied Animal Behaviour Science 121: 8-15.

Šilerová J., Špinka M., Šárová R., and Algers B. 2010. Playing and fighting by piglets around weaning on farms, employing individual or group housing of lactating sows. Applied Animal Behaviour Science 124: 83-89.

Šilerová J., Špinka M., Šárová R., Slámová K., and Algers B. 2006. A note on differences in nursing behaviour on pig farms employing individual and group housing of lactating sows. Applied Animal Behaviour Science 101: 167-176.

Soede N.M., Laurenssen B., Abrahamse-Berkeveld M., Gerritsen R., Dirx-Kuijken N., Langendijk P., and Kemp B. 2012. Timing of lactational oestrus in intermittent suckling regimes: Consequences for sow fertility. Animal Reproduction Science 130: 74-81.

Valros A.E., Rundgren M., Špinka M., Saloniemi H., Rydhmer L., and Algers B. 2002. Nursing behaviour of sows during 5 weeks lactation and effects on piglet growth. Applied Animal Behaviour Science 76: 93-104.

Wattanakul W., Edwards S.A., Stewart A.H., and English P.R. 1998. Effect of familiarity with the environment on the behaviour and performance response of sows and piglets to grouping during lactation. Applied Animal Behaviour Science 61: 25-39.

Wattanakul W., Sinclair A.G., Stewart A.H., Edwards S.A., and English P.R. 1997. Performance and behaviour of lactating sows and piglets in crate and multisuckling systems: A study involving European White and Manor Meishan genotypes. Animal Science 64: 339-349. 
Weary D.M., Pajor E.A., Bonenfant M., Fraser D., and Kramer D.L. 2002. Alternative housing for sows and litters Part 4. Effects of sow-controlled housing combined with a communal piglet area on pre- and post-weaning behaviour and performance. Applied Animal Behaviour Science 76: 279-290.

Wechsler B. 1996. Rearing pigs in species-specific family groups. Animal Welfare 5: 25-35.

Worobec E.K., Duncan I.J.H., and Widowski T.M. 1999. The effects of weaning at 7 , 14 and 28 days on piglet behaviour. Applied Animal Behaviour Science 62: 173-182.

Wülbers-Mindermann M. 1992. Characteristics of cross-suckling piglets reared in a group housing system. Sveriges Lantbruksuniversitet, Institutionen för husdjurshygien, Skara, Sweden.

Yan L., Jang H.D., and Kim I.H. 2011. Effects of varying creep feed duration on preweaning and post-weaning performance and behavior of piglet and sow. Asian-Australasian Journal of Animal Sciences 24: 1601-1606. 



\section{CHAPTER 3}

Development of piglets raised in a new multi-litter housing system vs. conventional single-litter housing until 9 weeks of age

S.E. van Nieuwamerongen ${ }^{1^{*}}$, N.M. Soede ${ }^{1}$, C.M.C. van der PeetSchwering ${ }^{2}$, B. Kemp ${ }^{1}$, J.E. Bolhuis ${ }^{1}$

${ }^{1}$ Adaptation Physiology Group, Department of Animal Sciences, Wageningen University, PO Box 338, 6700 AH Wageningen, The Netherlands;

${ }^{2}$ Wageningen UR Livestock Research, PO Box 338, 6700 AH Wageningen, The Netherlands.

*Corresponding author: S.E. van Nieuwamerongen at Sofie.vannieuwamerongen@wur.nl.

Journal of Animal Science (2015) 93: 5442 - 5454 


\subsection{Abstract}

This study compared the development until 9 weeks of age of piglets raised in either a multi-litter (ML) system or a conventional single-litter (SL) system. The ML system consisted of a multi-suckling system with 5 sows and their litters before weaning, followed by housing in a pen with enrichment in a group of 40 piglets after weaning. In the SL system, piglets were housed with a crated sow before weaning, followed by post-weaning housing in groups of 10 litter-mates in a standard pen. Fifty litters were used in 5 batches and piglets were weaned at 4 weeks of age. Pre-weaning mortality was higher in the ML system than in the SL system $(3.22 \pm 0.42$ vs. $1.52 \pm 0.25$ piglets per litter, $P<$ 0.01 ), mainly due to crushing before grouping of litters. Litter size at grouping did not differ between systems. ML piglets showed more feed-directed behaviour at 2 weeks of age ( $6.80 \pm 0.96$ vs. $2.35 \pm 0.59, P<0.01)$, suggesting an earlier start of feed exploration, possibly due to social learning from the floor-fed sows and other piglets. Moreover, before weaning, ML piglets showed less damaging oral manipulation (e.g. tail biting) than SL piglets (1.4 \pm 0.2 vs. $3.6 \pm 0.3$ freq $/ \mathrm{h}, P<0.001$ ), which was likely related to the more enriched environment in the ML system. After weaning, ML piglets ate $81 \%$ more feed between days 1 and $2(0.29 \pm 0.02$ vs. $0.16 \pm 0.03 \mathrm{~kg} /$ piglet, $P<$ $0.01)$ and had an $82 \%$ higher weight gain until day 5 than SL piglets $(1.35 \pm$ 0.21 vs. $0.75 \pm 0.17 \mathrm{~kg}, P<0.05)$ despite a similar weaning weight (ML: $8.4 \pm$ $0.2 \mathrm{~kg}, \mathrm{SL}: 8.3 \pm 0.2 \mathrm{~kg}$ ). Within the first 2 weeks after weaning, ML piglets had a lower faecal consistency score $(0.27 \pm 0.03$ vs. $0.39 \pm 0.03, P<0.05)$, indicating a lower occurrence of diarrhoea compared with SL piglets. Over the entire 5 -week post-weaning phase, ML piglets had a $24 \%$ higher weight gain $(P$ $<0.05)$, showed more play behaviour $(4.0 \pm 0.3$ vs. $2.8 \pm 0.3 \mathrm{freq} / \mathrm{h}, P<0.05)$, and less damaging oral manipulation $(1.8 \pm 0.3$ vs. $3.5 \pm 0.4$ freq $/ \mathrm{h}, P<0.01)$ than SL piglets. These results are probably explained by a combination of the differences in pre-weaning development, early post-weaning performance, and post-weaning environment, with a larger and more diverse social group and more physical enrichment in the ML pen. To summarise, provided that preweaning mortality can be reduced, the ML system seems promising for raising robust piglets with better welfare, indicated by a better pre-weaning behavioural development, improved transition to the post-weaning phase, and better post-weaning performance. 
Key words: behaviour, development, group housing, multi-suckling system, pigs, weaning

\subsection{Introduction}

Recently, animal welfare concerns have led to a ban of individual confinement of sows during the major part of gestation in the EU (Council Directive $2001 / 88 / \mathrm{EC}$ ), and also in other countries, including the United States, there is a move toward group housing of gestating sows (Schau et al., 2013). During farrowing and lactation, however, most sows are individually housed in crates, and the transfer from group housing to confinement may impair welfare (Weng et al., 2009). Alternative farrowing systems that better meet behavioural needs of sows and that provide a better transition from group housing during gestation, such as multi-suckling (MS) systems, have received increasing attention (e.g. Bohnenkamp et al., 2013; Phillips et al., 2014). MS systems may not only affect sow welfare, but can also influence piglet welfare and development. Besides risk factors, such as restlessness around nursing and increased piglet mortality after grouping of litters, MS systems have several potential benefits (reviewed by Van Nieuwamerongen et al., 2014). For instance, MS housing may promote social development by providing contact between multiple sows and litters, similar to the social structure seen in feral pigs and wild boar. Moreover, an opportunity of MS housing that has received little attention so far is the stimulation of pre-weaning ingestive behaviour through learning from other piglets and sows, which may positively affect postweaning performance (Oostindjer et al., 2011a). Another advantage of MS systems is the possibility of keeping piglets in larger groups, without a need for the post-weaning mixing of unfamiliar piglets, which is one of the simultaneous stressors weanling piglets often have to cope with (Weary et al., 2008). Given these potential benefits of MS systems for piglet performance compared with conventional housing, we developed a multi-litter system for piglets between 0 and 9 weeks of age. The aim of this study was to compare the pre- and post-weaning development of piglets raised in the multi-litter system with the development of piglets raised in a conventional single-litter system. 


\subsection{Materials and methods}

\subsubsection{Animals and housing}

The experiment was approved by the Animal Care and Use Committee of Wageningen University. Five consecutive batches of 10 multiparous Topigs 20 sows and their litters (Tempo x Topigs 20) were studied at the animal facilities of Swine Innovation Centre Sterksel, the Netherlands. Per batch, 5 sows were allocated to multi-litter (ML) housing and 5 sows were allocated to single-litter (SL) housing. Multi-litter housing consisted of a multi-suckling system with 5 sows and their litters before weaning, followed by housing in a pen with enrichment in a group of 40 piglets after weaning. In SL housing, piglets were housed with a crated sow before weaning, followed by post-weaning housing in groups of 10 litter-mates in a standard pen.

\subsubsection{General management}

\section{Pre-weaning}

One week before expected farrowing, sows were transferred to either ML or SL housing, balanced for parity $(3.1 \pm 0.1)$ and expected farrowing date. Per batch, sows allocated to ML housing originated from the same stable group during gestation. In both treatments, piglets were ear tagged within 24 hours post-partum (p.p.) and, if needed, cross-fostered to and from sows outside the experiment. Litter sizes were equalised according to the number of functional teats available per sow, with a maximum of 14 piglets per litter. Per litter there were $14.87 \pm 0.46$ live born piglets and $0.65 \pm 0.12$ stillborn piglets and $0.52 \pm$ 0.45 piglets were cross-fostered to sows outside the experiment. Piglets were tail docked and received an iron injection within 4 days after birth. Male piglets were not castrated. Sows received a commercial diet containing 9.5 MJ of NE, $14.9 \%$ crude protein, and $0.76 \%$ ileal digestible lysine. Pre-starter creep feed (11.6 MJ of NE, $17.5 \%$ crude protein, and $1.17 \%$ ileal digestible lysine) was provided to the piglets in round feeders (diameter $28 \mathrm{~cm}$ ) twice daily from 12 days after the expected farrowing date. On days 21 and 22 pre-starter was gradually mixed with weaner feed (9.9 MJ of NE, 16.0\% crude protein, and $0.99 \%$ ileal digestible lysine), after which only weaner feed was provided. Water was available ad libitum. A circovirus-mycoplasma vaccine (Boehringer Ingelheim) was administered to the piglets in the third week of life. Animal 
health was checked twice daily and animals were treated with antibiotics or analgesics if necessary. Litters were weaned at $27.1 \pm 0.3$ days of age.

\section{Post-weaning}

Per batch and per system, 40 healthy piglets were selected for further study (10 piglets from 4 litters each) with a 1:1 male:female ratio. The median weights of the selected piglets were representative of the median weights of their own litter and of all 5 experimental litters per batch. ML and SL piglets were placed in 2 separate but adjacent rooms. Use of these rooms alternated between batches. Flooring consisted of $40 \%$ heated solid concrete and $60 \%$ metal slats. Space allowance was equal in both systems $\left(0.43 \mathrm{~m}^{2}\right.$ per piglet). Water was continuously available and commercial feed was provided ad libitum in feeders with 1 feeding place per 5 piglets. Creep feeders that were familiar from the pre-weaning phase were provided during days 0 to 5 post-weaning ( 1 per 10 piglets). The weaner feed was provided until day 9 post-weaning. On days 10 and 12 post-weaning, weaner feed was mixed with a starter diet $(9.7 \mathrm{MJ}$ of $\mathrm{NE}$, $15.6 \%$ crude protein, and $0.94 \%$ ileal digestible lysine), after which only the starter diet was provided. Animal health was checked twice daily and piglets were treated with antibiotics or analgesics if necessary.

\subsubsection{Multi-litter housing}

\section{Pre-weaning}

ML housing consisted of 5 farrowing pens connected to a communal area (Figure 3.1). Sows could access all areas from the moment of entry in the system. The farrowing pens were $2.4 \times 3.0 \mathrm{~m}$ each and contained a feed trough for the sow, a water nipple for the sow and piglets, and a piglet nest of $0.6 \times 1.3$ $\mathrm{m}$ with heated solid flooring. The sows could move around freely in the farrowing pens and were not confined in a crate. Each pen had 3 sloped walls to prevent piglet crushing. Each pen contained a mat of $1.1 \times 2.5 \mathrm{~m}$. The remaining floor consisted of concrete with $5 \%$ perforations. Before entry in the system, 5 hessian sacks were provided per pen as nesting material. From day 2 p.p., 2 handfuls of long-stemmed straw were provided daily per pen. The adjacent communal area was divided in an area for feeding $\left(7 \mathrm{~m}^{2}\right.$ solid concrete), defecating/urinating (12.5 $\mathrm{m}^{2}$ metal slats with 2 water nipples), and lying $\left(31.5 \mathrm{~m}^{2} 5 \%\right.$ perforated concrete with a $2.0 \times 3.2 \mathrm{~m}$ mat). Five hessian 


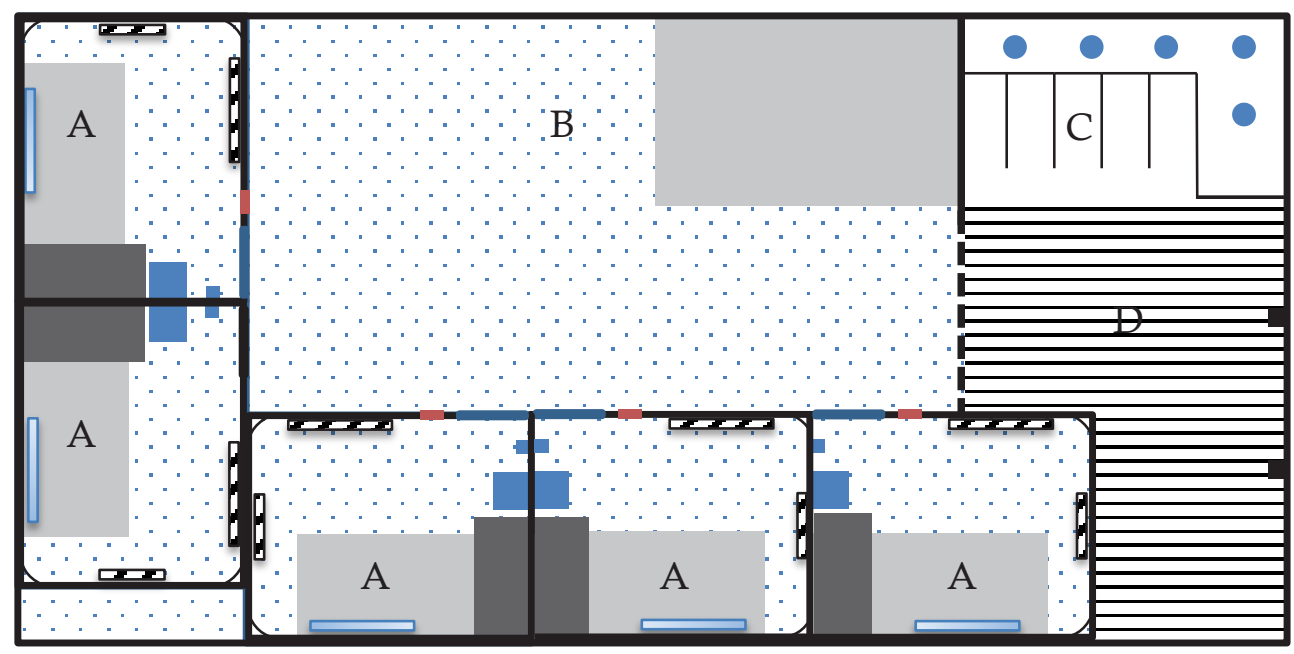

Figure 3.1. Layout of the multi-suckling system $(13.4 \times 6.6 \mathrm{~m})$. The system has 5 farrowing pens (A - with piglet nest, sow feeder, water nipple, and sloped walls) connected to a communal area with a lying area (B), feeding area (C - with 5 sow feeding places surrounded by a piglet area with 5 piglet feeders), and a dunging area (D - with 2 water nipples).

sacks and 5 ropes were attached to the partitions surrounding the resting area. Hessians sacks and ropes were replaced if worn and at least weekly. The communal feeding area contained 5 feeding places for the sows (separated by 1 $\mathrm{m}$ long horizontal metal bars) surrounded by an area with 5 round piglet feeders only accessible to the piglets. Sows were prevented from entering the piglet area by horizontal metal bars. Sows were locked in their assigned farrowing pen daily between 16:30 and 07:30 h from entry in the system until farrowing had ended. From that moment onwards, sows could freely access all farrowing pens and communal areas again, 24 hours per day. Piglets were kept inside their farrowing pen using a $31 \mathrm{~cm}$ high piglet barrier that sows could step over at the entrance. Piglets were given access to the whole system when the youngest litter was 6 days of age (average grouping age was $6.8 \pm 0.1$ days). This was done by removing the barrier and a piglet hatch $(0.4 \times 0.3 \mathrm{~m})$ from the farrowing pen. The sows were manually floor fed twice daily in the communal area, and piglets could eat from the sow feed and from creep feed provided in the piglet feeders. Sows were fed in their own farrowing pen only in the evenings before farrowing and if they would not leave their pen to eat in the communal area in the first days after farrowing. Until farrowing, sows received $2.8 \mathrm{~kg}$ of feed daily. Thereafter, daily feed provisioning was $2.5 \mathrm{~kg}$ of feed, which gradually increased to a maximum around $7.5 \mathrm{~kg}$ from day 10 of 
lactation onward. Ambient temperature $\left( \pm \mathrm{SD}\right.$ ) was $20.8 \pm 1.8^{\circ} \mathrm{C}$ in the communal area, $21.6 \pm 1.8^{\circ} \mathrm{C}$ in the farrowing pens, and $24.9 \pm 1.0^{\circ} \mathrm{C}$ in the piglet nests.

\section{Post-weaning}

Per batch, 40 piglets were housed in 1 pen of $6.7 \times 2.6 \mathrm{~m}$. The pen contained 4 feeders with 8 feeding places, 4 water nipples, 4 chains hanging from the ceiling with either a chew toy or plastic rolls attached, and 4 hessian sacks and 8 ropes attached to the front pen wall. Additionally, piglets received twice daily 4 to 6 handfuls of long-stemmed straw (increasing with age). Ambient temperature $( \pm \mathrm{SD})$ was $25.2 \pm 1.4^{\circ} \mathrm{C}$. Lights were on from 07:00 to $18: 00 \mathrm{~h}$ (with the exception of batch 1 , in which lights were on continuously due to a technical error).

\subsubsection{Single-litter housing}

\section{Pre-weaning}

The farrowing crates measured $1.9 \times 0.6 \mathrm{~m}$ and were contained in a pen of 1.7 $\mathrm{x} 2.5 \mathrm{~m}$. The floor consisted of metal slats under the crate, a solid floor of $1.5 \mathrm{x}$ $0.3 \mathrm{~m}$ with a heat lamp for the piglets, and plastic slats in the remaining area. The sow had a feed trough and drinking nipple available. Until farrowing, sows received $2.8 \mathrm{~kg}$ of feed daily. Thereafter, daily feed provisioning was 2.5 $\mathrm{kg}$ of feed, which gradually increased to a maximum around $7.5 \mathrm{~kg}$ given from day 15 of lactation onward. The piglets had access to a separate drinking nipple and creep feed was provided in a feeder located at the posterior side of the sow. One day before expected farrowing, sows received a hessian sack as nesting material. A rope and a plastic roll were available during the whole pre-weaning period as enrichment for sow and piglets. Ropes were replaced if worn and at least weekly. Average ambient temperature during the whole farrowing phase was $24.3 \pm 1.5^{\circ} \mathrm{C}$.

\section{Post-weaning}

Per batch, 40 piglets were housed in 4 pens of $1.7 \times 2.6 \mathrm{~m}$ in groups of 10 littermates; no mixing with unfamiliar piglets occurred at weaning. Pen partitions consisted of $60 \%$ bar fencing and $40 \%$ solid wall. Each pen contained 1 chew toy hanging from the ceiling, 1 water nipple, and 1 feeder with 2 feeding 
places. The 4 pens were located in 1 room. Ambient temperature was $26.7 \pm$ $1.0^{\circ} \mathrm{C}$. Lights were on from $07: 00$ to $18: 00 \mathrm{~h}$ (with the exception of batch 2 , in which lights were on continuously due to a technical error).

\subsubsection{Measurements}

\section{Behavioural observations}

Piglets were marked individually on the day before observations using hair dye (pre-weaning) or stock marker spray (post-weaning) to allow individual identification. Pre-weaning, 2 males and 2 females were observed per litter. The focal piglets had birth weights closest to the median birth weight of the litter present on day 5 after expected farrowing. Frequency of play, manipulative, aggressive, and feed-directed behaviour (Appendix 1) was recorded by 2 observers on days 15 and 26 p.p. using continuous behaviour sampling. Observations were performed between 08:00 and 16:00 h during 5 time blocks. Within each block, the 4 focal piglets of 1 litter were observed for 10 minutes, i.e. 50 minutes of observations per piglet per day. Post-weaning, all 80 piglets per batch were observed. Frequency of play, manipulative, and aggressive behaviour (Appendix 1) was recorded on days 1, 6, 14, and 34 postweaning using continuous behaviour sampling. Observations were carried out between 08:00 and 17:00 $\mathrm{h}$ during 4 time blocks. Within each block, the piglets of 1 litter were observed for 10 minutes, i.e. 40 minutes of observations per piglet per day. Per 10 minute observation, either $5 \mathrm{ML}$ piglets or $10 \mathrm{SL}$ piglets were observed simultaneously per observer because identification of individual piglets was more difficult in the larger ML group. All observations within and between time blocks were balanced for housing system and observer.

Body weight, feed intake, eater classification, damage scores, and faecal consistency scores

Piglets were weighed within 24 hours after birth, the day before weaning, and days $2,5,13$, and 35 post-weaning. Post-weaning feed intake at pen level was determined by registering the amount of feed provided and weighing the leftover feed between days 0 and 1, 1 and 2, 2 and 5, 5 and 13, and 13 and 35 . On the day before weaning, each piglet was classified as 'eater' or 'non-eater' based on a faecal sample taken from the rectum with a cotton swab (eater = brown faeces with coarse structure; non-eater $=$ yellow faeces with smooth 
structure). Pilot data using 52 piglets from 6 litters have shown that the sensitivity of this method is $96.2 \%$ and the specificity is $73.5 \%$. Damage on snout (pre-weaning only), carpal joints, ears, tail, and skin was scored individually using the protocols in (Appendix 2) on the day before weaning and day 33 after weaning. The number of skin lesions was counted on days 2 and 5 post-weaning, as a proxy measure of aggressive acts given and received (Turner et al., 2006). A faecal consistency score was given to all piglets during days 0 to 14 post-weaning, where the anal area of each piglet was visually inspected and scored as containing either no faeces (considered as normal faecal consistency), pasty faeces, or watery faeces.

\section{Blood sampling}

On days 0 (after sow removal and before relocation), 13, and 35 post-weaning, blood samples were taken using venepuncture of the jugular vein while piglets were restrained in supine position by 2 people. Samples were taken from selected piglets of which pre-weaning behaviour was observed $(N=32$ per batch). Blood was collected in 1 EDTA tube and 1 heparin tube per piglet. EDTA tubes were stored at room temperature and blood was used to determine leukocyte concentration as previously described by Reimert et al. (2014). Heparin tubes were stored on ice until centrifuging at $1,300 \mathrm{~g}$ at $4^{\circ} \mathrm{C}$. Plasma was stored at $-20^{\circ} \mathrm{C}$ (for antibody titers) or $-80{ }^{\circ} \mathrm{C}$ (haptoglobin concentration) until analysis. IgG and IgM antibody titers binding Keyhole Limpet Hemocyanin (KLH) were determined using an ELISA. Haptoglobin concentration was determined using a commercial second-generation haptoglobin kit with a colorimetric assay as described by Reimert et al. (2014).

\subsubsection{Statistical analysis}

Data were analysed with SAS 9.2 (SAS Inst. Inc., Cary, NC). Variables were averaged per housing system and batch before analysis, using 5 litters (before weaning) or 40 piglets (after weaning) as 1 experimental unit, i.e. $N=10$. Residuals were checked for normality, and frequency data were square root transformed if needed. Mixed models with housing system and batch as fixed effects were used for the following variables: the number of live born, stillborn, dead, cross-fostered, and weaned piglets; weaning age; feed intake; weight gain; the number of days piglets suffered from pasty or watery faeces; the number of days piglets were treated with antibiotics or analgesics; and damage 
scores (excluding skin). Behaviour, skin lesions, average faecal consistency score (calculated as: normal faeces $=0$, pasty faeces $=1$, and watery faeces $=$ 2), and blood parameters were analysed with mixed models including housing system, day of measurement, and housing $\mathrm{x}$ day interaction. Repeated observations on the same group of animals were taken into account by including a repeated effect of housing system within batch. Significant interaction effects were further analysed using post hoc tests. In case of multiple pairwise comparisons for skin lesions and post-weaning behavioural data, a Tukey adjustment was used. Effects of housing system on proportion of treated animals, proportion of non-eaters, and proportion of piglets with pasty or watery faeces were analysed with generalised linear mixed models using a binomial distribution and logit link function, and including batch as fixed effect. Results are presented as means \pm SEM (based on averages per system per batch). $P$ - values below 0.05 are considered statistically significant.

\subsection{Results}

\subsubsection{Piglet mortality}

Pre-weaning mortality was higher among ML litters than among SL litters (3.22 \pm 0.42 vs. $1.52 \pm 0.25$ piglets per litter, $P<0.01)$, specifically due to crushing ( $2.34 \pm 0.44$ vs. $0.20 \pm 0.09, P<0.01)$. Mortality due to other causes was lower in the ML system than in the SL system $(0.88 \pm 0.27$ vs. $1.32 \pm 0.20$, $P<0.05$ ). Mortality mostly occurred before grouping of ML litters (ML: $2.68 \pm$ 0.42 , SL: $1.32 \pm 0.21, P<0.01)$. Litter sizes on the day of ML litter grouping did not differ (ML: $12.19 \pm 0.32$, SL: $12.52 \pm 0.21$ ). Mortality after grouping of ML litters was $0.54 \pm 0.07$ in the ML system and $0.20 \pm 0.15$ in the SL system $(P=0.07)$, resulting in a litter size at weaning of $11.65 \pm 0.27$ in the ML system and $12.32 \pm 0.22$ in the SL system $(P=0.06)$. When only considering the litters that were selected for further post-weaning investigation, litter sizes at weaning were $11.85 \pm 0.32$ in the ML system and $12.60 \pm 0.17$ in the SL system $(P=$ 0.08). After weaning, $2 \mathrm{ML}$ piglets died (1 of streptococcal infection and 1 of unknown causes) and 3 SL piglets died (1 of streptococcal infection and 2 of unknown causes). 


\subsubsection{Feed intake and body weight}

ML piglets showed a higher frequency of feed-directed behaviour on day 13 before weaning than SL piglets $(6.80 \pm 0.96$ vs. $2.35 \pm 0.59)$, whereas frequencies on day 2 before weaning did not differ (housing system $x$ day interaction, $P<0.05$; Figure 3.2). When distinguishing between the types of feed the behaviour was directed at, it was observed that ML piglets were more frequently in contact with sow feed than SL piglets on both observation days combined ( $4.06 \pm 0.33$ vs. $0.11 \pm 0.03$ occurrences per hour, $P<0.001$; Figure 3.2). SL piglets, however, showed an increasing interest in piglet feed over time (housing system $\mathrm{x}$ day interaction, $P<0.001$ ); there was no difference in contact with piglet feed between housing systems on day -13 (ML: $3.04 \pm 0.56$, SL: $2.21 \pm 0.62)$, but on day -2 SL piglets were more frequently in contact with piglet feed than ML piglets ( $6.56 \pm 1.59$ vs. $2.40 \pm 0.44$ occurrences per hour). The frequency of drinking behaviour (ML: $0.61 \pm 0.11$, SL: $1.07 \pm 0.15$ ) and the percentage of non-eaters based on faecal sampling on the day before weaning (ML: $29.7 \pm 5.6 \%$, SL: $36.4 \pm 5.5 \%$ ) did not differ between housing systems.

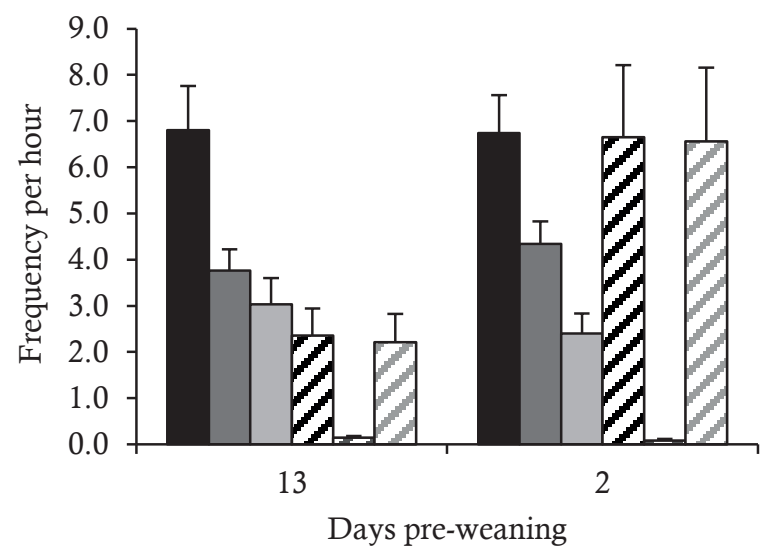

Figure 3.2. Pre-weaning feed-directed behaviour (consisting of sniffing, nosing, and eating feed) of piglets in a multi-litter system (solid) and piglets in a single-litter system (dashed). Black $=$ behaviour directed at sow and piglet feed (housing system $\mathrm{x}$ day interaction, $P<$ 0.05); dark grey $=$ behaviour directed at sow feed (housing effect, $P<0.001$ ); light grey = behaviour directed at piglet feed (housing system $\mathrm{x}$ day interaction, $P<0.001$ ). 
Weaning weight (day -1) did not differ between housing systems, neither before (ML: $8.21 \pm 0.21 \mathrm{~kg}$, SL: $8.25 \pm 0.15 \mathrm{~kg}$ ) nor after (ML: $8.37 \pm 0.21 \mathrm{~kg}, \mathrm{SL}$ : $8.30 \pm 0.18 \mathrm{~kg}$ ) selecting piglets for further post-weaning investigation. The SD of weaning weights also did not differ between systems before and after selection (data not shown). After weaning, ML piglets had a higher weight gain than SL piglets between days -1 and 2, days 2 and 5, days 13 and 35, and days -1 and 35 (Table 3.1). Feed intake was higher for ML piglets between days 1 and 2 post-weaning only (Table 3.1). Feed efficiency from weaning until 9 weeks of age did not differ between housing systems (ML: $0.69 \pm 0.01$, SL: $0.68 \pm 0.01)$.

Table 3.1. Post-weaning feed intake and weight gain of piglets raised in a multi-litter system and piglets raised in a single-litter system.

\begin{tabular}{lcc}
\hline & \multicolumn{2}{c}{ Housing $^{1}$} \\
\cline { 2 - 3 } Variable & \multicolumn{1}{c}{$\mathrm{ML}$} \\
\hline Intake per piglet, $k g$ & & \\
\hline Days 0 to 1 & $0.15 \pm 0.03$ & $0.14 \pm 0.01$ \\
Days 1 to 2 & $0.29 \pm 0.02$ & $0.16 \pm 0.03^{* *}$ \\
Days 2 to 5 & $0.77 \pm 0.15$ & $0.68 \pm 0.09$ \\
Days 5 to 13 & $3.47 \pm 0.31$ & $2.48 \pm 0.28$ \\
Days 13 to 35 & $19.78 \pm 1.33$ & $16.71 \pm 0.25$ \\
Days 0 to 35 & $24.46 \pm 1.57$ & $20.18 \pm 0.43$ \\
Weight gain per piglet, $k g$ & & \\
Days -1 to 2 & $0.59 \pm 0.06$ & $0.35 \pm 0.03^{* *}$ \\
Days 2 to 5 & $0.76 \pm 0.17$ & $0.39 \pm 0.14^{*}$ \\
Days 5 to 13 & $2.5 \pm 0.2$ & $1.8 \pm 0.1$ \\
Days 13 to 35 & $13.2 \pm 0.9$ & $11.1 \pm 0.3^{*}$ \\
Days -1 to 35 & $17.0 \pm 1.2$ & $13.7 \pm 0.4^{*}$ \\
\hline${ }^{1} \mathrm{ML}=$ multi-litter housing, SL $=$ single-litter housing. \\
* $P<0.05, * * P<0.01$.
\end{tabular}

\subsubsection{Play, damaging oral manipulation, aggression, and belly-nosing}

Before weaning, the occurrence of play behaviour did not differ between housing systems (ML: $5.6 \pm 0.4$, SL: $3.9 \pm 0.7$ occurrences per hour; Figure 3.3A). When looking at different elements of play behaviour, however, ML piglets showed a higher frequency of individual play $(2.3 \pm 0.2$ vs. $1.5 \pm 0.2$ occurrences per hour, $P<0.05)$. Occurrence of social play and substrate play did not differ (data not shown). Furthermore, ML piglets showed a lower 
frequency of damaging oral manipulation before weaning (1.4 \pm 0.2 vs. $3.6 \pm$ 0.3 occurrences per hour, $P<0.001$; Figure $3.3 \mathrm{C}$ ). Analysis per behavioural element within the category of manipulation revealed that ML piglets manipulated sows $(P<0.05)$ and piglets' tails $(P<0.01)$, ears $(P<0.01)$, and other body parts $(P<0.01)$ less often than SL piglets (data not shown). The occurrence of aggressive behaviour did not differ between housing systems (Figure 3.3E).

Averaged over the entire post-weaning period, ML piglets played more often than SL piglets $(4.0 \pm 0.3$ vs. $2.8 \pm 0.3$ occurrences per hour, $P<0.05$; Figure $3.3 \mathrm{~B})$ and specifically showed more substrate play $(2.5 \pm 0.2$ vs. $1.5 \pm 0.2$ occurrences per hour, $P<0.05)$. There was no difference in occurrence of individual and social play (data not shown). The occurrence of damaging oral manipulation (Figure 3.3D) was lower in ML piglets than in SL piglets (1.8 \pm 0.3 vs. $3.5 \pm 0.4$ occurrences per hour, $P<0.01)$ and increased over time $(P<$ 0.0001 ), but sooner after weaning in SL piglets than in ML piglets (housing system $\mathrm{x}$ day interaction, $P<0.01)$. This interaction held specifically for manipulation of ears $(P<0.05)$ and tails $(P<0.001)$. The overall occurrence of aggression varied over time (day effect $P<0.05$; Figure 3.3F), but did not differ between housing systems. ML piglets, however, showed more biting behaviour $(1.04 \pm 0.07$ vs. $0.65 \pm 0.05$ occurrences per hour, $P<0.05)$ and less aggression near the feeder $(0.34 \pm 0.04$ vs. $0.92 \pm 0.10$ occurrences per hour, $P<0.01)$. There was no difference in the occurrence of head knocking and fighting (data not shown). Lastly, ML piglets showed a lower peak in belly-nosing frequency after weaning than SL piglets (housing system $\mathrm{x}$ day interaction, $P<0.05$; Figure 3.3G).

\subsubsection{Damage scores}

ML piglets had a higher average snout damage score on the day before weaning than SL piglets (Table 3.2). The damage scores for the front paws (around the carpal joints), ears, and tail did not differ between housing systems on the day before weaning and on day 33 after weaning (Table 3.2). There was an interaction between housing system and day regarding the number of skin lesions (Figure 3.4). There was no difference in the total number of lesions between housing systems on the day before weaning, but ML piglets had more deep lesions than SL piglets $(0.42 \pm 0.06$ vs. $0.21 \pm 0.04, P<0.05)$. The post- 


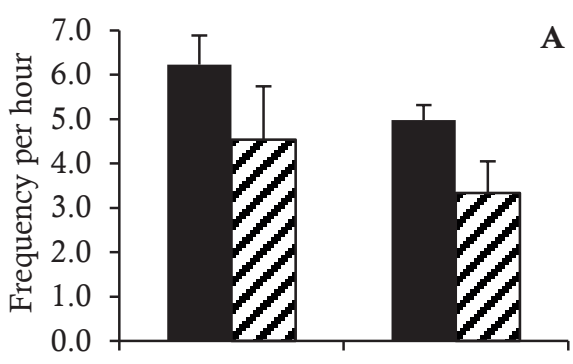

13

A

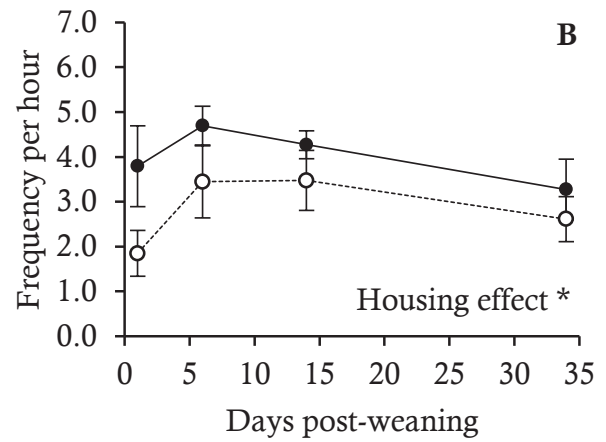

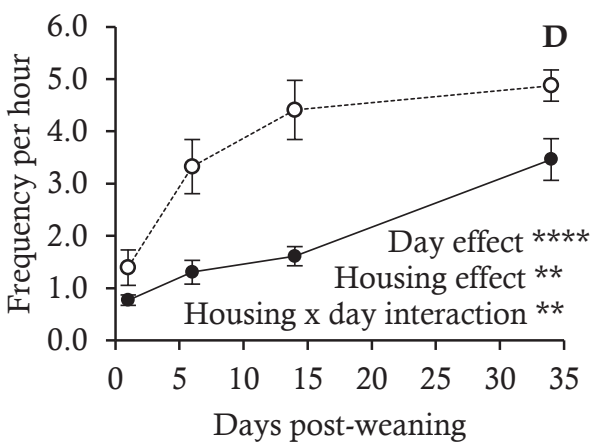
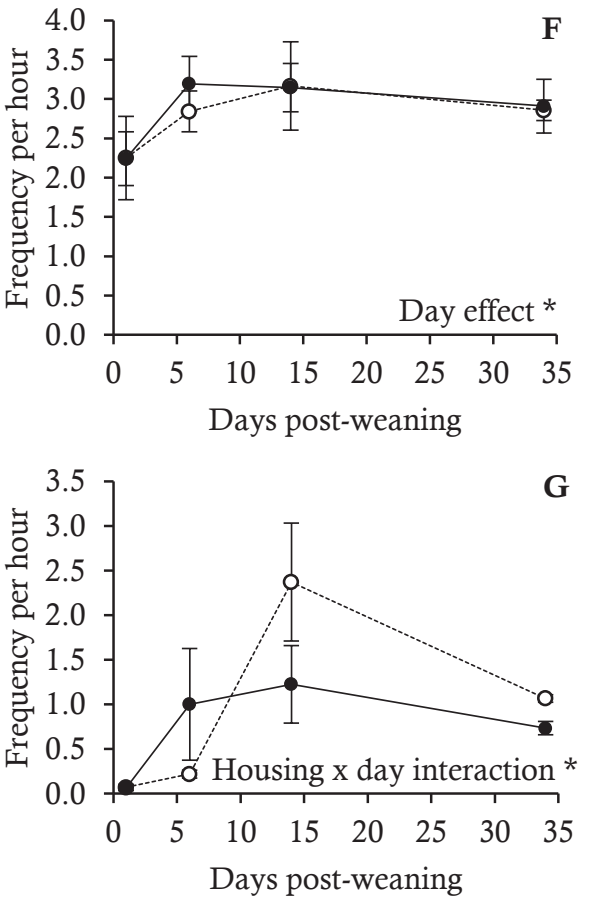

Figure 3.3. Behaviour of piglets raised in a multi-litter system (solid) and piglets raised in a single-litter system (dashed). Pre-weaning (A) and post-weaning (B) play behaviour consists of individual, social, and substrate play. (continued on the next page) 
weaning increase in the number of skin lesions on day 2 and day 5 after weaning was lower for ML piglets than for SL piglets. ML piglets also had fewer deep lesions than SL piglets on day $5(1.33 \pm 0.53$ vs. $2.64 \pm 0.77, P<$ 0.05). For ML piglets, the number of lesions was similar on days 2, 5, and 33 . SL piglets, however, showed a post-weaning decrease in the number of lesions, resulting in fewer lesions on day 33 compared with $\mathrm{ML}$ piglets. ML piglets also had more deep lesions on day $33(0.76 \pm 0.16$ vs. $0.41 \pm 0.08, P<0.05)$.

Table 3.2. Scores for damage on snout, front paws, ears, and tail relative to the day of weaning (day 0) for piglets raised in a multi-litter (ML) system and piglets raised in a single-litter (SL) system.

\begin{tabular}{lccccc}
\hline \multirow{2}{*}{ Variable } & \multicolumn{2}{c}{ Day -1} & & \multicolumn{2}{c}{ Day 33 } \\
\cline { 2 - 3 } \cline { 5 - 6 } & ML & SL & & ML & SL \\
\hline Snout damage score & $1.55 \pm 0.07$ & $1.22 \pm 0.04^{*}$ & & - & - \\
Paw damage score $^{1}$ & $0.77 \pm 0.11$ & $1.08 \pm 0.13$ & & $0.07 \pm 0.02$ & $0.05 \pm 0.03$ \\
Ear damage score $^{2}$ & $1.06 \pm 0.13$ & $0.87 \pm 0.17$ & & $1.70 \pm 0.35$ & $1.36 \pm 0.09$ \\
Tail damage score & $0.88 \pm 0.20$ & $0.79 \pm 0.09$ & & $1.13 \pm 0.14$ & $1.27 \pm 0.08$ \\
\hline
\end{tabular}

\footnotetext{
${ }^{1}$ Score averaged for both paws.

${ }^{2}$ Score averaged for both ears.

${ }^{*} P<0.05$.
}

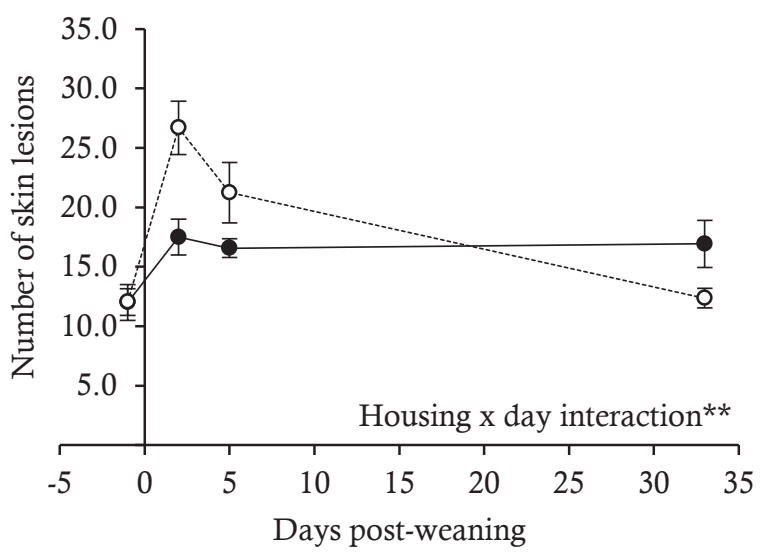

Figure 3.4. The total number of fresh skin lesions (scratches) per piglet for piglets raised in a multi-litter system (solid) and piglets raised in a single-litter system (dashed), ** $P<0.01$.

Figure 3.3. (continued). Pre-weaning (C) and post-weaning (D) damaging oral manipulation consists of chewing on a body part (e.g. tail or ear) of another pig. Pre-weaning (E) and postweaning (F) aggressive behaviour consists of head knocking, biting, and fighting. Graph $\mathrm{G}$ represents the post-weaning occurrence of belly-nosing. ${ }^{*} P<0.05$, ** $P<0.01$, *** $P<0.001$, **** $P<0.0001$. 


\subsubsection{Health and blood parameters}

Housing system did not affect the overall percentage of piglets with at least 1 day of pasty or watery faeces (ML: $84.1 \pm 10.2 \%$, SL: $93.9 \pm 3.3 \%$ ) and the number of days piglets suffered from pasty or watery faeces between days 0 and 14 post-weaning (ML: $3.53 \pm 0.97$, SL: $4.63 \pm 0.57$; Figure 3.5). The average faecal consistency score was, however, lower (i.e. more solid faeces) for ML piglets than for SL piglets $(0.27 \pm 0.03$ vs. $0.39 \pm 0.03, P<0.05)$ and varied over time $(P<0.0001)$, showing a peak around day 5 (Figure 3.5 ).

KLH-IgM titres increased over time $(P<0.0001)$ and were higher on the day of weaning in $\mathrm{ML}$ piglets than in SL piglets $(2.16 \pm 0.13$ vs. $1.55 \pm 0.12$, housing system $\mathrm{x}$ day interaction, $P<0.05$; Figure 3.6A). KLH-IgG titres were unaffected by sampling day and housing system (Figure 3.6B). Haptoglobin and leukocyte concentrations did not differ between housing systems, but did show an increase between days 0 and 13 post-weaning, and remained similar between days 13 and 35 (Figure 3.6C, 3.6D). The percentage of piglets injected with antibiotics or analgesics did not differ between housing systems, neither before weaning (ML: $7.4 \pm 1.4 \%$, SL: $1.8 \pm 0.6 \%$ ) nor after weaning (ML: 7.0 $\pm 2.4 \%$, SL: $9.5 \pm 2.4 \%$ ). Piglets were mainly treated for symptoms of lameness and, after weaning, also for streptococcal infection. It should be noted that in 1

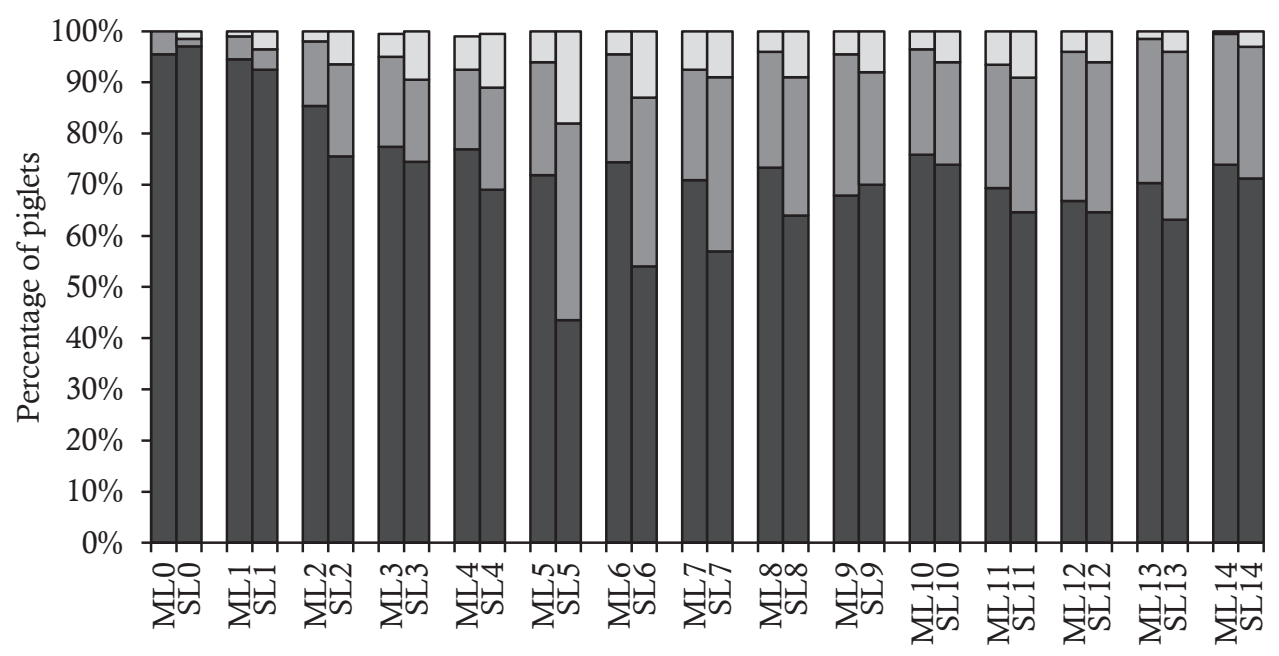

Figure 3.5. Faecal consistency scores from days 0 to 14 post-weaning for piglets raised in a multi-litter system (ML) and piglets raised in a single-litter system (SL). Scores were based on visual assessment of the backside of each piglet, containing either no faeces ( $\square$ ), pasty faeces $(\varpi)$, or watery faeces $(\square)$. 
batch, all ML piglets were additionally treated after weaning (via the feed during 6 days) to prevent the spread of streptococcal infection.
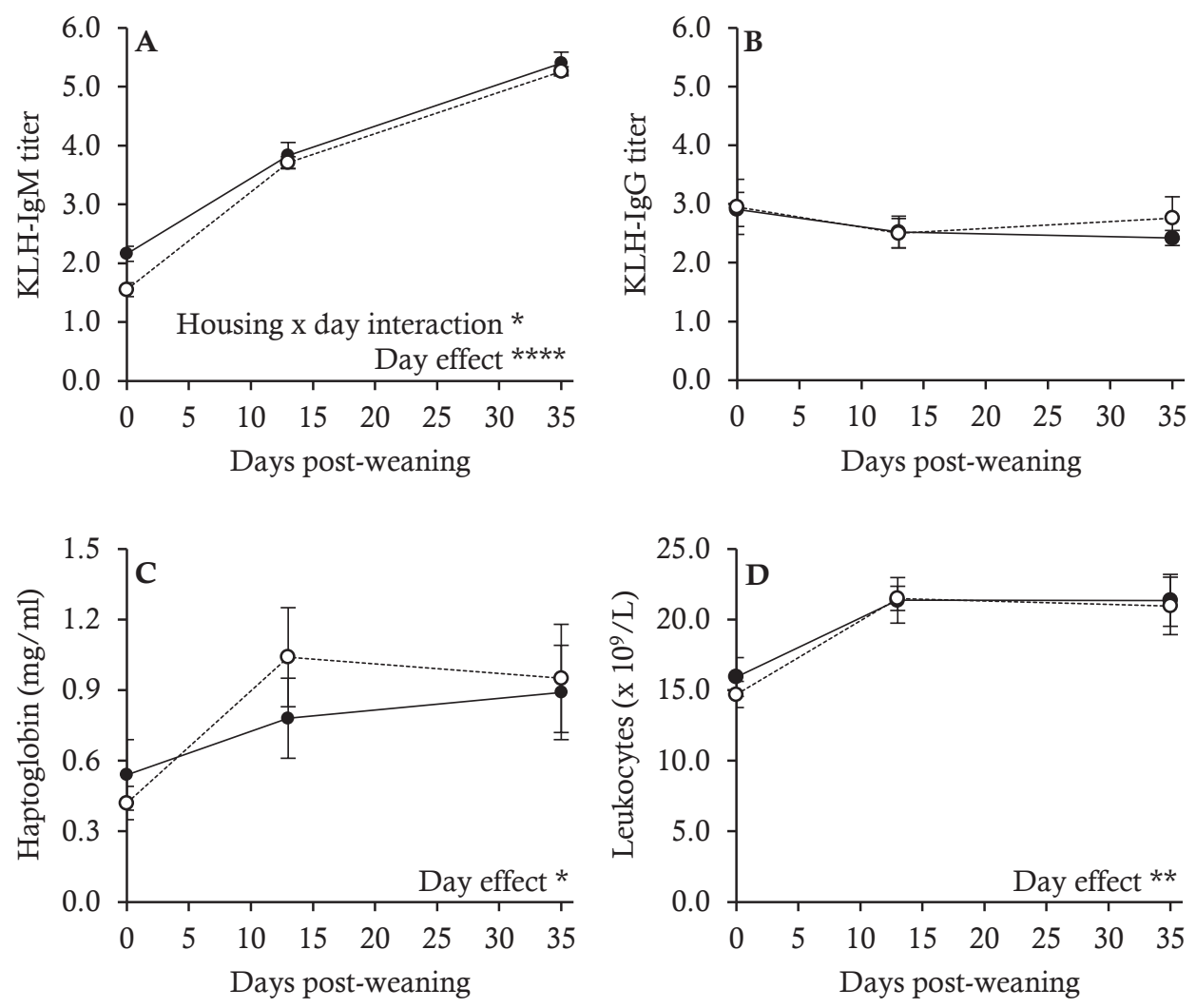

Figure 3.6. Post-weaning KLH-IgM titers (A), KLH-IgG titers (B), plasma haptoglobin concentrations (C), and leukocyte concentrations (D) of piglets raised in a multi-litter system (solid) and piglets raised in a single-litter system (dashed).

* $P<0.05$, ** $P<0.01$, **** $P<0.0001$.

\subsection{Discussion}

This study compared the development of piglets raised in either a multi-litter (ML) system or a single-litter (SL) system until 9 weeks of age. ML piglets showed more feed-directed behaviour during early lactation, had a higher feed intake in the early post-weaning period, had a more solid faecal consistency after weaning, showed a $24 \%$ higher post-weaning weight gain until 9 weeks of age, and displayed more play behaviour and less damaging oral manipulation of other pigs like tail biting and ear biting. 


\subsubsection{Pre-weaning development}

Pre-weaning mortality was higher in the ML system, mainly as a result of crushing in the first week post-partum, possibly related to the loose farrowing pens in the ML system. We cannot fully exclude the possibility that the enhanced performance of ML piglets was partly the result of a higher selection pressure for more vital piglets. Birth weight of piglets that died was, however, higher in the ML system than in the SL system $(1.33 \pm 0.03$ vs. $1.00 \pm 0.06, P$ $<0.05$ ) and housing effects on post-weaning weight gain were still significant when excluding litters in which 3 or more piglets died. Moreover, as a result of cross-fostering, litter sizes at grouping $(P>0.10)$ and at weaning $(0.05<P<$ $0.10)$ were similar in both systems and weaning weights also did not differ.

ML piglets showed almost a threefold higher frequency of feed-directed behaviour around 2 weeks of age, suggesting that they started exploring and ingesting feed earlier than SL piglets. Social facilitation of eating behaviour and social learning from the floor-fed sows and other piglets (Nicol and Pope, 1994; Morgan et al., 2001; Oostindjer et al., 2011a) may have stimulated early foraging in ML piglets, whereas SL piglets had limited possibilities to observe or participate in the sow's eating behaviour. The interest of ML piglets in the sows' feed, besides by having easier access, could be mediated by exposure to the flavours from the sows' diet in utero and via milk (Langendijk et al., 2007; reviewed by Bolhuis et al., 2009; Oostindjer et al., 2009; also see Figueroa et al., 2013b). The more enriched environment in the multi-suckling system may also have facilitated early eating behaviour, as more chewing material was available. Substrates, like straw, can stimulate the development of foragingrelated behaviours (reviewed by Oostindjer et al., 2014). The frequency of eating behaviour mainly seemed to differ in the first part of lactation, suggesting that ML piglets started to eat earlier than SL piglets. Perhaps this positively affects composition of the gut microbiota, with potential beneficial effects on intestinal health (Rist et al., 2012). There was no difference between systems in frequency of feed-directed behaviour 2 days before weaning and in the percentage of piglets classified as non-eaters on the day before weaning, although we have no data on the actual feed intake before weaning.

Furthermore, ML piglets showed a lower frequency of damaging oral manipulation towards other pigs, such as tail and ear biting. This can be related 
to the more enriched environment that was available to the ML piglets and the lower pre-weaning stocking density (reviewed by Taylor et al., 2010). The lower frequency of manipulation was, however, not reflected in lower ear and tail damage scores. This could indicate that on the relatively few occasions that ML piglets showed manipulation, this occurred more intensely compared with SL piglets. Additionally, in the larger ML group with more potential victims, a single manipulating piglet could cause more damage than a single piglet in an SL pen.

The frequency of aggressive behaviour and the number of skin lesions related to aggression did not differ between housing systems before weaning. Similarly, Kutzer et al. (2009) found no difference in skin lesion scores on day 14 of lactation between litters that had contact with other litters from day 10 onward and unmixed litters. ML piglets did, however, have more deep skin lesions than SL piglets. Additionally, ML piglets had a higher average snout damage score on the day before weaning, probably reflecting increased competition at the udder, associated with the occurrence of cross-suckling (reviewed by Van Nieuwamerongen et al., 2014). Lastly, it should be noted that (although not significantly different) the percentage of piglets that received veterinary treatment before weaning was about 4 times higher in the ML group than in the SL group, which was mainly due to lameness. This was likely related to the more abrasive concrete flooring covering a large part of the multisuckling system (used to ensure a good grip for the sows) which could inflict injuries with risk of infection. The higher occurrence of veterinary treatment for lameness was, however, not reflected in a higher paw damage score.

Furthermore, naturally occurring antibodies (Nabs) binding KLH were measured. Nabs are likely part of innate immunity and form an important first line of defence against pathogens (Lutz, 2012). ML piglets had higher titres of IgM binding KLH compared with SL piglets on the day of weaning. It can be speculated that this reflects an accelerated immune development, which may be driven by early contact with solid feed and/or enrichment materials that act as antigenic stimuli. The gut is an important site of antibody production in the pig (Bianchi et al., 1999), and dietary factors can influence IgM levels in piglets (Molist et al., 2014). 
In conclusion, the different pre-weaning environment affected (behavioural) development, which may have resulted in more mature piglets at weaning in the ML system compared with the SL system.

\subsubsection{Performance in the early post-weaning period}

ML piglets ate $81 \%$ more feed between days 1 and 2 after weaning and had an $82 \%$ higher weight gain until day 5 after weaning than SL piglets. Within the first 2 weeks after weaning, ML piglets showed a lower peak in belly-nosing and a more solid faecal consistency, the latter indicating a lower occurrence of diarrhoea. Thus, the ML piglets seemed to have less difficulty making the transition to a fully solid diet at weaning than the SL piglets. The earlier onset of pre-weaning sampling of solid feed may have contributed to the higher postweaning feed intake of ML piglets (see Van den Brand et al., 2014). In addition, Oostindjer et al. (2010) found that feed intake in the first 2 days postweaning was affected by pre-weaning enrichment, but not by post-weaning enrichment. Moreover, feed intake in the first 2 weeks after weaning was unaffected by pre- and post-weaning enrichment. This may suggest that early contact with enrichment materials (that can stimulate development of foragingrelated behaviours) before weaning is important for the early post-weaning transition to solid feed intake, whereas post-weaning physical enrichment seems less important in this regard. The post-weaning social environment may, however, have influenced feed intake because ML pens contained in total more experienced eaters than SL pens, with a similar percentage of eaters at weaning. As inexperienced eaters can benefit from being housed with experienced eaters (Morgan et al., 2001; Figueroa et al., 2013a), non-eater ML piglets may have been more stimulated to start eating after weaning than noneater SL piglets. Correspondingly, the lower peak in belly-nosing seems to reflect the ML piglets' more advanced level of ingestive development and better transition from a milk-based diet to a diet of only solid feed (reviewed by Widowski et al., 2008).

One day after weaning, there seemed to be a decrease in play behaviour compared with pre-weaning levels on day -2 , especially in the SL piglets. This may reflect a temporary decrease in welfare associated with weaning (Donaldson et al., 2002) as play levels on day 6 seemed to be restored to frequencies observed on day -2 . One weaning-related stressor that the ML 
piglets may have been better able to cope with is the exposure to a new environment. Piglets raised in a multi-suckling system have been reported to adapt better to novel situations than piglets raised with individually housed sows, as indicated by more time spent exploring a new environment, fewer escape attempts, and a lower occurrence of high-frequency vocalisations (Hillmann et al., 2003).

The intensity of aggressive encounters seemed to be lower for ML piglets, as they had fewer (deep) lesions than SL piglets on days 2 and 5 post-weaning. This may reflect a better response of the ML piglets to the change in social structure after weaning (i.e. smaller group size and accompanying rearrangement of dominance ranks). D'Eath (2005) found that piglets that had gained access to a neighbouring farrowing crate from day 10 of lactation onward (compared with piglets housed as 1 litter), formed a stable social hierarchy more quickly when confronted with unfamiliar piglets on day 20 after weaning. Possibly, this improved social development is not only displayed when encountering unfamiliar piglets, but also when the social structure changes within a group of piglets that are already familiar with each other. The lower number of lesions in ML piglets may also reflect less frustrationassociated aggression (see Dantzer et al., 1980), related to the better transition to the new situation after weaning, compared with SL piglets. Additionally, SL piglets could have nasal contact with unfamiliar piglets in adjacent pens through partially bar-fenced pen partitions. Piglets from adjacent pens could not fight freely, which may have elicited redirected aggression towards penmates. Lastly, it should be noted that unfamiliar piglets are often mixed at weaning in commercial practice, which generally results in fighting and skin lesions. Piglets raised in an ML system likely show less intense aggression than piglets raised in an SL system toward unfamiliar piglets at weaning (see Hillmann et al., 2003; Li and Wang, 2011), although post-weaning mixing may be unnecessary altogether if an ML system is used.

In sum, a combination of the difference in pre-weaning development and postweaning environment likely resulted in a better transition from the pre-weaning to the post-weaning situation for the ML piglets compared with the SL piglets. 


\subsubsection{Post-weaning performance until 9 weeks of age}

Over the entire post-weaning phase, ML piglets showed a higher frequency of play behaviour and a lower frequency of damaging oral manipulation than SL piglets. Similar to the situation before weaning, oral manipulation of pen-mates may have been limited by the availability of rooting and chewing materials (Bolhuis et al., 2005) in the ML system, whereas the larger total area and availability of enrichment materials may have stimulated play behaviour (Chaloupková et al., 2007). The behavioural differences between ML and SL piglets may, however, also reflect the improved transition of the ML piglets to the post-weaning phase. Several strategies that can facilitate the transition after weaning, such as learning from the sow (Oostindjer et al., 2011c) and prenatal flavour learning (Oostindjer et al., 2011b), resulted in reduced oral manipulation of pen-mates and increased play behaviour, up to at least 2 weeks post-weaning. Play behaviour is associated with positive welfare as it is typically shown under favourable conditions and is suppressed under detrimental circumstances (Held and Špinka, 2011).

It is remarkable that when comparing days 14 and 34 after weaning, the frequency of manipulation stayed relatively constant in SL housing, whereas the ML piglets doubled the frequency of this behaviour. Possibly, this is because ML piglets started to lose interest in the provided enrichment material. Yet, the frequency of substrate play did not decrease over time, so perhaps a more pertinent explanation is the reduction in space availability. The ML and SL group had an equal space allowance per piglet, but as ML piglets grew faster, they occupied more space near the end of the post-weaning period. Crowding -especially when piglets begin to 'fill their pen'- can elicit tail biting behaviour (Taylor et al., 2010). Additionally, ML piglets may have experienced the crowding effect more intensely than SL piglets as ML piglets had a smaller total area available in their post-weaning pen compared with their pre-weaning pen, whereas SL piglets did not experience a change in pen size. The higher skin lesion scores of $\mathrm{ML}$ piglets on day 33 may also be a reflection of frustration due to crowding, although the frequency of aggressive encounters did not differ between the 2 housing systems.

The $24 \%$ higher weight gain of ML piglets compared with SL piglets over the entire post-weaning period, despite similar weaning weights, is likely related to 
the better transition around weaning, resulting in an improved feed intake and perhaps enhanced gut development. A number of studies have suggested an impact of early post-weaning performance on long-term growth (Tokach et al., 1992; Campbell et al., 2013). Also the lower occurrence of manipulation may have contributed to a higher growth rate (see Camerlink et al., 2012).

In short, the improved post-weaning performance of $\mathrm{ML}$ piglets compared with SL piglets is likely explained by a combination of the difference in pre-weaning development, early post-weaning performance, and post-weaning environment.

Although it is not possible to fully disentangle the contributing aspects of the social and physical environment, overall, ML housing seems to provide many benefits for piglet development.

\subsection{Implications}

Piglets raised in a multi-suckling system, followed by post-weaning housing in a group of 40 in an enriched pen, showed a better pre-weaning behavioural development, an improved transition to the post-weaning phase, and a better post-weaning performance, compared with piglets raised in a standard pen with only litter-mates. This was mainly indicated by an early interest in solid feed before weaning, a higher feed intake in the early post-weaning period, a more solid faecal consistency, a higher weight gain, a higher frequency of play behaviour, and a lower frequency of damaging oral manipulation, like tail biting. Some challenges that remain in the multi-suckling system are a higher pre-weaning mortality, mainly due to crushing in the loose farrowing pens before grouping, and a higher occurrence of lameness, likely related to the more abrasive flooring. Additionally, at the end of the post-weaning phase, there were some indicators of frustration in the ML system, possibly related to crowding. Overall, provided that pre-weaning mortality can be reduced, the multi-litter system from 0 to 9 weeks of age seems promising for raising robust piglets with better welfare.

\subsection{Acknowledgements}

The authors gratefully acknowledge the Ministry of Economic Affairs for their financial support and the staff of Swine Innovation Centre Sterksel for their assistance. Furthermore, the authors want to thank Linda Troquet, Bjorge 
Laurenssen, Jan Wijnen, Monique Ooms, Fleur Bartels, Mike Nieuwland, Ger de Vries-Reilingh, Miranda Elling-Staats, and Peggy Friesen for technical assistance and help with collecting and/or analysing data. Lastly, Henk Parmentier is acknowledged for providing valuable feedback on the manuscript. 


\subsection{References}

Bianchi A.T.J., Scholten J.W., Moonen Leusen B.H.W.M., and Boersma W.J.A. 1999. Development of the natural response of immunoglobulin secreting cells in the pig as a function of organ, age and housing. Developmental and Comparative Immunology 23: 511-520.

Bohnenkamp A.L., Meyer C., Müller K., and Krieter J. 2013. Group housing with electronically controlled crates for lactating sows. Effect on farrowing, suckling and activity behavior of sows and piglets. Applied Animal Behaviour Science 145: 37-43.

Bolhuis J.E., Oostindjer M., Van den Brand H., Gerrits W.J.J., and Kemp B. 2009. Voluntary feed intake in piglets: Potential impact of early experience with flavours derived from the maternal diet. In: Torrallardona D. and Roura E. (eds.) Voluntary Feed Intake in Pigs. p 37-60. Wageningen Academic Publishers, The Netherlands.

Bolhuis J.E., Schouten W.G.P., Schrama J.W., and Wiegant V.M. 2005. Behavioural development of pigs with different coping characteristics in barren and substrate-enriched housing conditions. Applied Animal Behaviour Science 93: 213-228.

Camerlink I., Bijma P., Kemp B., and Bolhuis J.E. 2012. Relationship between growth rate and oral manipulation, social nosing, and aggression in finishing pigs. Applied Animal Behaviour Science 142: 11-17.

Campbell J.M., Crenshaw J.D., and Polo J. 2013. The biological stress of early weaned piglets. Journal of Animal Science and Biotechnology 4.

Chaloupková H., Illmann G., Bartoš L., and Špinka M. 2007. The effect of preweaning housing on the play and agonistic behaviour of domestic pigs. Applied Animal Behaviour Science 103: 25-34.

D'Eath R.B. 2005. Socialising piglets before weaning improves social hierarchy formation when pigs are mixed post-weaning. Applied Animal Behaviour Science 93: 199-211.

Dantzer R., Arnone M., and Mormede P. 1980. Effects of frustration on behaviour and plasma corticosteroid levels in pigs. Physiology and Behavior 24: 1-4. 
Donaldson T.M., Newberry R.C., Špinka M., and Cloutier S. 2002. Effects of early play experience on play behaviour of piglets after weaning. Applied Animal Behaviour Science 79: 221-231.

Figueroa J., Solà-Oriol D., Manteca X., and Pérez J.F. 2013a. Social learning of feeding behaviour in pigs: Effects of neophobia and familiarity with the demonstrator conspecific. Applied Animal Behaviour Science 148: 120-127.

Figueroa J., Solà-Oriol D., Vinokurovas L., Manteca X., and Pérez J.F. $2013 b$. Prenatal flavour exposure through maternal diets influences flavour preference in piglets before and after weaning. Animal Feed Science and Technology 183: 160-167.

Held S.D.E. and Špinka M. 2011. Animal play and animal welfare. Animal Behaviour 81: 891-899.

Hillmann E., Von Hollen F., Bünger B., Todt D., and Schrader L. 2003. Farrowing conditions affect the reactions of piglets towards novel environment and social confrontation at weaning. Applied Animal Behaviour Science 81: 99-109.

Kutzer T., Bünger B., Kjaer J.B., and Schrader L. 2009. Effects of early contact between non-littermate piglets and of the complexity of farrowing conditions on social behaviour and weight gain. Applied Animal Behaviour Science 121: $16-24$.

Langendijk P., Bolhuis J.E., and Laurenssen B.F.A. 2007. Effects of pre- and postnatal exposure to garlic and aniseed flavour on pre- and postweaning feed intake in pigs. Livestock Science 108: 284-287.

Li Y. and Wang L. 2011. Effects of previous housing system on agonistic behaviors of growing pigs at mixing. Applied Animal Behaviour Science 132: 20-26.

Lutz H.U. 2012. Naturally Occurring Antibodies (NAbs). Springer Science+Business Media.

Molist F., van Eerden E., Parmentier H.K., and Vuorenmaa J. 2014. Effects of inclusion of hydrolyzed yeast on the immune response and performance of piglets after weaning. Animal Feed Science and Technology 195: 136-141.

Morgan C.A., Lawrence A.B., Chirnside J., and Deans L.A. 2001. Can information about solid food be transmitted from one piglet to another? Animal Science 73: 471-478. 
Nicol C.J. and Pope S.J. 1994. Social learning in sibling pigs. Applied Animal Behaviour Science 40: 31-43.

Oostindjer M., Bolhuis J.E., Mendl M., Held S., Gerrits W., Van den Brand H., and Kemp B. 2010. Effects of environmental enrichment and loose housing of lactating sows on piglet performance before and after weaning. Journal of Animal Science 88: 3554-3562.

Oostindjer M., Bolhuis J.E., Mendl M., Held S., van den Brand H., and Kemp B. 2011a. Learning how to eat like a pig: Effectiveness of mechanisms for vertical social learning in piglets. Animal Behaviour 82: 503-511.

Oostindjer M., Bolhuis J.E., Simon K., van den Brand H., and Kemp B. 2011b. Perinatal flavour learning and adaptation to being weaned: All the pig needs is smell. PLoS ONE 6.

Oostindjer M., Bolhuis J.E., Van den Brand H., and Kemp B. 2009. Prenatal flavor exposure affects flavor recognition and stress-related behavior of piglets. Chemical Senses 34: 775-787.

Oostindjer M., Kemp B., van den Brand H., and Bolhuis J.E. 2014. Facilitating 'learning from mom how to eat like a pig' to improve welfare of piglets around weaning. Applied Animal Behaviour Science 160: 19-30.

Oostindjer M., van den Brand H., Kemp B., and Bolhuis J.E. 2011c. Effects of environmental enrichment and loose housing of lactating sows on piglet behaviour before and after weaning. Applied Animal Behaviour Science 134: $31-41$.

Phillips C.E., Farmer C., Anderson J.E., Johnston L.J., Shurson G.C., Deen J., Keisler D.H., Conner A.M., and Li Y.Z. 2014. Preweaning mortality in group-housed lactating sows: Hormonal differences between high risk and low risk sows. Journal of Animal Science 92: 2603-2611.

Reimert I., Rodenburg T.B., Ursinus W.W., Kemp B., and Bolhuis J.E. 2014. Selection based on indirect genetic effects for growth, environmental enrichment and coping style affect the immune status of pigs. PLoS ONE 9: e108700. 
Rist V.T.S., Eklund M., Bauer E., Sauer N., and Mosenthin R. 2012. Effect of feeding level on the composition of the intestinal microbiota in weaned piglets. Journal of Animal Science 90: 19-21.

Schau D.J., Brue J.D., and Rosentrater K.A. 2013. Review of housing options for gestating sows. In: American Society of Agricultural and Biological Engineers Annual International Meeting 2013, ASABE 2013. p 1542-1567.

Taylor N.R., Main D.C., Mendl M., and Edwards S.A. 2010. Tail-biting: a new perspective. The Veterinary Journal 186: 137-147.

Tokach M.D., Goodband R.D., Nelssen J.L., and Kats L.J. 1992. Influence of weaning weight and growth during the first week postweaning on subsequent pig performance. p 19-21. Kansas State University, Kansas.

Turner S.P., Farnworth M.J., White I.M.S., Brotherstone S., Mendl M., Knap P., Penny P., and Lawrence A.B. 2006. The accumulation of skin lesions and their use as a predictor of individual aggressiveness in pigs. Applied Animal Behaviour Science 96: 245-259.

Van den Brand H., Wamsteeker D., Oostindjer M., Kemp B., Bolhuis J.E., Van Enckevort L.C.M., and Van der Poel A.F.B. 2014. Effects of pellet diameter during and after lactation on feed intake of piglets pre- and postweaning. Journal of Animal Science 92: 4145-4153.

Van Nieuwamerongen S.E., Bolhuis J.E., Van der Peet-Schwering C.M.C., and Soede N.M. 2014. A review of sow and piglet behaviour and performance in group housing systems for lactating sows. Animal 8: 448-460.

Weary D.M., Jasper J., and Hötzel M.J. 2008. Understanding weaning distress. Applied Animal Behaviour Science 110: 24-41.

Weng R.C., Edwards S.A., and Hsia L.C. 2009. Effect of individual, group or ESF housing in pregnancy and individual or group housing in lactation on sow behavior. Asian-Australasian Journal of Animal Sciences 22: 1574-1580.

Widowski T.M., Torrey S., Bench C.J., and Gonyou H.W. 2008. Development of ingestive behaviour and the relationship to belly nosing in early-weaned piglets. Applied Animal Behaviour Science 110: 109-127. 
Appendix 1. Ethogram used for behaviour observations pre- and post-weaning.

\begin{tabular}{|c|c|}
\hline Behaviour & Description \\
\hline \multicolumn{2}{|l|}{ Play behaviour } \\
\hline Individual play & $\begin{array}{l}\text { Scampering (forward hops in rapid succession), turning (rapid turn on the } \\
\text { spot), head tossing, flopping (rapid drop from an upright position to } \\
\text { lying), rolling on back, sliding, running across pen individually. }\end{array}$ \\
\hline $\begin{array}{l}\text { Social play ( } 2 \text { pigs or } \\
\text { more) }\end{array}$ & $\begin{array}{l}\text { Nudging (play invitation: gentle pushing of opponent), gambolling } \\
\text { (running across pen together), play fighting, scampering together. }\end{array}$ \\
\hline Substrate play & $\begin{array}{l}\text { Shaking of head while holding material (e.g. straw, rope, faeces) that } \\
\text { protrudes from mouth (not scored when only chewing on material). }\end{array}$ \\
\hline \multicolumn{2}{|l|}{ Manipulating behaviour } \\
\hline Manipulating ears & Nibbling, sucking or chewing an ear of another piglet. \\
\hline Manipulating tails & Nibbling, sucking or chewing the tail of another piglet. \\
\hline Manipulating sow ${ }^{1}$ & Nibbling, sucking or chewing part of the body of the sow. \\
\hline Manipulating other & $\begin{array}{l}\text { Nibbling, sucking or chewing another part of the body of another piglet, } \\
\text { e.g. a paw ('hair sucking/nibbling' not scored). }\end{array}$ \\
\hline Belly nosing $^{2}$ & $\begin{array}{l}\text { Rubbing belly of another pig with up and down snout movements ( } \geq 3 \text { up } \\
\text { AND down movements). Belly sucking/manipulation is scored } \\
\text { separately. }\end{array}$ \\
\hline \multicolumn{2}{|l|}{ Aggressive behaviour } \\
\hline Head knocking & $\begin{array}{l}\text { Horizontal or vertical knocking with the head or forward thrusting with } \\
\text { the snout toward another piglet (single event or short series of events). }\end{array}$ \\
\hline Biting & Biting another piglet (single event or short series of events). \\
\hline Fighting & $\begin{array}{l}\text { Intense mutual ramming or pushing (parallel or antiparallel), with or } \\
\text { without biting, in rapid succession. }\end{array}$ \\
\hline Aggression at feeder ${ }^{2}$ & $\begin{array}{l}\text { Feed-related aggression: Push, head knock or bite given at feeder (not } \\
\text { scored when e.g. a pig gives a head knock at the feeder resulting from } \\
\text { tail/ear biting/belly nosing). }\end{array}$ \\
\hline \multicolumn{2}{|l|}{$\underline{\text { Feed-directed behaviour }}^{1}$} \\
\hline Sniffing/nosing sow feed & Sniffing/nosing sow feed. \\
\hline Eating sow feed & Chewing on sow feed (jaw moves up and down). \\
\hline $\begin{array}{l}\text { Sniffing/nosing piglet } \\
\text { feed }\end{array}$ & $\begin{array}{l}\text { Sniffing/nosing piglet feed (in MS system; feed on floor scored as sow } \\
\text { feed and feed in piglet feeder as piglet feed). }\end{array}$ \\
\hline Eating piglet feed & $\begin{array}{l}\text { Chewing on piglet feed (jaw moves up and down (in MS system; feed on } \\
\text { floor scored as sow feed and feed in piglet feeder as piglet feed)). }\end{array}$ \\
\hline Drinking & Drinking from water nipple. \\
\hline
\end{tabular}

\footnotetext{
${ }^{1}$ Pre-weaning only.

${ }^{2}$ Post-weaning only.
} 
Appendix 2. Pre- and post-weaning damage scoring protocol.

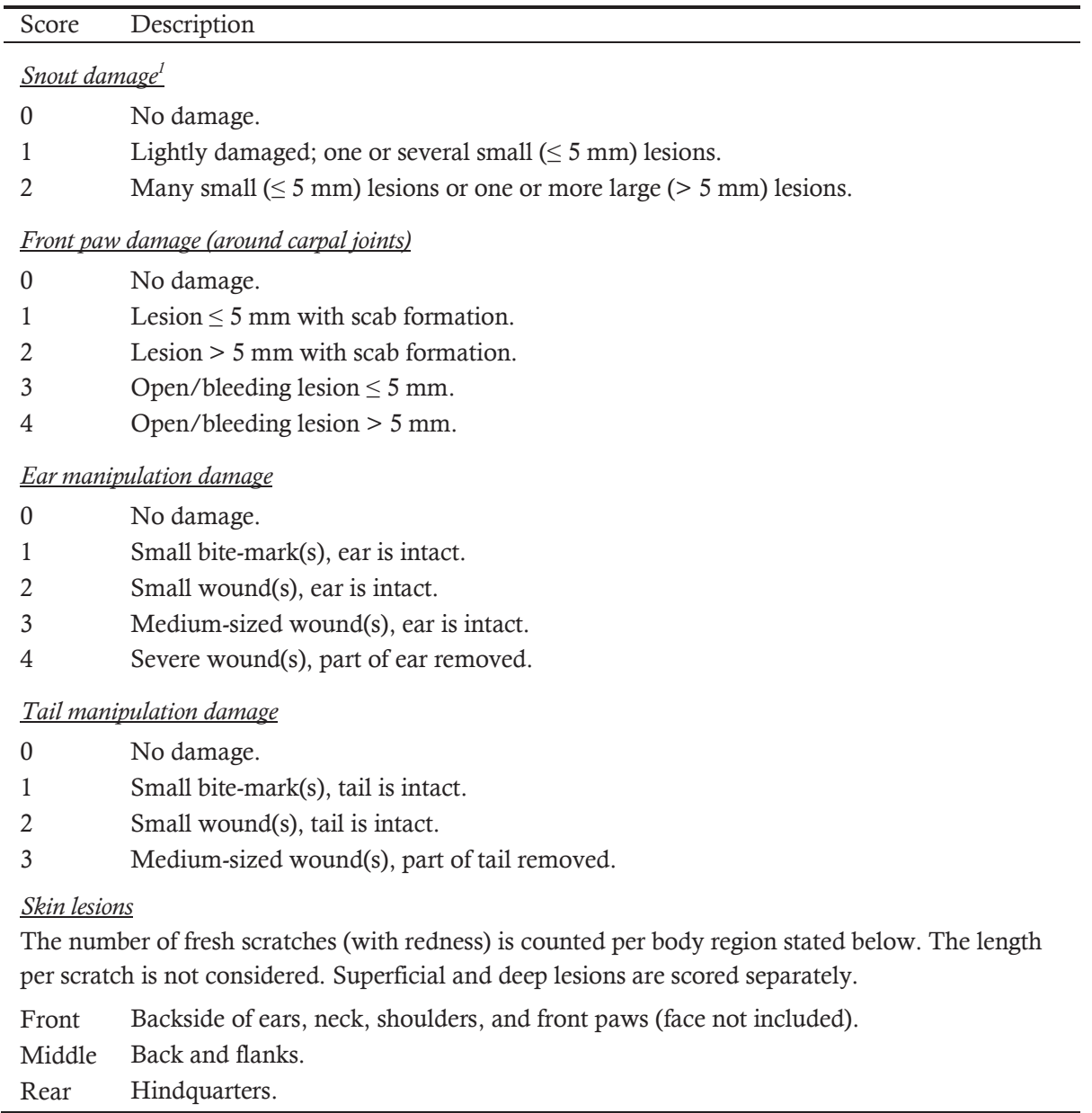

${ }^{1}$ Pre-weaning only. 


\section{CHAPTER 4}

\section{Effects of early life conditions on measures of piglet performance around weaning}

S.E. van Nieuwamerongen ${ }^{1 *}$, J.E. Bolhuis ${ }^{1}$, C.M.C. van der PeetSchwering $^{2}$, B. Kemp ${ }^{1}$, N.M. Soede ${ }^{1}$

${ }^{1}$ Adaptation Physiology Group, Department of Animal Sciences, Wageningen University \& Research, PO Box 338, 6700 AH Wageningen, The Netherlands; ${ }^{2}$ Wageningen Livestock Research, PO Box 338, 6700 AH Wageningen, The Netherlands.

*Corresponding author: S.E. van Nieuwamerongen at Sofie.vannieuwamerongen@wur.nl.

Submitted to Animal (2017) 


\subsection{Abstract}

The low feed intake and stress associated with abrupt weaning in conventional pig farming often result in poor post-weaning performance, which is related to impaired intestinal function. We investigated effects of pre-weaning housing conditions on performance around weaning of relatively light and heavy piglets. Pre-weaning, piglets were housed either with 5 sows and their litters in a multi-suckling (MS) system or in pens with individually housed sows in farrowing crates (FC). After weaning at 4 weeks of age (day 0), 16 groups of 4 piglets ( 2 light and 2 heavy litter-mates) were housed under equal conditions in enriched pens. Mannitol (day -5) and galactose (day -5 and day 5) were orally administered as markers for gastrointestinal carbohydrate absorption, and after 20 minutes a blood sample was taken (sugar absorption test). Additionally, body weight, feed intake, and faecal consistency as an indicator for diarrhoea, were assessed frequently during 2 weeks post-weaning. Pre-weaning housing, weight class, and their interaction did not affect post-weaning faecal consistency scores. Weight gain over 2 weeks did not differ between preweaning housing treatments, but MS piglets gained more $(0.67 \pm 0.12 \mathrm{~kg})$ than FC piglets $(0.39 \pm 0.16 \mathrm{~kg})$ between days 2 and 5 post-weaning $(P=0.02)$, particularly in the 'heavy' weight class (interaction, $P=0.04$ ), whereas feed intake was similar for both treatments. This indicates a better utilisation of the ingested feed of the MS piglets compared with the FC piglets in the early postweaning period. Pre-weaning mannitol concentrations were unaffected by preweaning housing, weight class, and their interaction. On day 5 post-weaning, however, MS piglets had a lower plasma concentration of mannitol (320 \pm 116 vs. $592 \pm 120 \mathrm{nmol} / \mathrm{mL}, P=0.04)$ and galactose $(91 \pm 18$ vs. $157 \pm 19$ $\mathrm{nmol} / \mathrm{mL}, P=0.04)$ than FC piglets, regardless of weight class. In conclusion, MS and FC piglets differed in aspects of post-weaning gastrointestinal carbohydrate absorption, but pre-weaning housing did not affect overall feed intake, weight gain and measures of faecal consistency over the first 2 weeks after weaning.

Key words: intestinal function, group housing, multi-suckling system, pigs, weaning 


\subsection{Introduction}

At weaning in conventional pig farming, piglets are removed from the sow at 3 to 4 weeks of age, and consequently have to switch from ingesting mainly sow milk, with fat and lactose as major energy sources (Hurley, 2015), to eating solid feed which contains more complex carbohydrates. This abrupt dietary switch, in combination with other stressors such as changes in the piglets' social and physical environment, generally results in a low post-weaning feed intake (Vente-Spreeuwenberg and Beynen, 2003), with fasting periods up to 4 days in some piglets (Bruininx et al., 2001). The lack of nutrients in the intestinal lumen leads to changes in intestinal morphology, such as a reduced villus height, an increased crypt depth, and an increased paracellular permeability (Hampson, 1986; van Beers-Schreurs et al., 1998; Spreeuwenberg et al., 2001). The latter can contribute to an impaired intestinal barrier function after weaning, facilitating bacteria and toxins to cross the epithelial barrier (Vente-Spreeuwenberg and Beynen, 2003). Taken together, the post-weaning intestinal capacity to digest and absorb nutrients is suboptimal and the protective barrier function of the gut is compromised in conventionally weaned piglets (Wijtten et al., 2011). This contributes to their poor post-weaning performance, which is characterised by e.g. a growth check and the occurrence of diarrhoea (Pluske et al., 1997).

To ease the transition from the milk-based diet in the suckling phase to the post-weaning diet, it is important to stimulate pre-weaning feed intake (Bruininx et al., 2002; Berkeveld et al., 2007). Piglets offered creep feed during lactation (Meyer, 2008) and/or characterised as pre-weaning 'eaters' (Sulabo et al., 2010) showed a better post-weaning performance. Moreover, creep feeding during lactation increased net absorption in the small intestine after weaning (Kuller et al., 2007), suggesting that early experience with solid feed increases piglets' capacity to cope with the dietary switch after weaning. In a previous experiment (van Nieuwamerongen et al., 2015), we found that piglets raised in a multi-litter system showed an improved early post-weaning performance compared with conventionally kept piglets, which was reflected in higher feed intake, higher weight gain, and indicators of a lower diarrhoea occurrence. The pre-weaning environment in the multi-litter system was likely an important contributing factor, as there were ample opportunities for social facilitation and social learning of eating (Nicol and Pope, 1994; Morgan et al., 2001; 
Oostindjer et al., 2011) in the communal feeding area, and the presence of enrichment materials may have stimulated the development of chewing and eating behaviour (Oostindjer et al. 2014). Post-weaning housing, however, also differed between both treatment groups in our previous work, hence the relative contribution of pre-weaning and post-weaning conditions regarding post-weaning performance could not be distinguished. Moreover, it was not known to what extent a difference in intestinal function may have contributed to the observed differences. Therefore, the aim of the current study was to clarify the impact of these pre-weaning housing conditions (i.e. a multisuckling system vs. individual pens with crated sows) on peri-weaning performance and measures of intestinal function under equal post-weaning housing conditions. This was studied in relatively light and relatively heavy piglets, as these may differ in their response to pre-weaning conditions (Meyer, 2008).

\subsection{Materials and methods}

\subsubsection{Animals and experimental design}

The experiment was approved by the Animal Care and Use Committee of Wageningen University \& Research (AVD10400201551, registration code 2015024.c). In total, 64 piglets (Tempo x Topigs 20) from 16 litters were studied in 2 successive batches. Before weaning, piglets were housed at the animal facilities of Swine Innovation Centre Sterksel, the Netherlands, either in a multi-suckling (MS) system consisting of 5 sows and their litters or with a sow in a conventional farrowing crate (FC). All sows were multiparous and allocation of the sows to the pre-weaning housing treatments was balanced for parity. Per batch and per housing system, 4 healthy litter-mates from 4 litters were selected to participate in this study. Per litter, these selected piglets consisted of 1 light and 1 heavy piglet from both sexes. Selection took place based on body weight 6 days before weaning (day -6). In principle, the lightest and heaviest piglet from both sexes was selected, with the exception of outliers considering the lightest piglets. In addition, when comparing the selected animals with the intact group, a similar relative weight difference was strived for per pre-weaning housing treatment. Before selection, mean litter weights at day -6 were $6.39 \pm 0.30 \mathrm{~kg}$ in the MS system and $6.16 \pm 0.34 \mathrm{~kg}$ in the FC system. Mean body weight of the selected piglets was $7.53 \pm 0.23 \mathrm{~kg}$ for the 
'heavy' MS piglets, $5.57 \pm 0.23 \mathrm{~kg}$ for the 'light' MS piglets, $7.34 \pm 0.23 \mathrm{~kg}$ for the 'heavy' FC piglets, and $5.44 \pm 0.20 \mathrm{~kg}$ for the 'light' FC piglets. After weaning at $27.1 \pm 0.4$ days of age, the piglets were transported to the research facility 'Carus' of Wageningen University, the Netherlands. Post-weaning housing conditions were the same for all piglets.

\subsubsection{General pre-weaning management}

Piglets were ear tagged and weighed within 24 hours post-partum (p.p.). Litter sizes were standardised between 24 and 48 hours p.p. according to the number of functional teats available per sow. Piglets were tail docked and received an iron injection within 4 days after birth. Male piglets were not castrated. Prestarter creep feed (11.6 MJ of NE, 17.5\% crude protein, and 1.17\% ileal digestible lysine) was provided to the piglets in round feeders (diameter $28 \mathrm{~cm}$ ) twice daily from 12 days after the expected date of birth. On days 21 and 22 pre-starter was gradually mixed with weaner feed (9.9 MJ of NE, 16.0\% crude protein, and $0.99 \%$ ileal digestible lysine), after which only weaner feed was provided. Water was available ad libitum and animal health was checked twice daily. Litter size at weaning was $10.5 \pm 0.5$ in the MS system and $12.6 \pm 0.5$ in the FC system $(P=0.14)$. Litter sizes at weaning were (numerically) lower due to higher mortality in the MS system, despite peri-natal litter equalisation due to cross-fostering.

\subsubsection{Pre-weaning housing}

\section{Multi-suckling system}

MS housing consisted of 5 farrowing pens connected to a communal area (Figure 4.1). Sows could access all areas from the moment of entry in the system. The farrowing pens were $3.2 \times 2.2 \mathrm{~m}$ each and contained a feed trough for the sow, a water nipple for the piglets, and a covered piglet nest of $0.7 \times 1.6$ $\mathrm{m}$ with heated solid flooring. The floor in the rest of the pen consisted of solid concrete and concrete slats. Per farrowing pen, 5 hessian sacks were provided as nesting material. From day 2 p.p., 2 handfuls of long-stemmed straw were provided daily per pen. The adjacent communal area was divided in an area for feeding (solid concrete and metal slats), defecating/urinating (metal slats), and lying (solid concrete and metal slats). Five hessian sacks and 5 ropes were attached to the partitions surrounding the resting area and were replaced if 


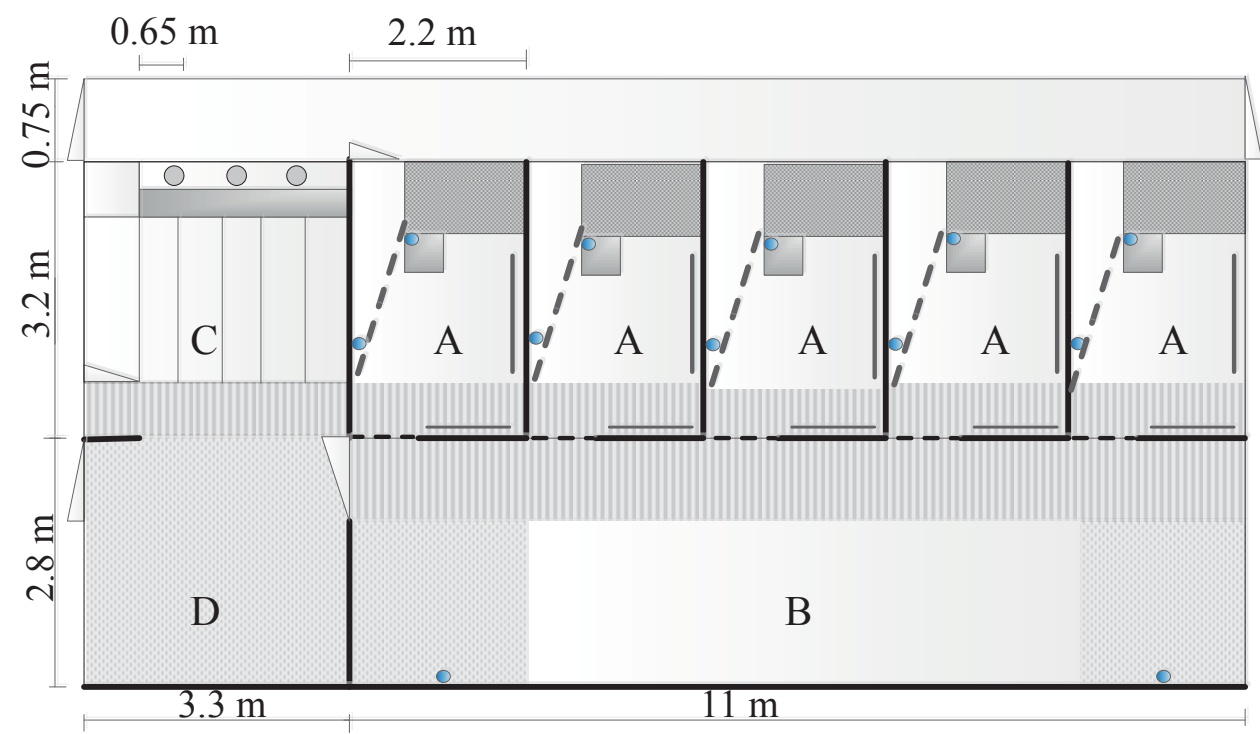

Figure 4.1. Layout of the multi-suckling system. The system has 5 farrowing pens (A with piglet nest, sow feeder, drinkers, and anti-crushing devices) connected to a communal area with a lying area (B - with 2 drinkers), feeding area (C - with 5 sow feeding places surrounded by a piglet area with piglet feeders), and a dunging area (D).

needed. The communal feeding area contained 5 feeding places for the sows separated by horizontal metal bars, and a surrounding area with piglet feeders accessible to only the piglets. Each individual sow was locked in her own farrowing pen daily between $16: 30$ and $07: 30 \mathrm{~h}$ until farrowing of that particular sow had ended. From that moment onwards, each sow could freely access all farrowing pens and communal areas again. Piglets were given access to the whole system at a mean age of $7.9 \pm 0.3$ days. This was achieved by removing a $31 \mathrm{~cm}$ high piglet barrier at the entrance from each farrowing pen. In addition, a 'piglet door' $(0.4 \times 0.3 \mathrm{~m})$ was removed from the front wall of each farrowing pen, to provide piglets more space to move in and out of the farrowing pens. The sows were floor fed twice daily in the communal area, and piglets could eat together with the sows from the sow feed and from creep feed provided in the piglet feeders. Sows were fed in their own farrowing pen only in the afternoons before farrowing and in the first days after farrowing in case the sows did not leave their pen to eat in the communal area. Otherwise, they were fed in the communal area. 


\section{Conventional system}

Each FC litter was kept in a pen of $2.4 \times 1.8 \mathrm{~m}$, which contained a farrowing crate of $2.0 \times 0.7 \mathrm{~m}$ for the sow. The pens were situated in farrowing units containing 12 pens each. The floor consisted of metal slats within the crate, a solid floor of $1.2 \times 0.3 \mathrm{~m}$ with a heat lamp for the piglets and plastic slats in the remaining area. The sow had a feed trough and a drinking nipple available. The piglets had access to a separate drinking nipple and creep feed was provided in a round feeder located in the corner at the posterior side of the sow. One day prior to expected farrowing, sows received a hessian sack as nesting material. During the whole pre-weaning period, a plastic roll was available for both sow and piglets, and a chain with a wooden block was available as enrichment for the sow.

\subsubsection{Post-weaning housing}

After weaning, 32 piglets per batch were transported (for about 1.5 hours) to their new housing facilities. During transport, the $16 \mathrm{MS}$ piglets were penned in 1 group, whereas the $16 \mathrm{FC}$ piglets were penned in groups of 4 litter-mates. After arrival, MS and FC litters were equally divided over 2 adjacent rooms, where the 4 pens in 1 room were filled alternatingly with piglets from MS and FC litters. Each pen $(2.7 \times 3.6 \mathrm{~m})$ housed 4 litter-mates and was bedded with $550 \mathrm{~L}$ of sawdust, $10 \mathrm{~kg}$ of straw, and $90 \mathrm{~L}$ of peat on a solid floor. A physically enriched post-weaning environment was provided, as experiencing a reduction in the level of environmental enrichment may be detrimental (see e.g. Bolhuis et al., 2006; Munsterhjelm et al., 2009), which would occur if the MS piglets would be housed under more conventional conditions. On a daily basis, soiled bedding was removed from the pens and $70 \mathrm{~L}$ of fresh sawdust and $1 \mathrm{~kg}$ of straw was added. Fresh peat was added on a weekly basis (45 L). A hessian sack, a rope and a chain with bolts were available as further enrichment. The same weaner feed that was provided in the late pre-weaning phase was offered ad libitum in a feeder with 4 eating places. Water was continuously available from a drinking nipple. Animal health was checked twice daily. 


\subsubsection{Measurements}

Piglets were weighed on days -6, -1, 2, 5, and 13 post-weaning. Post-weaning feed intake at pen level was determined for days 0 to 2,2 to 5 , and 5 to 13 . A faecal consistency score was given to all piglets on days $-1,2,5,7$, and 9 postweaning by means of visual inspection of the anal area of each piglet. A score of 0 was given if no faeces was present, score 1 if pasty faeces was present, and score 2 if watery faeces was present. Furthermore, piglets were subjected to a sugar absorption test using a protocol based on Turpin et al. (2016) on day 5 before weaning and day 5 after weaning. Piglets were fasted for 3 hours, during which they had access to drinking water but not to solid feed or milk. Before weaning, piglets were fasted by relocating the 4 litter-mates to an empty pen in a different unit. After weaning, piglets were fasted by removing the feeders from the pens. After the fasting period, each piglet was fixated in a vertical position with the nose pointing upward. Then, a nasogastric tube lubricated with paraffin oil was inserted in the stomach via the nose, after which physiological saline was administered. Thereafter, a sugar solution was administered. On day 5 before weaning, all piglets received $5 \mathrm{ml} / \mathrm{kg}$ body weight of $10 \%$ mannitol. On day 5 after weaning, half of the piglets (balanced for pre-weaning housing system, weight class, and sex) received $5 \mathrm{ml} / \mathrm{kg}$ body weight of $10 \%$ galactose and the other half received $5 \mathrm{ml} / \mathrm{kg}$ body weight $10 \%$ mannitol. Galactose was administered only after weaning because of the presence of galactose in milk. Thereafter, the piglet was placed back with its litter-mates. Twenty minutes after administration of the sugar solution, the piglet was fixated in a supine position by 2 persons and a $4 \mathrm{ml}$ blood sample was collected in a heparin tube by puncture of the jugular vein. The sample was stored on ice (max. 1 hour) until further processing. When the 4 piglets of 1 litter had been blood sampled, they were placed back in their home pen (before weaning) or the feeder was placed back in their pen (after weaning). Samples were centrifuged for 10 minutes at $1,300 \mathrm{~g}$ at $4^{\circ} \mathrm{C}$ and plasma was stored at $-20^{\circ} \mathrm{C}$ until analysis. Mannitol concentrations were determined using a commercial colorimetric assay kit (Abcam, ab155890). D-mannitol was converted to D-fructose by mannitol dehydrogenase in the presence of NAD to form $\mathrm{NADH}$, which reduces a colourless probe to a chromogen with strong absorbance at $450 \mathrm{~nm}$. Galactose concentrations were determined using a commercial assay kit (Abcam, ab83382) in which galactose is specifically oxidised generating a product that produces colour (OD570 nm). For both 
sugars, concentrations were calculated using their corresponding standard curve, in accordance with the manufacturer's instructions.

\subsubsection{Statistical analysis}

Data were analysed with SAS 9.2 (SAS Inst. Inc., Cary, NC). Residuals were checked for normality and variables were transformed with a square root or logarithm if needed. Body weight gain, sugar concentrations, and the number of days piglets had pasty or watery faeces were analysed with mixed models including pre-weaning housing system, weight class, housing $\mathrm{x}$ weight class interaction, and batch. Pen within housing treatment was included as a random effect, as measures on pigs from the same litter and pen may not be independent.

Feed intake was determined at pen level and was analysed with mixed models including pre-weaning housing system and batch. One FC pen in the second batch was excluded from analysis of feed intake because piglets in this pen had a poor start after weaning with severe diarrhoea and therefore received liquid feed supplementation. Body weight was analysed with a mixed model including pre-weaning housing system, day post-weaning, weight class, their 2way and 3-way interactions, and batch. Pen (within housing treatment and batch) was included as a random effect. Repeated observations on the same animals were taken into account by including a repeated effect of piglet (within pen, housing treatment, and weight class). The proportion of piglets with pasty or watery faeces was analysed with generalised linear mixed models using a binomial distribution and logit link function, including pre-weaning housing system, weight class, housing $\mathrm{x}$ weight class interaction, and batch. Pen within housing treatment was included as a random effect. Average faecal consistency scores were analysed with mixed models including pre-weaning housing system, day post-weaning, weight class, their 2-way and 3-way interactions, and batch. Pen (within housing treatment and batch) was included as a random effect. Repeated observations on the same animals were taken into account by including a repeated effect of piglet (within pen, housing treatment, and weight class). Sex did not affect any of the variables tested and was therefore removed from the final models. Results are presented as means \pm SEM. $P$-values below 0.05 were considered statistically significant. 


\subsection{Results}

\subsubsection{Body weight, feed intake, and faecal consistency scores}

Pre-weaning housing $(P=0.13)$, housing $\mathrm{x}$ weight class interaction $(P=0.56)$, housing $\mathrm{x}$ day interaction $(P=0.84)$, weight class $\mathrm{x}$ day interaction $(P=0.93)$, and housing $\mathrm{x}$ weight class $\mathrm{x}$ day interaction $(P=0.99)$ did not affect postweaning body weight (Figure 4.2). 'Heavy' piglets, however, had a higher body weight than 'light' piglets on all days measured and body weight increased over time (Figure 4.2). Between days -1 to 2 , 'heavy' MS piglets tended to have a lower weight gain than 'light' MS piglets $(P=0.09$ for the interaction between pre-weaning housing and weight class, see Figure 4.3$)$. Between days 2 to 5 , weight gain was higher for MS piglets compared with FC piglets $(0.67 \pm 0.12$ vs. $0.39 \pm 0.16, P=0.02$ ). This difference, however, seemed to hold mainly for piglets in the 'heavy' weight class; 'heavy' MS piglets gained more weight than both 'heavy' and 'light' FC piglets, and 'heavy' FC piglets gained less than both 'heavy' and 'light' MS piglets $(P=0.04$ for the interaction between preweaning housing and weight class, see Figure 4.3 ). When weight class was excluded from the statistical model, the housing effect remained significant $(P$ $=0.02$ ). Pre-weaning housing, weight class, and their interaction did not affect weight gain between days 5 to 13 and days -1 to 13 (Figure 4.3). Mean overall weight gain between days -1 to 13 was $3.27 \pm 0.16 \mathrm{~kg}$ per MS piglet and $3.00 \pm$ $0.38 \mathrm{~kg}$ per FC piglet. Feed intake between days 0 to 2, 2 to 5, 5 to 13, and 0 to 13 did not differ between pre-weaning housing treatments (Table 4.1) and neither did feed conversion between days 0 to 13 (MS: $1.43 \pm 0.10$, FC: $1.43 \pm$ $0.14, P=0.86$ ). Lastly, pre-weaning housing, weight class, and their interaction did not affect measures related to faecal consistency (Table 4.2 and Figure 4.4).

Table 4.1. Feed intake per piglet $(\mathrm{kg})$ relative to the day of weaning (= day 0 ) of piglets raised in a multi-suckling system (MS) and piglets raised with a sow in a farrowing crate (FC). $P$-values are given for effects of pre-weaning housing.

\begin{tabular}{lccc}
\hline Day & MS & FC & Housing \\
\hline Days 0 to 2 & $0.14 \pm 0.03$ & $0.18 \pm 0.05$ & 0.54 \\
Days 2 to 5 & $0.76 \pm 0.05$ & $0.78 \pm 0.12$ & 0.74 \\
Days 5 to 13 & $3.72 \pm 0.25$ & $3.61 \pm 0.32$ & 0.89 \\
Days 0 to 13 & $4.61 \pm 0.24$ & $4.56 \pm 0.20$ & 0.98 \\
\hline
\end{tabular}




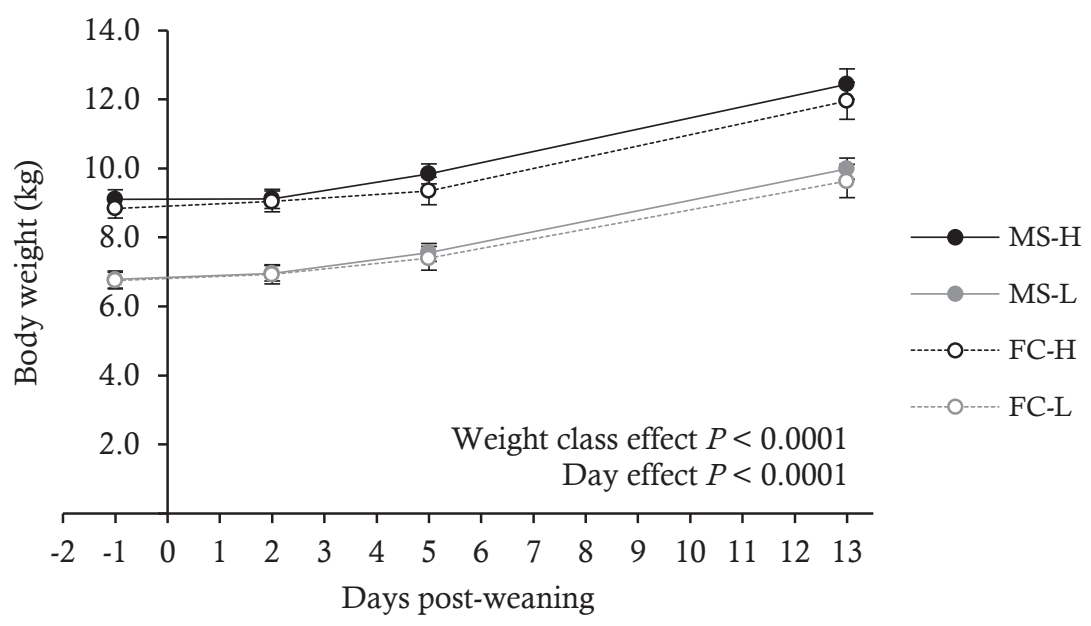

Figure 4.2. Body weight $(\mathrm{kg})$ relative to the day of weaning (= day 0$)$ of piglets raised in a multi-suckling system (MS) and piglets raised with a sow in a farrowing crate (FC) per weight class determined on day $-6(\mathrm{H}=$ heavy and $\mathrm{L}=$ light $)$.

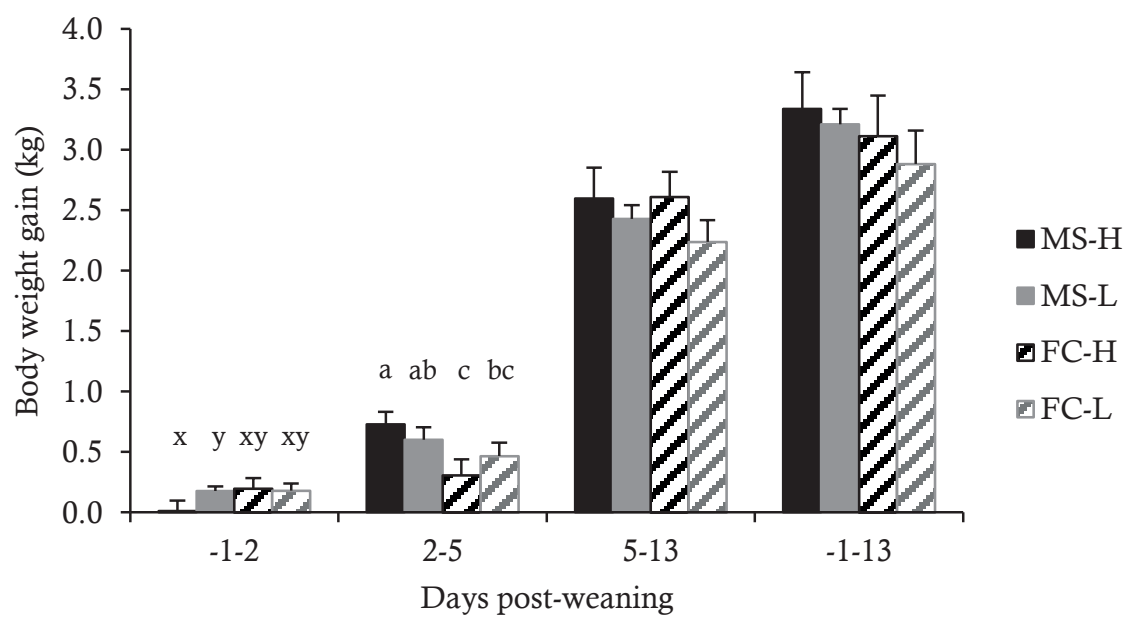

Figure 4.3. Body weight gain relative to the day of weaning (= day 0 ) of piglets raised in a multi-suckling system (MS) and piglets raised with a sow in a farrowing crate (FC) per weight class determined on day $-6(\mathrm{H}=$ heavy and $\mathrm{L}=$ light $)$. Values without a common letter differ at a significance level of $P<0.05$ (abc) or $P<0.10$ (xy). 


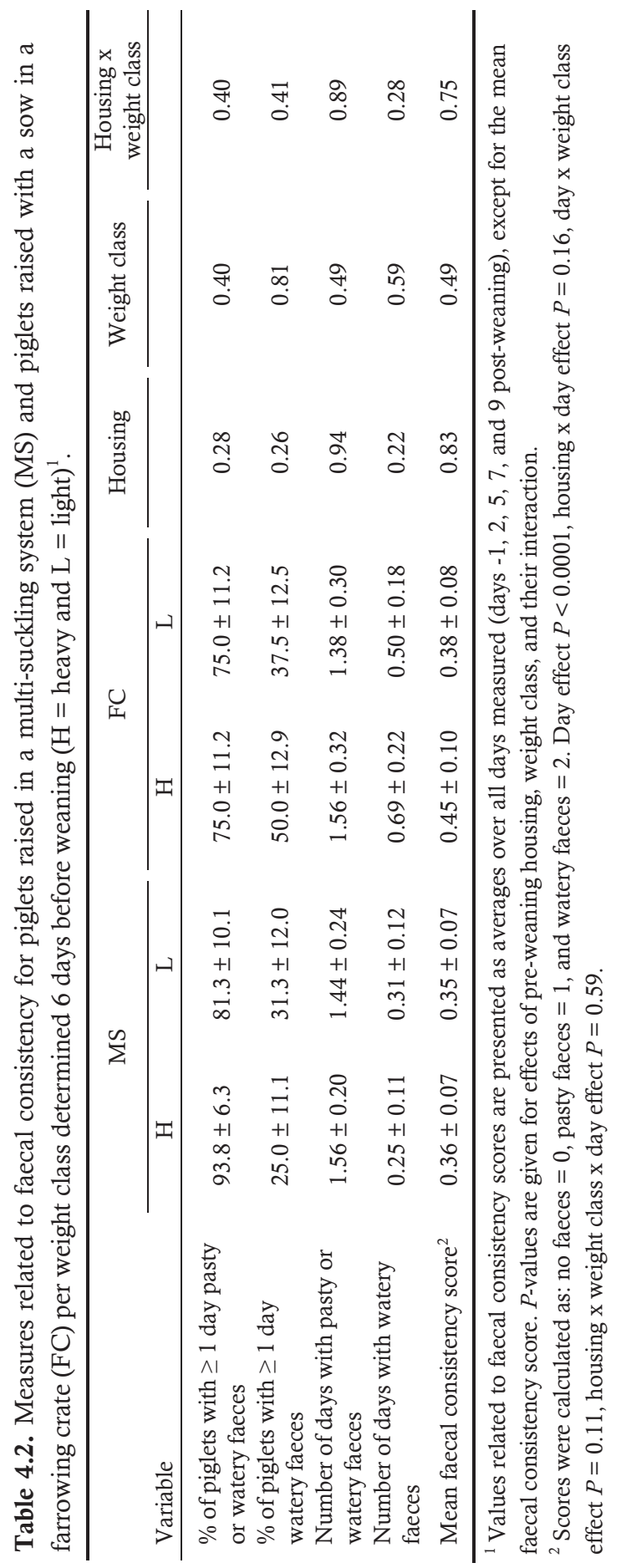




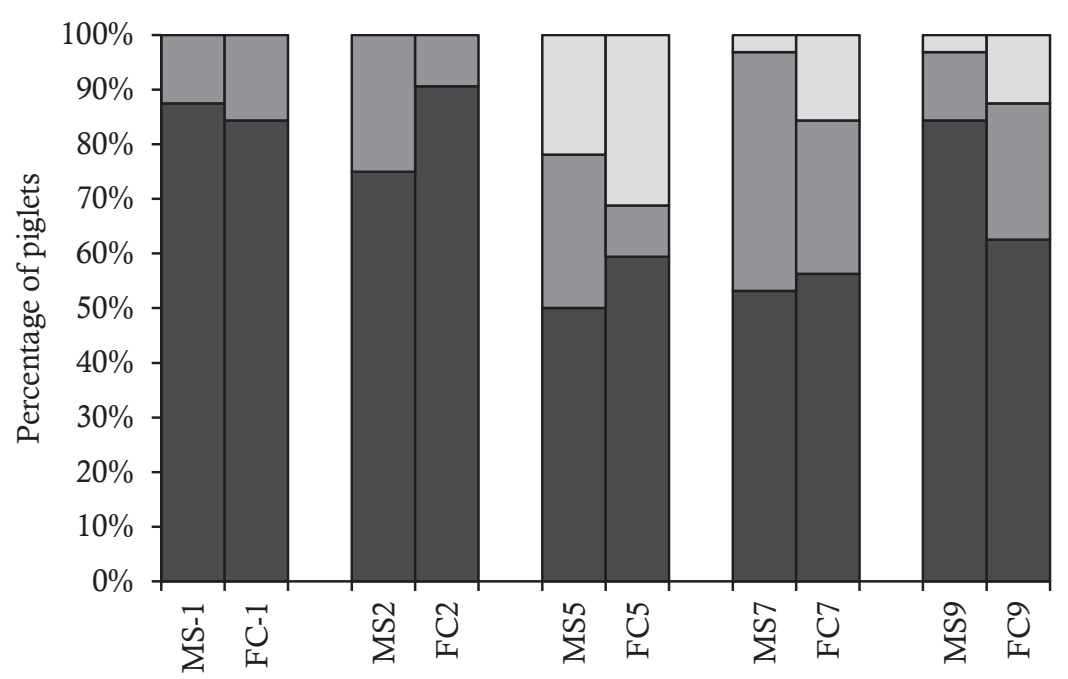

Figure 4.4. Faecal consistency scores on days -1, 2, 5, 7, and 9 post-weaning for piglets raised in a multi-suckling system (MS) and piglets raised with a sow in a farrowing crate (FC). A faecal consistency score was given by means of visual inspection of the anal area of each piglet. A score of 0 was given if no faeces was present $(\square)$, score 1 if pasty faeces was present $(\square)$, and score 2 if watery faeces was present $(\square)$.

\subsubsection{Sugar absorption test}

Pre-weaning mannitol concentrations 20 minutes after oral administration on day -5 were not affected by pre-weaning housing, weight class, or their interaction (Figure 4.5). On day 5 post-weaning, MS piglets had a lower plasma concentration of mannitol $(320 \pm 116$ vs. $592 \pm 120 \mathrm{nmol} / \mathrm{mL}, P=$ $0.04)$ and galactose $(91 \pm 18$, vs. $157 \pm 19 \mathrm{nmol} / \mathrm{mL}, P=0.04)$ than FC piglets. When the FC pen that had a poor start after weaning was excluded from analysis, the housing effect on galactose remained significant, but that on mannitol became a tendency $(320 \pm 116$ vs. $506 \pm 97 \mathrm{nmol} / \mathrm{mL}, P=0.07)$. Post-weaning sugar concentrations were unaffected by weight class and housing $\mathrm{x}$ weight class interaction (Figure 4.5).

\subsection{Discussion}

Our study aimed to gain more insight in the impact of pre-weaning housing conditions on peri-weaning performance, including measures of intestinal function. Pre-weaning housing consisted of either a multi-suckling system or 


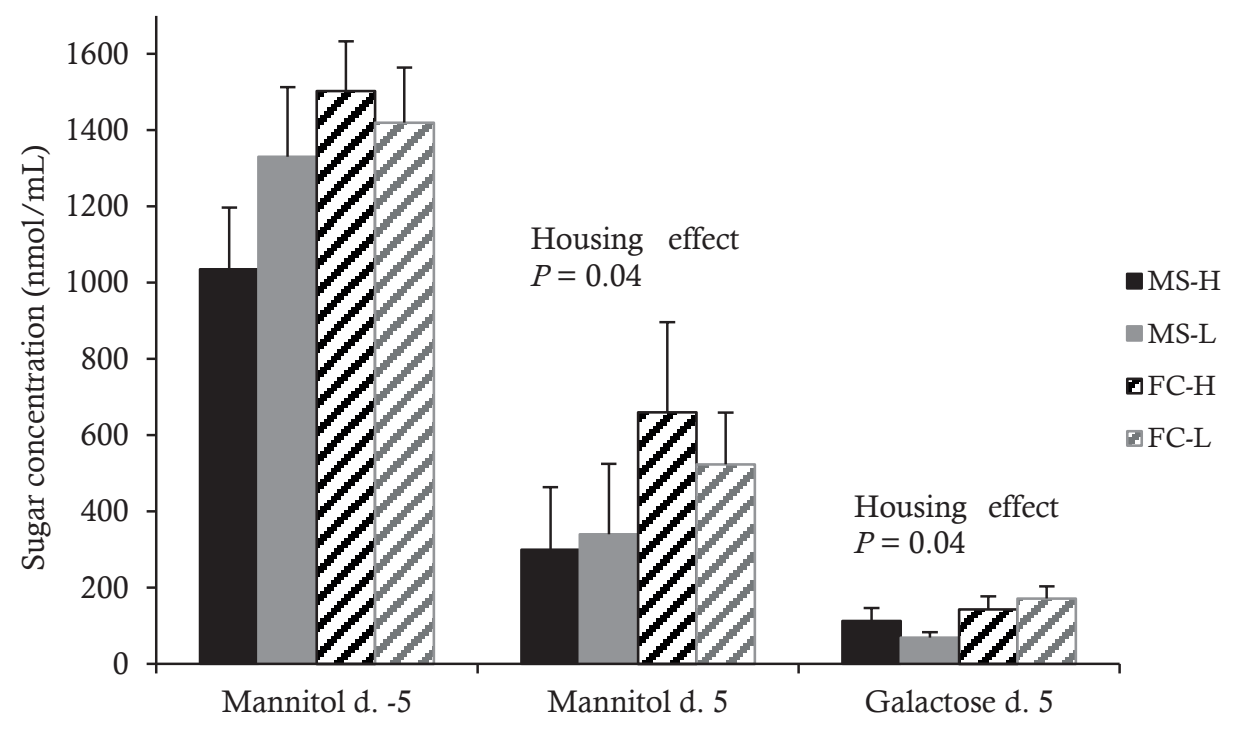

Figure 4.5. Plasma sugar concentrations 20 minutes after oral administration, relative to the day of weaning (= day 0 ), of piglets raised in a multi-suckling system (MS) and piglets raised with a sow in a farrowing crate $(\mathrm{FC})$ per weight class determined on day $-6(\mathrm{H}=$ heavy and $\mathrm{L}=$ light).

individual pens with crated sows, and post-weaning conditions consisted of an enriched environment that was equal for all piglets. Overall, post-weaning faecal consistency scores, feed intake, body weight, and weight gain were similar in piglets from both pre-weaning housing treatments. Between days 2 to 5 post-weaning, however, the MS piglets had a $72 \%$ higher weight gain than the FC piglets. This is consistent with a previous study (van Nieuwamerongen et al., 2015). The difference in weight gain between MS and FC piglets, whilst having a similar feed intake, suggests a different efficiency of digestion and absorption of nutrients. Between days 2 to 5 post-weaning, feed conversion was lower in MS piglets than in FC piglets $(1.34 \pm 0.17$ vs. $1.78 \pm 0.24, P=0.02)$. Possibly, the use of the communal feeding area in the MS system played a role, where piglets were stimulated to start eating at an early age. Abrupt weaning at a relatively young age of 4 weeks, compared with the gradual weaning process seen in nature, forces the gastrointestinal tract into an accelerated maturation (Miller and Slade, 2003). In a previous study, we observed that MS piglets showed an almost threefold higher frequency of feed directed behaviour at 2 weeks of age than FC piglets (van Nieuwamerongen et al., 2015). Possibly, the MS piglets in the current experiment also experienced earlier contact with solid 
feed before weaning due to social learning and facilitation, more gradually preparing the intestinal tract for digestion and absorption of solid feed.

Other post-weaning differences that were found in an earlier study (van Nieuwamerongen et al., 2015) were not confirmed in the current study. In the previous study, piglets raised in an MS system showed a higher feed intake between days 0 to 2 post-weaning $(0.44 \pm 0.05$ vs. $0.30 \pm 0.04 \mathrm{~kg}$ per piglet, $P$ $<0.01)$, and a higher post-weaning weight gain $(3.81 \pm 0.37$ vs. $2.58 \pm 0.23 \mathrm{~kg}$ per piglet, $P<0.05$, days -1 to 13 ), and more solid faecal consistency scores over the first 2 weeks after weaning. This discrepancy likely results from differences between both studies in post-weaning housing conditions, which made the transition from pre-weaning to post-weaning housing in the current study less favourable for the MS piglets than for the FC piglets. For the MS piglets, the large changes in group size, group composition, and total available space at weaning may have contributed to their relatively poorer performance compared with the previous study (where MS piglets were housed in groups of 40 with some enrichment post-weaning). For the FC piglets, on the other hand, the post-weaning pen was substantially more enriched and larger than their farrowing pen, making the transition more favourable than in the previous study (where FC piglets were housed in groups of 10 litter-mates in a barren pen). Pigs' performance in a particular housing environment depends on their previous experiences, with negative effects if the current environment is less enriched than the previous one (Bolhuis et al., 2006; Munsterhjelm et al., 2009). The enriched post-weaning conditions in the current study may therefore have reduced the contrast between MS and FC animals.

The relationship between coping with weaning and piglet weight is equivocal. On the one hand, it is suggested that heavy piglets are more mature and tend to have higher pre-weaning creep feed intake and higher pre- and post-weaning weight gain. On the other hand, light piglets may show more compensatory eating before weaning, making the transition to the post-weaning diet easier (Pajor et al., 1991). In our study, there were no large differences between relatively heavy and light piglets. 'Light' piglets had a lower body weight than 'heavy' piglets on all days measured, but weight gain was similar in both weight classes. There was, however, (a tendency for) an interaction between pre-weaning housing and weight class between days -1 to 2 and days 2 to 5 . 'Heavy' MS piglets showed a very low weight gain between days -1 and 2, but 
numerically had the highest weight gain between days 2 to 5 . The 'heavy' FC piglets seemed to lag behind in weight gain between days 2 to 5 .

Considering the results of the sugar absorption test; it has been reported that mannitol transport mainly occurs via solvent drag, i.e. by water movement driven by osmotic or electrochemical gradients, carrying along small molecules through the paracellular pathway (Krugliak et al., 1994; Menard et al., 2010). Under post-weaning circumstances, where intestinal function is likely compromised, it would be expected that the gradients are lower than in a healthy intestine in which nutrients are digested and absorbed efficiently. It would then be expected that in a damaged post-weaning intestine, mannitol levels are lower than before weaning. Moreover, it has been reported that increased mannitol absorption can indicate a larger small intestinal surface area (Sigalet et al., 2000). If so, post-weaning mannitol levels are expected to be lower than pre-weaning levels, as a reflection of a decreased intestinal surface area resulting from weaning. This seems to be in line with the numerically lower levels of mannitol after weaning compared with before weaning in our study, and similar results were found by Turpin et al. (2016). However, this does not provide a satisfactory explanation for the lower plasma concentrations of mannitol (-46\%) on day 5 after weaning in MS piglets compared with FC piglets, as MS piglets seemed to perform better between days 2 to 5 in terms of feed utilisation. The interpretation of the different post-weaning mannitol concentrations is thus not straightforward. As mannitol has been used as a marker for intestinal permeability, it can be speculated that the higher mannitol concentrations in FC piglets indicate higher intestinal permeability by paracellular transport via the tight junctions between the epithelial cells, thus possibly reflecting a compromised intestinal barrier function (VenteSpreeuwenberg and Beynen, 2003; Wijtten et al., 2011). Stress may play a role in impairing the barrier function, mediated by receptors for corticotrophinreleasing factor in the intestine (Moeser et al., 2007). Nevertheless, we found no other indications of an impaired intestinal barrier function in the FC piglets (e.g. reflected in the faecal consistency scores). In addition, it has been reported that mannitol molecules are small enough to pass the tight junctions between cells in a healthy intestine (Menard et al., 2010; Price et al., 2015) and that transcellular, rather than only paracellular, transport may also occur (Wijtten et al., 2011). These different interpretations of mannitol concentrations may 
also depend on whether assessments were made in vitro or in vivo (Menard et al., 2010).

Besides a difference in post-weaning mannitol concentrations, we also found lower levels of galactose after weaning in MS piglets compared with FC piglets $(-42 \%)$. Galactose absorption occurs through active transport via the transcellular pathway using an $\mathrm{Na}^{+}$/galactose co-transporter (SGLT1). Active absorption may increase in response to weaning, possibly due to a fasting state, as a low availability of nutrients seems to upregulate active absorption in the proximal jejunum (Carey et al., 1994; Boudry et al., 2004; Wijtten et al., 2011) and/or due to the switch from a milk-based diet to a cereal-based diet (Boudry et al., 2002; Wijtten et al., 2011). As post-weaning feed intake did not differ between MS and FC piglets, it can be speculated that the suggested difference in onset of pre-weaning experience with solid feed (as found in our earlier work) may have affected post-weaning active nutrient absorption.

Overall, the interpretation of the results from the sugar absorption test is not straightforward as several mechanisms may play a role. Moreover, the relative contribution of each mechanism may differ before and after weaning. In addition, it should be noted that, in general, the found differences in this study may partly be explained by the numerically different litter size at weaning for MS and FC piglets. Piglet development can be affected by the level of competition at the udder, and relatively light piglets may for instance perform better in a small litter than in a large litter (English and Bilkei, 2004). Nevertheless, there was no difference between the MS and FC piglets weaning weight before and after selection of piglets.

In conclusion, MS and FC piglets differed in aspects of gastrointestinal carbohydrate absorption, but pre-weaning housing did not affect overall feed intake, weight gain, and measures of faecal consistency over the first 2 weeks after weaning. Between days 2 and 5, however, MS piglets showed a better feed utilisation, as they gained more weight whilst having a similar feed intake in this period, compared with FC piglets. Overall, this study suggests that preweaning housing conditions can affect measures of intestinal function after weaning, even with similar levels of post-weaning feed intake. 


\subsection{Acknowledgements}

The authors gratefully acknowledge the staff of Swine Innovation Centre Sterksel and Research Facility Carus for their assistance and care for the animals. Furthermore, the authors want to thank Bjorge Laurenssen, Fleur Bartels, Rudie Koopmanschap, Monique Ooms, Caroline Clouard, Nick Timmermans, Sanne Benjamins, Jasmijn Strous, and Hilde de Kok for their assistance during various parts of this experiment. 


\subsection{References}

Berkeveld M., Langendijk P., van Beers-Schreurs H.M., Koets A.P., Taverne M.A., and Verheijden J.H. 2007. Postweaning growth check in pigs is markedly reduced by intermittent suckling and extended lactation. Journal of animal science 85: 258-266.

Bolhuis J.E., Schouten W.G.P., Schrama J.W., and Wiegant V.M. 2006. Effects of rearing and housing environment on behaviour and performance of pigs with different coping characteristics. Applied Animal Behaviour Science 101: 6885.

Boudry G., Lallès J.P., Malbert C.H., Bobillier E., and Sève B. 2002. Diet-related adaptation of the small intestine at weaning in pigs is functional rather than structural. Journal of pediatric gastroenterology and nutrition 34: 180-187.

Boudry G., Péron V., Luron I., Lallès J., and Sève B. 2004. Weaning induces transient and long-lasting modifications of absorptive and secretory properties and epithelial barrier function of piglet intestine. J Nutr 134: 2256-2262.

Bruininx E.M.A.M., Binnendijk G.P., Van Der Peet-Schwering C.M.C., Schrama J.W., Den Hartog L.A., Everts H., and Beynen A.C. 2002. Effect of creep feed consumption on individual feed intake characteristics and performance of group-housed weanling pigs. Journal of Animal Science 80: 1413-1418.

Bruininx E.M.A.M., Van Der Peet-Schwering C.M.C., Schrama J.W., Vereijken P.F.G., Vesseur P.C., Everts H., Den Hartog L.A., and Beynen A.C. 2001. Individually measured feed intake characteristics and growth performance of group-housed weanling pigs: Effects of sex, initial body weight, and body weight distribution within groups. Journal of Animal Science 79: 301-308.

Carey H.V., Hayden U.L., and Tucker K.E. 1994. Fasting alters basal and stimulated ion transport in piglet jejunum. American Journal of Physiology-Regulatory, Integrative and Comparative Physiology 267: R156-R163.

English J.G.H. and Bilkei G. 2004. The effect of litter size and littermate weight on pre-weaning performance of low-birth-weight piglets that have been crossfostered. Animal Science 79: 439-443.

Hampson D.J. 1986. Alterations in piglet small intestinal structure at weaning. Research in veterinary science 40: 32-40. 
Hurley W.L. 2015. Composition of sow colostrum and milk. In: Farmer C. (ed.) The gestating and lactating sow. p 193-229.

Krugliak P., Hollander D., Schlaepfer C.C., Nguyen H., and MA T.Y. 1994. Mechanisms and sites of mannitol permeability of small and large intestine in the rat. Digestive Diseases and Sciences 39: 796-801.

Kuller W.I., van Beers-Schreurs H.M.G., Soede N.M., Langendijk P., Taverne M.A.M., Kemp B., and Verheijden J.H.M. 2007. Creep feed intake during lactation enhances net absorption in the small intestine after weaning. Livestock Science 108: 99-101.

Menard S., Cerf-Bensussan N., and Heyman M. 2010. Multiple facets of intestinal permeability and epithelial handling of dietary antigens. Mucosal immunology 3: $247-259$.

Meyer E. 2008. Influence of creep feeding during the suckling period on daily gain of piglets after weaning depending on body condition and feeding techniques. Zuchtungskunde 80: 224-232.

Miller H. and Slade R. 2003. Digestive physiology of the weaned pig. In: Pluske J., Le Dividich J., and Verstegen M. (eds.) Weaning the pig: concepts and consequences. Wageningen Academic Publishers, The Netherlands.

Moeser A.J., Klok C.V., Ryan K.A., Wooten J.G., Little D., Cook V.L., and Blikslager A.T. 2007. Stress signaling pathways activated by weaning mediate intestinal dysfunction in the pig. American Journal of Physiology Gastrointestinal and Liver Physiology 292: G173-G181.

Morgan C.A., Lawrence A.B., Chirnside J., and Deans L.A. 2001. Can information about solid food be transmitted from one piglet to another? Animal Science 73: 471-478.

Munsterhjelm C., Peltoniemi O.A.T., Heinonen M., Hälli O., Karhapää M., and Valros A. 2009. Experience of moderate bedding affects behaviour of growing pigs. Applied Animal Behaviour Science 118: 42-53.

Nicol C.J. and Pope S.J. 1994. Social learning in sibling pigs. Applied Animal Behaviour Science 40: 31-43. 
Oostindjer M., Bolhuis J.E., Mendl M., Held S., van den Brand H., and Kemp B. 2011. Learning how to eat like a pig: Effectiveness of mechanisms for vertical social learning in piglets. Animal Behaviour 82: 503-511.

Pajor E.A., Fraser D., and Kramer D.L. 1991. Consumption of solid food by suckling pigs: individual variation and relation to weight gain. Applied Animal Behaviour Science 32: 139-155.

Pluske J.R., Hampson D.J., and Williams I.H. 1997. Factors influencing the structure and function of the small intestine in the weaned pig: a review. Livestock production science 51: 215-236.

Price E.R., Brun A., Caviedes-Vidal E., and Karasov W.H. 2015. Digestive Adaptations of Aerial Lifestyles. Physiology 30: 69-78.

Sigalet D., Martin G., and Poole A. 2000. Differential sugar absorption as a marker for adaptation in short bowel syndrome. Journal of pediatric surgery 35: 661-664.

Spreeuwenberg M., Verdonk J., Gaskins H., and Verstegen M. 2001. Small intestine epithelial barrier function is compromised in pigs with low feed intake at weaning. The Journal of Nutrition 131: 1520-1527.

Sulabo R.C., Jacela J.Y., Tokach M.D., Dritz S.S., Goodband R.D., Derouchey J.M., and Nelssen J.L. 2010. Effects of lactation feed intake and creep feeding on sow and piglet performance. Journal of Animal Science 88: 3145-3153.

Turpin D.L., Langendijk P., Chen T.-Y., and Pluske J.R. 2016. Intermittent suckling in combination with an older weaning age improves growth, feed intake and aspects of gastrointestinal tract carbohydrate absorption in pigs after weaning. Animals 6: 66.

van Beers-Schreurs H.M., Nabuurs M.J., Vellenga L., Kalsbeek-van der Valk H.J., Wensing T., and Breukink H.J. 1998. Weaning and the weanling diet influence the villous height and crypt depth in the small intestine of pigs and alter the concentrations of short-chain fatty acids in the large intestine and blood. The Journal of nutrition 128: 947-953.

van Nieuwamerongen S.E., Soede N.M., van der Peet-Schwering C.M.C., Kemp B., and Bolhuis J.E. 2015. Development of piglets raised in a new multi-litter housing system vs. conventional single-litter housing until 9 weeks of age. Journal of Animal Science 93: 5442-5454. 
Vente-Spreeuwenberg M. and Beynen A. 2003. Diet-mediated modulation of small intestinal integrity in weaned piglets. In: Pluske J., Le Dividich J., and Verstegen M. (eds.) Weaning the pig: concepts and consequences. p 145-198.

Wijtten P.J., van der Meulen J., and Verstegen M.W. 2011. Intestinal barrier function and absorption in pigs after weaning: a review. British journal of nutrition 105: 967-981. 


\section{CHAPTER 5}

Post-weaning social and cognitive performance of piglets raised pre-weaning either in a complex multi-suckling group housing system or in a conventional system with a crated sow

S.E. van Nieuwamerongen ${ }^{1^{*}}$, M. Mendl ${ }^{2}$, S. Held ${ }^{2}$, N.M. Soede ${ }^{1}$, J.E. Bolhuis $^{1}$

${ }^{1}$ Adaptation Physiology Group, Department of Animal Sciences, Wageningen University \& Research, PO Box 338, 6700 AH Wageningen, The Netherlands;

${ }^{2}$ School of Veterinary Sciences, Animal Welfare and Behaviour Group, University of Bristol, UK.

${ }^{*}$ Corresponding author: S.E. van Nieuwamerongen at

Sofie.vannieuwamerongen@wur.nl.

Under revision at Animal Cognition (2017) 


\subsection{Abstract}

We studied the social and cognitive performance of piglets raised pre-weaning either in a conventional system with a sow in a farrowing crate (FC) or in a more complex multi-suckling (MS) system, in which a group of 5 sows and their piglets could interact in a more physically enriched and spacious environment. After weaning at 4 weeks of age, post-weaning housing consisted of pens bedded with sawdust, straw, and peat, containing 4 litter-mates each. From each pen, 1 pair consisting of a dominant and a submissive pig was selected, based on a feed competition test (FCT) 2 weeks post-weaning. This pair was used in an informed forager test (IFT) which measured aspects of spatial learning and foraging strategies in a competitive context. In short, during individual training, submissive (informed) pigs learned to remember a bait location in a testing arena with 8 buckets (the same bucket was baited in a search visit and a subsequent relocation visit), whereas dominant (noninformed) pigs always found the bait in a random bucket (search visits only). After learning their task, the informed pigs' individual search visit was followed by a pairwise relocation visit in which they were accompanied by the non-informed pig. MS and FC pigs showed few distinct behavioural differences, but there were some indications that FC pigs established clearer dominance relationships in the FCT. Informed MS and FC pigs performed similarly in learning their task in the IFT. MS and FC pigs, however, seemed to adopt different foraging strategies during the pairwise visits, as MS noninformed pigs seemed to make more use of the information of their informed partner.

Key words: informed forager test, social development, cognitive development, group housing, multi-suckling system, pigs 


\subsection{Introduction}

Rearing conditions can have a great impact on an animal's social and cognitive development. In conventional housing systems, piglets are reared in a farrowing pen with a crated sow, which provides an environment that is limited in stimuli that would promote piglet development; the sow is confined, which restricts sow-litter interaction and learning from the mother (Oostindjer et al., 2011), piglets have no contact with other litters, the environment is generally barren with minimal enrichment material and no rooting substrate, and the pen has a relatively simple design. This is in large contrast with the environment that a pig would encounter under natural conditions. In the farrowing season, wild boar live in family groups consisting of several sows and their offspring, in a complex and enriched environment (Gundlach, 1968; Meynhardt, 1980). Given the opportunity, domestic pigs will form a similar social structure and display similar natural behaviours as their ancestors (Jensen, 1986; Stolba and Wood-Gush, 1989; Petersen et al., 1990). Hence, the social and physical environment in a conventional system restricts expression of natural behaviours, which may be important for the development of domestic pigs.

We have developed an alternative farrowing system that better resembles the natural situation, with more space and social and physical enrichment than in a conventional system (for a previous version of the system, see van Nieuwamerongen et al., 2015). This multi-suckling (MS) system houses 5 sows together with their piglets and has 5 farrowing pens connected to a communal area, which is divided in areas for resting, eating, and dunging. This MS system has several properties that can affect piglets' social and cognitive development; a spacious environment with enrichment, possibilities for interactions with multiple sows, and pre-weaning mingling with piglets from other litters. An increased space allowance and a more complex pen design allow more possibilities for the development of a range of social skills, including avoidance and threatening behaviour (McGlone and Curtis, 1985; Weng et al., 1998), and play behaviour, which is thought to be important for social development (Spinka et al., 2001). Compared with single-litter housing, pre-weaning contact with multiple litters has been shown to reduce aggression after weaning towards familiar pigs (Hessel et al., 2006) and unfamiliar pigs (Kanaan et al., 2012), and to stimulate quicker formation of a stable 
dominance hierarchy post-weaning (D'Eath, 2005). The larger group size, resulting from pre-weaning mingling of sows and litters, can also affect social behaviour; pigs have been shown to display a lower level of aggression when housed in larger groups (Samarakone and Gonyou, 2009).

Indeed, pigs reared in an MS system showed less aggression when mixed with unfamiliar pigs after weaning (Li and Wang, 2011; Verdon et al., 2016) and established clearer dominance relationships among familiar pigs in a competitive situation, whilst also expressing less aggression (De Jonge et al., 1996). The latter study showed long-term effects of early social experiences, as differences between pigs from the different pre-weaning housing systems were found up until puberty. The larger group and more diverse group composition with multiple sows and litters in MS systems also provide more opportunities for social learning. In our MS system, social learning of eating behaviour is specifically stimulated by the use of a communal feeding area, where piglets can learn to eat solid food together with the sows and other piglets.

As the MS system provides a more complex social and physical environment for the piglets, it might be expected that their cognitive development (which includes aspects of memory, learning, problem solving, and decision-making) is more stimulated in the MS system than in a conventional system. It has been hypothesised that one of the functions of cognition is to enable an animal to deal with environmental complexity, which includes aspects of both the social and physical (i.e. non-social) environment (Godfrey-Smith, 2002). Specifically, the complexity of the social environment, related e.g. to group size, may be a driving force for the development of certain cognitive abilities, such as social learning (i.e. the acquisition of new skills, information or behaviour as result of interacting with other individuals) (Godfrey-Smith, 2002; Croney and Newberry, 2007; Arbilly et al., 2014). Several studies have compared aspects of cognitive performance between pigs reared under physically enriched or barren conditions in spatial tasks. Sneddon et al. (2000) found that pigs reared in enriched pens learned a spatial task quicker than pigs reared in barren pens, although de Jong et al. (2000) found no such difference. Furthermore, enrichment generally improved aspects of short term memory (e.g. working memory) and/or longer term memory (e.g. reference memory) (de Jong et al., 2000; Bolhuis et al., 2013; Grimberg-Henrici et al., 2016), although Jansen et al. (2009) found no difference between enriched and barren pigs in finding an 
alternative route to exit a maze and subsequently remembering the detour with a 1-day interval. Although not found consistently, it appears that an enriched environment may have beneficial effects on aspects of cognitive development.

Given that pigs reared in an MS system have experienced more social and physical environmental complexity than pigs reared in a conventional farrowing system, we hypothesised that MS pigs would be better prepared to deal with social and cognitive challenges later in life. We measured this using a feed competition test and a so-called informed forager test (IFT) after weaning. The IFT measures aspects of spatial learning and foraging strategies in a competitive context (Held et al., 2000). Two foraging roles have been described in several group foraging species: producers, which localise food sources autonomously; and scroungers, which eat from producers' findings (Giraldeau and Lefebvre, 1986; Beauchamp and Giraldeau, 1997). When food is distributed in patches, as is the case for wild boar (Meynhardt, 1980), the most dominant animals within a group can benefit from exploiting submissive producers, whereas the latter may best forage alone or find tactics to avoid exploitation (Held et al., 2000). Previous studies have shown that domestic pigs can flexibly adapt their behaviour to optimise their foraging strategy, depending on the type of food reward and the behaviour of other pigs (Held et al., 2000, 2002; Held et al., 2005; Held et al., 2010). In the IFT, pigs are directed to adopt a certain forager role, by training pairs consisting of a submissive 'informed' pig, which has knowledge about the location of a food reward in an arena with hidden buckets, and a dominant 'non-informed' pig, which is unaware of the reward's location. In phase 1 of the IFT, both pigs are trained to be producers, as they have to find the reward individually. Informed (I) pigs, however, learn that the location of the reward is always the same in 2 successive visits to the arena, whereas non-informed (NI) pigs learn that the reward is to be found in a non-predictable random location during each visit. In phase 2, the I and NI pig are tested in pairs, after the individual search visit of the I pig. Thus, in phase 2, I pigs are informed about the location of the reward, and NI pigs have the opportunity to switch to a scrounger role by following and displacing the I pig from the reward. Subsequently, I pigs may also alter their foraging strategy to minimise exploitation (Held et al., 2002).

We hypothesised that during the selection of pairs of dominant (NI) and submissive (I) pigs, MS pairs would show less aggression and a clearer 
dominance relationship than the conventionally reared control pigs. Furthermore, we expected MS pigs to perform better than control pigs during the IFT. In other words, I pigs would learn their task faster, both I and NI pigs would demonstrate better working memory regarding food locations they have already visited, and NI pigs would benefit more from the knowledge of I pigs, if they have been raised in the MS system compared with control conditions.

\subsection{Materials and methods}

\subsubsection{Animals and housing}

The experiment was approved by the Animal Care and Use Committee of Wageningen University. In total, 64 piglets (Tempo x Topigs 20) were studied, equally distributed over 2 successive batches. Before weaning, piglets were housed at the animal facilities of Swine Innovation Centre Sterksel, the Netherlands, either in a multi-suckling (MS) system consisting of 5 sows and their litters or with a sow in a conventional farrowing crate (FC). All sows were multiparous and allocation of the sows to the pre-weaning housing treatments was balanced for parity. In each batch, 4 healthy litter-mates from 4 litters were selected per system to participate in this study. Per litter, 1 light and 1 heavy piglet from both sexes were selected based on body weight 6 days before weaning (day -6) and taking into account a similar relative weight difference per pre-weaning housing treatment. Before selection, mean litter weights at day -6 were $6.39 \pm 0.30 \mathrm{~kg}$ in the MS system and $6.16 \pm 0.34 \mathrm{~kg}$ in the FC system. Body weight of the selected piglets was $7.53 \pm 0.23 \mathrm{~kg}$ for the 'heavy' MS piglets, $5.57 \pm 0.23 \mathrm{~kg}$ for the 'light' MS piglets, $7.34 \pm 0.23 \mathrm{~kg}$ for the 'heavy' FC piglets, and $5.44 \pm 0.20 \mathrm{~kg}$ for the 'light' FC piglets. After weaning at $27.1 \pm 0.4$ days of age, the piglets were transported to the research facility 'Carus' of Wageningen University \& Research, the Netherlands. Postweaning housing conditions (see below) were the same for all piglets.

\subsubsection{General pre-weaning management}

Piglets were ear tagged and weighed within 24 hours post-partum (p.p.). Litter sizes were standardised between 24 to 48 hours p.p. according to the number of functional teats available per sow. Piglets were tail docked and received an iron injection within 4 days after birth. Male piglets were not castrated. Pre-starter creep feed (11.6 MJ of NE, 17.5\% crude protein, and 1.17\% ileal digestible 
lysine) was provided to the piglets in round feeders (diameter $28 \mathrm{~cm}$ ) twice daily from day 12 . On days 21 and 22 pre-starter feed was gradually mixed with weaner feed (9.9 MJ of NE, 16.0\% crude protein, and $0.99 \%$ ileal digestible lysine), and after day 22 only weaner feed was provided. Water was available ad libitum. Animal health was checked twice daily.

\subsubsection{Pre-weaning housing}

\section{Multi-suckling system}

MS housing consisted of 5 farrowing pens connected to a communal area (Figure 5.1). Sows could access all areas from the moment of entry in the system. The farrowing pens were $3.2 \times 2.2 \mathrm{~m}$ each and contained a feed trough for the sow, a water nipple for the piglets, and a covered piglet nest of $0.7 \times 1.6$ $\mathrm{m}$ with heated solid flooring. The floor in the rest of the pen consisted of solid concrete and concrete slats. Five hessian sacks were provided per pen as nesting material. From day 2 p.p. onward, 2 handfuls of long-stemmed straw were provided daily per farrowing pen. The adjacent communal area was divided in an area for feeding (solid concrete and metal slats),

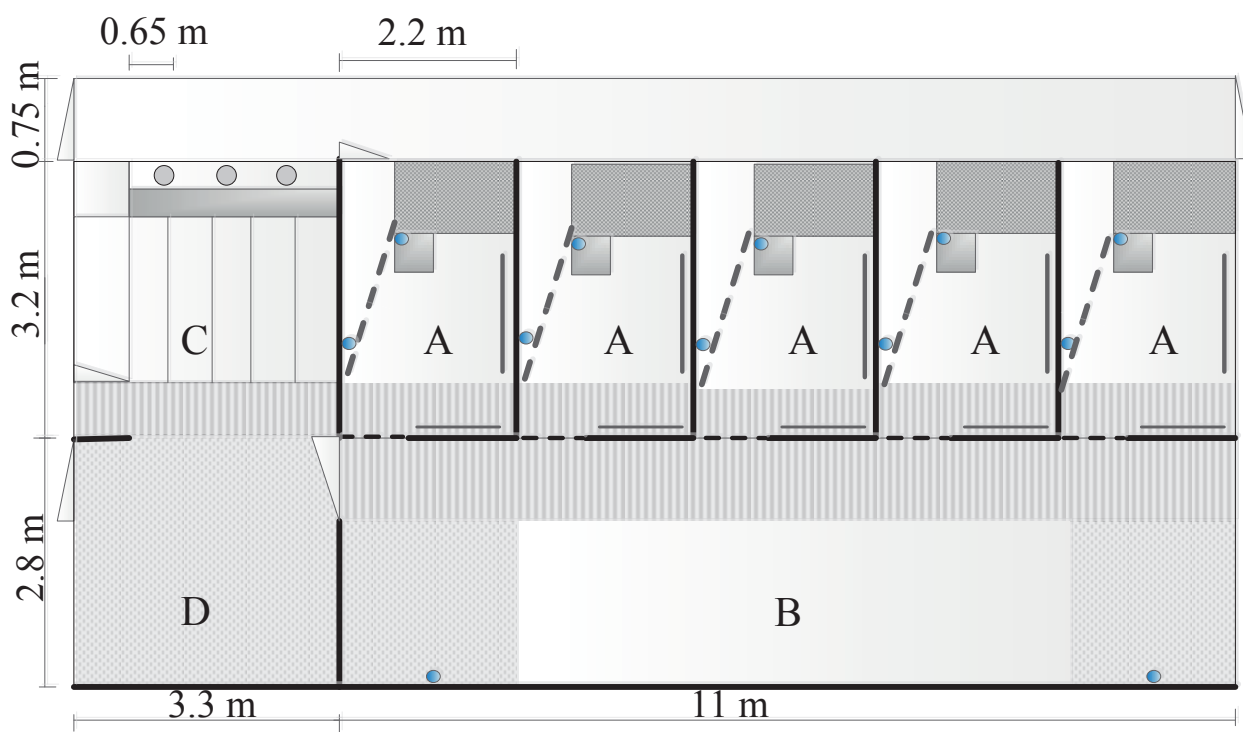

Figure 5.1. Layout of the multi-suckling system. The system has 5 farrowing pens (A with piglet nest, sow feeder, drinkers, and anti-crushing devices) connected to a communal area with a lying area (B - with 2 drinkers), feeding area ( $\mathrm{C}$ - with 5 sow feeding places surrounded by a piglet area with piglet feeders), and a dunging area (D). 
defecating/urinating (metal slats), and lying (solid concrete and metal slats). Five hessian sacks and 5 ropes were attached to the partitions surrounding the resting area and were replaced if needed. The communal feeding area contained 5 feeding places for the sows separated by horizontal metal bars, and a surrounding area with piglet feeders accessible to only the piglets. Each individual sow was locked in her own farrowing pen daily between 16:30 to 07:30 $\mathrm{h}$ until farrowing of that particular sow had ended. From that moment onward, sows could freely access all farrowing pens and communal areas. Piglets were given access to the whole system at a mean age of $7.9 \pm 0.3$ days. This was achieved by removing a $31 \mathrm{~cm}$ high piglet barrier at the entrance from each farrowing pen. In addition, a 'piglet door' $(0.4 \times 0.3 \mathrm{~m})$ was removed from the front wall of each farrowing pen, to provide piglets more space to move in and out of the farrowing pens. The sows were floor fed twice daily in the communal area and piglets could eat together with the sows from the sow feed, and from creep feed provided in the piglet feeders. Sows were fed in their own farrowing pen only in the afternoons before farrowing and in the first days after farrowing in case the sows did not leave their pen to eat in the communal area.

\section{Conventional system}

Each FC litter was kept in a pen of $2.4 \times 1.8 \mathrm{~m}$, which contained a farrowing crate of $2.0 \times 0.7 \mathrm{~m}$ for the sow. The pens were situated in farrowing units containing 12 pens each. The floor consisted of metal slats within the crate, a solid floor of $1.2 \times 0.3 \mathrm{~m}$ with a heat lamp for the piglets, and plastic slats in the remaining area. The sow had a feed trough and a drinking nipple available. The piglets had access to a separate drinking nipple and creep feed was provided in a round feeder located in the corner at the posterior side of the sow. One day prior to expected farrowing, sows received a hessian sack as nesting material. During the whole pre-weaning period, a plastic roll was available for both sow and piglets, and a chain with a wooden block was available as enrichment for the sow.

\subsubsection{Post-weaning housing}

After weaning, 32 piglets per batch were transported (for about 1.5 hours) to their new housing facilities. During transport, the $16 \mathrm{MS}$ piglets were penned in 1 group, whereas the $16 \mathrm{FC}$ piglets were penned in groups of 4 litter-mates. 
After arrival, MS and FC litters were equally divided over 2 adjacent rooms, where the 4 pens in 1 room were filled alternatingly with piglets from MS and FC litters. Each pen $(2.7 \times 3.6 \mathrm{~m})$ housed 4 litter-mates and was bedded with $550 \mathrm{~L}$ of sawdust, $10 \mathrm{~kg}$ of straw, and $90 \mathrm{~L}$ of peat on a solid floor. On a daily basis, soiled bedding was removed from the pens and $70 \mathrm{~L}$ of fresh sawdust and $1 \mathrm{~kg}$ of straw was added. Fresh peat was added on a weekly basis (45 L). A hessian sack, a rope, and a chain with bolts were available as further enrichment. The same weaner feed that was provided in the late pre-weaning phase was offered ad libitum in a feeder with 4 eating places. Two weeks after weaning, a starter diet (9.6 MJ of NE, 17.3\% crude protein, and 1.04\% ileal digestible lysine) was provided in a new feeder with 1 eating place. A grower diet was provided from 5 weeks post-weaning onwards (9.6 MJ of NE, 16.3\% crude protein, and $0.93 \%$ ileal digestible lysine). Water was continuously available from a drinking nipple. Animal health was checked twice daily. All pigs were marked with a number on their back using stock marker spray to allow individual identification.

\subsubsection{Behaviour tests}

\section{Feed competition test (FCT)}

The feed competition test (FCT) took place on days 14 and 15 post-weaning. Piglets were feed deprived by removing the feeders from the pens in the afternoons before the tests (around 16:30 h). All 4 possible combinations of a 'heavy' vs. a 'light' piglet were tested per pen. Tests were divided over 2 mornings and 2 pairs of piglets were tested per pen before proceeding to the next pen. Each piglet was tested only once per morning. The order of testing pens was the same for both testing days and was balanced for pre-weaning housing treatment. Before a pair was tested, all 4 piglets were removed from their pen and led into the corridor adjacent to the pens. A bucket attached on top of 2 wooden boards arranged in a cross (to prevent piglets from knocking over the bucket) was placed where the feeder was normally located. The boards were covered with bedding material to prevent piglets from slipping on the boards. The bucket was filled with $100 \mathrm{~g}$ of feed. The pair to be tested was separated from the other 2 litter-mates using a board and was led back into their pen. The test started when the first pig had completely entered the pen and lasted for 4 minutes. The behaviours listed in Table 5.1 were recorded by 2 
Table 5.1. Ethogram used in the feed competition test and informed forager test.

\begin{tabular}{ll}
\hline Measurement & Description \\
\hline Bucket access (duration): & Piglet has its head in the bucket and has exclusive access to the bucket. \\
Head knock (frequency): & $\begin{array}{l}\text { Piglet gives a single horizontal or vertical knock with the head or a forward } \\
\text { thrust with the snout toward the other piglet, without biting. }\end{array}$ \\
Bite (frequency): & $\begin{array}{l}\text { Piglet gives a single bite (snapping jaws) at the other piglet. Can be } \\
\text { performed while giving a head knock. }\end{array}$ \\
$\begin{array}{l}\text { Push (frequency): } \\
\text { Piglet exerts force with the body to the other piglet's body (without } \\
\text { displacing the other piglet). }\end{array}$ \\
$\begin{array}{l}\text { Piglet gains exclusive access to the bucket by pushing the other piglet away } \\
\text { from the bucket while the other piglet had bucket access. }\end{array}$
\end{tabular}

observers. After the test, all 4 piglets were led back into their pen. The feeders were put back in the pens after all tests of 1 morning were completed. Based on the performance in the FCT, 1 pair per pen was selected to participate in the informed forager test, with the dominant pig being trained as the non-informed pig and the submissive pig being trained as the informed pig.

\section{Informed forager test (IFT)}

The IFT was carried out from 5 to 15 weeks of age and consisted of 2 phases, based on Held et al. (2000). In short, after a habituation period, individual piglets were trained in phase 1 to locate 1 baited bucket in a testing arena (see Figure 5.2). Informed (I) pigs were the submissive pigs within each pair and were allowed to search for the bait in the testing arena twice (i.e. 1 search visit and 1 relocation visit) within a trial, with the bait located in the same bucket in both visits of the trial. I pigs were thus trained to remember the specific location of a baited bucket after a search visit and use this information in a subsequent relocation visit. The non-informed (NI) pigs were the dominant pigs within each pair and were trained to search for the baited bucket, without having prior knowledge about the location of the bait in the testing arena (i.e. NI pigs had only search visits). In phase 2, I pigs were accompanied by their NI pen-mate during the relocation visit.

\section{Habituation to the IFT}

Before starting the IFT, the piglets were gradually habituated to elements of the test over a course of 13 days when they were 5 to 7 weeks old. The piglets were sequentially habituated to: being in the presence of people in their home pen, 
touching buckets with bait in their home pen, visiting the testing room with all 4 pen-mates and with all 8 buckets baited, visiting the testing room individually with 8 baited buckets, visiting the testing room individually with 4 baited buckets, visiting the testing room individually with 2 baited buckets and, finally, visiting the testing room individually with 2 baited buckets and all buckets covered with chopped straw. Piglets were exposed to the testing room with only 1 baited bucket for the first time during testing.

\section{General procedure for IFT phase 1 and 2}

The IFT took place between 8 to 15 weeks of age. Two trials were run daily from Monday to Friday between 08:30 and 13:30 h. Pigs were feed deprived by closing the feeders around 16:30 $\mathrm{h}$ before each testing day. The metal buckets in the testing room all contained a removable metal grid,

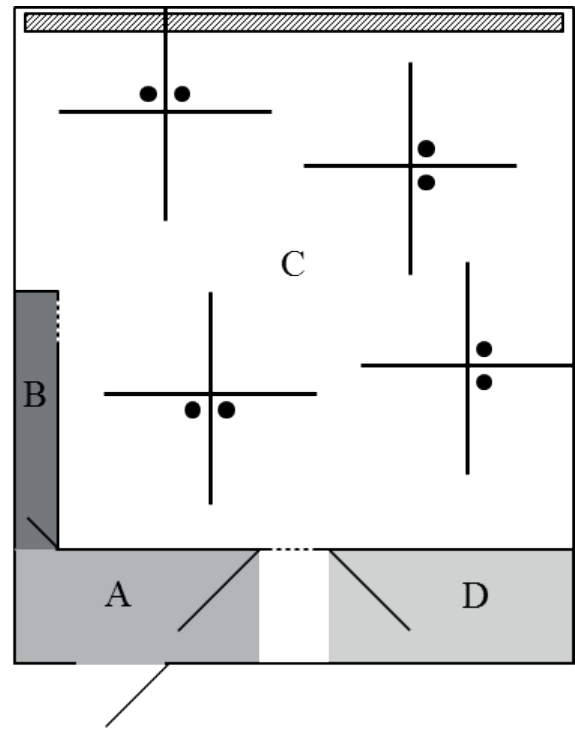

Figure 5.2. Layout of the testing room $(7.4 \times 6.3 \mathrm{~m})$ used for the informed forager test. $\mathrm{A}=$ the area where the pigs entered the testing room, $\mathrm{B}=$ the start box, $\mathrm{C}=$ the testing arena, which contained 4 crosses (60 $\mathrm{cm}$ high) with 2 buckets per cross, and $\mathrm{D}=$ the waiting area for the litter-mates that did not participate in the test, containing 2 toys and a hessian sack. Dotted lines represent guillotine doors and angled solid lines represent hinged doors. The dashed area in the back represents a drainage area. creating a double bottom under which bait (4 chocolate raisins) could be placed. Before pigs entered the testing arena, bait was placed under the double bottom of each bucket, which equalised the odour cues from each bucket, but prevented pigs from accessing the bait. All double bottoms were covered with chopped straw (to hide bait from direct sight and increase searching time) and 1 bucket was filled with bait that was placed within the chopped straw and was thus accessible to the pigs. The location of this baited bucket was randomised for every subsequent trial. For each trial, the 4 pigs from 1 pen were guided to the testing room. One trial for an NI pig consisted of a search visit. One trial for an I pig consisted of a search visit followed immediately by a relocation visit in which the same bucket was 
baited. The test started when the pig (or first pig in paired visits) had completely entered the arena and the test ended when the pig (or last eating pig in paired visits) had lifted its head from the baited bucket. An auditory cue and the opening of the exit door (between A and D in Figure 5.2) then followed, after which the pig(s) left the testing arena. The maximum testing duration per visit was 3 minutes. If the bait had not been found within this time, an experimenter stepped in the testing arena and showed the location of the bait, by walking to the baited bucket and enticing the pig to follow. When the pig(s) had left the testing arena, leftover bait was quantified and removed, the straw of the bucket that was baited was replaced, and new bait was placed. If needed, faeces and urine were removed from the testing arena. After testing both the I and NI pig, they returned together with their 2 pen-mates to their home pen where they received 4 handfuls of feed on the floor after each trial. If needed, faeces and urine were removed from the testing arena, start box, and waiting area, before bringing pigs from the next pen to be tested to the testing room. All pens were tested during the first series of trials, before starting the second series of trials. Pens were tested in the same order during the first and second series of trials. The testing order per day was randomised and balanced for pre-weaning housing treatment. After the pigs of a pen finished the second trial, the feeder in their home pen was opened again. After each testing day, the whole testing room was cleaned with water and all-purpose cleaner.

During phase 2 in batch 1, few interactions between the I and NI pigs occurred, and therefore the procedure and the bait used for the paired visits were adjusted for batch 2 . The bait was changed to 4 chocolate raisins and $25 \mathrm{~g}$ of feed in the individual visits (this was also the bait that was placed under the double bottoms) and 6 chocolate raisins and $50 \mathrm{~g}$ of feed in the paired visits. In addition, NI pigs were given more opportunity to discover that there was still a baited bucket to be found in phase 2 (and not only in phase 1). This was done by using a minimum testing duration of 2 minutes (while still maintaining the maximum testing time of 3 minutes), only in case the I pig found the bait without the NI pig being present near the baited bucket (i.e. in the imaginary square around the bucket that was formed by the crosses).

\section{IFT phase 1 - individual training}

The I pig was guided to the start box while its pen-mates remained in the 
waiting area. As soon as the door to the testing arena was opened, the I pig was allowed to search for the bait. After finding the bait, the I pig was guided via area A (see Figure 5.2) to the start box again for a relocation visit in which the bait was placed in the same bucket. After completing the relocation visit, the I pig was guided to the waiting area. Thereafter, the NI pig was released into the testing arena and was allowed to search for the bait that was placed in a different randomised location. The frequency, duration, and latency of visits to all buckets were scored by 1 observer. The difference between search and relocation visits in the number of bucket visits, revisits to buckets already inspected, and the latency to find the bait was used to indicate whether I pigs learned their task. Moreover, perseverance errors were scored to test the tendency of I pigs to search for the bait in the same location in the 2 successive trials of 1 day (as they were trained to relocate the same baited bucket within a trial and search for the bait in a different location in the next trial). A perseverance error occurred on days where an I pig, during the second trial of the day, first visited the bucket that was baited in the first trial of that day. Perseverance errors were not taken into account for NI pigs, as they always found the bait in a random location. For both I and NI pigs, revisits to buckets were assessed as a measure of impaired working memory (van der Staay et al., 2012). Pairs of I/NI pigs proceeded to phase 2 when the I pig reached the criterion of visiting a maximum of 2 unbaited buckets during the relocation visits in at least 6 out of 8 consecutive trials (Held et al., 2005; Held et al., 2010).

\section{IFT phase 2-pairwise testing}

After individual training, I pigs were tested together with their NI pen-mates. The search visit of the I pigs was executed as in phase 1. During the relocation visit, however, the I pig was accompanied by its NI pen-mate. The frequency, duration, and latency of visits to all buckets, and the interactions between the 2 pigs were scored by 2 observers, with each observer scoring 1 of the 2 pigs (see Table 5.1).

The differences between phase 1 and 2 in the number of bucket visits, revisits, and the latency to reach the first bucket and the baited bucket were used as indicators of the I and NI pigs' response to individual visits vs. paired visits. Moreover, the number of I-NI visits, where the NI pig visited the bucket that 
the I pig investigated immediately before, was used to determine in which trials 'close following' of the I pig by the NI pig occurred. The order of bucket visits was used as a criterion for I-NI visits (without restriction by a maximum time interval between bucket visits), consistent with the protocol of Held et al. (2000). Trials with 'close following' were those in which the number of I-NI visits divided by the total number of bucket visits of the NI pig was $\geq 0.5$ (Held et al., 2000), and were used to indicate if NI pigs made use of the information of the I pigs (in case an NI pig did not visit any buckets within a trial, the proportion of I-NI visits was set to 0 ). The number of displacements of the NI pig towards the I pig was used to indicate whether the NI pigs exploited the I pigs (Held et al., 2000).

\subsubsection{Statistical analysis}

Data were analysed with SAS 9.2 (SAS Inst. Inc., Cary, NC). Residuals were checked for normality and variables were transformed with a square root if needed. Results are presented as means \pm SEM. $P$-values below 0.05 were considered statistically significant.

\section{Feed competition test (FCT)}

Variables of the FCT were analysed using mixed models with pre-weaning housing treatment and batch as fixed effects, and a random effect of pen. The relative weight difference within the pair influenced total frequency of aggressive behaviours and tended to influence the number of displacements, and was therefore added to the model as a covariate. For the I-NI pairs selected per pen, differences in behaviour and body weight between NI and I pigs, and the effect of pre-weaning housing on these differences, were analysed in a mixed model with pre-weaning housing treatment, status (I or NI), housing treatment $\mathrm{x}$ status interaction, and batch as fixed effects, and a random effect of pen.

\section{Informed forager test (IFT) phase 1}

The number of trials run during phase 1 ranged from 18 to 31 per pen. As all pens completed at least 18 trials in phase 1 , these first 18 trials per pen were analysed for treatment effects during individual training (when including all trials of phase 1, instead of only the first 18, similar results were found). During 
$24.2 \%$ of all visits in these 18 trials, the maximum testing time elapsed and pigs were guided to the baited bucket. The occurrence of these guided visits did not differ significantly between MS (27.3\%) and FC (21.1\%) pigs and were included in analyses. Results were similar whether these visits were included or not, unless indicated otherwise in the results.

The difference in number of bucket visits, revisits, and latency to reach the baited bucket between search and relocation visits of the I pigs, and the effect of treatment on these differences, were analysed using mixed models with preweaning housing treatment, visit type (search or relocation visit), housing treatment $\mathrm{x}$ visit type interaction, and batch. Repeated observations on the same individuals were taken into account by including a repeated effect of visit type at pen level.

To investigate the learning curve of the I pigs during relocation visits over time, the number of bucket visits, revisits, and latency to reach the baited bucket were analysed using the 18 trials averaged per testing day (i.e. 9 testing days were taken into account in the analyses). Mixed models were used, including pre-weaning housing treatment, testing day, housing treatment $\mathrm{x}$ day interaction, and batch. Repeated observations on the same individuals were taken into account by including a repeated effect of testing day at pen level.

The percentage of days during which a perseverance error occurred and the number of trials needed to reach the criterion to proceed to phase 2 were analysed with mixed models including pre-weaning housing treatment and batch as fixed effects. The same model was used to analyse the number of bucket visits, revisits, the latency to reach the first bucket, and the latency to reach the baited bucket for the NI pigs.

\section{Comparisons between IFT phase 1 and 2}

The number of trials run for phase 2 varied between 28 and 41 trials per pen. For the comparison between both phases of the IFT, the last 8 trials of phase 1 of a particular pair (in which I pigs had all reached the criterion) and the first 28 trials of phase 2 of a particular pair were analysed. The number of bucket visits, revisits, the latency to reach the first bucket, and the latency to reach the baited bucket were analysed with mixed models including pre-weaning housing treatment, phase, batch, and their 2-way and 3-way interactions. Batch was 
included in the interactions because the bait and testing procedure during phase 2 differed in batch 1 and 2. Repeated observations were taken into account by including a repeated effect of phase at pen level.

\section{IFT phase 2}

The proportion of I-NI visits and closely followed trials were analysed with generalised linear mixed models, including pre-weaning housing treatment, batch, and their interaction. The percentage of trials in which a pig ate from the bait was analysed with a generalised linear mixed model including status (I vs. NI) as a fixed effect, and pen within treatment and batch as a random effect. The generalised linear mixed models had a binomial distribution and logit link function. The occurrence of displacements was analysed with a Fisher's exact test, as MS pairs did not show any displacements in batch 1. Because no interactions can be analysed in the Fisher's exact test, both pre-weaning housing treatments were compared within each batch, and both batches were compared within each pre-weaning housing treatment. Relations between the percentage of trials in which I and NI pigs ate from the bait and the behaviour of both pigs were analysed with Spearman correlations, using averages per pen over the first 28 trials of phase 2 .

\subsection{Results}

\subsubsection{FCT}

When considering all heavy vs. light pig combinations ( 4 per pen), the total frequency of aggressive behaviours and the absolute difference in aggression between the 2 pigs tested was not affected by pre-weaning housing treatment (data not shown). In addition, the overall duration of bucket access, the difference within a dyad in the duration of bucket access, and the total number of bucket access bouts did not differ between MS and FC pigs. However, the difference within dyads in the number of bucket access bouts was smaller for MS pigs than for FC pigs $(0.8 \pm 0.2$ vs. $1.8 \pm 0.3, P<0.05)$, i.e. in MS pairs, pigs changed access to the bucket less often.

From each pen, 1 dyad was selected to represent a dominant and a submissive pig. From these selected I-NI pairs, the NI pig was on average $27.4 \%$ heavier (NI: $12.1 \pm 0.5 \mathrm{~kg}, \mathrm{I}: 9.5 \pm 0.4 \mathrm{~kg}, P<0.0001$ ) than the I pig and successfully 
displaced the other pig to get to the feed 3.3 times more often (NI: $3.9 \pm 0.5$, I: $1.2 \pm 0.4, P<0.0001)$. Moreover, during the 4-minute test, the NI pigs had 2.4 times longer access to the bucket with feed than the I pigs (NI: $156.6 \pm 10.2 \mathrm{~s}$, I: $65.8 \pm 6.5 \mathrm{~s}, P<0.0001)$. These differences between NI and I pigs were not affected by pre-weaning housing conditions. Additionally, FC non-informed pigs performed more head knocks, bites and pushes than FC informed pigs (13.9 \pm 2.7 vs. $6.6 \pm 1.5)$, whereas MS non-informed and informed pigs showed a similar frequency of these aggressive behaviours (MS informed: $8.5 \pm$ 1.9, MS non-informed $8.5 \pm 2.0$, treatment $\mathrm{x}$ status interaction $P<0.05$ ).

\subsubsection{IFT phase 1}

\section{I pigs}

In phase 1 of the IFT, I pigs were trained to remember the specific location of a baited bucket after a search visit and a subsequent relocation visit. The retention interval, i.e. the time between the start of the search visit and of the relocation visit, was $4 \pm 1$ minutes (average \pm SD). The effects of pre-weaning housing conditions and differences in performance between search and relocation visits for I pigs over the first 9 testing days (18 trials) are summarised in Table 5.2. During the relocation visits, I pigs visited fewer buckets $(3.4 \pm 0.2$ vs. $5.4 \pm 0.2)$, had fewer revisits to buckets that were already investigated ( 0.2 \pm 0.04 vs. $0.8 \pm 0.09$ ), and had a shorter latency to reach the baited bucket than in the search visits $(59.7 \pm 4.4$ vs. $97.8 \pm 7.0$ s). Pre-weaning housing did not affect these differences between search and relocation visits of the I pigs.

The percentage of days on which I pigs made a perseverance error (MS: $23.8 \pm$ 4.7, FC: $23.4 \pm 3.1$ ) and the number of trials needed to reach the criterion to proceed to phase 2 of the IFT (MS: $20.5 \pm 2.8$, range 11 to 31 ; FC: $17.4 \pm 2.0$, range 8 to 25) did not differ between pre-weaning housing treatments. During relocation visits of I pigs, the latency to reach the baited bucket decreased over the first 9 testing days (Figure 5.3), but there was no day effect on the number of bucket visits and revisits (data not shown). When omitting the guided visits (i.e. $24.2 \%$ of all visits in the first 18 trials), the day effect on latency to reach the bait became non-significant. 


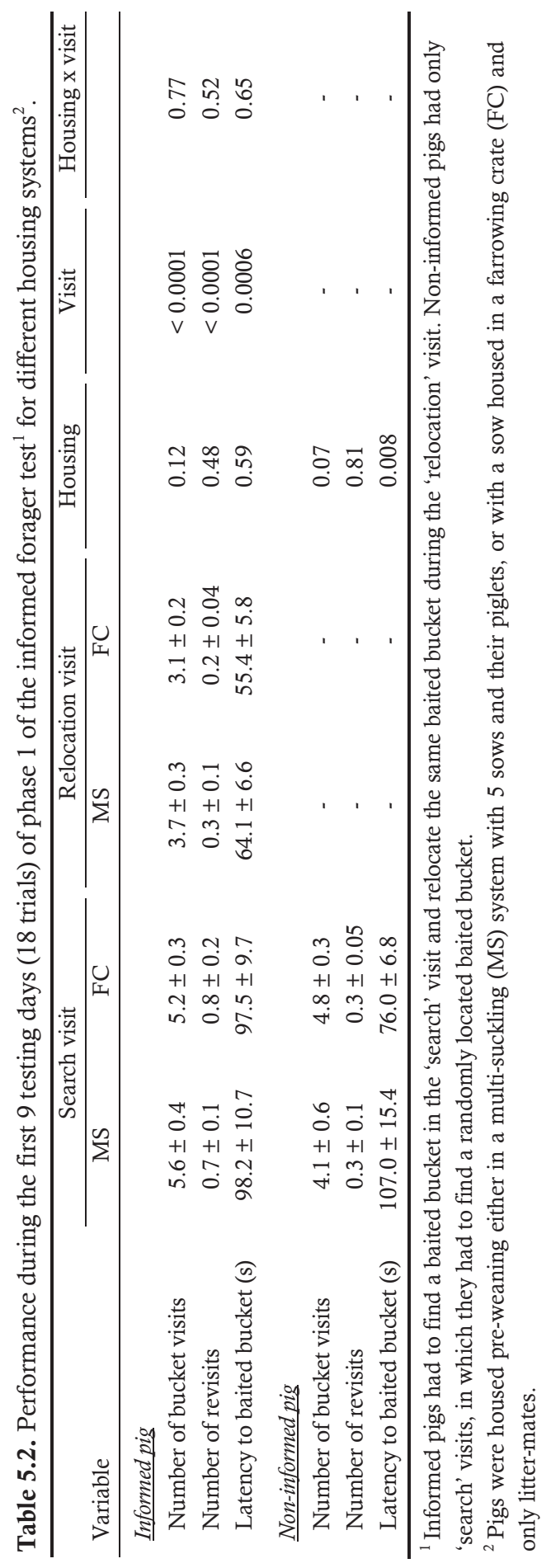




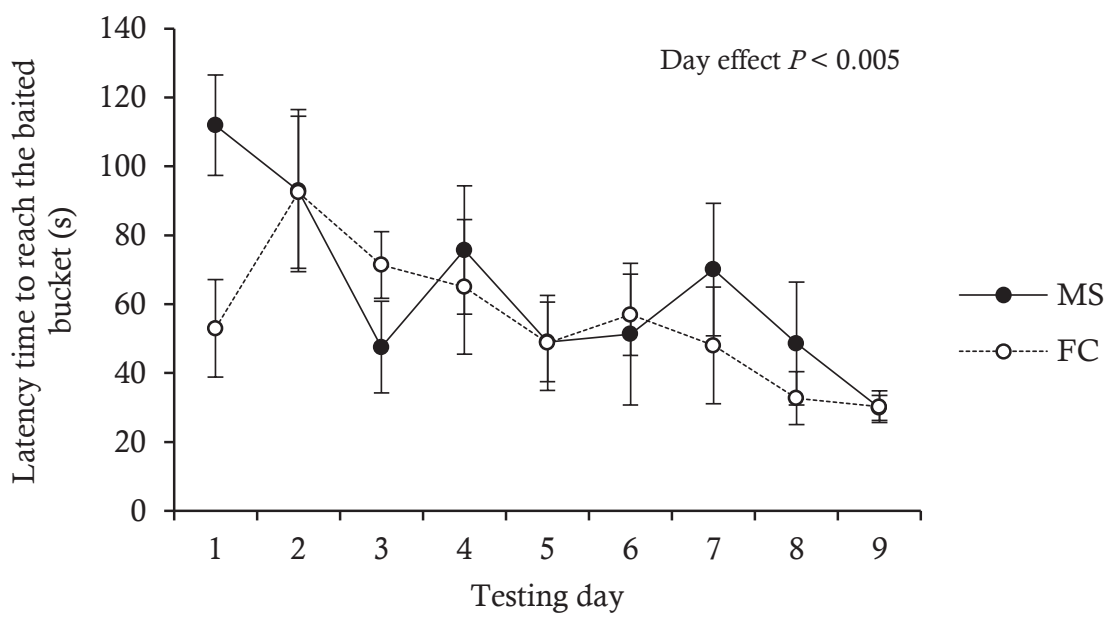

Figure 5.3. The latency to reach the baited bucket for informed pigs during the relocation visits of the first 9 testing days (18 trials) of phase 1 of the informed forager test. The informed pigs had to find a baited bucket in the 'search' visit and relocate the same baited bucket during the 'relocation' visit. Pigs were housed preweaning either in a multi-suckling (MS) system with 5 sows and their piglets, or with a sow housed in a farrowing crate (FC) and only litter-mates.

\section{NI pigs}

Non-informed MS pigs tended to visit fewer buckets and had a longer latency to reach the baited bucket than non-informed FC pigs during the search visits over the first 9 test days (18 trials). The number of revisits did not differ between non-informed MS and FC pigs (Table 5.2).

\subsubsection{IFT phase 2}

\section{I pigs}

During the paired visits in phase 2, I pigs tended to have a longer latency to visit the first bucket $(15.3 \pm 1.5$ vs. $11.0 \pm 1.2 \mathrm{~s})$, visited fewer buckets $(1.9 \pm$ 0.1 vs. $2.5 \pm 0.1)$, and tended to have fewer revisits $(0.01 \pm 0.01$ vs. $0.11 \pm$ 0.05 ) than during the individual visits in phase 1 (Table 5.3). Additionally, informed MS pigs visited more buckets than informed FC pigs during phase 1 and 2 combined $(2.4 \pm 0.1$ vs. $2.0 \pm 0.1)$. 


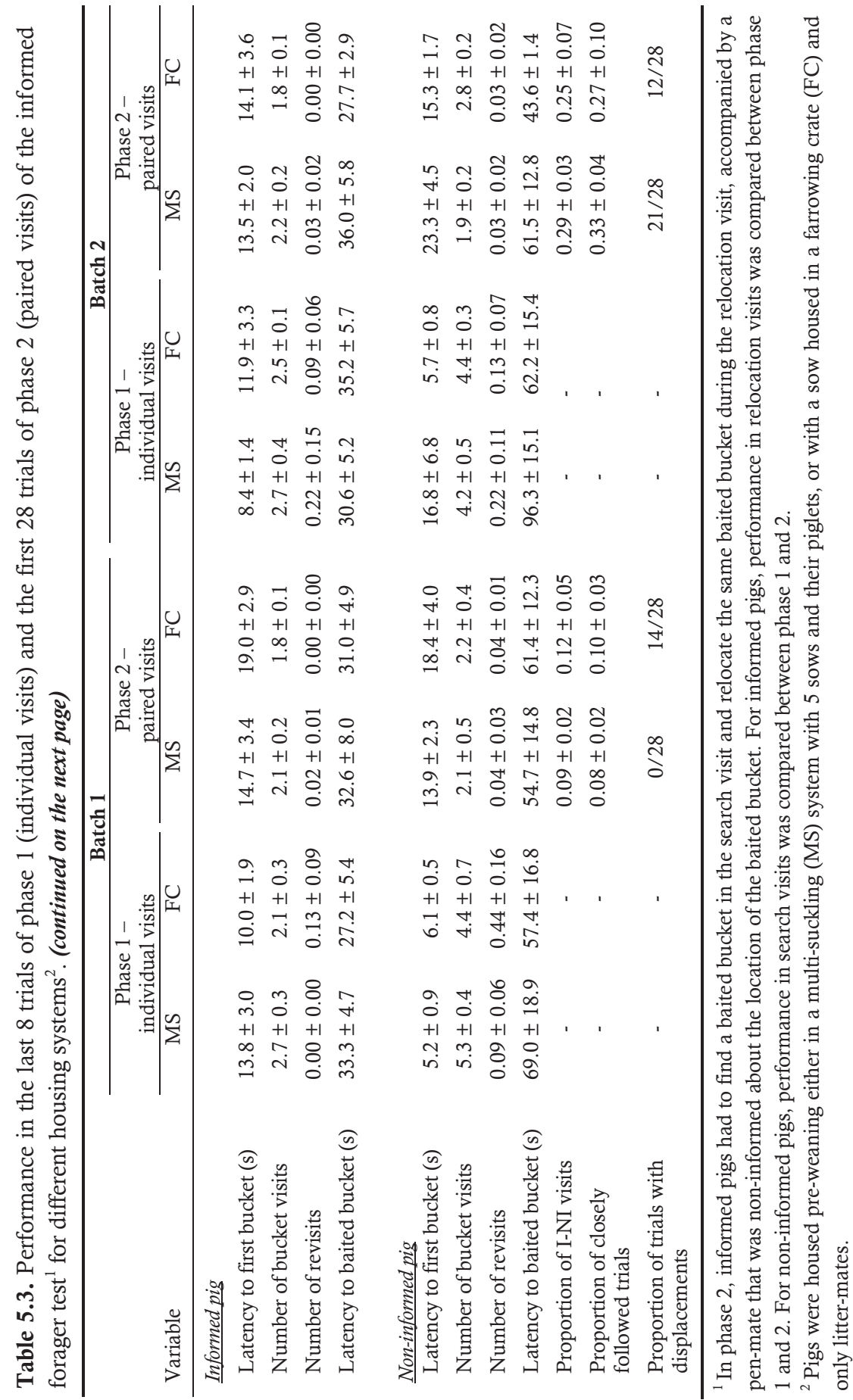




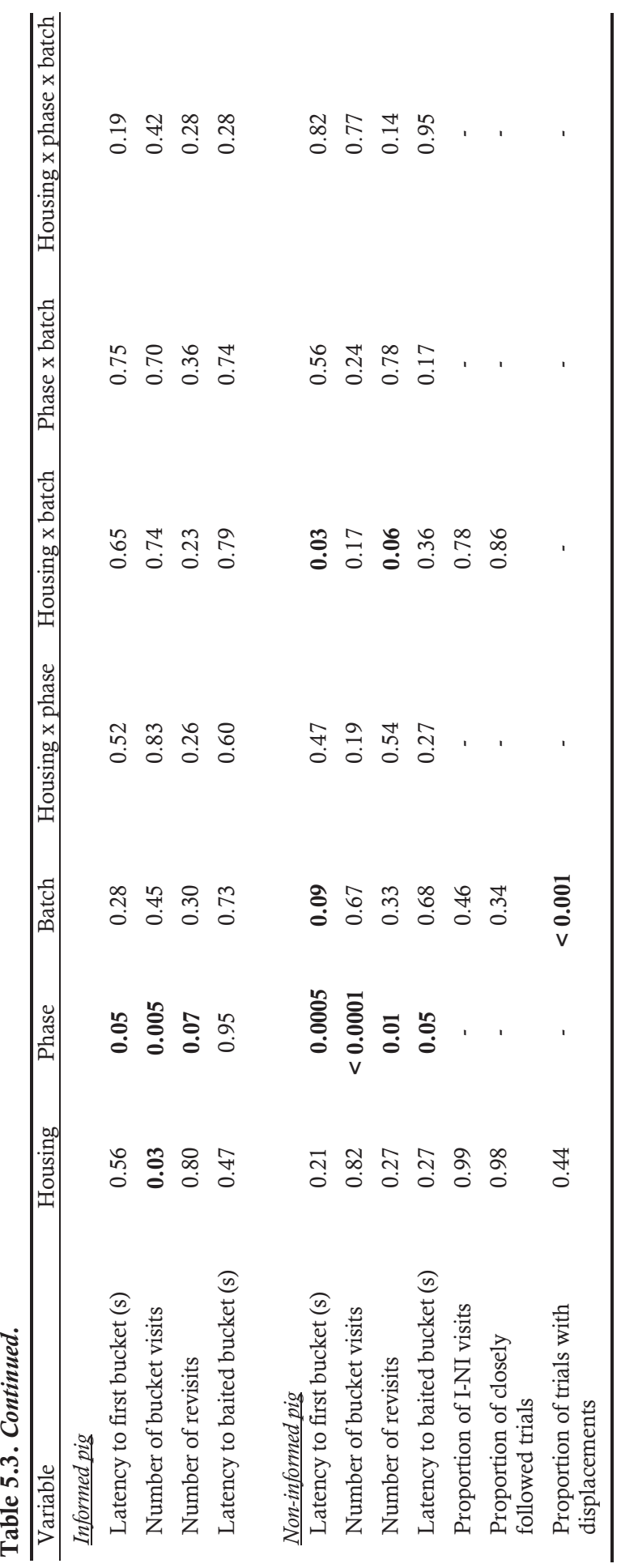




\section{NI pigs}

During the paired visits in phase 2, NI pigs had a longer latency to visit the first bucket $(17.7 \pm 1.8$ vs. $8.4 \pm 2.0 \mathrm{~s})$, visited fewer buckets $(2.3 \pm 0.2$ vs. $4.6 \pm$ $0.3)$, had fewer revisits $(0.03 \pm 0.01$ vs. $0.22 \pm 0.06)$, and tended to have a shorter latency to reach the baited bucket $(55.3 \pm 5.5$ vs. $71.2 \pm 8.4 \mathrm{~s})$ than during the individual visits in phase 1 (Table 5.3). Moreover, the latency to visit the first bucket and the number of revisits were affected by an interaction between pre-weaning housing treatment and batch. Non-informed MS pigs in batch 2 took the most time to visit the first bucket, compared with noninformed FC pigs in batch 2 and both non-informed MS and FC pigs in batch 1. Non-informed FC pigs in batch 1 had more revisits (numerically in the individual visit) than non-informed MS pigs in batch 1 and FC pigs in batch 2 .

\section{Interactions between I and NI pigs}

There was no difference between the MS and FC pigs in the proportions of INI visits (relative to the total number of bucket visits of the NI pig), closely followed trials (which occurred in all pens), and trials in which displacements from NI pigs towards I pigs occurred (Table 5.3). The proportion of trials with displacements was, however, lower in batch 1 than in batch 2. Within MS pairs, there were no trials in which NI pigs showed displacement in batch 1 . This was significantly different from the proportion of trials with displacements within FC pairs of batch 1 (14 out of $28, P<0.0001$ ) and MS pairs of batch 2 (21 out of $28, P<0.0001$ ). Furthermore, in batch 2 , the proportion of trials with displacements was higher within MS pairs than within FC pairs (21 out of 28 vs. 12 out of $28, P=0.03$ ). I pigs rarely displaced NI pigs; the proportion of trials in which an I pig displaced an NI pig was $0.02 \pm 0.01$. The proportion of trials in which I pigs ate from the bait tended to be higher than the proportion of trials in which NI pigs ate from the bait $(0.79 \pm 0.04$ vs. $0.47 \pm 0.07, P<$ $0.10)$.

Overall, during the first 28 trials of phase 2, the percentage of trials in which the I pig ate from the baited bucket was positively correlated with the NI pigs' mean latency to visit the first bucket $(\mathrm{r}=0.62, P=0.01$, Figure 5.4A). More specifically, this correlation was significant for MS pigs ( $\mathrm{r}=0.79, P=0.02)$, but not for FC pigs $(\mathrm{r}=0.46, P=0.26)$. Moreover, the percentage of trials in which the I pig ate from the baited bucket was negatively correlated with the mean 

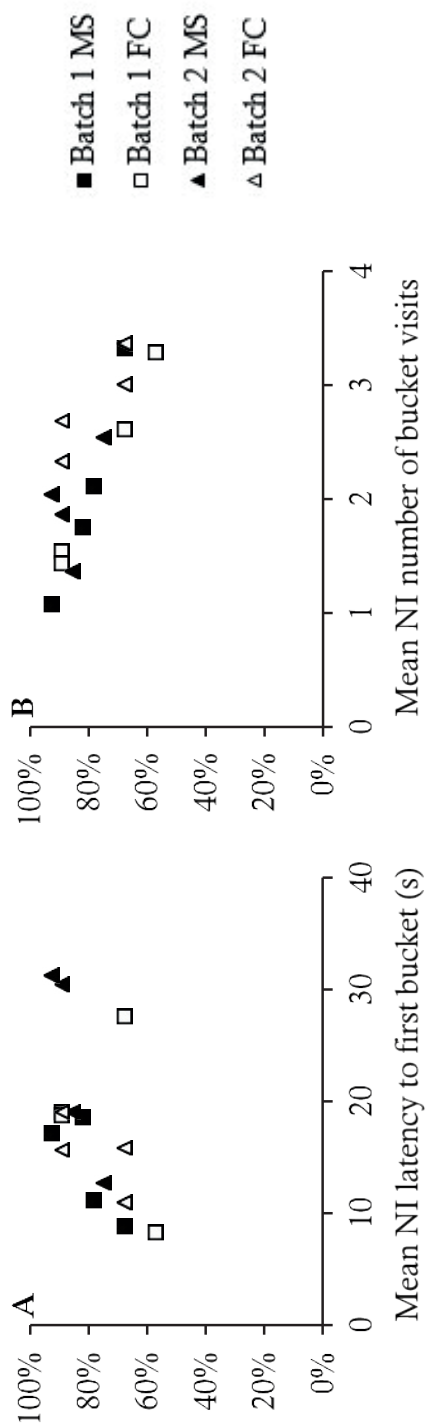

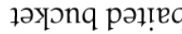

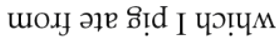

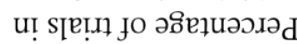

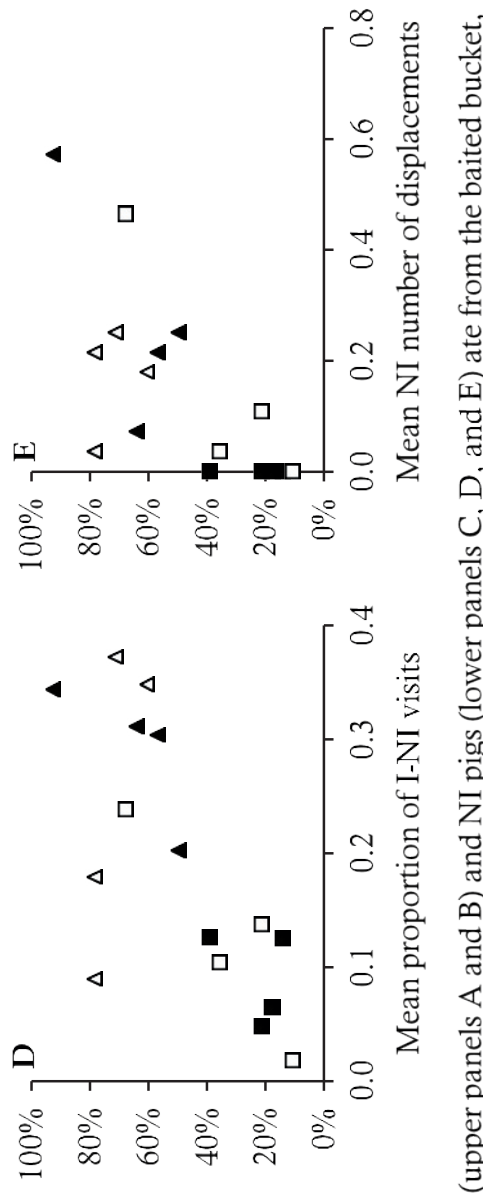

5 
number of bucket visits per trial by the NI pigs ( $\mathrm{r}=-0.73, P=0.001$, Figure 5.4B). This correlation was significant for both MS and FC pigs. Regarding the NI pigs, the percentage of trials in which they ate from the baited bucket was overall positively correlated with i) the mean number of bucket visits per trial by the NI pig ( $\mathrm{r}=0.62, P=0.01$, Figure $5.4 \mathrm{C})$, ii) the mean proportion of I-NI visits per trial ( $\mathrm{r}=0.66, P=0.01$, Figure $5.4 \mathrm{D})$, and iii) the mean number of displacements by the NI pig per trial $(\mathrm{r}=0.74, P=0.001$, Figure $5.4 \mathrm{E})$. The first correlation was, however, significant for the FC pigs $(\mathrm{r}=0.77, P=0.03)$ but not for the MS pigs ( $\mathrm{r}=0.36, P=0.39$ ). The latter 2 correlations were significant for the MS pigs ( $\mathrm{r}=0.90, P=0.002$ and $\mathrm{r}=0.84, P=0.01$, respectively) but not for the FC pigs ( $\mathrm{r}=0.35, P=0.40$ and $\mathrm{r}=0.49, P=0.22$, respectively).

\subsection{Discussion}

It was hypothesised that pigs reared in an MS system would be better prepared to deal with social and cognitive challenges later in life, because they have experienced more complexity in their physical and social environment than conventionally reared pigs.

\subsubsection{FCT}

Based on the study of De Jonge et al. (1996), it was expected that MS pigs would show less aggression and establish a clearer dominance hierarchy than FC pigs during the FCT, characterised by e.g. less retaliation of the submissive pig and more feed access of the dominant pig. There were, however, no differences between MS and FC pigs in the total frequency of aggression and in the absolute difference in aggression within pairs. If anything, FC pigs showed some indications of establishing a clearer hierarchy. For instance, FC pigs showed more asymmetry in the number of bucket access bouts within pairs than MS pigs. This may indicate that the dominant pigs defended the bucket better against the submissive pigs in FC pairs than in MS pairs, i.e. after the dominant pig lifted its head from the bucket in between eating bouts, it prevented the other pig from accessing the feed. Moreover, when considering only the selected dominant-submissive pairs used in the IFT, dominant FC pigs performed head knocks, bites, and pushes twice as often as submissive FC pigs, whereas dominant and submissive MS pigs showed no difference in the mean frequency of these behaviours. Possibly, the tendency of FC pairs to establish a 
clearer hierarchy than MS pairs is related to a difference in the context of obtaining feed in the MS and FC systems; before weaning, MS pigs were stimulated to eat together in the communal feeding area, whereas FC pigs had only 1 relatively small piglet feeder for the whole litter and therefore had a more competitive environment to obtain solid feed. Furthermore, the discrepancies in results with the study of De Jonge et al. (1996) may be explained by the larger contrast in pre-weaning conditions in their study (tethered sows in the control group and larger groups of 8 sows and their piglets in the MS system, with outdoor access) combined with a longer exposure time (weaning at 6 weeks of age in their study, compared with 4 weeks of age in our study). The period of most rapid brain growth occurs until about 6 weeks of age (Dickerson and Dobbing, 1967), which may be the most sensitive period for environmental conditions to affect aspects of social and cognitive development. As the MS and FC pigs in our study were already housed under the same conditions between 4 to 6 weeks of age, the postweaning environment and/or the difference in transition from pre-weaning to post-weaning housing for MS and FC pigs, may have partly overruled effects of pre-weaning housing conditions.

Lastly, anecdotally, we noticed behavioural differences which may be related to problem-solving; FC pigs more often walked away from the bucket after not being able to gain access to the feed ( $7 / 32$ pairs) and subsequently turned to the experimenters ( $3 / 32$ pairs), e.g. looking at them or standing up against the pen partition with their front legs (observations were obtained from remarks noted during testing). These behaviours were not noted for MS pairs. Directing attention towards humans in a challenging situation has also been described in other species, e.g. for goats in an 'unsolvable problem' task (Nawroth et al., 2016).

\subsubsection{IFT phase 1}

It was hypothesised that, in phase 1 of the IFT, informed MS pigs would learn their task faster and would demonstrate a better working memory than informed FC pigs. Overall, the I pigs learned their task, indicated by the reduction in bucket visits, revisits, and latency to reach the bait between search and relocation visits. The performance of the I pigs improved over time, as the latency to reach the baited bucket decreased over the first 9 testing days. These 
variables were, however, not affected by the pre-weaning housing system and MS and FC pigs needed a similar number of trials to reach the criterion to proceed to phase 2 of the IFT, had a similar number of revisits to buckets (reflecting working memory, see van der Staay et al. 2012), and a similar percentage of days with a perseverance error. This is in contrast with studies in which pigs housed under enriched conditions demonstrated a better short term and/or long-term memory than pigs housed under barren conditions (de Jong et al., 2000; Bolhuis et al., 2013; Grimberg-Henrici et al., 2016). In these studies, pigs were, however, housed in contrasting environments at the time of the cognitive tests, whereas in our study pigs were housed under the same conditions at the time of testing and had been in those conditions for the 4 weeks preceding the start of phase 1 of the IFT. Similar to the results of the FCT, the timing and duration of exposure to the contrasting environments may have limited the potential effects of pre-weaning housing conditions on the aspects of cognitive performance tested here (also see Bolhuis et al., 2006; Munsterhjelm et al., 2009).

Interestingly, there were some effects of pre-weaning housing treatment on the performance of the NI pigs during their search visits. The non-informed MS pigs took more time to complete the test, as their latency to reach the bait was 1.4 times longer, while tending to visit fewer buckets than the non-informed FC pigs. When foraging, pigs are able to use both a win-stay strategy, where a previously baited location should be revisited (Mendl et al., 1997), and a winshift strategy, where a previously baited location should be avoided (Laughlin and Mend1, 2000). A win-shift strategy may have been more frequently reinforced in MS pigs, as the pre-weaning MS system provided multiple locations where feed, foraging materials, and even milk could be obtained, whereas a win-stay strategy would have been more strongly reinforced in the FC system in which resources were available in one fixed location only. Possibly, non-informed MS pigs performed more efficiently, albeit slower, because they had to use only the familiar win-shift strategy. On the other hand, pre-weaning housing treatment effects were not present in the search visits of the I pigs, possibly because their cognitive abilities were challenged more by the more complex and varied task that they faced, involving the use of a winstay strategy within a trial and a win-shift strategy between trials. Switching between strategies can reduce pigs' performance, as this seems to be more difficult than using only one strategy (Laughlin and Mendl, 2000). Lastly, the 
longer latency to reach the bait for the non-informed MS pigs compared with the non-informed FC pigs, might be related to a potentially lower urgency of responding in a food-related situation, due to the less competitive pre-weaning environment to obtain solid feed.

In short, informed MS and FC pigs learned their task to remember the specific location of a baited bucket after a search visit and a subsequent relocation visit equally well. Non-informed MS pigs, however, searched differently than noninformed FC pigs, with a longer latency to reach the bait, while visiting fewer buckets.

\subsubsection{IFT phase 2}

During the paired visits in phase 2, I and NI pigs both took more time to reach the first bucket, visited fewer buckets, and had fewer revisits than during the last 8 trials with individual visits in phase 1 . This indicates that both I and NI pigs were more hesitant to start visiting buckets in the paired trials, but thereafter searched more efficiently for the bait. The latency to reach the baited bucket did not differ between phase 1 and phase 2 for the I pigs, but the NI pigs tended to reach the baited bucket quicker when they were tested together with the I pig than when the NI pigs searched for the bait alone. This may suggest that the NI pigs benefited from visiting the testing arena together with the I pig, but that this did not necessarily disadvantage the I pig (also, the proportion of trials in which a pig ate from the baited bucket tended to be higher for I pigs than for NI pigs during paired visits). Potentially disadvantageous effects of the presence of the NI pig during paired visits for the success of the I pig, may depend on the NI pig's own investment in searching for the bait, as suggested by the negative correlation between the percentage of trials in which the I pig ate from the baited bucket and the number of bucket visits by the NI pigs.

Surprisingly, there were relatively few trials in which the NI pig followed the I pig and subsequently displaced the I pig from the baited bucket. Overall, we found lower proportions of I-NI visits $(0.19$ vs. 0.28$)$ and trials with displacements (0.42 vs. 0.65$)$ than reported in the study of Held et al. (2000). This discrepancy may have several explanations. Possibly, the dominance relationship within the I-NI pairs was less clear in our study, or changed over time (also see Meese and Ewbank, 1972, 1973). To check the latter, the FCT was repeated at the end of batch 2 (day 79, with $400 \mathrm{~g}$ of feed per bucket) for 
the 8 I-NI pairs only. All NI pigs were still clearly dominant over the I pigs, with exception of $1 \mathrm{MS}$ pair and $1 \mathrm{FC}$ pair in which dominance was less obvious, thus indicating some stability in the dominance relationships. Furthermore, Held et al. (2000) used a different feed deprivation method, i.e. restricting daily feed provision to $70 \%$ of ad libitum intake, and a different type and quantity of bait, i.e. $400 \mathrm{~g}$ of feed. Possibly, this resulted in a higher motivation to perform the IFT than in our study. Also, Held et al. (2000) ran more trials (48 to 72 ) in phase 2 , but in our study, the proportion of I-NI visits and number of trials with displacements did not increase over time (data not shown), hence running extra trials may not have resulted in more close following of the I pig by the NI pig. Lastly, pigs were tested at a younger age in our study. It has been reported, however, that wild boar piglets and older yearlings have a similar probability to have a certain forager role (Focardi et al., 2015), so the age difference likely did not affect the ability of the pigs to adopt a scrounger role and display close following and displacement as part of their foraging strategy.

The differences in performance between both batches may provide some further insight into factors that may influence the interactions between the I and NI pig. In batch 2 , the proportion of trials with displacements was higher than in batch 1 (0.59 vs. 0.25$)$. Moreover, the proportions of I-NI visits $(0.27 \pm$ 0.04 vs. $0.11 \pm 0.02)$ and closely followed trials $(0.30 \pm 0.05$ vs. $0.09 \pm 0.02)$ were numerically higher in batch 2 than in batch 1 . This may indicate that the changes in bait and testing procedure affected the pigs' performance, although batch effects unrelated to the testing protocol may also have played a role. Interestingly, MS pigs had more trials with displacements in batch 2 than in batch 1, whereas there was no batch effect for FC pigs. In addition, noninformed MS pigs had a longer latency to visit the first bucket in batch 2, compared with FC pigs in batch 2 and both MS and FC pigs in batch 1. This may suggest that non-informed MS pigs in batch 2 awaited the behaviour of the informed pig, rather than directly searching for the bait itself. It could be speculated that MS pigs may not have understood their task in batch 1 and needed the extra testing time given in batch 2. Alternatively, non-informed MS pigs may have adopted a scrounger role -where the submissive pig is exploitedonly if more profitable bait could be obtained. The social organisation of wild boar is characterised by cooperation, rather than competition (Focardi et al., 
2015), and domestic pigs can alter their foraging behaviour in response to the quantity of bait that can be retrieved (Held et al., 2005).

Further indications that MS and FC pigs may differ in their response to the paired visits are derived from the significant correlations of several behaviours with the percentage of trials in which the I and NI pig ate from the baited bucket (although, the correlations should be interpreted with caution given the limited number of pens per batch and treatment). Among MS pairs, the I pig seemed to be more successful (i.e. able to eat from the bait) if the NI pig was slow to visit the first bucket, and the NI pig seemed to be more successful if it followed and displaced the I pig. For FC pigs, however, NI pigs seemed to be more successful if they visited many buckets, which suggests that their success depended more on their own investment in searching for the bait, compared with the MS pigs. The success of an MS pig seemed to depend more on the behaviour of its partner. It can be speculated that, during the paired visits, noninformed FC pigs stayed with the producer role that was imposed during the individual visits, whereas MS pigs were more flexible in changing their foraging strategy (i.e. towards NI pigs adopting a scrounger role and I pigs adjusting their behaviour to limit exploitation). Underlying may be a different predisposition for a certain coping style during rearing in either the MS or FC system, as characteristics such as behavioural flexibility and use of social information are usually attributed to a reactive coping style, whereas more rigid behaviour is characteristic of a pro-active coping style (Benus et al., 1990; Koolhaas et al., 1999; Coppens et al., 2010; Kurvers et al., 2010a; Kurvers et al., 2010b).

Thus, both I and NI pigs changed their behaviour in response to being tested pairwise instead of individually. Overall, effects of pre-weaning housing treatment were not distinctly present and partly depended on batch-related differences. There were, however, indications that MS pigs made more use of social information than FC pigs during the paired visits.

\subsection{Conclusions}

To conclude, pre-weaning housing in either a complex multi-suckling system or a conventional farrowing system had few distinct effects on post-weaning social and cognitive performance. There were, however, some indications that FC pigs established clearer dominance relationships than MS pigs in a feed 
competition test. During individual training in an informed forager test, informed MS and FC pigs performed similarly in learning their task. During the paired visits of the informed forager test, however, there were indications that MS and FC pigs adopted different foraging strategies, as non-informed MS pigs seemed to make more use of the information of their informed partner.

\subsection{Acknowledgements}

The authors gratefully acknowledge the Ministry of Economic Affairs for their financial support and the staff of Swine Innovation Centre Sterksel and Research Facility Carus for their assistance and care for the animals. Furthermore, the authors want to thank Fleur Bartels, Monique Ooms, Bjorge Laurenssen, Nick Timmermans, and Hilde de Kok for their assistance during various parts of this experiment. Lastly, Caroline Clouard is acknowledged for providing valuable feedback on the manuscript. 


\subsection{References}

Arbilly M., Weissman D.B., Feldman M.W., and Grodzinski U. 2014. An arms race between producers and scroungers can drive the evolution of social cognition. Behavioral Ecology 25: 487-495.

Beauchamp G. and Giraldeau L.-A. 1997. Patch exploitation in a producer-scrounger system: test of a hypothesis using flocks of spice finches (Lonchura punctulata). Behavioral Ecology 8: 54-59.

Benus R., Den Daas S., Koolhaas J., and Van Oortmerssen G. 1990. Routine formation and flexibility in social and non-social behaviour of aggressive and non-aggressive male mice. Behaviour 112: 176-193.

Bolhuis J.E., Oostindjer M., Hoeks C.W.F., de Haas E.N., Bartels A.C., Ooms M., and Kemp B. 2013. Working and reference memory of pigs (Sus scrofa domesticus) in a holeboard spatial discrimination task: the influence of environmental enrichment. Animal Cognition 16: 845-850.

Bolhuis J.E., Schouten W.G.P., Schrama J.W., and Wiegant V.M. 2006. Effects of rearing and housing environment on behaviour and performance of pigs with different coping characteristics. Applied Animal Behaviour Science 101: 6885.

Coppens C.M., de Boer S.F., and Koolhaas J.M. 2010. Coping styles and behavioural flexibility: towards underlying mechanisms. Philosophical Transactions of the Royal Society of London B: Biological Sciences 365: 4021-4028.

Croney C.C. and Newberry R.C. 2007. Group size and cognitive processes. Applied Animal Behaviour Science 103: 215-228.

D'Eath R.B. 2005. Socialising piglets before weaning improves social hierarchy formation when pigs are mixed post-weaning. Applied Animal Behaviour Science 93: 199-211.

de Jong I.C., Prelle I.T., van de Burgwal J.A., Lambooij E., Korte S.M., Blokhuis H.J., and Koolhaas J.M. 2000. Effects of environmental enrichment on behavioral responses to novelty, learning, and memory, and the circadian rhythm in cortisol in growing pigs. Physiology \& behavior 68: 571-578. 
De Jonge F.H., Bokkers E.A.M., Schouten W.G.P., and Helmond F.A. 1996. Rearing piglets in a poor environment: Developmental aspects of social stress in pigs. Physiology and Behavior 60: 389-396.

Dickerson J. and Dobbing J. 1967. Prenatal and postnatal growth and development of the central nervous system of the pig. Proceedings of the Royal Society of London B: Biological Sciences 166: 384-395.

Focardi S., Morimando F., Capriotti S., Ahmed A., and Genov P. 2015. Cooperation improves the access of wild boars (Sus scrofa) to food sources. Behavioural processes 121: 80-86.

Giraldeau L.-A. and Lefebvre L. 1986. Exchangeable producer and scrounger roles in a captive flock of feral pigeons: a case for the skill pool effect. Animal Behaviour 34: 797-803.

Godfrey-Smith P. 2002. Environmental complexity and the evolution of cognition. The evolution of intelligence: 233-249.

Grimberg-Henrici C.G.E., Vermaak P., Elizabeth Bolhuis J., Nordquist R.E., and van der Staay F.J. 2016. Effects of environmental enrichment on cognitive performance of pigs in a spatial holeboard discrimination task. Animal Cognition 19: 271-283.

Gundlach H. 1968. Brutfürsorge, Brutpflege, Verhaltensontogenese und Tagesperiodik beim Europäischen Wildschwein (Sus scrofa L.)1. Zeitschrift für Tierpsychologie 25: 955-995.

Held S., Baumgartner J., Kilbride A., Byrne R.W., and Mendl M. 2005. Foraging behaviour in domestic pigs (Sus scrofa): Remembering and prioritizing food sites of different value. Animal Cognition 8: 114-121.

Held S., Mendl M., Devereux C., and Byrne R.W. 2000. Social tactics of pigs in a competitive foraging task: The 'informed forager' paradigm. Animal Behaviour 59: 569-576.

Held S., Mendl M., Devereux C., and Byrne R.W. 2002. Foraging pigs alter their behaviour in response to exploitation. Animal Behaviour 64: 157-165.

Held S.D.E., Byrne R.W., Jones S., Murphy E., Friel M., and Mendl M.T. 2010. Domestic pigs, Sus scrofa, adjust their foraging behaviour to whom they are foraging with. Animal Behaviour 79: 857-862. 
Hessel E.F., Reiners K., and Van Den Weghe H.F.A. 2006. Socializing piglets before weaning: Effects on behavior of lactating sows, pre- and postweaning behavior, and performance of piglets. Journal of Animal Science 84: 28472855.

Jansen J., Bolhuis J.E., Schouten W.G., Spruijt B.M., and Wiegant V.M. 2009. Spatial learning in pigs: effects of environmental enrichment and individual characteristics on behaviour and performance. Animal cognition 12: 303-315.

Jensen P. 1986. Observations on the maternal behaviour of free-ranging domestic pigs. Applied Animal Behaviour Science 16: 131-142.

Kanaan V.T., Lay Jr D.C., Richert B.T., and Pajor E.A. 2012. Increasing the Frequency of Co-Mingling Piglets During the Lactation Period Alters the Development of Social Behavior Before and After Weaning. Journal of Applied Animal Welfare Science 15: 163-180.

Koolhaas J.M., Korte S.M., De Boer S.F., Van Der Vegt B.J., Van Reenen C.G., Hopster H., De Jong I.C., Ruis M.A.W., and Blokhuis H.J. 1999. Coping styles in animals: current status in behavior and stress-physiology. Neuroscience \& Biobehavioral Reviews 23: 925-935.

Kurvers R.H.J.M., Prins H.H.T., Van Wieren S.E., Van Oers K., Nolet B.A., and Ydenberg R.C. 2010a. The effect of personality on social foraging: Shy barnacle geese scrounge more. Proceedings of the Royal Society B: Biological Sciences 277: 601-608.

Kurvers R.H.J.M., van Oers K., Nolet B.A., Jonker R.M., van Wieren S.E., Prins H.H.T., and Ydenberg R.C. 2010b. Personality predicts the use of social information. Ecology Letters 13: 829-837.

Laughlin K. and Mendl M. 2000. Pigs shift too: foraging strategies and spatial memory in the domestic pig. Animal Behaviour 60: 403-410.

Li Y. and Wang L. 2011. Effects of previous housing system on agonistic behaviors of growing pigs at mixing. Applied Animal Behaviour Science 132: 20-26.

McGlone J.J. and Curtis S.E. 1985. Behavior and performance of weanling pigs in pens equipped with hide areas. Journal of Animal Science 60: 20-24. 
Meese G. and Ewbank R. 1972. A note on instability of the dominance hierarchy and variations in level of aggression within groups of fattening pigs. Animal Production 14: 359-362.

Meese G. and Ewbank R. 1973. The establishment and nature of the dominance hierarchy in the domesticated pig. Animal Behaviour 21: 326-334.

Mendl M., Laughlin K., and Hitchcock D. 1997. Pigs in space: spatial memory and its susceptibility to interference. Animal Behaviour 54: 1491-1508.

Meynhardt H. 1980. Schwarzwild-Report: Vier Jahre unter Wildschweinen. Verlag J. Neumann-Neudamm, Berlin, Germany.

Munsterhjelm C., Peltoniemi O.A.T., Heinonen M., Hälli O., Karhapää M., and Valros A. 2009. Experience of moderate bedding affects behaviour of growing pigs. Applied Animal Behaviour Science 118: 42-53.

Nawroth C., Brett J.M., and McElligott A.G. 2016. Goats display audience-dependent human-directed gazing behaviour in a problem-solving task. Biology Letters 12.

Oostindjer M., van den Brand H., Kemp B., and Bolhuis J.E. 2011. Effects of environmental enrichment and loose housing of lactating sows on piglet behaviour before and after weaning. Applied Animal Behaviour Science 134: 31-41.

Petersen V., Recén B., and Vestergaard K. 1990. Behaviour of sows and piglets during farrowing under free-range conditions. Applied animal behaviour science 26: 169-179.

Samarakone T.S. and Gonyou H.W. 2009. Domestic pigs alter their social strategy in response to social group size. Applied Animal Behaviour Science 121: 8-15.

Sneddon I., Beattie V., Dunne L., and Neil W. 2000. The effect of environmental enrichment on learning in pigs. Animal Welfare 9: 373-383.

Spinka M., Newberry R.C., and Bekoff M. 2001. Mammalian play: training for the unexpected. Quarterly Review of Biology: 141-168.

Stolba A. and Wood-Gush D. 1989. The behaviour of pigs in a semi-natural environment. Animal production 48: 419-425.

van der Staay F.J., Gieling E.T., Pinzón N.E., Nordquist R.E., and Ohl F. 2012. The appetitively motivated " cognitive" holeboard: A family of complex spatial 
discrimination tasks for assessing learning and memory. Neuroscience and Biobehavioral Reviews 36: 379-403.

van Nieuwamerongen S.E., Soede N.M., van der Peet-Schwering C.M.C., Kemp B., and Bolhuis J.E. 2015. Development of piglets raised in a new multi-litter housing system vs. conventional single-litter housing until 9 weeks of age. Journal of Animal Science 93: 5442-5454.

Verdon M., Morrison R.S., and Hemsworth P.H. 2016. Rearing piglets in multi-litter group lactation systems: Effects on piglet aggression and injuries postweaning. Applied Animal Behaviour Science.

Weng R.C., Edwards S.A., and English P.R. 1998. Behaviour, social interactions and lesion scores of group-housed sows in relation to floor space allowance. Applied Animal Behaviour Science 59: 307-316. 



\section{CHAPTER 6}

\section{Gradual weaning improves performance and behaviour of pigs raised in a multi-suckling system}

S.E. van Nieuwamerongen ${ }^{1 *}$, N.M. Soede ${ }^{1}$, C.M.C. van der PeetSchwering $^{2}$, B. Kemp ${ }^{1}$, J.E. Bolhuis ${ }^{1}$

${ }^{1}$ Adaptation Physiology Group, Department of Animal Sciences, Wageningen University \& Research, PO Box 338, 6700 AH Wageningen, The Netherlands; ${ }^{2}$ Wageningen Livestock Research, PO Box 338, 6700 AH Wageningen, The Netherlands.

*Corresponding author: S.E. van Nieuwamerongen at Sofie.vannieuwamerongen@wur.nl.

Under revision at Applied Animal Behaviour Science (2017) 


\subsection{Abstract}

We studied effects of 2 weaning procedures on the development of pigs raised in a multi-suckling (MS) system with 5 sows and their litters. One MS group was subjected to gradual weaning during a lactation period of 9 weeks, which was stimulated by forced intermittent-suckling (IS) for 10 hours per day during the fifth week of lactation, followed by a 4 -week period in which sows could voluntarily get away from their piglets (IS9 treatment). The other MS group was weaned abruptly at 4 weeks of age and was subsequently housed in a nursery (A4 treatment). At 9 weeks of age, pigs from both treatments were relocated to a finishing unit, where they were housed in a group of maximum 35 pigs per treatment. Five consecutive batches of 10 sows and their litters were studied. Weaning had a more profound impact for the A4 pigs than IS and sow-initiated separation did for the IS9 pigs, demonstrated by a lower weight gain between days 27 and $33(0.90 \pm 0.08$ vs. $1.51 \pm 0.06 \mathrm{~kg} / \mathrm{pig}, P<0.01)$, indications of a higher diarrhoea occurrence, a distinct peak in belly-nosing behaviour, and numerically higher levels of aggression and damaging oral manipulation between weeks 4 to 9 . For IS9 pigs, weaning seemed to have less impact; feed intake after transition to the finishing unit was similar in both treatments, indicating that IS9 pigs had a more successful transition to a diet of only solid feed, and IS9 pigs showed no growth check, nor behavioural indicators of having difficulty coping with the post-weaning situation. The gradual and extended weaning process likely gradually prepared the gastrointestinal tract to process solid feed, which may explain the better performance of the IS9 pigs. Additionally, benefits of the gradual weaning treatment were reflected in behavioural differences over the entire experiment; between 4 to 18 weeks of age, IS9 pigs overall showed less belly-nosing, less damaging oral manipulation, and had fewer lesions related to manipulation and aggression than A4 pigs. This may altogether indicate a less stressed state of the IS9 pigs. To conclude, IS9 pigs coped better with both transitions than A4 pigs did and the gradual weaning treatment had long-term beneficial effects, particularly concerning behaviour. Therefore, gradual weaning in a multi-suckling system seems promising for improving piglet performance, behaviour, and welfare.

Key words: multi-suckling system, get-away system, intermittent-suckling, gradual weaning, behaviour, pigs 


\subsection{Introduction}

Recently, group housing of sows has become mandatory during the major part of gestation in the EU. During lactation, however, individual confinement of sows is still common. This type of housing is being criticised due to welfare concerns for the sows (Baxter et al., 2012) and because it provides a suboptimal environment for piglet development. Previously, we studied an alternative system that better meets the natural behavioural needs of sows and piglets around farrowing and lactation (van Nieuwamerongen et al., 2015). The system consisted of multi-suckling (MS) housing for 5 sows and their litters, including loose farrowing pens and a communal area. For the sows, MS housing better enables behavioural expression and provides a more gradual transition between group housing during gestation and lactation. Piglets raised in the MS system (housed post-weaning in an enriched pen with 40 piglets) showed improved pre- and post-weaning performance compared with piglets raised conventionally with a crated sow (housed post-weaning in groups of 10 litter-mates in a standard pen). MS piglets showed more feed-directed behaviour and less damaging oral manipulation, like tail biting, before weaning than piglets in the conventional system. In the (early) post-weaning phase, piglets raised in the MS system showed a higher feed intake, a higher weight gain, a lower occurrence of diarrhoea, more play behaviour, and less damaging oral manipulation than conventionally reared piglets. In the previous study, piglets were, however, weaned abruptly at 4 weeks of age. To further improve piglet development, weaning at a later age combined with a more gradual weaning process may be beneficial. For instance, forced intermittent-suckling (IS) -during which sows were separated from their piglets for 10 to 12 hours per day- combined with an extended lactation of 33 to 45 days, resulted in a higher feed intake, a reduced growth check, and improved gut characteristics post-weaning, compared with piglets weaned conventionally at 3 to 4 weeks of age (Berkeveld et al., 2007b; Berkeveld et al., 2009). Additionally, in a system in which sows could voluntarily leave their piglets during a lactation period of 5 weeks, piglets had a $27 \%$ higher weight gain and a $31 \%$ higher feed intake in the first 2 weeks post-weaning than piglets that were kept together with the sow during lactation (Pajor et al., 1999). As gradual weaning may benefit development of pigs raised in an MS system, the aim of the current study was to investigate short- and long-term effects of 2 different weaning procedures; MS pigs were either weaned abruptly at 4 weeks of age, or weaned more 
gradually during a 9-week lactation period, which included forced IS in the fifth week of lactation, after which sows could voluntarily separate themselves from the piglets.

\subsection{Materials and methods}

\subsubsection{Animals and housing}

The experiment was approved by the Animal Care and Use Committee of Wageningen University \& Research (AVD10400201551). Five consecutive batches of 10 multiparous Topigs 20 sows and their litters (Tempo x Topigs 20) were studied at the animal facilities of Swine Innovation Centre Sterksel, the Netherlands. Per batch, 2 groups of 5 sows and their litters were housed in 2 separate adjacent multi-suckling units (Figure 6.1). Within each batch, groups

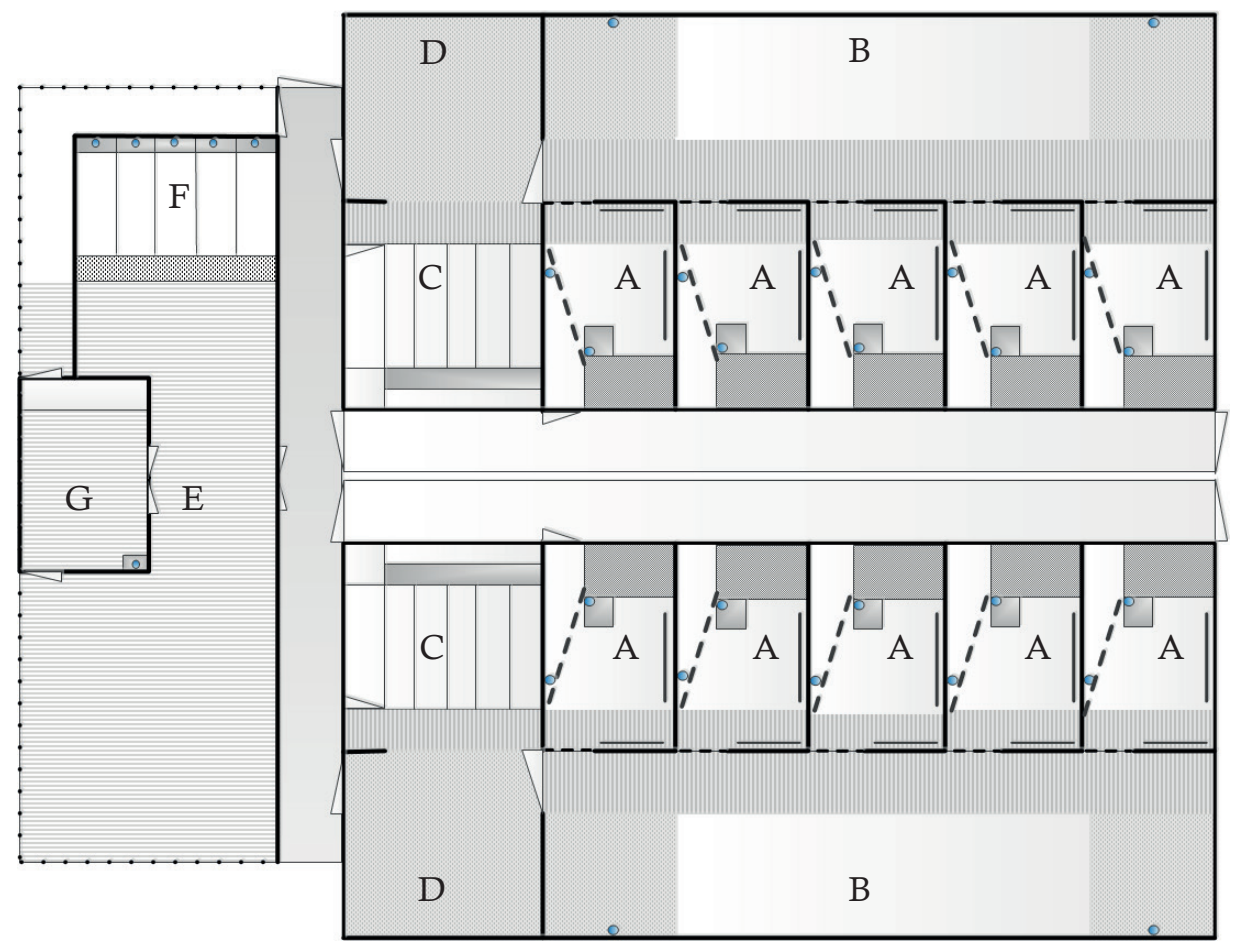

Figure 6.1. Layout of the system with 2 separate indoor multi-suckling units connected to a covered outdoor intermittent-suckling area. Each multi-suckling unit had 5 farrowing pens (A: with piglet nest, sow feeder, drinkers, and anti-crushing devices) connected to a communal area with a lying area (B: with 2 drinkers), feeding area (C: with 5 sow feeding places surrounded by an area accessible to only the piglets, containing piglet feeders), and a dunging area (D). The intermittent-suckling area (E) contained feeding stalls (F) and a boar pen (G). 
of sows and their piglets were subjected to one of 2 treatments differing in weaning procedure (Figure 6.2). The treatments, termed IS9 and A4, were allocated per multi-suckling unit. IS9 piglets were subjected to forced IS from 4 to 5 weeks of age, during which sows were separated from the piglets for 10 hours per day. The period of IS was followed by a 4-week period during which sows could voluntarily get away from the piglets until weaning at 9 weeks of age. A4 piglets were weaned abruptly at 4 weeks of age and were subsequently housed in a nursery unit until 9 weeks of age. Pigs from both treatments were relocated to the same finishing unit at 9 weeks of age where they were housed as one group per treatment until the end of the experiment.
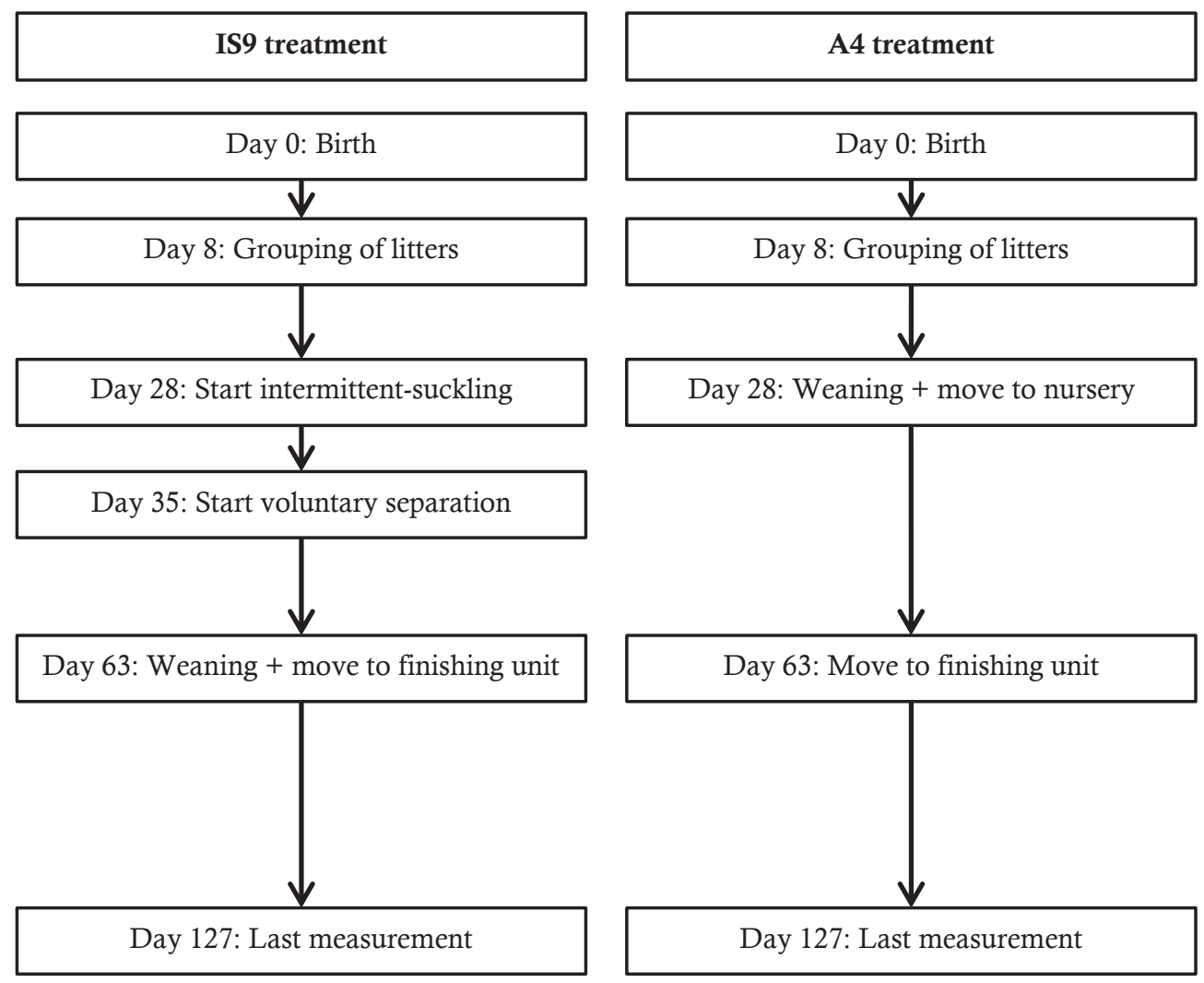

Figure 6.2. Schematic overview of the experimental design. The 2 treatment groups, termed IS 9 and A4, differed in weaning procedure. IS9 piglets were subjected to intermittent-suckling from 4 to 5 weeks of age, during which sows were separated from the piglets for 10 hours per day. Thereafter, sows could voluntarily get away from the piglets until weaning on day 63. A4 piglets were weaned abruptly at 4 weeks of age and were subsequently housed in a nursery unit until 9 weeks of age. Pigs from both treatments were relocated to a finishing unit at 9 weeks of age, where they were housed under equal conditions until the end of the experiment. 


\section{Weeks 0 to 4}

One week before expected farrowing, sows were transferred to one of the 2 multi-suckling units. Sows had the same expected farrowing date and distribution across the 2 units was balanced for parity (on average $3.4 \pm 0.2$ ). Per unit, sows originated from the same stable gestation group. Each unit had 5 farrowing pens connected to a communal area (Figure 6.1). The farrowing pens were $3.2 \times 2.2 \mathrm{~m}$ each and contained a feed trough for the sow, a water nipple for the piglets, and a covered piglet nest of $0.7 \times 1.6 \mathrm{~m}$ with heated solid flooring. The floor in the rest of the pen consisted of solid concrete and concrete slats. Five hessian sacks were provided per farrowing pen as nesting material. Sows could access all farrowing pens and communal areas from the moment of entry, with the exception of the nights before farrowing; each sow was locked in her own farrowing pen daily between 16:30 and 07:30 $\mathrm{h}$ from the moment of entry, until farrowing had ended. Piglets were ear tagged and weighed within 24 hours post-partum (p.p.). Litter sizes were standardised between 24 and 48 hours p.p. according to the number of functional teats available per sow, with a maximum of 14 piglets per litter. From day 2 p.p., 2 handfuls of long-stemmed straw were provided daily per farrowing pen. Piglets were tail docked and received an iron injection within 4 days p.p.

The communal area was divided in an area for feeding $(3.2 \times 3.3 \mathrm{~m}$, solid concrete and metal slats), defecating/urinating ( $2.8 \times 3.3 \mathrm{~m}$, metal slats), and lying ( $2.8 \times 11.0 \mathrm{~m}$, solid concrete and metal slats). As enrichment, 5 hessian sacks and 5 ropes were attached to the partitions surrounding the resting area. Piglets were given access to the whole system at a mean age of $8.1 \pm 0.3$ days. This was done by removing a $31 \mathrm{~cm}$ high piglet barrier at the entrance and a 'piglet door' $(0.4 \times 0.3 \mathrm{~m})$ from each farrowing pen. The communal feeding area contained 5 feeding places for the sows, separated by horizontal metal bars, and a surrounding area with piglet feeders accessible to only the piglets. The sows were floor fed twice daily in the communal area, and piglets could eat together with the sows from the sow feed and from creep feed provided in the piglet feeders. Sows were fed in their own farrowing pen only in the afternoons before farrowing and during the first days after farrowing in case the sows did not leave their pen to eat in the communal area. Otherwise, they were fed in the communal area. 


\section{Weeks 4 to 9}

Based on body weight at 4 weeks of age, on average $34.4 \pm 0.3$ piglets per treatment were selected to be studied further (range 33 to 35 piglets, with an equal number of piglets selected per treatment within a batch). Preferably, 7 piglets were selected from each of the 5 litters within a treatment, with the selected piglets preferably having a body weight closest to the median weight of the complete litter. Between treatments, the groups were balanced for sex ratio $(52.7 \pm 2.0 \%$ males) and for the difference in median body weight of the selected piglets relative to the median body weight of the complete litters $(+0.26 \pm 0.13 \mathrm{~kg})$. The selected IS9 piglets remained among the unselected animals until weaning at 9 weeks of age, i.e. the group of 5 sows and their litters remained intact until relocation of the selected animals to the finishing unit. The selected A4 piglets were relocated to the nursery at 4 weeks of age.

\section{$\underline{\text { IS9 treatment }}$}

From day 28 (i.e. the day of weaning in the A4 group) until day 34, sows and piglets were subjected to forced IS by bringing the sows to an IS area for 10 hours per day (from 06:00 to 16:00 h) during 7 consecutive days. The IS area was located beside the MS units (Figure 6.1). IS was used to stimulate lactational oestrus and ovulation in the sows. During IS, sows had contact with a boar in the IS area. Radios were playing in the IS area and in the MS unit to reduce auditory contact between sows and piglets. From day 35 onward, sows could move freely between the IS area and the MS unit, thus they could voluntarily separate themselves from the piglets. Sows were able to move between these areas by use of a flexible partition that the sows could step over, but the piglets could not. The height of the partition could be adjusted using springs. After the week of forced IS until weaning, no boar was present in the IS area. From day 28 until weaning, sows were fed in the IS area in the morning and in the MS unit in the afternoon. IS9 piglets were weaned at 9 weeks of age.

\section{$\underline{\text { A4 treatment }}$}

At 4 weeks of age, the selected piglets were relocated to one nursery unit measuring $6.2 \times 2.6 \mathrm{~m}$. Flooring consisted of $52 \%$ heated solid concrete and $48 \%$ metal slats. The pen contained 4 feeders, 4 water nipples, 3 chew toys, 4 
hessian sacks, and 8 ropes. Additionally, piglets received long-stemmed straw. The amount increased with age from 4 to 6 handfuls twice daily.

\section{General management of IS9 and A4 animals between weeks 0 to 9}

Sows received a commercially available diet containing 9.5 MJ of NE, 14.9\% $\mathrm{CP}$, and $0.76 \%$ ileal digestible lysine. For both treatments, creep feed (11.6 MJ of $\mathrm{NE}, 17.5 \% \mathrm{CP}$, and $1.17 \%$ ileal digestible lysine) was provided to the piglets in 3 round feeders (diameter $28 \mathrm{~cm}$ ) twice daily from day 12 onward. During days 21 and 22, creep feed was mixed with weaner feed (9.9 MJ of NE, 16.0\% $\mathrm{CP}$, and $0.99 \%$ ileal digestible lysine), after which only weaner feed was provided. During days 43 and 44, weaner feed was mixed with starter feed (9.7 MJ of NE, $15.6 \% \mathrm{CP}$, and $0.94 \%$ ileal digestible lysine), after which only starter feed was provided. For both treatments, feed was provided in 4 feeders with in total 8 feeding places from 4 weeks of age. In addition, the round creep feeders remained in use until 5 weeks of age. Water was available ad libitum. Hessian sacks and ropes were replaced if needed. Animal health was checked twice daily.

\section{The finishing phase}

At 9 weeks of age, selected pigs were relocated to the finishing unit, where they were housed as one group per treatment in adjacent pens (range 31 to 35 pigs, with an equal number of pigs relocated per treatment within a batch). In case A4 pigs died in the nursery before 9 weeks of age, the same number of pigs was deselected in the IS9 group. In case selected IS9 pigs died, became ill, or injured before 9 weeks of age, replacement IS9 pigs were selected the day before relocation. On average $33.2 \pm 0.6$ pigs per treatment were moved to the finishing unit. Groups consisted of $52.7 \pm 2.0 \%$ males and the difference in median body weight of the relocated pigs relative to the complete litters was $+0.28 \pm 0.12 \mathrm{~kg}$. Each pen consisted of an indoor area of $6.0 \times 5.3 \mathrm{~m}$ (with $81 \%$ solid concrete and $19 \%$ metal slats) and a sheltered outdoor area of $6.0 \mathrm{x}$ $2.0 \mathrm{~m}$ (with $75 \%$ concrete slats and $25 \%$ metal slats). Each pen had 4 feeders with one feeding place each, 4 hessian sacks, and 8 ropes attached to the pen partitions indoors, and 4 drinkers were available outside. Hessian sacks and ropes were replaced if needed. Additionally, long-stemmed straw and corn silage were provided twice daily. The total amount of daily provided straw increased with age from 1 to $3 \mathrm{~kg}$ per pen. The total amount of daily provided 
corn silage increased with age from 6 to $18 \mathrm{~kg}$ per pen. Commercially available feed was provided ad libitum. The first grower diet was given from days 63 to 98 and contained $10.0 \mathrm{MJ}$ of $\mathrm{NE}, 16.9 \% \mathrm{CP}$, and $1.03 \%$ ileal digestible lysine. The second grower diet was given from days 98 to 126 and contained $9.7 \mathrm{MJ}$ of NE, $14.8 \% \mathrm{CP}$, and $0.79 \%$ ileal digestible lysine. The finisher diet was given from day 126 until the end of the finishing phase and contained 9.5 MJ of NE, $14.5 \% \mathrm{CP}$, and $0.74 \%$ ileal digestible lysine.

\subsubsection{Measurements}

\section{Observation of nursing behaviour}

The frequency of nursing bouts was observed for the IS9 pigs only, during 24hour periods on days $21,29,35,42,49$, and 56 . Sows and piglets received a mark with stock marker spray on the day before observations to distinguish piglets at litter level. The time, identity of the sow, and the presence or absence of milk let-down were noted for each nursing bout.

\section{Body weight, feed intake, damage scores, and faecal consistency scores}

Pigs were weighed on days $0,27,33,63,68,98$, and 126. Feed intake at group level was determined for days 28 to 33,33 to 63,63 to 68,68 to 98 , and 98 to 126. This included intake of weaner feed, starter feed, and grower feed 1 and 2. Intake of sow feed during days 28 to 33 and days 33 to 63 could not be determined for the IS9 pigs because the intake by the sows and the piglets could not be distinguished. Damage on ears, tail, and skin was scored per pig using the protocols in Table 6.1 on days 62 and 126. Skin scratches were counted as a measure of aggression (Turner et al., 2006), and lesions on ears and tails were scored as a measure of oral manipulation by pen mates. Faecal consistency was scored on days $33,40,68$, and 75 . The anal area of each pig was visually inspected and scored as containing pasty faeces or watery faeces.

Observation of play behaviour, damaging oral manipulation, aggression, bellynosing, and activity

Pigs were marked on the day before observations using stock marker spray to allow individual identification. Frequency of play behaviour, damaging oral manipulation, aggression, and belly-nosing (Table 6.2) was recorded on days 34, 41, 69, 76, and 127 using continuous behaviour sampling. From the 7 
Table 6.1. Damage scoring protocol.

\begin{tabular}{|c|c|}
\hline Score & Description \\
\hline \multicolumn{2}{|c|}{ Ear manipulation damage } \\
\hline 0 & No damage. \\
\hline 1 & Small bite-mark(s), ear is intact. \\
\hline 2 & Small wound(s), ear is intact. \\
\hline 3 & Medium-sized wound(s), ear is intact. \\
\hline 4 & Severe wound(s), part of ear removed. \\
\hline \multicolumn{2}{|c|}{ Tail manipulation damage } \\
\hline 0 & No damage. \\
\hline 1 & Small bite-mark(s), tail is intact. \\
\hline 2 & Small wound(s), tail is intact. \\
\hline 3 & Medium-sized wound(s), part of tail removed. \\
\hline \multicolumn{2}{|r|}{$\underline{\text { Skin lesions }}$} \\
\hline \multicolumn{2}{|r|}{$\begin{array}{l}\text { The number of fresh scratches (with redness) is counted per body region stated below. The length } \\
\text { per scratch is not considered. Superficial and deep lesions are scored separately. }\end{array}$} \\
\hline Front & Backside of ears, neck, shoulders, and front paws (face not included). \\
\hline Middle & Back and flanks. \\
\hline Rear & Hindquarters. \\
\hline
\end{tabular}

selected pigs per litter, 2 males and 2 females were observed on days 34 and 41 . The 4 focal pigs had a body weight closest to the median body weight of the litter present on day 27. On days 69, 76, and 127, all 7 selected pigs per litter were observed. A different number of pigs was observed simultaneously before and after 9 weeks of age ( 4 and 7 per treatment, respectively) because identification of individual pigs was more difficult in the MS system than in the finishing unit. Observations were carried out between 08:00 and 16: $00 \mathrm{~h}$ during 6 time blocks of 1 hour each. Each group of focal pigs (consisting of either 4 or 7 pigs) was observed consecutively for 10 minutes per group and all focal pigs were observed within each time block, resulting in 60 minutes of observations per pig per day. At the start and end of each 10-minute observation of one group of focal pigs, instantaneous scan sampling was used to score their activity (Table 6.2). All observations within and between time blocks were balanced for treatment and observer.

\subsubsection{Statistical analysis}

Data were analysed with SAS 9.2 (SAS Inst. Inc., Cary, NC). Variables were averaged per treatment and batch before analysis, using one group of pigs as one experimental unit (i.e. $N=10$ ). Residuals were checked for normality and 
Table 6.2. Ethogram used for behaviour observations of play, manipulation, aggression, and activity.

\begin{tabular}{|c|c|}
\hline Behaviour & Description \\
\hline \multicolumn{2}{|l|}{ Play behaviour } \\
\hline Individual play & $\begin{array}{l}\text { Scampering (forward hops in rapid succession), turning (rapid turn on the } \\
\text { spot), head tossing, flopping (rapid drop from an upright position to lying), } \\
\text { rolling on back, sliding, or running across pen individually. }\end{array}$ \\
\hline $\begin{array}{l}\text { Social play ( } 2 \text { pigs or } \\
\text { more) }\end{array}$ & $\begin{array}{l}\text { Nudging (play invitation: gentle pushing of opponent), gambolling (running } \\
\text { across pen together), play fighting, or scampering together. }\end{array}$ \\
\hline Substrate play & $\begin{array}{l}\text { Shaking of head while holding material (e.g. straw, rope) that protrudes } \\
\text { from mouth (not scored when only chewing on material). }\end{array}$ \\
\hline \multicolumn{2}{|l|}{ Manipulative behaviour } \\
\hline Manipulating ears & Nibbling, sucking, or chewing an ear of another piglet. \\
\hline Manipulating tails & Nibbling, sucking, or chewing the tail of another piglet. \\
\hline Manipulating sow ${ }^{1}$ & Nibbling, sucking, or chewing part of the body of the sow. \\
\hline Manipulating other & $\begin{array}{l}\text { Nibbling, sucking, or chewing another part of the body of another piglet, } \\
\text { e.g. a paw ('hair sucking/nibbling' not scored). }\end{array}$ \\
\hline Belly-nosing & $\begin{array}{l}\text { Rubbing belly of another pig with up and down snout movements ( } \geq 3 \text { up } \\
\text { and down movements). }\end{array}$ \\
\hline \multicolumn{2}{|l|}{ Aggressive behaviour } \\
\hline Head knocking & $\begin{array}{l}\text { Horizontal or vertical knocking with the head or forward thrusting with the } \\
\text { snout toward another piglet (single event or short series of events). }\end{array}$ \\
\hline Biting & Biting another piglet (single event or short series of events). \\
\hline Fighting & $\begin{array}{l}\text { Intense mutual ramming or pushing (parallel or antiparallel), with or } \\
\text { without biting, in rapid succession. }\end{array}$ \\
\hline Aggression at the feeder & Head knocking or biting, while located at the feeder. \\
\hline \multicolumn{2}{|l|}{ Activity } \\
\hline Inactive lying & $\begin{array}{l}\text { Lying without performing any other behaviour (such as substrate chewing, } \\
\text { sniffing the floor or manipulation). }\end{array}$ \\
\hline Active lying & $\begin{array}{l}\text { Lying while performing other behaviour (such as substrate chewing, } \\
\text { sniffing the floor or manipulation). }\end{array}$ \\
\hline Active & Being in upright position (standing or walking). \\
\hline
\end{tabular}

${ }^{1}$ Pre-weaning only

data were square root transformed if needed. Data of pigs that died were not included in the analyses of feed intake, body weight, and body weight gain. Feed intake at pen level was corrected with the estimated feed consumption prior to a pig's death. Mixed models with weaning treatment and batch as fixed effects were used for the following variables: feed intake, weight gain, 
feed conversion, the number of days piglets had pasty or watery faeces, and damage scores. Body weight, behaviour, and average faecal consistency score (calculated as: no faeces $=0$, pasty faeces $=1$ and watery faeces $=2$ ) were analysed with mixed models including weaning treatment, day of measurement, weaning treatment $\mathrm{x}$ day interaction, and batch. Repeated observations on the same group of animals were taken into account by including a repeated effect of weaning treatment within batch. Significant day and interaction effects were further analysed using post hoc tests. A Tukey adjustment was used in case of multiple pairwise comparisons. Nursing frequency was analysed with a mixed model including day of observation and batch. Repeated observations were taken into account by including a repeated effect at batch level. Effects of weaning treatment on proportion of piglets with pasty or watery faeces were analysed with generalised linear mixed models using a binomial distribution and logit link function, and including batch as fixed effect. Results are presented as means \pm SEM (based on averages per treatment per batch). $P$-values below 0.05 are considered statistically significant. All data are presented for selected pigs only, with exception of nursing frequency for IS 9 pigs, and feed intake and body weight gain before 9 weeks of age for both IS9 and A4 pigs. IS9 pigs were selected from a total of 24 litters ( 1 sow and her litter were removed from the system because the sow's milk yield was inadequate) and A4 pigs were selected from a total of 23 litters (2 sows died before weaning). Data collected from the 3 litters that were removed from the system were not taken into account in analyses.

\subsection{Results}

\subsubsection{Nursing frequency of IS9 pigs}

The total nursing frequency changed over time for IS9 pigs $(P<0.01$, Figure 6.3). On the second day of IS (day 29), the nursing frequency was lower (17.6 \pm 1.1 vs. $26.4 \pm 1.6$ per 24 hours) than before the start of IS (day 21). Nursing frequency decreased during the period in which sows could voluntarily get away from the piglets, with a lower frequency on day $56(10.1 \pm 2.6)$ compared with day 35 (21.2 \pm 2.3$)$. Until day 35 , all sows still gave successful nursing bouts (ranging from 9 to 28 bouts on day 35). The cumulative percentage of sows that had no successful nursing bouts was $8.3 \%$ on day $42,12.5 \%$ on day 


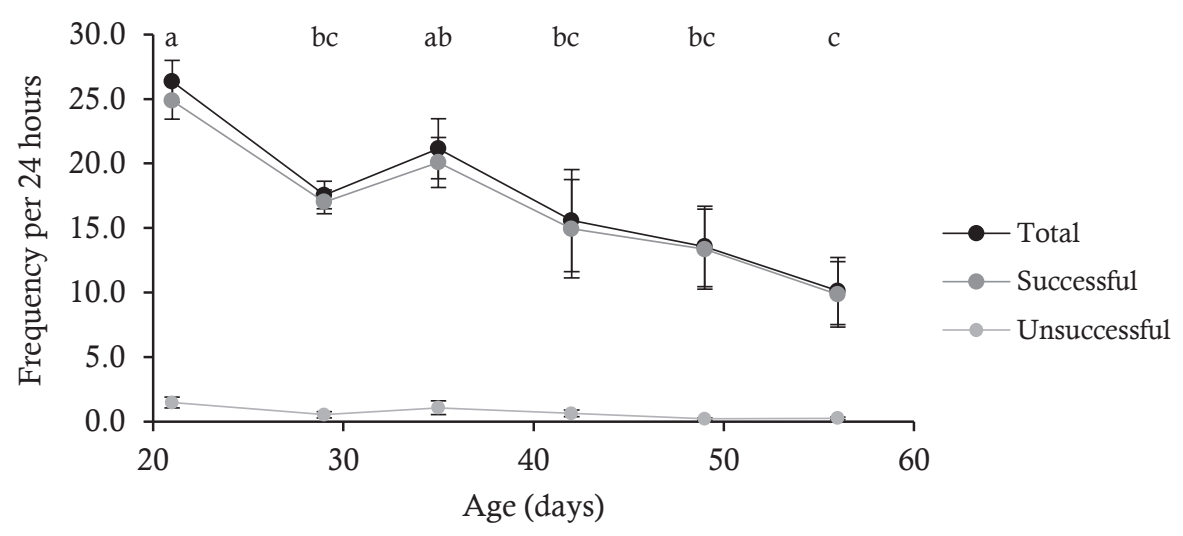

Figure 6.3. Nursing frequency for piglets that were housed in a multi-suckling system. Piglets were subjected to intermittent-suckling from days 28 to 34, during which sows and piglets were separated for 10 hours per day. Thereafter, sows could voluntarily get away from the piglets until weaning on day 63. Data are presented as the frequency of successful nursing bouts (i.e. with milk let-down), the frequency of unsuccessful nursing bouts (i.e. without milk let-down), and the total nursing frequency (i.e. successful + unsuccessful bouts). Values among days lacking a common letter differ significantly (day effect $P<0.01$ ).

49 , and $20.8 \%$ on day 56 . The range in frequency of successful nursing bouts was 0 to 22 on day 56 .

\subsubsection{Feed intake and body weight}

Intake of weaner feed and starter feed was lower in IS9 piglets than in A4 piglets between days 28 to 33 and between days 33 to 63 (Table 6.3). Between days 63 to 68,68 to 98 , and 98 to 126, intake of the first and second grower diet did not differ between treatments. Body weight gain between days 27 to 33 was higher in IS9 piglets than in A4 piglets (Table 6.3). Between days 33 to 63, 63 to 68,68 to 98, and 98 to 126, body weight gain did not differ between treatments. Average body weight was higher in IS9 pigs than in A4 pigs (Figure 6.4). Feed conversion between days 63 to 126 did not differ between IS9 pigs and A4 pigs (IS9: $2.29 \pm 0.03$, A4: $2.24 \pm 0.05$ ). Feed conversion from 4 to 9 weeks of age was not calculated because sow feed intake could not be measured in the IS9 group. 
Table 6.3. Feed intake (weaner, starter, and grower diet) and weight gain of pigs that were raised in a multi-suckling system with differing weaning procedures ${ }^{1}$.

\begin{tabular}{lrrrrrr}
\hline & \multicolumn{2}{c}{ Feed intake per pig, $\mathrm{kg}^{2}$} & & \multicolumn{2}{c}{ Weight gain per pig, $\mathrm{kg}^{2}$} \\
\cline { 2 - 3 } \cline { 6 - 6 } Day & \multicolumn{1}{c}{ IS9 } & A4 & Day & \multicolumn{1}{c}{ IS9 } & \multicolumn{1}{c}{ A4 } \\
\hline & & & 0 to 27 & $5.85 \pm 0.19$ & $5.75 \pm 0.17$ \\
28 to 33 & $0.27 \pm 0.12^{\mathrm{a}}$ & $1.22 \pm 0.18^{\text {b }}$ & 27 to 33 & $1.51 \pm 0.06^{\mathrm{c}}$ & $0.90 \pm 0.08^{\mathrm{d}}$ \\
33 to 63 & $11.43 \pm 0.75^{\mathrm{c}}$ & $22.13 \pm 1.30^{\text {d }}$ & 33 to 63 & $15.54 \pm 0.28$ & $14.37 \pm 0.90$ \\
63 to 68 & $7.32 \pm 0.15$ & $7.29 \pm 0.23$ & 63 to 68 & $3.19 \pm 0.16$ & $3.07 \pm 0.35$ \\
68 to 98 & $50.90 \pm 2.78$ & $48.96 \pm 3.66$ & 68 to 98 & $25.78 \pm 1.80$ & $22.83 \pm 2.48$ \\
98 to 126 & $68.34 \pm 2.98$ & $66.97 \pm 4.02$ & 98 to 126 & $25.80 \pm 1.76$ & $27.70 \pm 1.07$ \\
\hline
\end{tabular}

${ }^{1}$ IS9 pigs were subjected to intermittent-suckling during the fifth week of lactation, followed by a period in which sows could voluntarily get away from their piglets until weaning at 9 weeks of age. A4 pigs were weaned abruptly at 4 weeks of age (day 28) and were relocated to a nursery where they were housed until 9 weeks of age. Pigs from both treatments were relocated to a finishing unit at 9 weeks of age, where they were housed under equal conditions.

${ }^{2}$ Values with a different letter within a line and variable, differ at a significance level of $P$ $<0.05$ (ab) or $P<0.01$ (cd).

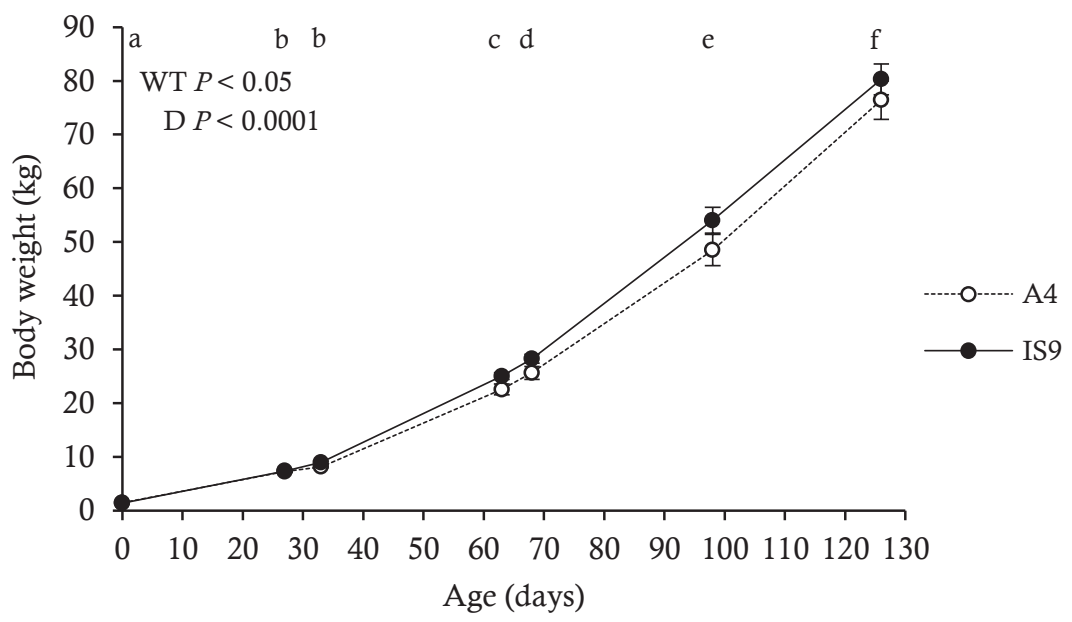

Figure 6.4. Body weight development of pigs that were raised in a multi-suckling system with differing weaning procedures. IS9 pigs were subjected to intermittentsuckling during the fifth week of lactation, followed by a period in which sows could voluntarily get away from their piglets until weaning at 9 weeks of age. A4 pigs were weaned abruptly at 4 weeks of age and were relocated to a nursery where they were housed until 9 weeks of age. Pigs from both treatments were relocated to a finishing unit at 9 weeks of age, where they were housed under equal conditions. $\mathrm{WT}=$ weaning treatment effect. $\mathrm{D}=$ day effect. Values among days lacking a common letter differ significantly. 


\subsubsection{Damage scores}

On day 62, IS9 pigs tended to have lower tail damage scores than A4 pigs (Table 6.4). Ear damage scores and the number of skin lesions on day 62 did not differ between pigs from the different treatments. On day 126, IS9 pigs had lower ear damage scores than A4 pigs. The total number of skin lesions tended to be lower for the IS9 pigs than for the A4 pigs. This difference mainly resulted from fewer superficial lesions; the number of deep lesions did not differ between treatments. Furthermore, fewer lesions tended to be located on the front and middle of the pigs in the IS9 group than in the A4 group. The number of lesions on the rear of the pigs did not differ.

\subsubsection{Faecal consistency scores}

Weaning treatment did not affect the percentage of piglets with at least 1 day of pasty or watery faeces (IS9: $59.7 \pm 5.1 \%$, A4: $80.5 \pm 6.5 \%, P=0.51$; Figure 6.5). IS9 pigs, however, had fewer days with pasty or watery faeces $(0.91 \pm$ 0.12 days vs. $1.54 \pm 0.17$ days, $P<0.01)$. Additionally, IS9 pigs had a lower average faecal consistency score (i.e. more solid faeces) than A4 pigs (0.28 \pm 0.04 vs. $0.45 \pm 0.06, P<0.05)$. The average faecal consistency score was lower

Table 6.4. Scores for damage on ears, tails, and skin at day 62 and 126 of age for pigs that were raised in a multi-suckling system with differing weaning procedures ${ }^{1}$.

\begin{tabular}{lcccccc}
\hline & \multicolumn{2}{c}{ Day $^{5}$} & & \multicolumn{2}{c}{ Day $126^{5}$} \\
\cline { 2 - 3 } \cline { 5 - 6 } & \multicolumn{2}{c}{ IS9 } & A 4 & & IS9 & A4 \\
\hline Ear damage score $^{2}$ & $1.22 \pm 0.12$ & $1.45 \pm 0.19$ & & $0.85 \pm 0.15^{\mathrm{a}}$ & $1.08 \pm 0.19^{\mathrm{b}}$ \\
Tail damage score $^{3}$ & $0.62 \pm 0.15^{\mathrm{x}}$ & $0.81 \pm 0.17^{\mathrm{y}}$ & & $0.80 \pm 0.15$ & $0.79 \pm 0.16$ \\
Total number of skin lesions $^{4}$ & $22.4 \pm 4.0$ & $22.2 \pm 2.1$ & & $16.4 \pm 2.9^{\mathrm{x}}$ & $20.1 \pm 4.1^{\mathrm{y}}$ \\
$\quad$ Superficial skin lesions & $20.0 \pm 3.3$ & $21.0 \pm 2.2$ & & $14.7 \pm 2.7^{\mathrm{x}}$ & $17.7 \pm 3.8^{\mathrm{y}}$ \\
Deep skin lesions & $2.3 \pm 0.8$ & $1.2 \pm 0.3$ & & $1.7 \pm 0.4$ & $2.4 \pm 0.7$ \\
Skin lesions on the front & $10.8 \pm 1.9$ & $10.1 \pm 0.8$ & & $7.6 \pm 0.8^{\mathrm{x}}$ & $9.6 \pm 1.4^{\mathrm{y}}$ \\
Skin lesions on the middle & $8.0 \pm 1.6$ & $9.0 \pm 1.1$ & & $5.4 \pm 1.2^{\mathrm{x}}$ & $6.4 \pm 1.4^{\mathrm{y}}$ \\
$\quad$ Skin lesions on the rear & $3.6 \pm 0.6$ & $3.2 \pm 0.5$ & & $3.3 \pm 1.0$ & $4.1 \pm 1.3$ \\
\hline
\end{tabular}

${ }^{1}$ IS9 pigs were subjected to intermittent-suckling during the fifth week of lactation, followed by a period in which sows could voluntarily get away from their piglets until weaning at 9 weeks of age. A4 pigs were weaned abruptly at 4 weeks of age (day 28) and were relocated to a nursery where they were housed until 9 weeks of age. Pigs from both treatments were relocated to a finishing unit at 9 weeks of age, where they were housed under equal conditions.

${ }^{2}$ Score averaged for both ears. Score ranged from 0 (no damage) to 4 (severe damage).

${ }^{3}$ Score ranged from 0 (no damage) to 3 (moderate damage).

${ }^{4}$ Skin lesions were counted as the number of fresh superficial and deep scratches per body region.

${ }^{5}$ Values with a different letter within a line and day, differ at a significance level of $P<0.05(\mathrm{ab})$ or $P$ $<0.10$ (xy). 


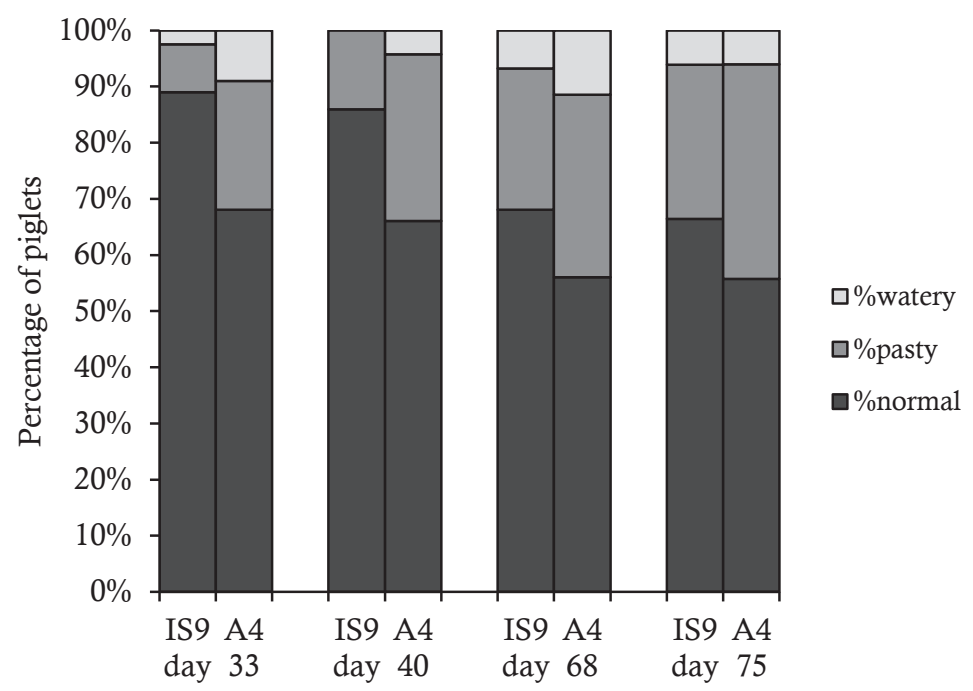

Figure 6.5. Faecal consistency scores on days 33, 40, 68, and 75 of age for pigs that were raised in a multi-suckling system with differing weaning procedures. IS9 pigs were subjected to intermittent-suckling during the fifth week of lactation, followed by a period in which sows could voluntarily get away from their piglets until weaning at 9 weeks of age. A4 pigs were weaned abruptly at 4 weeks of age and were relocated to a nursery where they were housed until 9 weeks of age. Pigs from both treatments were relocated to a finishing unit at 9 weeks of age, where they were housed under equal conditions.

on days 33 and 40 than on days 68 and $75(P<0.01)$.

\subsubsection{Play, damaging oral manipulation, aggression, belly-nosing, and activity}

The total frequency of play behaviour did not differ between pigs from the different treatments (Figure 6.6A). Play frequency, however, declined over time, with levels being higher on days 34 and 41 than on days 69, 76, and 127. When considering separate elements of play behaviour, there tended to be an interaction between weaning treatment and observation day regarding the frequency of individual play (Figure 6.6B). Specifically, on day 34, IS9 pigs showed more individual play than A4 pigs. Moreover, IS9 pigs performed more individual play on day 34 than during the finishing phase and showed more individual play on day 41 than on day 127, whereas A4 pigs showed a 

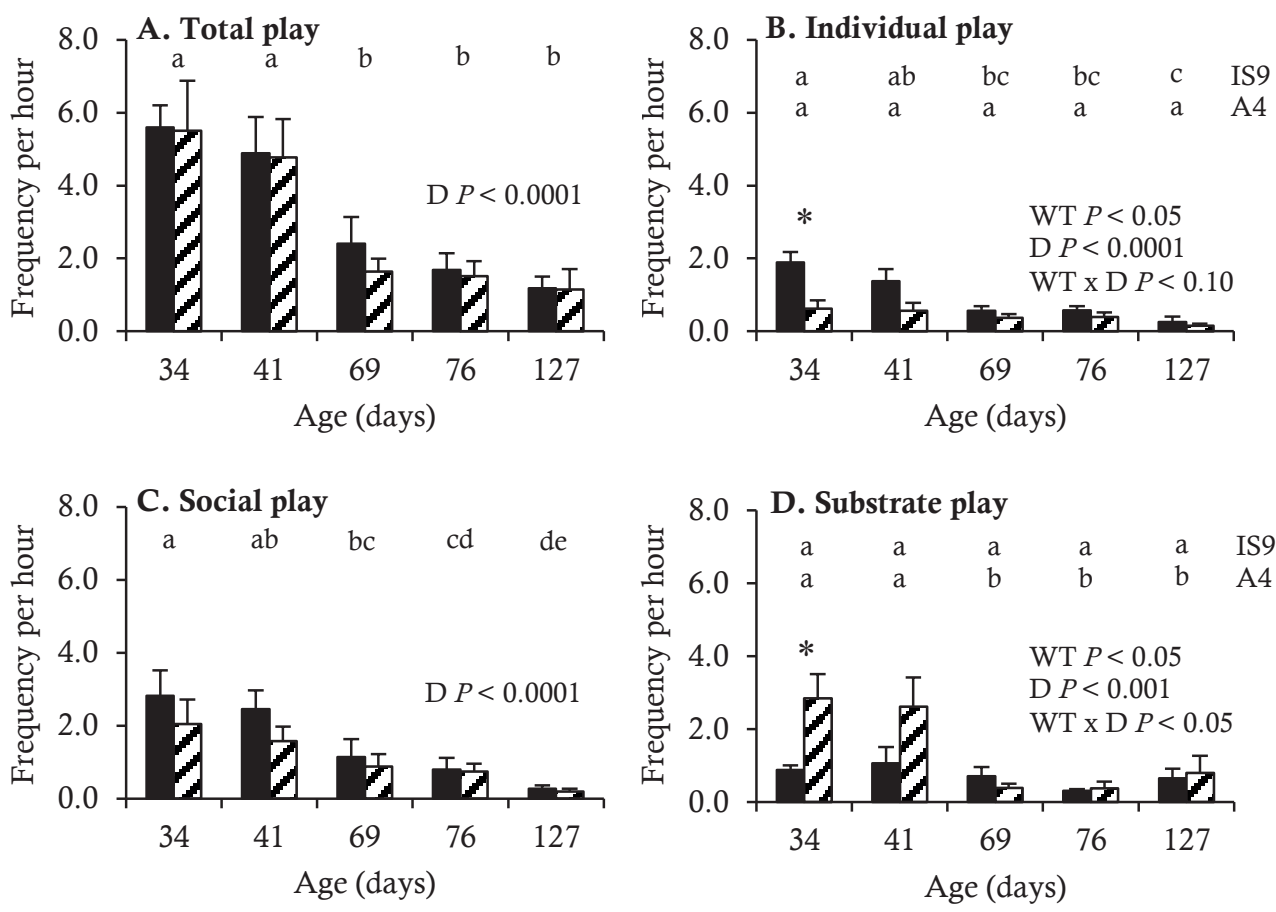

Figure 6.6. Frequency of play behaviour of pigs that were raised in a multi-suckling system with differing weaning procedures. IS9 pigs (solid bars) were subjected to intermittent-suckling during the fifth week of lactation, followed by a period in which sows could voluntarily get away from their piglets until weaning at 9 weeks of age. A4 pigs (dashed bars) were weaned abruptly at 4 weeks of age and were relocated to a nursery where they were housed until 9 weeks of age. Pigs from both treatments were relocated to a finishing unit at 9 weeks of age, where they were housed under equal conditions. WT=weaning treatment effect, $\mathrm{D}=$ day effect, WT $\mathrm{x} \mathrm{D}=$ weaning treatment $\mathrm{x}$ day interaction. Values on days lacking a common letter differ significantly $(P<0.05)$. Values between treatments within a day differ significantly $(P<$ $0.05)$.

similar level of individual play on all observation days. The frequency of social play was unaffected by weaning treatment, but declined over time (Figure 6.6C). An interaction between weaning treatment and observation day affected the occurrence of substrate play (Figure 6.6D). Specifically, on day 34, IS9 pigs performed substrate play less frequently than A4 pigs. Furthermore, IS9 pigs showed a similar substrate play frequency over time, whereas A4 pigs showed more substrate play during the nursery phase than during the finishing phase.

The frequency of damaging oral manipulation was lower in IS9 pigs than in A4 pigs (Figure 6.7A). This treatment effect mainly resulted from a difference in 

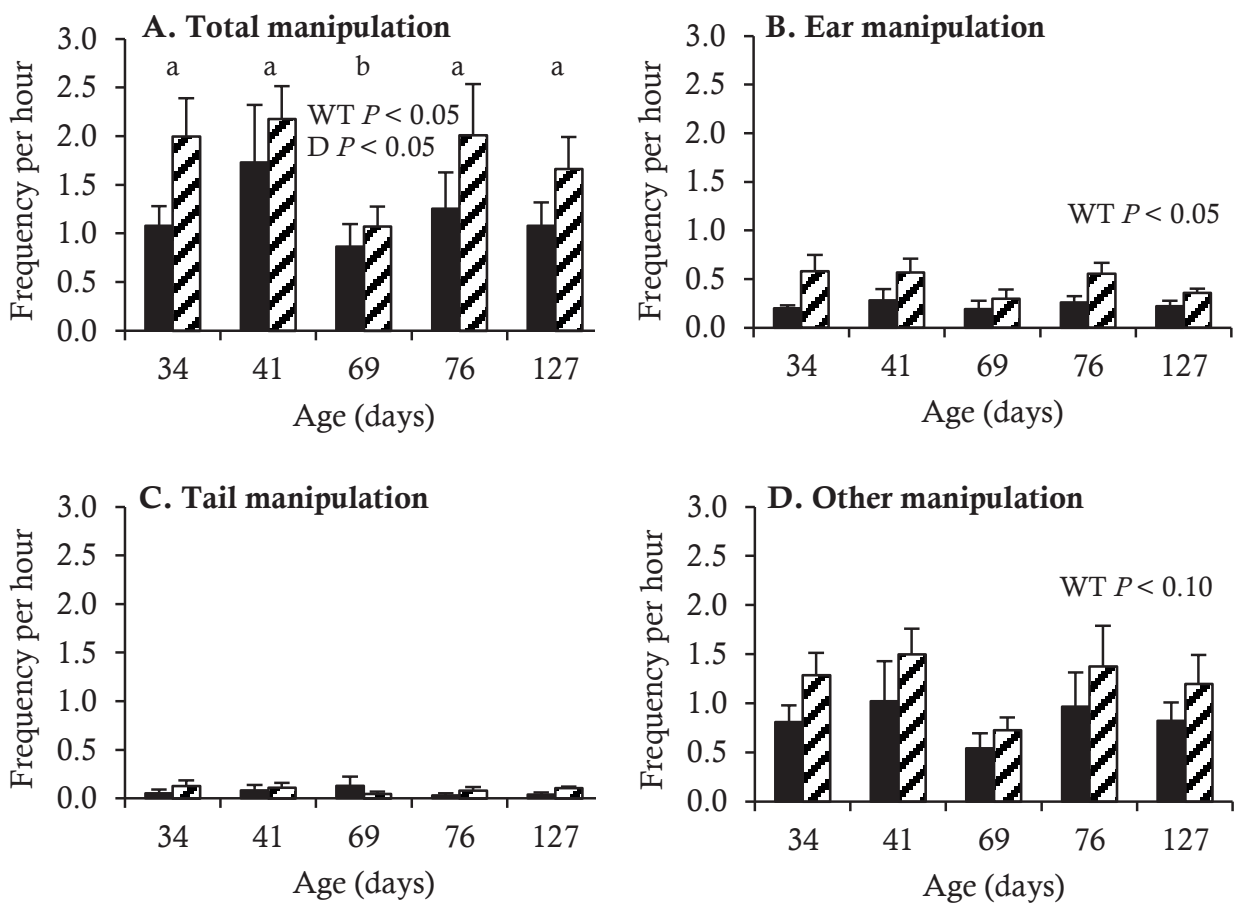

Figure 6.7. Frequency of damaging oral manipulative behaviour of pigs that were raised in a multi-suckling system with differing weaning procedures. IS9 pigs (solid bars) were subjected to intermittent-suckling during the fifth week of lactation, followed by a period in which sows could voluntarily get away from their piglets until weaning at 9 weeks of age. A4 pigs (dashed bars) were weaned abruptly at 4 weeks of age and were relocated to a nursery where they were housed until 9 weeks of age. Pigs from both treatments were relocated to a finishing unit at 9 weeks of age, where they were housed under equal conditions. WT $=$ weaning treatment effect, $\mathrm{D}=$ day effect. Values on days lacking a common letter differ significantly $(P<0.05)$.

ear manipulation (Figure 6.7B) and manipulation of 'other' body parts (Figure 6.7D), as there was no difference in tail manipulation (Figure 6.7C). Overall, there was a lower frequency of manipulative behaviour on day 69 compared with all other observation days.

The frequency of aggression was not affected by treatment, but aggression occurred more frequently on day 41 than on days 69, 76, and 127 (Figure 6.8A). The frequency of head knocking was higher on day 41 than on day 76 (Figure 6.8B) and the frequency of fighting was higher on day 34 than on day 76 (Figure 6.8D). The frequency of biting was affected by an interaction between weaning treatment and observation day. Biting frequency was similar across all observation days for IS9 pigs, but A4 pigs showed more biting on day 

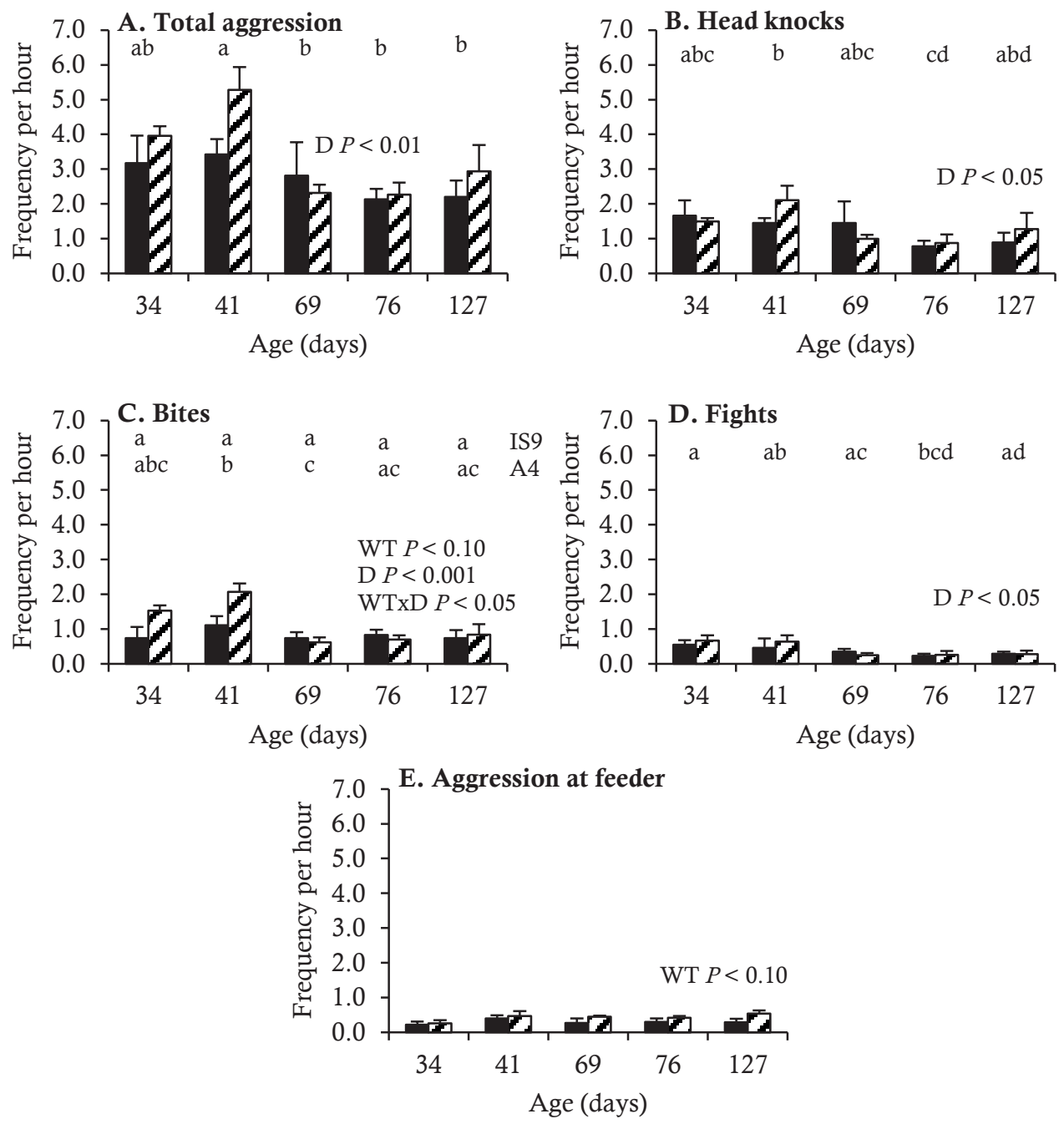

Figure 6.8. Frequency of aggressive behaviour of pigs that were raised in a multi-suckling system with differing weaning procedures. IS9 pigs (solid bars) were subjected to intermittentsuckling during the fifth week of lactation, followed by a period in which sows could voluntarily get away from their piglets until weaning at 9 weeks of age. A4 pigs (dashed bars) were weaned abruptly at 4 weeks of age and were relocated to a nursery where they were housed until 9 weeks of age. Pigs from both treatments were relocated to a finishing unit at 9 weeks of age, where they were housed under equal conditions. WT $=$ weaning treatment effect, $\mathrm{D}=$ day effect, WT x D = weaning treatment $\mathrm{x}$ day interaction. Values on days lacking a common letter differ significantly $(P<0.05)$. 
41 than in the finishing phase (Figure 6.8C). Moreover, IS9 pigs tended to show a lower frequency of aggression at the feeders than A4 pigs (Figure 6.8E).

Belly-nosing was performed less frequently by IS9 pigs than by A4 pigs (Figure 6.9). Additionally, an interaction between weaning treatment and observation day affected the occurrence of belly-nosing. IS9 pigs showed a similar frequency of belly-nosing on all observation days. A4 pigs, however, showed a distinct peak in belly-nosing on day 41 , whereas the level of belly-nosing did not differ on days 34, 69, 76, and 127 .

More IS9 pigs than A4 pigs tended to be active (i.e. scored as 'active' as described in Table 6.2) during observations. Additionally, there was an interaction between weaning treatment and observation day (Figure 6.10). Specifically, on day 34, fewer IS9 pigs than A4 pigs were active. Furthermore, more IS9 pigs were active on day 41 than on days 76 and 127, whereas A4 pigs showed more activity during the nursery phase than during the finishing phase.

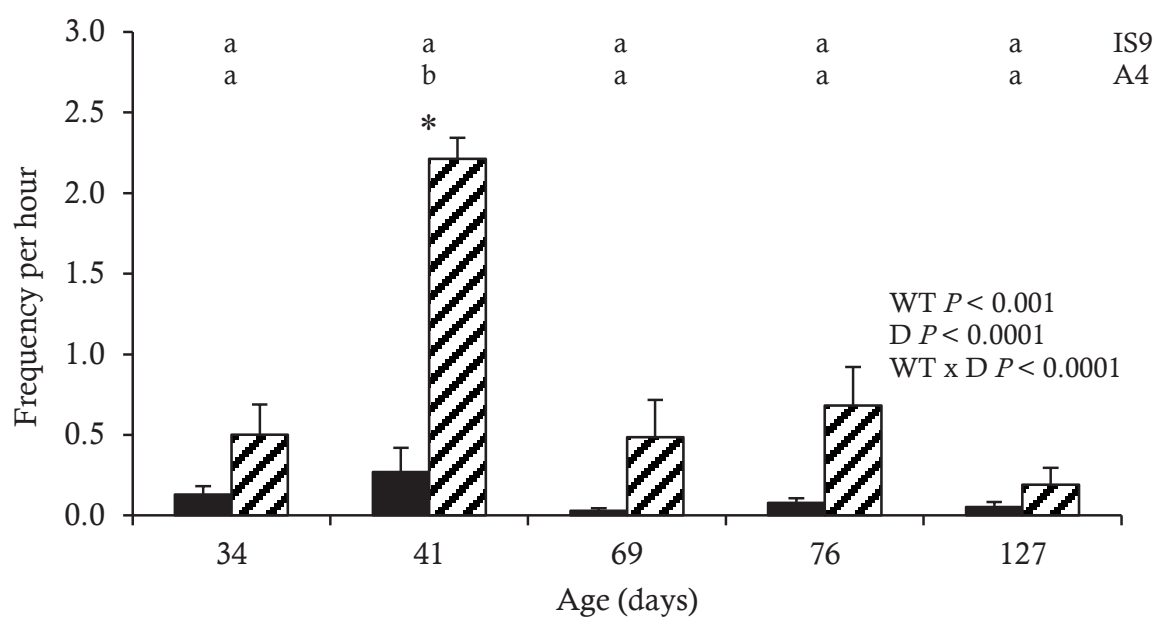

Figure 6.9. Frequency of belly-nosing behaviour of pigs that were raised in a multisuckling system with differing weaning procedures. IS9 pigs (solid bars) were subjected to intermittent-suckling during the fifth week of lactation, followed by a period in which sows could voluntarily get away from their piglets until weaning at 9 weeks of age. A4 pigs (dashed bars) were weaned abruptly at 4 weeks of age and were relocated to a nursery where they were housed until 9 weeks of age. Pigs from both treatments were relocated to a finishing unit at 9 weeks of age, where they were housed under equal conditions. WT = weaning treatment effect, $\mathrm{D}=$ day effect, $\mathrm{WT} \times \mathrm{D}=$ weaning treatment $\mathrm{x}$ day interaction. Values on days lacking a common letter differ significantly $(P<0.05) .{ }^{*}$ Values between treatments within a day differ significantly $(P<0.05)$. 


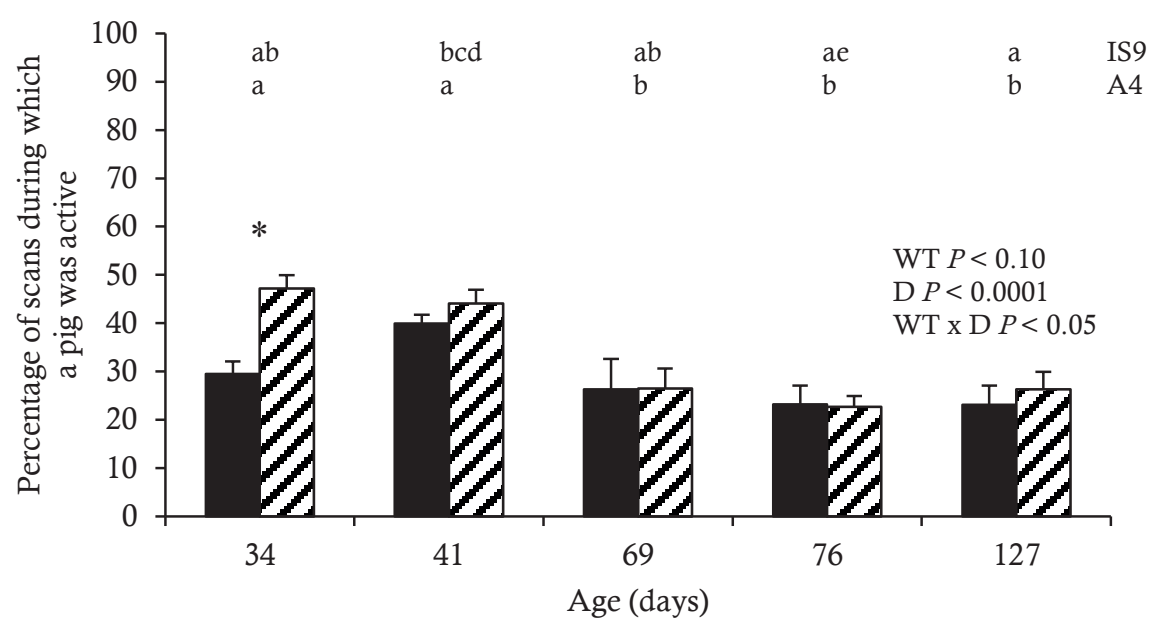

Figure 6.10. Activity of pigs that were raised in a multi-suckling system with differing weaning procedures. IS9 pigs (solid bars) were subjected to intermittent-suckling during the fifth week of lactation, followed by a period in which sows could voluntarily get away from their piglets until weaning at 9 week of age. A4 pigs (dashed bars) were weaned abruptly at 4 weeks of age and were relocated to a nursery where they were housed until 9 weeks of age. Pigs from both treatments were relocated to a finishing unit at 9 weeks of age, where they were housed under equal conditions. WT $=$ weaning treatment effect, $\mathrm{D}=$ day effect, WT $\mathrm{x} \mathrm{D}=$ weaning treatment $\mathrm{x}$ day interaction. Values on days lacking a common letter differ significantly $(P<0.05)$. ${ }^{*}$ Values between treatments within a day differ significantly $(P<0.05)$.

\subsection{Discussion}

This study aimed to investigate effects of 2 different weaning procedures on development of pigs raised in a multi-suckling (MS) system. One group was subjected to gradual weaning, consisting of 1 week of forced IS followed by a period in which sows could voluntarily separate themselves from their piglets until weaning at 9 weeks of age. The other group was weaned abruptly at 4 weeks of age and was subsequently housed in a nursery. At 9 weeks of age, both groups were relocated to a finishing unit. Overall, IS9 pigs seemed to benefit from the more gradual weaning process. Compared with the abruptly weaned A4 pigs, IS9 pigs had a higher body weight gain between days 27 and 33, showed a lower level of belly-nosing, less damaging manipulative behaviour and associated lesions, and a more solid faecal consistency, indicating a lower occurrence of diarrhoea. Both IS and actual weaning did not result in a growth check in IS9 pigs, thus demonstrating a better performance 
around transitions than the A4 pigs. Although differences in production performance disappeared in the finishing phase, some more long-lasting effects remained for e.g. damaging manipulation and belly-nosing.

\subsubsection{The gradual weaning process of IS9 pigs}

To maintain the reproductive output of the IS9 sows, lactational ovulation was stimulated by boar contact during IS in the fifth week of lactation so that sows could be inseminated during lactation. To further stimulate a gradual weaning process, sows were given the freedom to use the IS area voluntarily from the sixth until the ninth week of lactation. To our knowledge, this is the first study describing such a weaning procedure in an MS system. As also recognised by Downing (2015), the combination of an MS system with gradual weaning, including insemination of lactating sows, seems to be a promising management strategy to stimulate piglet development and enhance sow welfare. Regarding the reproductive performance of 24 sows considered in our study; 17 sows showed oestrus during IS and 3 sows showed oestrus during the remaining 4 lactation weeks. Out of the 20 sows that showed oestrus during IS, 19 sows were pregnant following insemination during lactation (also see Laurenssen et al., 2016).

To monitor if piglets were weaned gradually during the 9-week lactation period, nursing frequency was observed. On day 21 , before the start of IS, IS 9 pigs had on average 1.0 successful nursing bouts per hour. Bohnenkamp et al. (2013) reported a successful nursing frequency of 0.7 per hour on day 22 of lactation in an MS system where 6 litters were grouped at 5 days of age. Possibly, the difference in nursing frequency is related to the different layout of the systems. The communal lying area in our study measured $31 \mathrm{~m}^{2}$, providing more space for the sows to nurse their piglets without disturbance, compared with a communal area of $13 \mathrm{~m}^{2}$ that was shared among 6 sows and their piglets in the study of Bohnenkamp et al. (2013). On day 29, the second day of forced IS, the frequency of successful nursing bouts dropped to 0.7 per hour. This is similar to values reported by Berkeveld et al. (2007a). In their study, piglets were subjected to IS by separating them from individually housed sows during 12 hours per day, starting from day 14 of lactation. During the phase in which sows could voluntarily get away from their piglets, nursing frequency gradually declined. The nursing frequencies were considerably higher than in a study by 
Bøe (1993), in which 6 sows could voluntarily leave their piglets to meet in a communal area. The piglets remained in their farrowing pen and were not grouped with other litters. The total nursing frequency in the study of Bøe (1993) was about 0.2 per hour at 5 weeks of age, with all piglets weaned by the sows before 9 weeks of age. In our study, the total nursing frequency decreased from 0.9 to 0.4 per hour on average between 5 and 8 weeks of age. Not all piglets were completely weaned at 9 weeks of age, as $75 \%$ of sows still had milk-producing mammary glands on day 63. Possibly, piglets were weaned earlier in the study of Bøe (1993) than in our study because sows preferred to nurse in the communal area, while the piglets were not able to leave the farrowing pens; piglets under natural conditions leave their nest on average at 9 to 10 days of age and join their mother to integrate with a group of other sows and their litters (Jensen, 1986; Jensen and Redbo, 1987).

In short, our management strategy seemed successful in stimulating a gradual weaning process. Not all piglets were completely weaned at 9 weeks of age, however, and there was variation between sows in nursing frequency. This is consistent with studies in which sows could voluntarily leave their piglets during a 4-week lactation period. In these systems, nursing frequency and use of the piglet-free area were highly variable among sows. Use of the piglet-free area seemed to reflect the sows' investment in their offspring, and did not necessarily indicate a lack of maternal care (Pitts et al., 2002).

\subsubsection{Effects of weaning treatment between weeks 4 and 9}

Forced IS, during which the sows were separated from the piglets during the day, seemed to have less impact for the IS9 pigs than weaning did for the A4 pigs. Apparently, IS9 pigs could maintain their energy intake during IS and did not show a reduction in average daily gain between days 27 to 33 compared with days 0 to 27, like the weaned A4 pigs did. Similarly, Berkeveld et al. (2007b) showed that IS piglets that were separated from individually housed sows for 12 hours per day had a lower growth check after onset of IS (34\% between days 14 to 16) than conventionally housed piglets did after being weaned ( $98 \%$ between days 21 to 23 ). IS9 pigs showed a $68 \%$ higher body weight gain between days 27 to 33 than A4 pigs, despite a lower intake of weaner and starter feed. IS9 pigs likely compensated their lower intake of piglet feed with intake of sow feed and by drinking milk, which was not possible for 
the A4 pigs. The A4 pigs, however, seemed to recover from their growth check, as body weight gain between days 33 and 63 was similar in both groups. Moreover, IS9 pigs numerically had a lower occurrence of diarrhoea; the percentage of IS9 pigs with pasty or watery faeces, averaged over days 33 and 40, was half of the percentage of A4 pigs. Possibly, this is explained by a difference in intestinal morphology; piglets subjected to IS for 10 hours per day during 1 week before weaning at day 26 did not show a significant reduction in intestinal villus height in the first week post-weaning, as was observed in piglets weaned conventionally at day 26 (Berkeveld et al., 2009). Shortened villi are associated with the occurrence of diarrhoea through a reduction in absorptive intestinal capacity (Nabuurs et al., 1993). A4 pigs likely had shorter villi, as a result of weaning, than the IS9 pigs that were still undergoing IS and voluntary separation by the sows.

Besides differences in body weight gain and faecal consistency scores, the A4 pigs' challenge to deal with the post-weaning situation was also reflected in behavioural differences. A4 pigs showed a distinct peak in belly-nosing on day 41, whereas IS9 pigs had a relatively low and constant level of belly-nosing. The A4 pigs' temporal pattern of belly-nosing corresponds with what is reported for piglets weaned before 4 weeks of age; a peak often occurs in the first few weeks after weaning. Belly-nosing may result from frustrated suckling behaviour and the IS9 pigs' low level of belly-nosing may reflect a smoother transition from a milk-based diet to a diet of only solid feed (Widowski et al., 2008). Similarly, piglets that were separated for 12 hours per day from days 14 to 43 rarely performed belly-nosing during IS, whereas around $40 \%$ to $60 \%$ of post-weaning manipulative behaviours consisted of belly-nosing for piglets weaned conventionally at day 21 (Berkeveld et al., 2007a). Furthermore, A4 pigs numerically showed a peak in aggression on day 41, specifically considering biting behaviour, whereas IS9 pigs showed a more constant level of aggression. This may be related to the change in group composition that occurred after weaning of the selected piglets, possibly causing increased levels of aggression to re-establish a dominance hierarchy. Additionally, the increased expression of aggression and belly-nosing, combined with a higher level of damaging oral manipulation, may altogether reflect a more stressed state of the A4 pigs, resulting from weaning (Dybkjær, 1992). Moreover, it was observed that more A4 pigs than IS9 pigs were active on day 34. Possibly this is a reflection of stress-related restlessness due to weaning. Alternatively, A4 
pigs may have been more active as a result of their new environment, as novelty can elicit play behaviour (Newberry et al., 1988; Wood-Gush and Vestergaard, 1991). For instance, in the nursery pen, some toys were available that were not present before weaning, which may have resulted in the higher level of substrate play of A4 pigs on day 34, compared with IS9 pigs. Moreover, the difference in activity may have resulted from the absence of the sows during observations on day 34 in the IS9 group. Berkeveld et al. (2007a) found that, during IS, piglets were more active when the sows were present and less active when the sows were absent during the day, compared with conventionally weaned piglets.

To summarise, the differences in body weight gain, faecal consistency scores, and behaviour between 4 and 9 weeks of age indicate that the A4 piglets were negatively affected by weaning, and that undergoing IS and sow-initiated separation had less profound effects on the IS9 pigs.

\subsubsection{Effects of weaning treatment during the finishing phase and during the entire experiment}

At 9 weeks of age, pigs from both treatments were relocated to a finishing unit. This was the moment of weaning for the IS9 pigs, which seemed to have less impact than weaning had for A4 pigs. There was no decrease in average daily gain between days 63 to 68 compared with days 33 to 63, i.e. the IS9 pigs did not experience a post-weaning growth check. In addition, feed intake of IS9 pigs between days 63 to 68 was at a similar level as for the A4 pigs, despite the IS9 pigs' lower intake of weaner and starter feed before 9 weeks of age. The absence of a growth check and comparable level of feed intake after weaning of the IS9 pigs is likely explained by a more gradual transition to solid feed preweaning in combination with a later weaning age. As found by Berkeveld et al. (2007a), IS piglets showed more eating behaviour during lactation than conventionally housed piglets, and eating behaviour increased over the course of IS. Moreover, IS piglets had a higher average daily feed intake during lactation than conventionally weaned piglets (Kuller et al., 2004; Kuller et al., 2007). As nursing frequency gradually declined between weeks 4 to 9 , IS 9 pigs likely compensated with an increased intake of solid feed. Although IS9 pigs had a lower intake of piglet feed, they likely complemented their solid feed diet with intake of sow feed; a previous experiment showed that MS piglets were 
more frequently in contact with sow feed than with their own piglet feed (van Nieuwamerongen et al., 2015). A gradual decrease in nursing frequency paired with a gradual increase in solid feed intake resembles the weaning process seen in nature, and is important for a successful adaptation of the gastro-intestinal tract to digestion and absorption of solid feed (Miller and Slade, 2003). Additionally, the higher age at weaning may play a role, as maturity of the gastro-intestinal tract is age-dependent. The level of maturation at a conventional weaning age of 3 to 4 weeks generally does not match with a fully solid feed diet (Miller and Slade, 2003). Thus, IS9 pigs may have been more capable of processing solid feed than A4 pigs were at the moment of weaning. The seemingly better transition around weaning was, however, not reflected in the faecal consistency scores, as the percentage of pigs with pasty or watery faeces on days 5 and 12 post-weaning was comparable for IS9 and A4 pigs. Yet, averaged over all days measured, IS9 pigs had fewer days with pasty or watery faeces and had a lower average faecal consistency score (i.e. more solid faeces) than A4 pigs. After the transition phase, feed intake and body weight gain were still similar for IS9 and A4 pigs, and feed conversion over the whole finishing phase did not differ between treatments. Considering the entire experiment, however, IS9 pigs overall had a higher body weight than A4 pigs.

The transition to the post-weaning phase was less reflected in behavioural changes compared with the A4 pigs; IS9 pigs did not show the remarkable peak in belly-nosing and aggression that was observed for A4 pigs 13 days postweaning. For both treatments, the transition to the finishing unit seemed to result in a temporary drop in manipulative behaviour on day 69 , particularly in the A4 pigs. Possibly, this is due to the change in environment, stimulating exploration of the new pen rather than redirection of exploratory drive towards manipulation of pen mates (Studnitz et al., 2007). In the A4 group, the increased space allowance as compared with the nursery pen may play a role. Overall, IS9 pigs showed less damaging oral manipulation than A4 pigs, and more specifically manipulated ears and body parts other than tails and ears less often. Tails were docked, which may explain the comparably low level of tail manipulation in both treatments. In line with the behaviour observations, IS9 pigs had lower damage scores on their ears and tails than A4 pigs. The lower level of damaging behaviour and associated lesions indicate less frustration (Schrøder-Petersen and Simonsen, 2001), and may reflect the smoother transitions around weeks 4 and 9 for the IS 9 pigs, compared with the A4 pigs. 
Other strategies that can ease the transition to the post-weaning situation, such as the presence of a familiar flavour in the weaner pen and facilitation of preweaning eating behaviour by learning from the sow, also decreased the level of manipulative behaviour within the first 2 weeks after weaning (Oostindjer et al., 2011a; Oostindjer et al., 2011b). Interestingly, in our study we found more long-term effects, as e.g. damage scores still differed in the tenth week of the finishing phase. In addition, belly-nosing persisted at numerically higher levels in A4 pigs during the finishing phase, whereas IS9 pigs performed almost no belly-nosing. This may indicate that the second transition was also challenging for the A4 pigs, or that abrupt weaning at an earlier age had long-term effects. Similarly, Gonyou et al. (1998) found that piglets weaned at day 12 continued to perform belly-nosing longer during the finishing phase than piglets weaned at day 21. Lastly, there were no overall differences between IS9 and A4 pigs in the frequency of play and aggressive behaviour. Both groups, however, showed different elements of these behavioural categories -with more individual play and less substrate play and aggression near the feeders in IS9 pigs-, which indicates differences in behavioural development. Despite the similar level of aggressive behaviour, IS9 pigs tended to have fewer skin lesions related to aggression on day 126 than A4 pigs, indicating that aggressive encounters may have been less intense in the IS9 group than in the A4 group. The location of the lesions indicates that aggressive encounters among A4 pigs may have consisted mainly of reciprocal fights -which more frequently result in lesions to the front part of the body-, rather than acts of bullying -which more frequently result in lesions to the rear part of the body- (Turner et al., 2006).

To conclude, IS9 pigs did not show a growth check after weaning, nor behavioural indicators of having difficulty coping with the post-weaning situation. Overall production performance was similar for IS9 pigs and A4 pigs during the finishing phase. The transition to a diet of only solid feed seemed to be more successful for the IS9 pigs than for the A4 pigs, probably due to a more gradual transition to solid feed intake pre-weaning in combination with a later weaning age. Interestingly, the weaning treatments had some longer lasting effects, indicated by overall differences in damaging oral manipulation and belly-nosing, and differences in damage scores during the finishing phase. 


\subsection{Conclusion}

To our knowledge, this is the first study describing a gradual weaning management procedure using forced intermittent-suckling and voluntary sowinitiated separation in a multi-suckling system. Pigs that were weaned with the gradual procedure coped better with the transitions around 4 and 9 weeks of age than pigs weaned abruptly at 4 weeks of age. Additionally, long-term benefits of the gradual weaning procedure were found. To conclude, the combination of a multi-suckling system with a gradual weaning process is promising for improving piglet performance, behaviour, and welfare.

\subsection{Acknowledgements}

The authors gratefully acknowledge the Ministry of Economic Affairs for their financial support (grant number 4417800110) and the staff of Swine Innovation Centre Sterksel for their assistance. Furthermore, the authors want to thank Bjorge Laurenssen, Fleur Bartels, Jan Wijnen, Gisabeth Binnendijk, Caroline Clouard, Inonge Reimert, Claire Walbecque, Anouk Welbers, Sanne Benjamins, Jasmijn Strous, and Eline Burgers for their assistance during various parts of this experiment. Lastly, Henry van den Brand is acknowledged for providing valuable feedback on the manuscript. 


\subsection{References}

Baxter E.M., Lawrence A.B., and Edwards S.A. 2012. Alternative farrowing accommodation: Welfare and economic aspects of existing farrowing and lactation systems for pigs. Animal 6: 96-117.

Berkeveld M., Langendijk P., Bolhuis J.E., Koets A.P., Verheijden J.H.M., and Taverne M.A.M. 2007a. Intermittent suckling during an extended lactation period: Effects on piglet behavior. Journal of Animal Science 85: 3415-3424.

Berkeveld M., Langendijk P., Soede N.M., Kemp B., Taverne M.A.M., Verheijden J.H.M., Kuijken N., and Koets A.P. 2009. Improving adaptation to weaning: Effect of intermittent suckling regimens on piglet feed intake, growth, and gut characteristics. Journal of Animal Science 87: 3156-3166.

Berkeveld M., Langendijk P., Van Beers-Schreurs H.M.G., Koets A.P., Taverne M.A.M., and Verheijden J.H.M. 2007b. Postweaning growth check in pigs is markedly reduced by intermittent suckling and extended lactation. Journal of Animal Science 85: 258-266.

Bøe K. 1993. Maternal behaviour of lactating sows in a loosehousing system. Applied Animal Behaviour Science 35: 327-338.

Bohnenkamp A.L., Meyer C., Müller K., and Krieter J. 2013. Group housing with electronically controlled crates for lactating sows. Effect on farrowing, suckling and activity behavior of sows and piglets. Applied Animal Behaviour Science 145: 37-43.

Downing J.A. 2015. An opportunity to revolutionise sow management. Animal Production Science 55: 1411-1423.

Dybkjær L. 1992. The identification of behavioural indicators of 'stress' in early weaned piglets. Applied Animal Behaviour Science 35: 135-147.

Gonyou H.W., Beltranena E., Whittington D.L., and Patience J.F. 1998. The behaviour of pigs weaned at 12 and 21 days of age from weaning to market. Canadian Journal of Animal Science 78: 517-523.

Jensen P. 1986. Observations on the maternal behaviour of free-ranging domestic pigs. Applied Animal Behaviour Science 16: 131-142.

Jensen P. and Redbo I. 1987. Behaviour during nest leaving in free-ranging domestic pigs. Applied Animal Behaviour Science 18: 355-362. 
Kuller W.I., Soede N.M., van Beers-Schreurs H.M.G., Langendijk P., Taverne M.A.M., Kemp B., and Verheijden J.H.M. 2007. Effects of intermittent suckling and creep feed intake on pig performance from birth to slaughter1. Journal of Animal Science 85: 1295-1301.

Kuller W.I., Soede N.M., van Beers-Schreurs H.M.G., Langendijk P., Taverne M.A.M., Verheijden J.H.M., and Kemp B. 2004. Intermittent suckling: Effects on piglet and sow performance before and after weaning1. Journal of Animal Science 82: 405-413.

Laurenssen B., Strous J., van Nieuwamerongen S., Bolhuis J., van der Peet-Schwering C., and Soede N. 2016. Lactational oestrus in group housed lactating sows. In: 16th International Conference on Production Diseases in Farm Animals, Wageningen, The Netherlands

Miller H. and Slade R. 2003. Digestive physiology of the weaned pig. In: Pluske J., Le Dividich J., and Verstegen M. (eds.) Weaning the pig: concepts and consequences. Wageningen Academic Publishers, The Netherlands.

Nabuurs M.J.A., Hoogendoorn A., Van Der Molen E.J., and Van Osta A.L.M. 1993. Villus height and crypt depth in weaned and unweaned pigs, reared under various circumstances in the Netherlands. Research in Veterinary Science 55: 78-84.

Newberry R.C., Wood-Gush D.G.M., and Hall J.W. 1988. Playful behaviour of piglets. Behavioural Processes 17: 205-216.

Oostindjer M., Bolhuis J.E., Simon K., van den Brand H., and Kemp B. 2011 a. Perinatal flavour learning and adaptation to being weaned: All the pig needs is smell. PLoS ONE 6.

Oostindjer M., van den Brand H., Kemp B., and Bolhuis J.E. 2011b. Effects of environmental enrichment and loose housing of lactating sows on piglet behaviour before and after weaning. Applied Animal Behaviour Science 134: $31-41$.

Pajor E.A., Weary D.M., Fraser D., and Kramer D.L. 1999. Alternative housing for sows and litters 1. Effects of sow-controlled housing on responses to weaning. Applied Animal Behaviour Science 65: 105-121. 
Pitts A.D., Weary D.M., Fraser D., Pajor E.A., and Kramer D.L. 2002. Alternative housing for sows and litters Part 5. Individual differences in the maternal behaviour of sows. Applied Animal Behaviour Science 76: 291-306.

Schrøder-Petersen D.L. and Simonsen H.B. 2001. Tail Biting in Pigs. The Veterinary Journal 162: 196-210.

Studnitz M., Jensen M.B., and Pedersen L.J. 2007. Why do pigs root and in what will they root?: A review on the exploratory behaviour of pigs in relation to environmental enrichment. Applied Animal Behaviour Science 107: 183-197.

Turner S.P., Farnworth M.J., White I.M.S., Brotherstone S., Mendl M., Knap P., Penny P., and Lawrence A.B. 2006. The accumulation of skin lesions and their use as a predictor of individual aggressiveness in pigs. Applied Animal Behaviour Science 96: 245-259.

van Nieuwamerongen S.E., Soede N.M., van der Peet-Schwering C.M.C., Kemp B., and Bolhuis J.E. 2015. Development of piglets raised in a new multi-litter housing system vs. conventional single-litter housing until 9 weeks of age. Journal of Animal Science 93: 5442-5454.

Widowski T.M., Torrey S., Bench C.J., and Gonyou H.W. 2008. Development of ingestive behaviour and the relationship to belly nosing in early-weaned piglets. Applied Animal Behaviour Science 110: 109-127.

Wood-Gush D.G.M. and Vestergaard K. 1991. The seeking of novelty and its relation to play. Animal Behaviour 42: 599-606. 



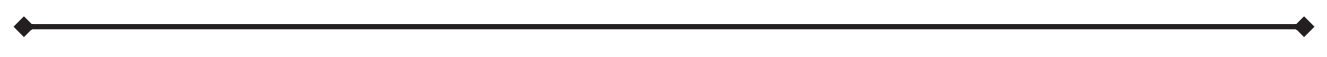

CHAPTER 7

General discussion 


\subsection{Introduction}

The main aim of this thesis was to investigate the development of pigs that were raised in a newly developed multi-suckling (MS) system, with a focus on the transition around weaning and performance later in life. This thesis is part of a larger project with an overall goal to develop a group housing system for sows and their piglets, with the natural behaviour of pigs as the starting point. In this Chapter, the success and risk factors of the key features of the system, related to housing and management, are discussed first. Thereafter, the development of the pigs raised in the MS system is discussed in relation to issues encountered in conventional systems around weaning. Lastly, the practical implementation of the MS system is discussed, not only considering future optimisation of the system itself, but also regarding application of features of the MS system in conventional husbandry.

\subsection{Key features of the MS system}

The MS system consisted of 5 farrowing pens connected to a communal area (Figure 7.1), and environmental enrichment was provided throughout the system. The communal area was divided in an area for general activity and resting, a communal feeding area, and a dunging area. In the second version of the MS system (used in Chapters 4 to 6), an area was included which was only accessible to the sows, to enable investigation of a more gradual weaning procedure. Even though the pigs' natural behaviour was the starting point for developing the system, the practical implementation of its key features was ultimately the result of a compromise with e.g. the system's practicability. To illustrate this, the optimisation processes preceding the use of the 2 systems, regarding design and management, are discussed in this section. As this thesis focusses on piglet development, specifically around weaning and later in life, these aspects will be discussed separately in section 7.3.

\subsubsection{Mimicking a natural social structure}

In our system, the social organisation of pigs in a natural environment, which consists of several sows and their offspring (Gundlach, 1968; Meynhardt, 1980; Jensen, 1986), was mimicked by providing access to a communal area. In this way, sows could maintain social contact with other sows before and after having farrowed individually. The sows housed in our system were familiar 

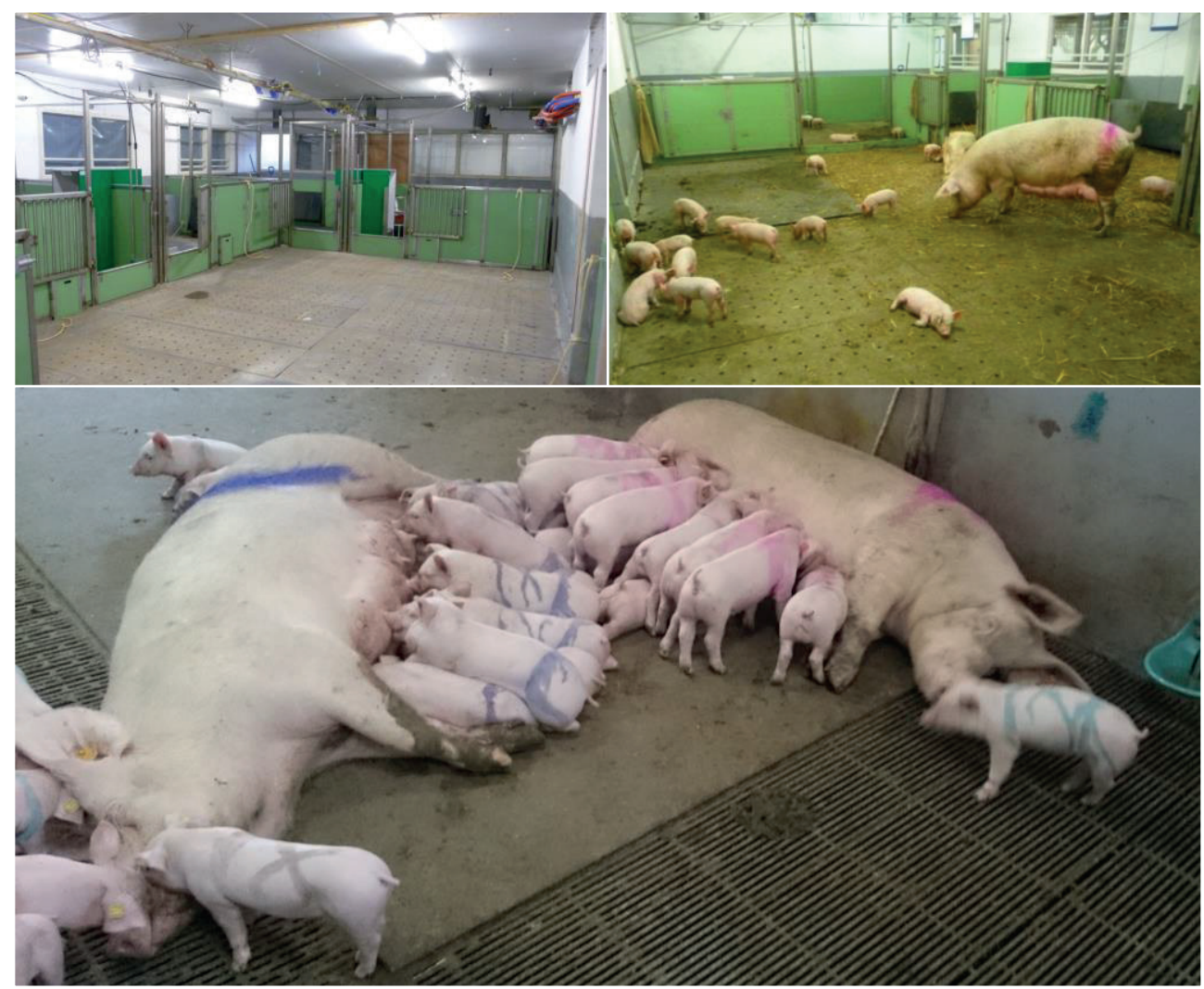

Figure 7.1. The communal area for general activity and resting in the first (upper photos) and second (lower photo) version of the multi-suckling system. The communal area is connected to 5 farrowing pens.

with each other, as they originated from the same group they were housed in during gestation. In nature, the family groups are also formed based on familiarity or relatedness (Meynhardt, 1980; Podgórski et al., 2014). By housing familiar sows in the MS system, aggression and stress during the late stage of gestation due to mixing of unfamiliar animals (see Arey and Edwards, 1998) is prevented, and changes in social environment around the transition to the farrowing unit are reduced, which was suggested to be beneficial in Chapter 2. Overall, we indeed observed little aggression among sows. Moreover, regarding the sows used in Chapter 3, skin damage scores were low at weaning in the MS system, and similar to damage scores of conventionally housed sows ( 0.36 vs. $0.28, P>0.05$, with score 0 defined as no damage and score 1 defined as one or few small scratches, measured on a scale of 0 to 5). 
Conventional farrowing systems provide a large contrast with the, in the EU mandatory, group housing during gestation (Council Directive 2001/88/EC), with an abrupt transition from having freedom of movement and social contact, to confinement in a crate and individual housing around farrowing and lactation. These changes may be stressful for the sows and may e.g. hamper the farrowing process (Yun et al., 2015). Moreover, individual housing may impair sow welfare, specifically later in lactation (Barnett et al., 2001), when sows would have contact with other sows and litters under natural conditions. Our system thus provides opportunities to maintain the social organisation and freedom of movement throughout a sow's productive life and can minimise the need for repeated mixing of unfamiliar animals, which benefits sow welfare (Arey and Edwards, 1998). As housing-related welfare issues may exacerbate over subsequent parities (Broom et al., 1995), these potential benefits may become more manifest on the long term.

\section{Sow-piglet bonding in the first week post-partum}

In our system, the piglets were kept inside their farrowing pen in the first week of life, by maintaining a barrier at the entrance of each farrowing pen (visible in the upper left photo in Figure 7.1), whereas the sows were able to visit the communal area. The piglets were thus initially kept inside their pen before allowing mingling of litters, to facilitate recognition between the sow and her litter, which occurs via olfactory and auditory cues (Meynhardt, 1980; Illmann et al., 2002; Maletínská et al., 2002). In nature, sow-litter bonds appear to be developed within one week after birth (Stangel and Jensen, 1991) and sows initially stay in the nest during the first few days after farrowing. Within the first week post-partum sows start leaving the nest for foraging trips more frequently (Meynhardt, 1980; Stangel and Jensen, 1991). Likewise, in our system, the sows spent the majority of their time with their own piglets in the first week post-partum and only occasionally visited the communal area (>95\% of scan samples, recorded with a 1-hour interval between 9:00 and 15:00 h, was spent in their own farrowing pen on day 5 after farrowing, as observed during optimisation trials of the first version of the system). Around farrowing, and during the first week post-partum, sows would occasionally look inside another farrowing pen, but they did not disturb each other. 


\section{Management in relation to mingling of litters}

When the piglets were about 1 week old, the barriers from the farrowing pens were removed. Thus, the piglets were allowed to access the communal area and other farrowing pens, and could interact with the other sows and litters. In nature, the sow re-joins the family group on average 9 to 10 days after farrowing, after which the litter is gradually integrated in their new social environment (Jensen and Redbo, 1987; Stangel and Jensen, 1991). Mingling litters between 5 and 12 days of age has been recommended, as this may be a sensitive period for socialisation during which mixing may result in limited aggression (D'Eath, 2005; Weary et al., 2008; Kutzer et al., 2009). In other studies, a later grouping age of 3 weeks after farrowing was recommended to e.g. limit stress in sows at grouping (Thomsson et al., 2015; Thomsson et al., 2016). This was, however, in an MS system in which grouping occurred by simultaneously relocating the sows and the piglets from individual farrowing pens to a communal area. This is in contrast to our study, in which sows could maintain social contact before and after farrowing, and relocation of animals did not occur at grouping.

In a conventional system, mingling of litters generally occurs at a later age, i.e. at weaning, for instance to form larger or more uniform groups based on e.g. weight, sex, or number of pigs per pen. This generally results in more aggression and related injuries compared with piglets mingled before weaning. By grouping litters before weaning, as was done in our system, post-weaning mixing of unfamiliar piglets may be unnecessary altogether, thus eliminating one of the simultaneous stressors piglets usually have to cope with at weaning (Weary et al., 2008). Even if unfamiliar piglets raised in the MS system would be mixed post-weaning, this may result in little aggression, as pre-weaning contact with multiple litters has been shown to reduce post-weaning aggression among unfamiliar pigs (Kanaan et al., 2012). A general risk factor of housing pigs in larger groups, however, is that one individual troublesome sow or piglet can affect the whole group, for instance in terms of aggressive behaviour, persistent tail biting, or spread of infection.

We chose to mingle litters by providing access from the farrowing pens to an adjacent communal area, rather than rearranging the farrowing pens to create a communal area, or moving the sows and piglets from their individual 
farrowing pens to a separate multi-suckling room (see Chapter 2 for practical examples of these options). The chosen method may be less stressful, as it limits the number of transitions in physical environment, and it may also limit the occurrence of cross-suckling (see Chapter 2). Although cross-suckling can provide benefits for sows and piglets, it was identified as a risk factor as crosssuckling can result in restlessness and disrupted nursing, with potentially negative consequences for piglet performance. To further prevent these potential risks, the number of piglets per sow was adjusted to the number of functional teats (with a maximum of 14 piglets), as was suggested to be beneficial in Chapter 2. Moreover, the sows had the same date of expected farrowing, as cross-suckling may become problematic with a large variation in age between litters within one group (see Wechsler, 1996). Lastly, we used a group size of 5 sows and their litters, as a relatively small group may facilitate synchronisation of nursing bouts (Illmann et al., 2005), which limits the possibilities for piglets to switch between multiple sows during milk let-down (Maletínská and Špinka, 2001), which lasts only 10 to 20 seconds (Fraser, 1980). Practical reports recommend a group size of maximum 8 sows, to limit cross-suckling (Aubel et al., 2011).

During the optimisation trials, we found that restlessness of sows and piglets around nursing indeed increased after grouping of litters, but more organised nursing bouts resumed after approximately 1 week, with a similar level of restlessness as control animals in a conventional system. Overt aggression resulting in injuries was never observed towards (non-)offspring piglets. Aggressive behaviour towards piglets, such as head knocking or snapping jaws towards a piglet, however, peaked on the day of grouping with a frequency of around 0.3 per hour (also see Olsen et al., 1998). Within 1 week after grouping, the frequency of these behaviours returned to near absence, as was observed before grouping. On average, cross-suckling occurred in about 25\% of successful nursing bouts (i.e. with milk let-down), with on average 0.4 crosssuckling piglets per successful nursing bout. Cross-massaging (i.e. massaging the udder prior to milk let-down by non-offspring piglets) occurred on average in about $50 \%$ of all nursing bouts (i.e. successful and unsuccessful nursing bouts), with on average 0.9 cross-massagers per nursing bout. Anecdotally, the tolerance of cross-suckling differed per sow (observed from their response to non-offspring piglets around nursing). Most sows seemed to be tolerant, but 
some sows, for example, delivered head knocks towards non-offspring piglets when lying in the lateral nursing position.

Likely related to the occurrence of cross-suckling and an increased competition at the udder, we found that piglets in the MS system had a higher average snout damage score on the day before weaning than the conventionally housed piglets (see Chapter 3). In addition, the sows used in Chapter 3 had a higher teat damage score at weaning in the MS system than in the conventional system ( 1.04 vs. $0.79, P<0.05$, with score 0 defined as no damage and score 1 defined as a small lesion $(<1 \mathrm{~cm})$ without damage to the teat canal, measured on a scale of 0 to 3). Moreover, it was observed that the youngest piglets were sometimes forced away from the udder by older cross-suckling piglets (also see Maletínská and Špinka, 2001). Therefore, the age of the youngest litter on the day of grouping was increased from 6 to 8 days, from the first to the second version of the system. Although this did not affect the age difference within one group, the youngest piglets may have been better able to fend for themselves during increased competition at the udder.

Providing sows the possibility to retreat to their farrowing pen seemed beneficial to reduce restlessness. Moreover, it was found to be important to provide sufficient possibilities for piglets to move in and out of the farrowing pens after grouping, to prevent restlessness due to missed nursing bouts. Hence, not only the barriers at the entrance of the farrowing pens were removed, but also additional 'piglet doors' (visible in the upper left photo in Figure 7.1) were removed to prevent sows from blocking access to and from the communal area in case they were lying in front of the farrowing pens. Keeping each farrowing pen intact, rather than rearranging pen components, furthermore provided more possibilities to choose a preferred resting place after grouping; during optimisation it was observed that piglets quickly explored the newly available areas and integrated with other litters, but the preferred lying place (e.g. own farrowing pen, scattered among other farrowing pen(s), or the communal area) differed per day, per litter, and per batch.

Altogether, our experiences confirm cross-suckling as a potential risk factor in the MS system. Nevertheless, we did not experience cross-massaging or crosssuckling as a major issue. For instance, average weaning weight, and the standard deviation of weaning weights, did not differ between piglets from the 
MS system and the conventional system (Chapter 3). Consequences of crosssuckling for individual piglet performance could, however, not be determined in our study due to behaviour observations at litter level.

\subsubsection{Communal feeding}

Another key aspect of our MS system was the communal feeding area (Figure 7.2), where the sows and piglets could eat together. The area consisted of 5 feeding places for the sows, which were separated by horizontal metal bars, surrounded by an area that was only accessible to the piglets. The spacious piglet area contained feeders with piglet feed, and the sows were fed on the floor so that the piglets also had easy access to the sow feed. In nature, foraging is also a joint group activity (Gundlach, 1968; Stolba and Wood-Gush, 1989). Although communal feeding seems beneficial to stimulate piglets' early experience with solid feed (see section 7.3.), it has not often been applied in previously studied MS systems (see Chapter 2). Also in conventional systems, communal feeding is generally not stimulated, as the locations of the sow and piglet feeder are separated, the sow's feeding trough is often not accessible to the piglets, and piglet and sow feeders are too small for synchronised eating.

During the optimisation trials, we observed that some sows were reluctant to eat in the communal feeding area in the first days after farrowing. Therefore, these sows were fed inside their own farrowing pen. Sows may have been reluctant to leave their new-born piglets, as sows in nature stay in close proximity of their nest in the first few days post-farrowing (Meynhardt, 1980; Stangel and Jensen, 1991). In addition, the possibility to feed sows in their individual pen, and the presence of a water nipple, was also found to be important for the rare cases in which a sow had to be kept outside of the group or together with her litter for a longer period of time (e.g. if a sow nursed her litter poorly).

\subsubsection{Defecating and urinating outside the nesting area}

Behind the communal feeding area, the MS system had a separate dunging area (visible on the upper and lower left photo in Figure 7.2) to stimulate urinating and defecating in one designated place, and keep the other communal areas and the farrowing pens clean. In nature, sows eliminate outside the nest (Stolba and Wood-Gush, 1989) and also in our system, the 

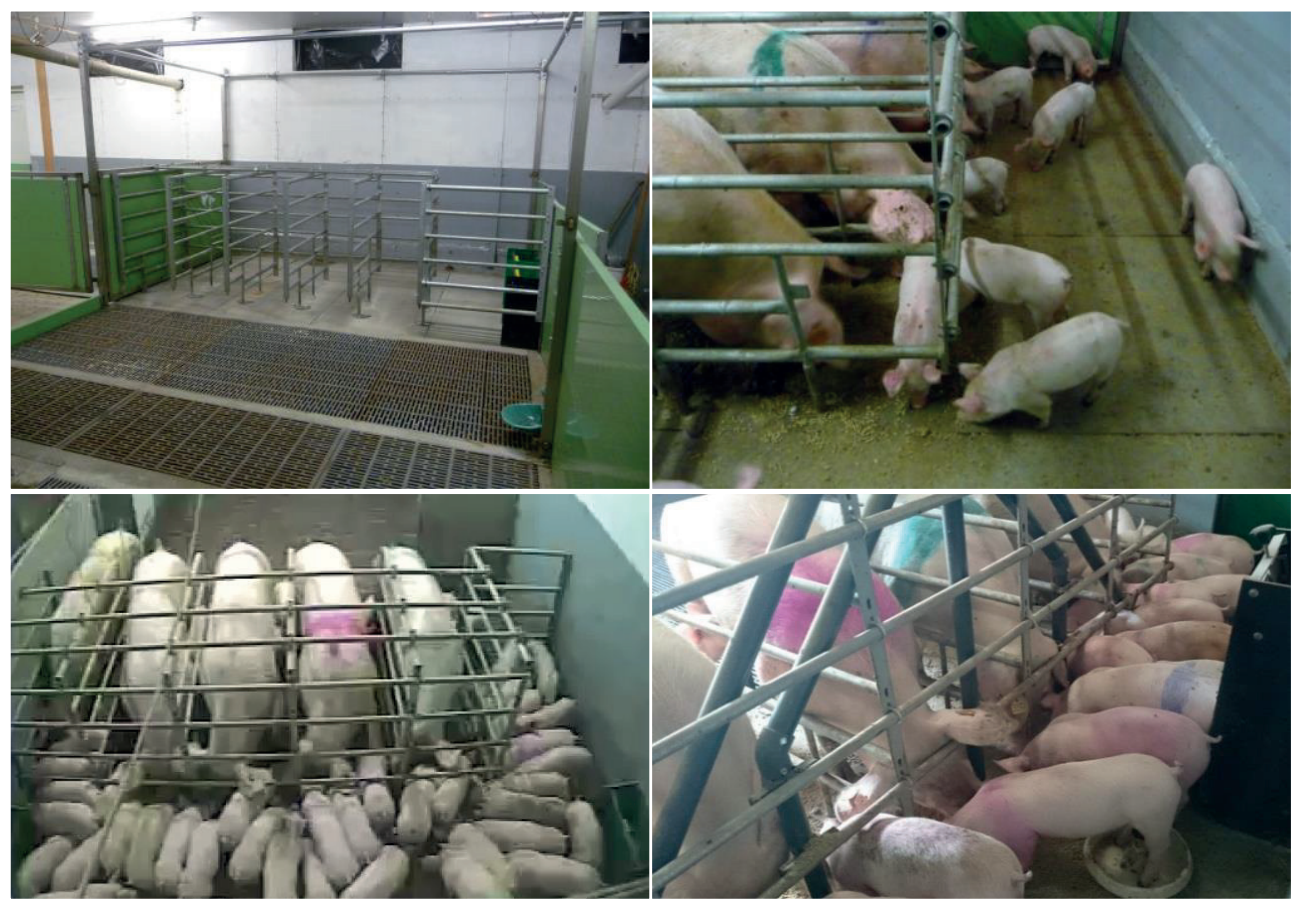

Figure 7.2. The communal feeding area in which sows and piglets could eat together in the first (upper photos) and second (lower photos) version of the multi-suckling system.

sows were able to leave their 'nest' after farrowing to defecate and urinate outside their farrowing pen. In conventional housing, however, sows are confined in such a way that elimination and farrowing occur in the same location. Possibly, this poses a risk for the health of the new-born piglets, as pen hygiene is important in relation to piglet survival (Kirkden et al., 2013).

During the optimisation trials in the first version of the MS system, it was observed that sows often did not go to the dunging area in the first days after farrowing, but rather stepped out of their farrowing pen and eliminated in front of it. This was likely related to the possible reluctance of the sows to leave their new-born piglets (Meynhardt, 1980; Stangel and Jensen, 1991). Also during the first weeks of lactation, however, pen fouling remained an issue and sows sometimes used the farrowing pens of other sows to eliminate. In general, 2 corners of the resting area were most fouled (see Figure 7.1; the corner where 2 farrowing pens adjoined on the upper left photo, and the corner covered by the mat on the upper right photo). Several attempts were made to reduce pen fouling, using insights from another study conducted at the used research 
facilities, which focussed on pigs' eliminative behaviour (Plomp et al., 2016). Examples include adding a light source in the dunging area, adding extra partitions in the dunging area, and indicating the dunging area by making the floor of the dunging area wet before the sows entered the system. These adjustments had limited success. In the second version of the system, however, pen fouling was not an issue. This was likely related to the use of slatted flooring directly in front of all farrowing pens and in the corners of the resting area.

\subsubsection{A more gradual weaning process during an extended lactation}

An intermittent-suckling (IS) area, accessible only to the sows, was added to the second version of the system (Figure 7.3) to enable a more gradual weaning process during an extended lactation period of 9 weeks. Gradual weaning was stimulated by a combination of forced intermittent-suckling in the fifth week of lactation, followed by voluntary use of the IS area by the sows. The piglets were prevented from entering the IS area by a barrier that the sows could step over (Figure 7.4). To direct the sows to move to and from the IS area, sows were fed in the IS area in the mornings and in the indoor communal feeding area in the afternoons, from the start of forced IS until week 9. During the period of forced IS, the sows were separated from the piglets for 10 hours daily by bringing the sows to the IS area where also a boar was present (Figure 7.3, right photo). This approach also aimed to stimulate lactational oestrus and ovulation, so that sows could be inseminated during lactation, thereby attempting to maintain the yearly reproductive output of the sows despite the extended lactation period (see Gerritsen et al., 2008).
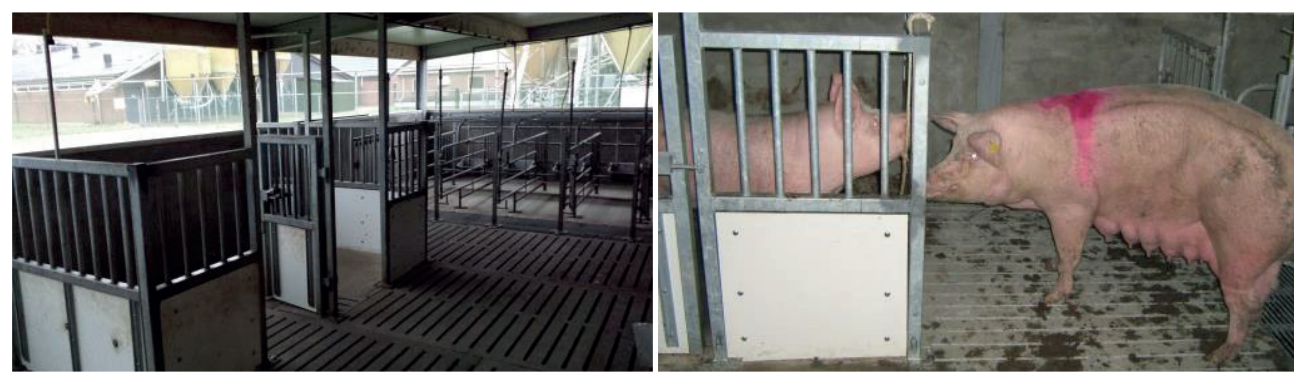

Figure 7.3. The covered outdoor intermittent-suckling area, which was accessible only to the sows. During the fifth week of lactation, the sows were housed in the intermittent-suckling area for 10 hours daily and could contact a boar (right photo). From the sixth until the ninth week of lactation, the sows could use the intermittent-suckling area voluntarily (the boar was absent in this period). 


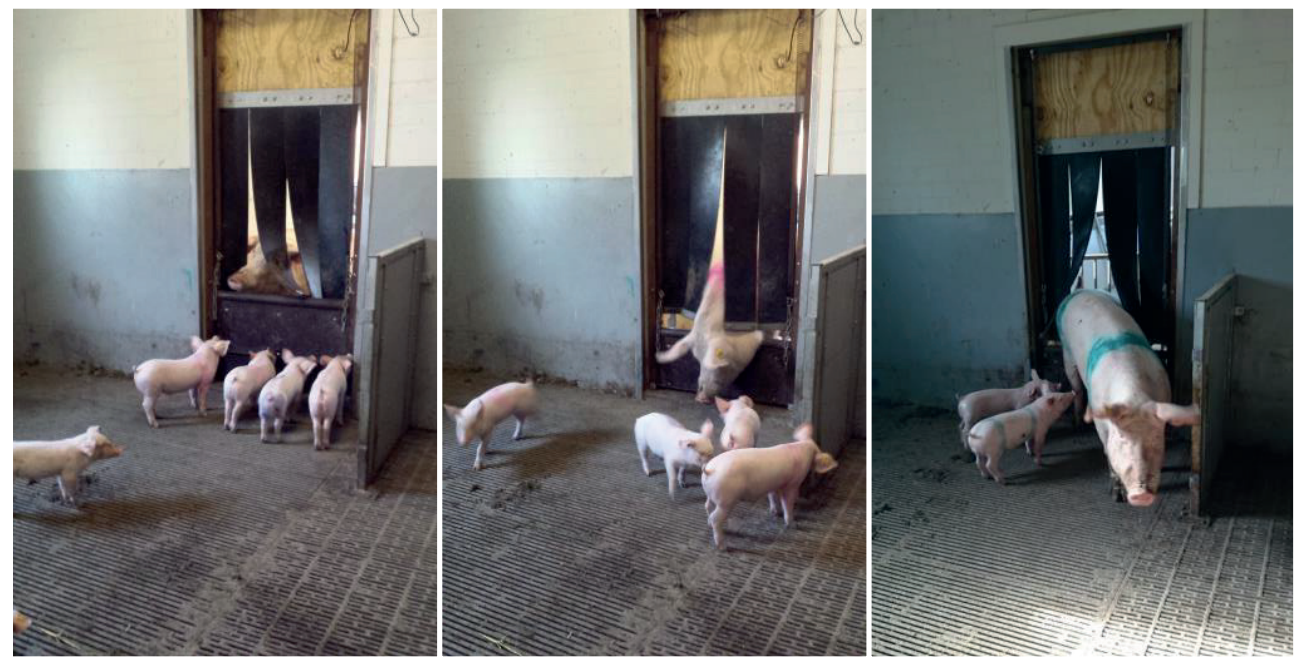

Figure 7.4. Passage between the intermittent-suckling area (accessible only to the sows) and the communal area of the multi-suckling system.

A risk of lactational oestrus in an MS system may be an increase in mounting behaviour, and possibly restlessness and aggression, among sows around oestrus (Soede and Kemp, 1996). In addition, forced separation during IS may cause restlessness among sows and piglets. After the sows' return to their piglets during the period of forced IS, restlessness may jeopardise the piglets by e.g. increasing the risk of trampling. Little is known from literature, however, about these potential risks in MS systems in which lactational oestrus is stimulated. In the first optimisation trials, we found that both sows and piglets were restless after separation and we installed radios in the IS area and in the indoor MS unit to limit auditory contact between sows and piglets during separation. During the period of forced IS, we observed little aggression (a frequency per hour of $1.5 \pm 0.2$, mainly consisting of head knocking and biting) and mounting behaviour $(0.5 \pm 3.0$ occurrences per hour $)$ among sows in the IS area. Also after the sows had returned to their piglets, the frequency of aggression among sows was low (on average $0.13 \pm 0.04$ per hour) and mounting behaviour rarely occurred. After the sows returned, piglets were generally restless and crowded among the sows. Sows would occasionally push piglets out of their way (included in aggressive behaviours towards piglets, which occurred at a frequency per hour of $0.18 \pm 0.05$ towards offspring and $0.51 \pm 0.11$ towards non-offspring), but it was rarely observed that this resulted in a dangerous situation, such as a piglet being stepped on by a sow. 
Although the combination of boar contact and reduced contact with the piglets has previously been found effective to induce lactational oestrus (Mota et al., 2002; Kongsted and Hermansen, 2009; van Wettere et al., 2013), asynchronous or unpredictable onset of oestrus is a potential risk factor, and success of induction may depend on e.g. sow parity (Soede et al., 2012). Synchronisation of oestrus is important in relation to batch farrowing on farms, to avoid variation in farrowing dates. In MS systems, this may for instance increase the risk of cross-suckling, as previously indicated (also see Wechsler, 1996). Of the sows that were subjected to the gradual weaning treatment in Chapter $6,83 \%$ showed oestrus. Including data from the optimisation trials, sows showed oestrus on average $5.5 \pm 1.4$ (range 3 to 9) days after the start of forced IS and $48 \%$ of those sows came in oestrus on the fifth day of IS.

Several factors were found to be important to induce lactational oestrus and ovulation in the sows. Firstly, it seems that sows should be in good condition prior to the start of forced IS, as sows that showed lactational oestrus had more backfat at the start of IS $(14.0 \pm 0.6 \mathrm{~mm}$ vs. $11.5 \pm 0.9 \mathrm{~mm}, P<0.01)$ than sows that did not show lactational oestrus. Furthermore, it is important that a sexually mature boar is used to stimulate the sows (Kemp et al., 2005). In the first optimisation trial, however, only a sexually immature boar was available at the research farm, and in this trial only $40 \%$ of the sows showed lactational oestrus. Thereafter, a mature boar was used. In the next three optimisation trials, the percentage of sows showing lactational oestrus varied between 80 and $100 \%$. During the first batch used in Chapter 6, however, none of the sows responded to forced IS (i.e. no lactational oestrus was shown). One contributing factor may be that the radios were accidentally not switched on during this batch. This may give an indication that the radios were not only important to reduce restlessness among sows and piglets during separation, but that this may also be important in relation to the responsiveness of the sows to forced IS. In addition, it should be noted that the radios were not yet present in the first optimisation trial, which may also have contributed to the low occurrence of lactational oestrus in that batch, besides the use of an immature boar.

Because the obtained results were variable thus far, the management of the boar during forced IS was adjusted. In the preceding trials, the boar was continuously present in the boar pen in the IS area. This may, however, result 
in habituation of the sows to the boar (Kemp et al., 2005), which may limit effectiveness of boar exposure in induction of lactational oestrus. The final protocol for boar management was characterised by repeated introduction of the boar. Moreover, the boar was allowed to interact freely with the sows outside the boar pen, in the IS area of the sows. The protocol consisted of continuous presence of the boar in the boar pen in the IS area during days 1 and 2 of forced IS, combined with physical contact with the sows twice daily for 1 hour each time. On days 3 and 4, the boar was in its pen in the IS area for 3 hours in the morning and was housed elsewhere (out of direct contact with the sows) during the remaining time. During days 5 to 7 , the boar was housed in its pen for 3 hours in the morning and 3 hours in the afternoon and was housed elsewhere during the remaining time. In the last 4 batches run in the system, all sows showed lactational oestrus.

Insemination during lactation can result in similar pregnancy rates, farrowing rates, and litter sizes compared with sows inseminated after weaning (Soede et al., 2012). Of the sows used in Chapter $6,95 \%$ of the sows that showed oestrus ovulated and were inseminated during lactation (between days 28 to 35). The subsequent farrowing rate was $89 \%$ (1 sow died in the gestation unit and 1 sow was culled due to repeat breeding), with on average 16.5 live born piglets and 1.2 stillborn piglets (also see Laurenssen et al., 2016 for data including optimisation trials). Thus, overall, satisfactory results were obtained regarding the reproductive performance of the sows during the last trials run in the second version of the system.

Lastly, during the optimisation trials of the second system, we experimented with weaning ages of 6 and 8 weeks. Finally, we decided to wean the piglets at 9 weeks of age because this provides a better moment to relocate the sows to the gestation unit, as mixing with unfamiliar sows before the critical period of implantation of the embryos may impair sows' reproductive performance (Arey and Edwards, 1998). Moreover, by weaning the piglets at 9 weeks of age, they could be relocated from the farrowing unit to the finishing unit, thus eliminating the extra relocation to a nursery, in which piglets are conventionally housed between 4 and 9 weeks of age. Hence, an additional change in environment, and associated stress (Bøe, 1993), was avoided. 


\subsubsection{Space and environmental enrichment}

A general aspect of the MS system was the provision of more space and enrichment materials, as compared with conventional housing. In nature, pigs have varied and complex surroundings as they live in home ranges up to 35 $\mathrm{km}^{2}$, consisting of diverse habitats (Singer et al., 1981; Dardaillon, 1986; Russo et al., 1997; Dexter, 1999; Saunders and McLeod, 1999). In our system, enrichment consisted of 5 hessian sacks and 5 ropes in the communal area, and in each farrowing pen, 1 handful of long-stemmed straw was provided twice daily from day 2 post-partum onwards. Space allowance and the use of enrichment were restricted by the dimensions of the existing buildings and the slurry management system, respectively. The relevance of the availability of space and nest building material specifically in relation to nest building and maternal behaviour will be discussed later in this section.

The sows were continuously given freedom of movement before, during, and after farrowing. Together with the availability of enrichment and social contact with other sows and litters, this may reduce stress and improve welfare as compared with a conventional system in which a sow is continuously confined in a relatively barren environment (Barnett et al., 2001). Especially in late lactation, sows may be bothered by the continuous presence and close proximity of the piglets (Cronin et al., 1991; Jarvis et al., 2006). In line with this, during optimisation of the second version of the system, we observed that sows housed in farrowing crates showed aggression towards piglets 6 to 7 times more frequently on day 27 of lactation than sows in the MS system. In addition, as a possible indication of better welfare in the MS system (Mason and Latham, 2004), we found that sows spent a lower percentage of the observed time on performing stereotypical behaviour (i.e. sham chewing or bar biting) than in the conventional system $(0.5 \pm 0.2$ vs. $8.0 \pm 1.0 \%, P<0.05,168$ hours of observation in total, divided over 6 days). Also, sows in the MS system occasionally showed play behaviour (i.e. substrate play or locomotor play), which is often used as an indicator of good welfare (Held and Špinka, 2011). Play behaviour was shown at a frequency of 0.03 occurrences per hour, and was not observed in the conventional system (due to a lack of space and available substrate). 
Lastly, related to both sow and piglet welfare, a risk factor was encountered in our system, which was not addressed in Chapter 2. As the sow has no designated space to which she is confined, there was a compromise in suitability of the flooring for both sows and piglets. We used concrete and metal flooring throughout the MS system to provide sufficient grip for the sows, in order to prevent slipping and injuries. Such flooring may, however, be too abrasive for the piglets. During the optimisation trials of the first system, a plastic mat was added to each farrowing pen to prevent injuries (e.g. see $\mathrm{Gu}$ et al., 2010), which may occur e.g. when the piglets' front paws scrape across the floor during suckling. Correspondingly, we monitored lesions on piglets' carpal joints (see Chapter 3) and found no difference in lesion scores at weaning compared with the conventionally housed piglets. Nevertheless, the percentage of piglets that received veterinary treatment was numerically about 4 times higher in the MS system than in the conventional system, which was mainly attributed to lameness. Possibly, this was not reflected in the lesion scores, as the prevalence and severity of carpal lesions may decline after 10 days of age (Zoric et al., 2004; Zoric et al., 2009). The issue persisted in the second version of the system, in which around $17 \%$ of piglets received veterinary treatment, again mainly attributed to lameness. Hence, suitability of flooring remained a point of attention.

\section{Nest building and maternal behaviour}

Our system had 5 farrowing pens in which sows had space to turn around, had nesting material available, and could farrow individually (Figure 7.5). These aspects are, however, not necessarily features of group housing systems for lactating sows in general. For example, approximately $30 \%$ of the studies reviewed in Chapter 2 incorporated a farrowing crate. We included individual 'loose' farrowing pens with nesting material in our system to enable the expression of nest building behaviour (Yun et al., 2014) and provide some form of isolation from the other sows during farrowing. Likewise, in a natural environment, a sow separates from the family group to find a suitable site to build a nest in which she farrows (Gundlach, 1968; Meynhardt, 1980; Jensen, 1986; Jensen et al., 1987; Stolba and Wood-Gush, 1989; Petersen et al., 1990; Stangel and Jensen, 1991; Jensen et al., 1993). In contrast, in conventional farrowing systems, sows have limited possibilities to express nest building behaviour due to insufficient space to turn around and a lack of suitable nesting 


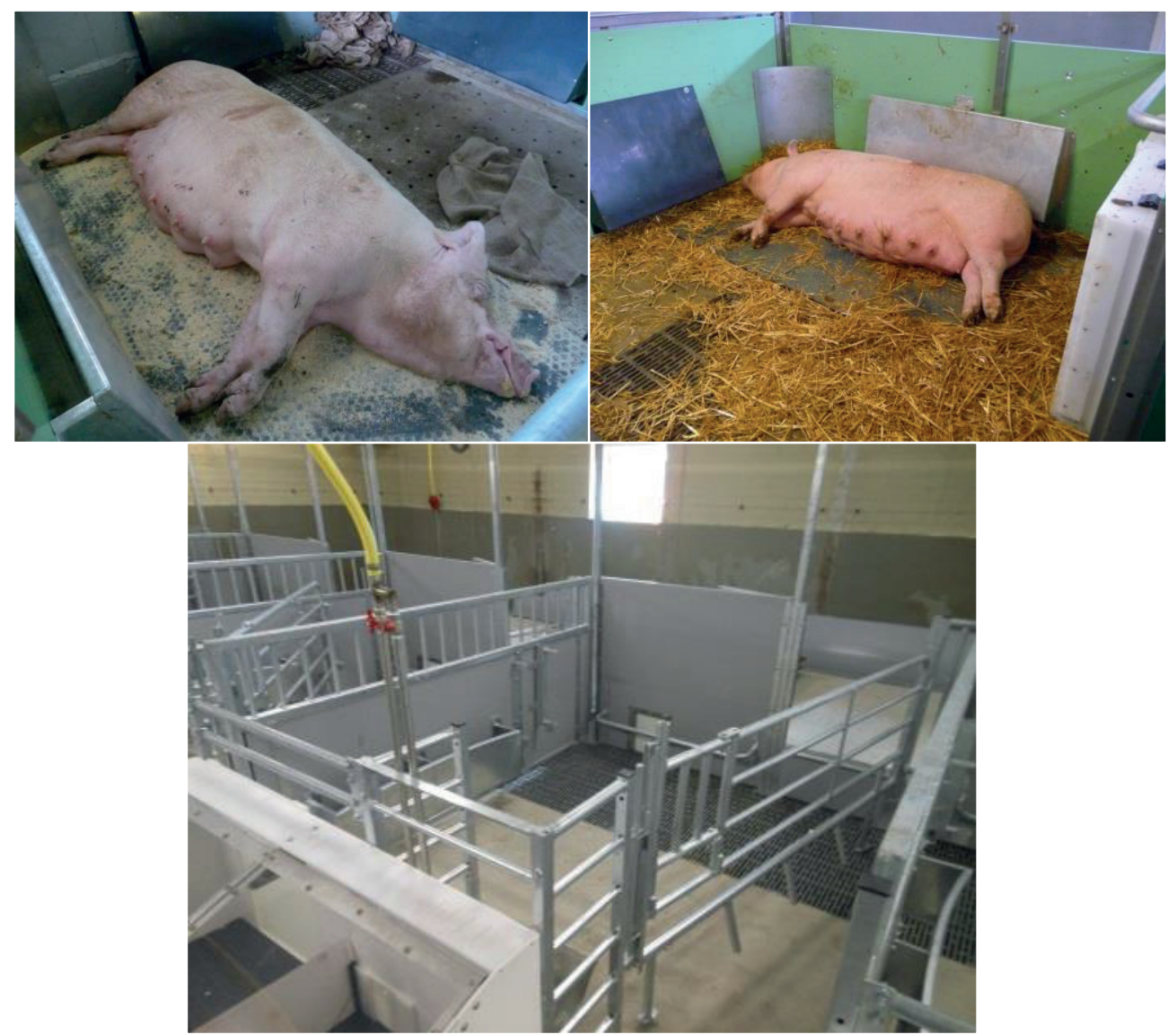

Figure 7.5. Loose individual farrowing pens in the first (upper photos) and second (lower photo) version of the multi-suckling system.

material. Domestication has not eliminated the need to perform nest building behaviour (Gustafsson et al., 1999) and restricting this behaviour can impair sow welfare, maternal behaviour, and piglet survival (Wischner et al., 2009; Yun and Valros, 2015). Moreover, expression of maternal behaviour is also restricted in a conventional system during subsequent lactation, as free interaction between the sow and her litter is prevented. In line with this, in sows housed in an MS system Grimberg-Henrici et al. (2016a) observed a stronger behavioural response to piglet distress calls and to separation from, and reunion with, their piglets in their home pen compared with conventionally housed sows. The latter, however, showed a more distinct behavioural response than the MS sows when the same tests occurred in a separate testing arena, rather than in the home pen. As maternal defensiveness 
may result in human-directed aggression (Baxter et al., 2011a), the possibility to separate the sows from the piglets was found to be important in our system, to ensure safety of the stockpersons. For instance, occasionally, a sow was aggressive towards humans in the first days after farrowing. In the second version of the system, possibilities to separate the piglets from the sows within each farrowing pen were improved, by use of piglet nests in which piglets could be locked automatically. Also, the piglet nests were positioned at the front of each farrowing pen (see Figure 7.5, lower photo), and were thus accessible from the corridor (i.e. without having to step into the farrowing pen). This also eased management during standard procedures, such as ear tagging.

In our system, the use of nesting material was adjusted during the optimisation trials of the first system. First, $2 \mathrm{~kg}$ of long-stemmed straw and 3 hessian sacks were provided per farrowing pen (Figure 7.5, upper right photo), but this was changed to 5 hessian sacks per pen (Figure 7.5, upper left photo), due to a combination of incompatibility of straw with the slurry management system and inexperience of stockpersons with using straw on the research farm. Ultimately, the used nesting material may not have been optimal to stimulate nest building behaviours of the sows and ensure termination of nest building well in advance of farrowing, even though more material was provided than in a conventional system. For instance, minimum requirements for nesting material, as recommended by Baxter et al. (2011b), include availability of $2 \mathrm{~kg}$ of long-stemmed straw, branches, and bedding material. In addition, Westin et al. (2015) reported that provision of 15 to $20 \mathrm{~kg}$ of chopped straw 2 days prior to farrowing may be more effective in stimulating nest building behaviours than daily provision of smaller quantities of straw (i.e. 0.5 to $1 \mathrm{~kg}$, and $2 \mathrm{~kg}$ close to farrowing).

\section{Piglet mortality in relation to loose farrowing}

A possible risk factor of providing sows more freedom of movement is a higher level of piglet crushing, compared with conventional housing in farrowing crates (Weber et al., 2007; Wechsler and Weber, 2007). During the optimisation trials, we made some adjustments in management and farrowing pen design which aimed to limit piglet crushing. The adjustments mainly aimed to guide the lying behaviour of the sows and piglets to prevent (near-)crushing situations. In the final version of the first system, the piglets 
were stimulated to use the piglet nest as quickly as possible after birth, as the first 24 hours after farrowing pose a great risk for crushing (Marchant et al., 2001). This was done by placing hessian sacks that were used for nest building, i.e. with the sow's odour, in the piglet nest and locking the piglets inside the nest during the first 2 feeding bouts of the sow post-farrowing. In addition, the piglet nests were heated to attract the piglets away from the sow's body warmth. Moreover, the sows were stimulated to lie with their back against the wall in front of the piglet nest, to create a lying location that was predictable for the piglets. In addition, sloped walls were installed to provide piglets room to escape when a sow would lie down against a wall. And, lastly, litter sizes were equalised, with a maximum of 14 piglets per sow, as the risk of crushing increases with litter size (Weary et al., 1998; Weber et al., 2009).

During optimisation of the first system, we found that a transition from ventral to lateral recumbency was the most dangerous postural change in relation to crushing, as it resulted in the death of $62 \%$ of all crushed piglets. Moreover, $64 \%$ of all crushed piglets were crushed in the middle of the farrowing pen, i.e. when the sow lay down without support of a wall. Other studies have also found rolling-over behaviour and unsupported lying-down movements to be high-risk behaviour for piglet crushing, especially if the movements are quick or uncontrolled (Wechsler and Hegglin, 1997; Damm et al., 2005; Burri et al., 2009; Danholt et al., 2011). Therefore, in the second version of the system, the
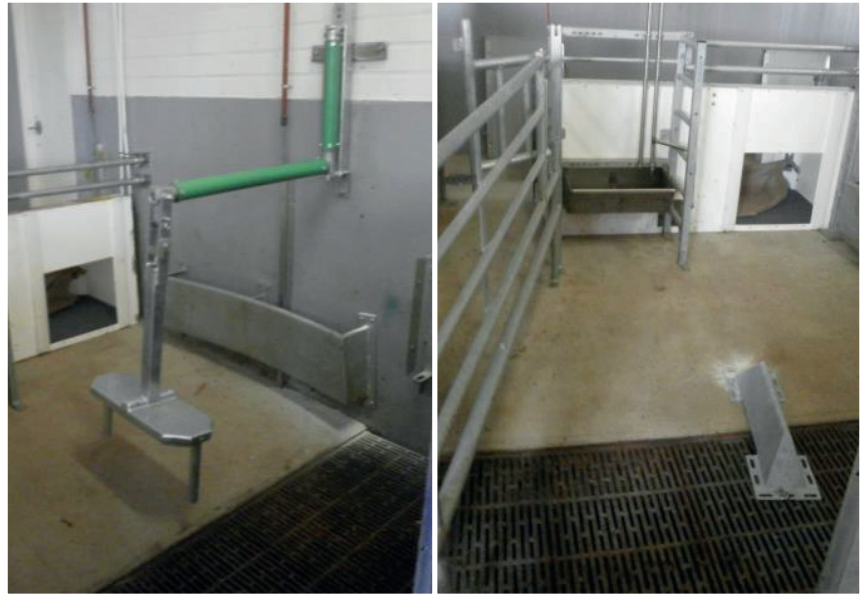

Figure 7.6. Devices added in the farrowing pens of the second version of the multi-suckling system, which aimed to limit piglet crushing. width of the farrowing pens was reduced (though sows were still able to turn around), to limit the sows' possibilities to lie down in the middle of the farrowing pen. Moreover, in one pen per unit, a 'swing' was installed (Figure 7.6, left photo) to slow down the movements of the sow in case she 
did lie down in the middle of the pen, giving piglets more opportunities to escape. In the remaining pens, metal bars were placed on the floor (Figure 7.6, right photo) to limit 'rolling over' behaviour, but the bars were later removed because they seemed to have a counterproductive effect.

Despite our attempts to limit piglet mortality, we observed higher pre-weaning mortality in the MS system than in the conventional system in Chapter 3. The majority (80\%) of pre-weaning mortality occurred before grouping of litters, and was mainly caused by crushing. Also in the second version of the system, overall live-born piglet mortality was high. On average 3.6 piglets died per litter before 4 weeks of age. As piglet mortality may reduce with increasing experience of stockpersons with the system ( $\mathrm{Li}$ et al., 2010), mortality may have remained high because different stockpersons were involved in the management of the first and second version of the system. Furthermore, the suboptimal possibilities to create separate microclimates for the sows and the piglets may have contributed to the risk of crushing. Room heating was available in the system but it was difficult to reduce ambient temperatures, and maximum temperatures were influenced by outside temperatures (mainly during summer). Sows and new-born piglets greatly differ in their thermal comfort zone (Black et al., 1993) and creating a temperature gradient between the piglet nest and the remainder of the farrowing pen can stimulate piglets to lie in the piglet nest, where they are protected from being crushed (Yajun et al., 2011). In the first system, this temperature gradient was, however, limited as average ambient temperatures $( \pm S D)$ were $21.6 \pm 1.8^{\circ} \mathrm{C}$ in the farrowing pens and $24.9 \pm 1.0^{\circ} \mathrm{C}$ in the piglet nests. Nonetheless, providing microclimates or other measures to improve the attractiveness of the piglet nest, may be limited in reducing piglet mortality, as new-born piglets are motivated to remain close to the sow (Vasdal et al., 2010).

Overall, the changes in management and farrowing pen design may have had little effect because piglet mortality in loose farrowing systems is largely affected by sow-related characteristics, rather than pen design (Weary et al., 1998; Andersen et al., 2005; Weber et al., 2009). Indeed, there was quite some variation between sows in the number of piglets that was crushed before grouping (ranging from 0 to 10 per litter in Chapter 3). This may be related to variability in maternal characteristics, such as the expression of piglet-directed pre-lying behaviour and responsiveness to piglet distress calls (Wechsler and 
Hegglin, 1997; Marchant et al., 2001). In addition, variation in the sows' physical condition may have had an effect, as e.g. poor leg condition or a large body size may result in uncontrolled lying down behaviour (Marchant and Broom, 1996; Marchant et al., 2001). Furthermore, piglet mortality may decrease if sows have previous experience with farrowing in a certain (alternative) farrowing system (Wechsler, 1996). The sows on our research farm, however, generally had no previous experience with loose farrowing. As the risk of crushing increases with litter size (Weary et al., 1998; Weber et al., 2009), the potential of the used sow genotype to produce large litters may also have contributed to high piglet mortality. There was only one sow genotype available on the research farm, which is selected for good performance in conventional systems. This type of sow may, however, be less suitable for alternative farrowing systems such as the MS system, which provide more freedom to express e.g. individual behavioural differences. In line with this, Baxter et al. (2011a) found an interaction between sow genotype and the farrowing environment, regarding piglet survival and maternal behaviour. This indicates the importance of a match between the characteristics of the sow genotype and the environment in which it has to produce.

\section{Piglet mortality in relation to group farrowing and lactation}

Besides risk factors related to (individual) loose farrowing, there are also risk factors for piglet mortality that are related to farrowing in groups, such as providing sows freedom to choose the site of farrowing. As indicated in Chapter 2; dispersed farrowing, farrowing in an occupied pen, and farrowing in the communal area can increase the risk of crushing, malnourishment, and hypothermia (Burgwal-Konertz, 1996). This was confirmed during the optimisation trials of the first system, in which sows occasionally farrowed in the communal area, with a suboptimal climate for the piglets, or in an occupied pen, increasing the risk of crushing. Therefore, it was decided to lock the sows in their own farrowing pen during the nights before farrowing. Moreover, during the day, a sow occasionally started the farrowing process in a different (albeit empty) pen than was assigned to her at entry in the system. This was likely related to the lack of a preference for a specific nest site at the time of entry in the system (i.e. 1 week before expected farrowing), as in nature, sows start looking for suitable nest sites 1 to 2 days before parturition (Jensen, 1986; Jensen et al., 1987; Stolba and Wood-Gush, 1989; Jensen et al., 
1993). In the first version of the system, a sow was moved to her assigned pen if she started the farrowing process elsewhere. As this may hamper the farrowing process (Lawrence et al., 1992), in the second version of the system it was decided to allow a sow to continue farrowing in the pen in which she started the parturition process.

In addition, in Chapter 2, increased piglet mortality after grouping of litters was identified as a risk factor, in comparison with systems with individually housed sows. It was suggested that mortality specifically due to crushing may be increased if MS housing is preceded by housing in farrowing crates because of exposure to 'dangerous' lying behaviour of the sows. In our system, however, sows did not experience a transition from confinement around farrowing to more freedom of movement in the multi-suckling phase. Nevertheless, overall post-grouping mortality tended to be higher in our system than in the conventional system $(0.54 \pm 0.07$ vs. $0.20 \pm 0.15$ piglets per litter, $P$ $=0.07$, Chapter 3 ). Perhaps this resulted from the possibility of sows with e.g. poor mothering ability to more easily trample or crush piglets from other litters as well after grouping, for instance in case sows and piglets crowded together around nursing in the communal area. On the other hand, sows with better mothering ability may have partly compensated; anecdotally, it was observed that during several crushing events, during which a sow did not respond to the squealing piglets, other sows rushed over and incited the sow to stand up.

As a recent study shows, however, an MS system does not necessarily result in overall higher levels of piglet mortality compared with conventional housing. Grimberg-Henrici et al. (2016a) even found overall lower pre-weaning piglet mortality in an MS system with farrowing crates than in conventional housing, and specifically, fewer piglets were crushed in the MS system (they did not specifically distinguish between mortality before and after grouping of litters). One of the reasons proposed by the authors for this difference is that stockpersons were experienced in managing the MS system, as the system had already been in use for 5 years.

\section{Piglet development in relation to mortality}

The measurements in this thesis focussed on the multi-suckling phase, the transition around weaning, and the period later in life, rather than the phase around farrowing (i.e. before grouping of litters). Nevertheless, the level of 
mortality may have affected the subsequent measurements. For instance, we cannot fully exclude the possibility that an enhanced performance of piglets raised in the MS system may result from a higher selection pressure for more vital piglets. Birth weight is a critical factor for piglet vitality and survival (Hoy et al., 1994; Tuchscherer et al., 2000; Baxter et al., 2008). Piglet vitality may be defined as the ability to quickly stand and become active directly after birth, locate the udder, compete with litter-mates for a teat, and drink colostrum (Edwards, 2002). In our study, piglets that survived had a higher birth weight than piglets that died, in both the MS system $(1.49 \pm 0.05$ vs. $1.33 \pm 0.03, P<$ $0.01)$ and the conventional system $(1.43 \pm 0.09$ vs. $1.00 \pm 0.06, P<0.01)$. Birth weight of piglets that died was, however, significantly higher in the MS system than in the conventional system $(P<0.05)$. This may be partly due to the tendency for a higher birth weight in the MS system (1.47 \pm 0.02 vs. $1.40 \pm$ $0.04 \mathrm{~kg} P<0.10$; this includes piglets born with the sows and piglets added by cross-fostering, and includes both piglets that survived and died before weaning). Nevertheless, this gives an indication that piglets with a lower birth weight did not die disproportionally more frequently in the MS system than in the conventional system and that selection for more vital piglets in the MS system may have been limited in comparison with the control system. The high levels of piglet mortality can, however, also affect litter size. Therefore, to reduce potential effects of a difference in litter size between treatments, litter sizes were equalised by cross-fostering within the first 48 hours after farrowing. This resulted in similar litter sizes at grouping in both housing systems (Chapter 3).

\subsection{Development of pigs in raised in the MS system}

Although some risk factors related to our MS system were identified, the overall expectation was that the MS system would promote piglet development, compared with conventional housing, and that a more gradual weaning process in the MS system would further enhance development, compared with abrupt weaning. In conventional pig farming, the weaning procedure consists of a dietary change -from a mainly milk-based diet to (solid) piglet feed- in combination with other abrupt changes that occur simultaneously, such as separation from the sow, relocation to a nursery, and mixing with unfamiliar piglets (see Figure 7.7). Mediated by stress and neophobia (i.e. an initial reluctance to eat novel food items (see Oostindjer et 
al., 2010)), this generally results in a poor post-weaning performance, characterised by a low feed intake, an impaired intestinal function, a growth check, diarrhoea, and behavioural indicators of distress, such as belly-nosing (Dybkjær, 1992; Vente-Spreeuwenberg and Beynen, 2003). In this section, it is discussed how the MS system can contribute to alleviating these issues, in terms of i) the pre-weaning environment in relation to preparation for the moment of weaning, ii) the transition to the post-weaning environment and iii) the more gradual weaning procedure during an extended lactation. Lastly, long-term consequences of different housing and weaning procedures are discussed, closing with the contribution of the MS system to the development of robust animals.

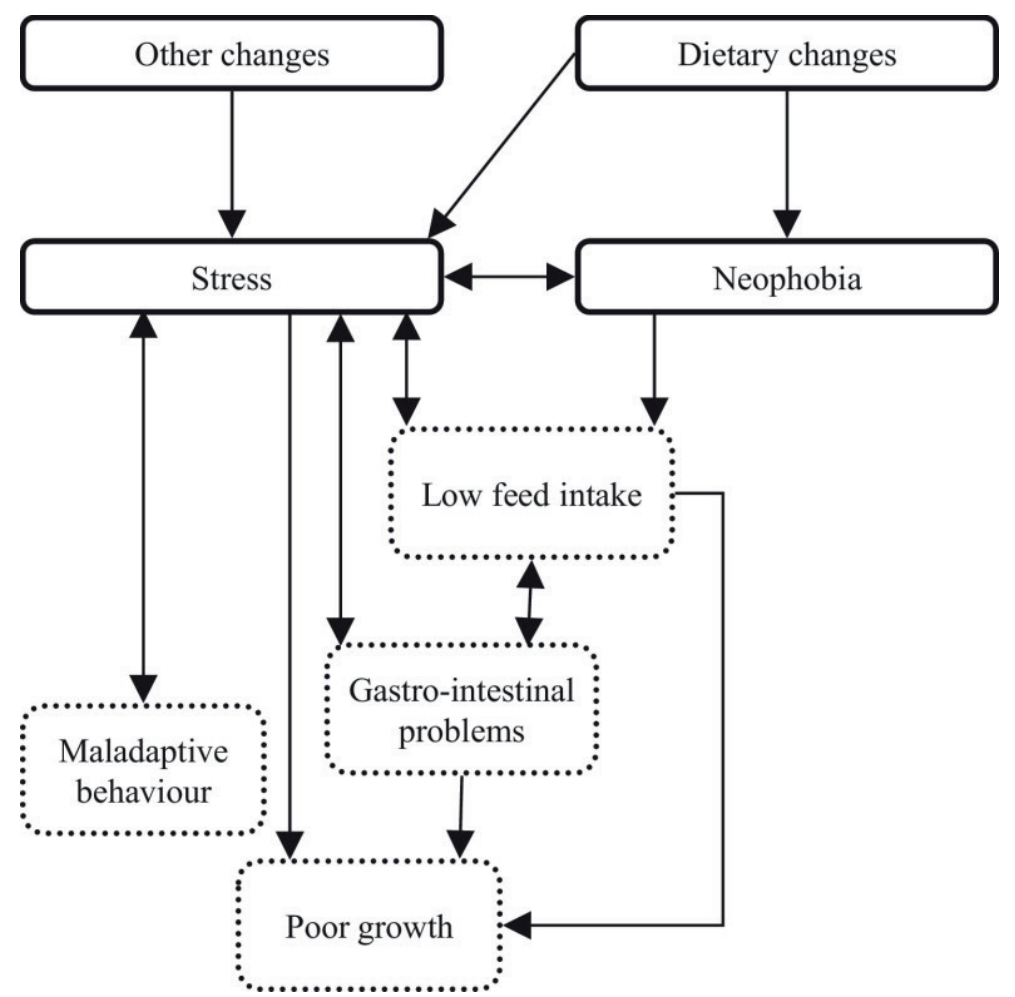

Figure 7.7. Overview of the relation between problems associated with weaning and their contributing factors, i.e. dietary and other changes that often occur at weaning in conventional pig farming, such as separation from the sow, relocation to a new environment, and mixing with unfamiliar piglets (Bolhuis et al., 2009). 
Before discussing the results, a short overview of the housing conditions and coherence of Chapters 3 to 6 is provided. Chapters 3 to 5 had similar contrasts in pre-weaning housing conditions (i.e. MS housing vs. conventional housing), and both treatment groups were weaned abruptly at 4 weeks of age (although piglets originated from the first version of the system in Chapter 3 and from the second version in Chapters 4 and 5). Contrasts in post-weaning housing, however, differed in Chapters 3 to 5. In Chapter 3, a similar level of postweaning social and physical enrichment was adopted as before weaning (i.e. MS piglets were continuously housed under enriched conditions and conventional piglets were housed continuously under barren conditions throughout the experiment). On the other hand, in Chapters 4 and 5, piglets from both pre-weaning housing treatments were housed post-weaning under physically enriched conditions. In Chapter 5, the same group of animals was studied as in Chapter 4. The MS animals used in Chapters 4 and 5 originated from two batches of the experiment run in Chapter 6. In Chapter 6, both treatment groups originated from the MS system, and treatment groups differed in weaning procedure.

\subsubsection{Pre-weaning environment and preparation for the moment of weaning}

In conventional systems, pre-weaning piglet feed intake is generally low, and varies greatly between individuals (Pajor et al., 1991; Bøe and Jensen, 1995; Bruininx et al., 2002). Consequently, piglets are generally not well-prepared for the dietary switch that occurs at weaning. In our system, pre-weaning experience with solid feed was stimulated by the communal feeding area, which facilitates social learning of foraging-related behaviours from the sows and other piglets by observation and participation (Morgan et al., 2001; Oostindjer et al., 2011). Additionally, the used environmental enrichment may stimulate behaviours related to foraging and exploration (Studnitz et al., 2007). Indeed, in Chapter 3, we found that piglets in the MS system more frequently showed feed-directed behaviour around 2 weeks of age than piglets in the conventional system. The MS piglets showed a considerable interest in the feed of the sows (overall, around $60 \%$ of the feed-directed behaviour was directed at the sow feed and $40 \%$ at the piglet feed). The frequency of feed-directed behaviour did not differ 2 days before weaning and actual pre-weaning intake of sow and piglet feed was not assessed. Nevertheless, the MS system seems 
promising to promote early experience with solid feed, which is important for early post-weaning feed intake and weight gain (Van den Brand et al., 2014). Likewise, in nature, piglets already display foraging-related behaviours, such as rooting, within a few days post-partum (Petersen, 1994) and start ingesting solids from 10 to 14 days of age (Gundlach, 1968; Meynhardt, 1980). This is around the time that the family group is joined, which provides opportunities to learn how to forage and what to eat in the social context of the family group.

Furthermore, to cope with changes in the social environment at weaning, preweaning development of social skills is important. In a conventional farrowing system, piglets lack physical contact with other sows and their litters, which can hamper social development (e.g. D'Eath, 2005). Furthermore, space and environmental enrichment are limited, while these aspects are important to stimulate the expression of a range of behaviours related to social development, such as play behaviour, (Spinka et al., 2001) and non-physical agonistic interactions, e.g. threatening and avoidance (McGlone and Curtis, 1985; Weng et al., 1998). Despite social contact with multiple sows and litters, and the provision of a more spacious and enriched environment in our MS system, we found that the frequency of aggressive behaviour (both total occurrence and occurrence of separate elements of aggressive behaviour) and the number of skin lesions related to aggression did not differ between housing systems before weaning in Chapter 3. Also the total occurrence of play, and of social play in particular, did not differ significantly (although, numerically, the occurrence of play behaviour was on average $44 \%$ higher in the MS system). It can be speculated that the contrast between both housing systems in the occurrence of social play specifically, was lower than expected due to a lack of space and suitable enrichment material in the conventional system. Possibly, this resulted in a direction of play behaviour towards conspecifics, rather than performing play individually or expressing substrate play.

Lastly, piglets may show maladaptive behaviour in response to weaning, such as damaging oral manipulation of conspecifics (Dybkjær, 1992). The development of such maladaptive behaviours can, however, already start before weaning (Ursinus et al., 2014). Damaging oral manipulation (e.g. tail or ear biting) is an expression of frustrated exploratory behaviour which is redirected towards conspecifics (Studnitz et al., 2007). This not only indicates impaired welfare of the actor, but can also inflict lesions on the bitten pigs, 
which can contribute to increased use of antibiotics (Schrøder-Petersen and Simonsen, 2001). Tail biting is a serious welfare and economical issue in conventional husbandry (e.g. Harley et al., 2014). Although the development of damaging behaviours is of multifactorial nature, provision of enrichment can contribute to its prevention (Studnitz et al., 2007; D'Eath et al., 2014). Indeed, before weaning we found that piglets showed less damaging oral manipulation in the more enriched MS system than in the conventional system in Chapter 3. Tail and ear lesions on the day before weaning were mostly limited to small bite marks and the damage scores did not differ between the 2 systems. Nevertheless, early prevention of manipulative behaviours seems important to prevent tail biting later in life (Telkänranta et al., 2014). Currently, tail docking is widely used as a measure to prevent tail biting and related issues (Nannoni et al., 2014), despite the prohibition of routine use of this method in the EU (Council Directive 2008/120/EC). As the research farm where our studies were conducted experienced issues with tail biting, piglets were also tail docked in our experiments. Alternatives to this measure are necessary, however, as the procedure itself impairs welfare and is not fully effective to prevent damaging behaviours (Nannoni et al., 2014) and there is increasing societal pressure to comply with EU legislation.

\subsubsection{Early post-weaning performance after abrupt weaning at 4 weeks of age in relation to (the transition to) the post-weaning environment}

In line with the higher frequency of pre-weaning feed-directed behaviour observed in the MS system; in Chapter 3, we found that the piglets raised in the MS system seemed to have less difficulty making the transition from a milkbased diet to a fully solid diet at weaning than the piglets raised in the conventional system. This was indicated by a higher feed intake (days 0 to 13 : $4.69 \pm 0.36$ vs. $3.46 \pm 0.38 \mathrm{~kg}$ per piglet, $P<0.10$ ), a higher weight gain (days 1 to $13: 3.81 \pm 0.37$ vs. $2.58 \pm 0.23 \mathrm{~kg}$ per piglet, $P<0.05$ ) and more solid faecal consistency scores, indicating a lower occurrence of diarrhoea, in the first two weeks after weaning. Correspondingly, we observed a lower peak in belly nosing behaviour. The expression of belly-nosing may result from frustrated suckling behaviour, and the lower frequency may reflect a more advanced level of ingestive development in piglets raised in the MS system (reviewed by Widowski et al., 2008). The higher post-weaning feed intake may be related to the earlier onset of pre-weaning sampling of solid feed (see Van 
den Brand et al., 2014) and social facilitation of eating behaviour in the larger group in which MS-raised piglets were housed post-weaning (Morgan et al., 2001; Figueroa et al., 2013). Possibly, social facilitation of post-weaning eating behaviour may also have been related to the adoption of a different foraging strategy, making more use of social information, compared with the conventionally reared piglets, as suggested in Chapter 5. Moreover, Chapter 4 gives some indication that the observed differences in post-weaning performance in Chapter 3 may partly be explained by a difference in intestinal function, reflected in a difference in early post-weaning feed utilisation and carbohydrate absorption. In Chapter 4, piglets reared in the MS system had lower plasma concentrations of mannitol (320 \pm 116 vs. $592 \pm 120 \mathrm{nmol} / \mathrm{mL}$, $P=0.04)$ and galactose $(91 \pm 18$, vs. $157 \pm 19 \mathrm{nmol} / \mathrm{mL}, P=0.04)$ than conventionally reared piglets, 20 minutes after oral administration on day 5 post-weaning. In addition, piglets reared in the MS system seemed to have a better feed utilisation than conventionally reared piglets in the early postweaning period, as piglets reared in the MS system gained more weight than conventionally reared piglets between days 2 and 5 post-weaning $(0.67 \pm 0.12$ kg vs. $0.39 \pm 0.16 \mathrm{~kg}, P=0.02$ ), despite a similar feed intake. Differences in intestinal function may be mediated by differences in pre-weaning experience with solid feed and/or differences in experiencing stress (see Figure 7.7 and e.g. Moeser et al. 2007).

The results of the performed sugar absorption test were, however, not straightforward to interpret. In order to better comment on intestinal permeability for instance, simultaneous administration of two carbohydrates differing in size may have provided more insight, as the ratio of the urinary recovery of the two sugars provides information about intestinal barrier function. By using a ratio, factors such as intestinal transit time can be accounted for, and urinary collection may provide a more accurate permeability estimate than a single blood sample (Wijtten et al., 2011). Additionally, supporting measures of intestinal morphology would have provided more insight into intestinal function around weaning (VenteSpreeuwenberg and Beynen, 2003). Piglets could, however, not be sacrificed because all were needed for the subsequent study of Chapter 5 .

Furthermore, piglets raised in the MS system may have coped better with the change in social environment after weaning as they showed fewer aggression- 
related skin lesions on days 2 and 5 post-weaning than conventionally reared piglets (Chapter 3). Although neither of the treatment groups was mixed with unfamiliar animals at weaning, group size was altered due to selection of animals for further post-weaning investigation. This may have been accompanied by a rearrangement of dominance ranks. Compared with singlelitter housing, pre-weaning contact with multiple litters has been shown to reduce aggression after weaning towards familiar pigs (Hessel et al., 2006) and to stimulate quicker formation of a stable dominance hierarchy after mixing with unfamiliar animals post-weaning (D'Eath, 2005). Interestingly, despite the difference in skin lesions, we did not observe a difference in the post-weaning frequency of aggressive behaviours between the treatment groups. Similarly, in Chapter 5, piglets raised in the MS system or in the conventional system showed a similar frequency of aggressive behaviours during a feed competition test, which was performed with pairs of familiar animals. During the test, 1 relatively light and 1 relatively heavy piglet competed for access to a bucket with feed. Unexpectedly, we found indications that the conventionally reared piglets established more clear dominance relationships during the feed competition test than the piglets reared in the MS system, which is in contrast with findings of (De Jonge et al., 1996). For instance, in Chapter 5, the difference in number of access bouts to the bucket with feed was larger within the conventionally reared pairs than within the MS-reared pairs. We suggested that this finding may be related to a difference in the context of obtaining feed in both pre-weaning farrowing systems; the MS piglets were stimulated to eat together in the communal feeding area, whereas the conventional piglets had only one small piglet feeder for the whole litter and therefore had a more competitive environment to obtain solid feed. To come back to the lower number of skin lesions observed in the MS-reared piglets in Chapter 3; this may be a more general reflection of less frustration-associated aggression (see Dantzer et al., 1980), related to an overall better transition to the situation after weaning, which is not necessarily related to the change in social environment. For instance, piglets reared in an MS system may cope better with a change in physical environment than piglets reared with an individually housed sow (Hillmann et al., 2003).

Altogether, the interplay between stress, behaviour, feed intake, weight gain, and intestinal function (Figure 7.7) makes it impossible to distinguish cause and effect regarding our observations on post-weaning performance. In 
addition, the relative contribution of pre-weaning and post-weaning conditions is difficult to distinguish, as a piglet's post-weaning performance depends on an interaction between its pre-weaning development, post-weaning housing conditions, and the relative appreciation of the change in environment. As experiencing a reduction in the level of environmental enrichment may be detrimental (Bolhuis et al., 2006; Munsterhjelm et al., 2009), we provided piglets from both the MS system and the conventional system a transition to a similar or increased level of environmental enrichment. A challenge is, however, that it is not possible to provide an 'equal' post-weaning environment for both pre-weaning treatment groups. It is an option to i) provide a postweaning environment which is similar to the pre-weaning environment, thus further extending the system comparison into the post-weaning phase, making it impossible to separate pre-weaning and post-weaning factors (as was done in Chapter 3), or ii) provide the same post-weaning housing conditions for both treatment groups, thus creating a different relative transition from the preweaning to the post-weaning environment, which can, in turn, affect the piglets' performance (as was done in Chapters 4 and 5). The post-weaning housing conditions may especially impact piglet performance around the transition phase. To illustrate; in Chapter 4, no overall differences were found between weaning and two weeks thereafter, in feed intake, weight gain, and faecal consistency scores between piglets raised in the MS system or in the conventional system. This is in contrast with the findings of Chapter 3. To illustrate these differences, Figure 7.8 shows feed intake and weight gain in both experiments. The figure suggests that, for the conventionally reared piglets, the enriched post-weaning housing conditions may have partly compensated for the relatively barren pre-weaning housing conditions, considering the cumulative feed intake over the first 2 weeks post-weaning. In terms of total weight gain in the first 2 weeks after weaning, the reduced contrast between both treatment groups in Chapter 4 vs. Chapter 3 is more apparent for both groups; piglets raised in the MS system seemed to perform slightly worse in Chapter 4 than in Chapter 3, whereas piglets raised in the conventional system seemed to perform slightly better in Chapter 4 than in Chapter 3. Nonetheless, such comparisons of results from different experiments remain speculative. 

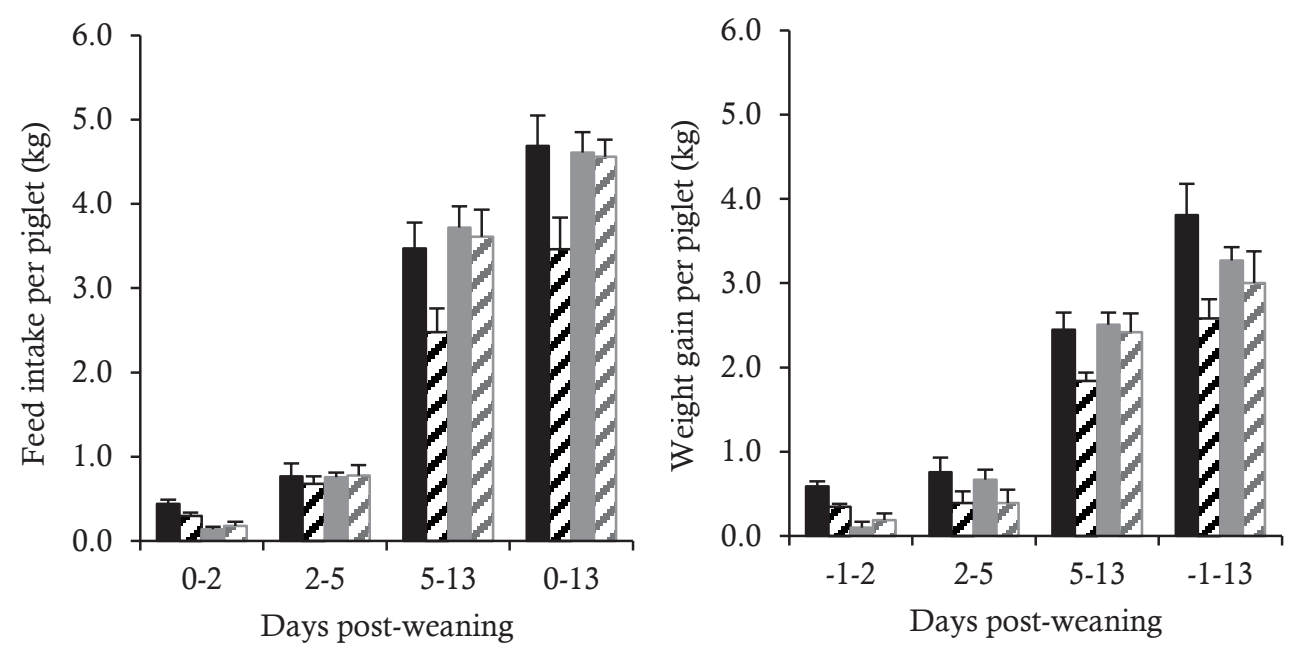

Figure 7.8. Post-weaning piglet feed intake and body weight gain in Chapter 3 (black bars) and Chapter 4 (grey bars). Solid bars represent piglets raised pre-weaning in the MS system. Dashed bars represent piglets raised pre-weaning in a conventional system. No statistical significance is included, as inter-experimental differences were not analysed.

\subsubsection{A more gradual weaning process during an extended lactation}

The issues related to weaning, as depicted in Figure 7.7, are not only related to pre- and post-weaning housing conditions, but also to the timing and the abruptness of the weaning procedure itself. In a conventional system, weaning occurs at a relatively young age (i.e. 3 to 4 weeks). At this time, piglets are still immature in terms of the development of e.g. their immune system and gastrointestinal tract (King et al., 2003; Miller and Slade, 2003). Moreover, separation from the sow occurs abruptly, while piglets still rely on milk as their main source of nutrients (Weary et al., 2008). In contrast, weaning is a gradual process in nature, which starts already in early lactation (Jensen et al., 1991) and is not completed until 2 to 5 months of age (Meynhardt, 1980; Newberry and Wood-Gush, 1985; Jensen, 1986; Jensen and Recén, 1989). The weaning process is characterised by a gradual decrease in nursing frequency (Jensen, 1988; Jensen and Recén, 1989; Jensen and Stangel, 1992), and an increase in intake of solids by the piglets (Jensen, 1995). In our system, a more gradual weaning process was not only stimulated by providing gradual transitions in housing from the pre-weaning to the post-weaning phase, and providing more stimulating environments for piglet development, but also by changing the management of the weaning procedure itself. In Chapter 6, a gradual increase 
in separation between sows and piglets was enabled by a combination of forced intermittent suckling (IS) in the fifth week of lactation, followed by voluntary use of the IS area by the sows during a lactation period of 9 weeks. We expected that this would further enhance development (e.g. stimulate independence) of pigs raised in the MS system, compared with more abrupt and earlier weaning.

Forced IS can contribute to a more gradual transition to the post-weaning period, by reducing nursing frequency and stimulating piglets' pre-weaning experience with solid feed (Kuller et al., 2004; Berkeveld et al., 2007a; Kuller et al., 2007; Kuller et al., 2010). Indeed, we found that nursing frequency was significantly lower during forced IS than in the preceding week (17.6 \pm 1.1 vs. $26.4 \pm 1.6$ per 24 hours, albeit we did not have an MS group without forced IS as a control group in this timeframe). During the period in which sows could voluntarily separate from the piglets (weeks 6 to 9), nursing frequency also gradually declined, with a significantly lower frequency on day $56(10.1 \pm 2.6)$ than on day $35(21.2 \pm 2.3)$. The cumulative percentage of sows that had no successful nursing bouts was $8.3 \%$ on day $42,12.5 \%$ on day 49 and $20.8 \%$ on day 56. The range in frequency of successful nursing bouts (i.e. with milk letdown) was 0 to 22 on day 56. The sows' use of the piglet-free IS area increased (see Figure 7.9) between days 35 and 56 of lactation $(P<0.01)$, and the percentage of time spent in the IS area was negatively correlated with the number of successful nursing bouts per 24-hour period ( $\mathrm{r}=-0.74, P<0.0001$ ). The variation in nursing frequency and use of the IS area between sows indicates a variation in weaning process of the piglets (also see Pitts et al., 2002). A potential risk within an MS system is that piglets which are weaned by their mother try to cross-suckle with the sow(s) that are still lactating. The number of non-offspring piglets present at the udder (mean \pm SD) per successful nursing bout did show an increase over time (day 21: $2.07 \pm 1.85$, day 35: $2.42 \pm 1.99$, day 42: $2.36 \pm 1.98$, day 49: $2.78 \pm 2.17$, day 56: $3.42 \pm$ 2.17 , including cross-massagers and cross-sucklers), although these data were not statistically analysed. The consequences of varying weaning processes for individual piglet performance could, however, not be determined, as e.g. suckling frequency and feed intake were not registered individually.

We not only found variation in nursing frequency and the use of the IS area between sows, but also between batches. For instance, the use of the IS area in 
the second batch in Chapter 6, was considerably higher than in the other 4 batches (Figure 7.9). Correspondingly, nursing occurred less often than in the other batches, with nursing frequencies close to zero from day 42 onwards in batch 2. It can be speculated that this is because the IS area consisted of a covered outdoor area, which may be more attractive for the sows with increasing indoor temperatures in summer (weeks 4 to 9 of lactation occurred in summer only in batch 2). Possibly, a contrast in temperature between the IS area and the area with the piglets can be beneficial to further stimulate a gradual weaning process. Thus, besides reducing contact with the piglets, the use of the IS area by the sows may have other motivations, such as a more comfortable climate.

No other studies were found that combined an MS system with forced IS and a voluntary separation by the sows in late lactation. However, studies in which forced IS was combined with an extended lactation showed that piglets displayed more eating behaviour before weaning (Berkeveld et al., 2007a) and showed an improved transition to the post-weaning phase, indicated by a higher feed intake, reduced growth check, improved intestinal function, and reduced aggression and damaging oral manipulation (Berkeveld et al., 2007a; Berkeveld et al., 2007b; Berkeveld et al., 2009; Turpin et al., 2016), compared with conventionally weaned piglets. Similarly, studies providing a piglet-free area for the sows found a higher pre-weaning feed intake, a higher post-

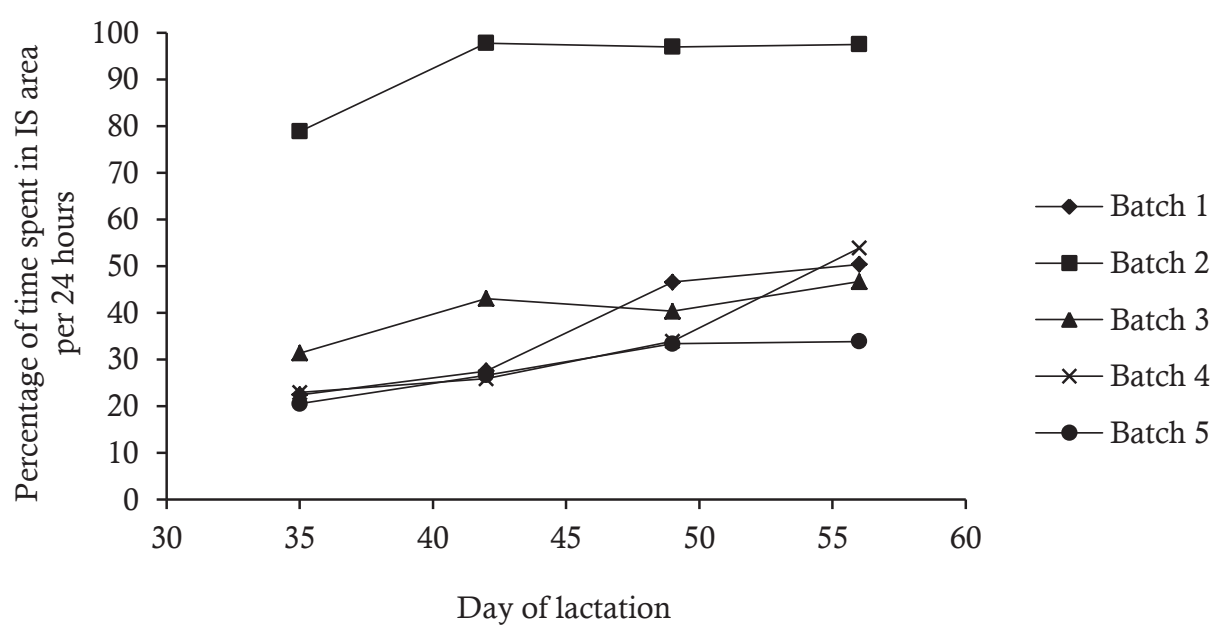

Figure 7.9. Average percentage of time spent by the sows in the intermittent-suckling area per batch. 
weaning feed intake, a higher post-weaning weight gain (Weary et al., 2002), and more post-weaning eating behaviour (Rantzer et al., 1995) than in piglets reared with individually housed sows. Additionally, extending the age at weaning, without the use of IS or a piglet-free area, has been reported to reduce post-weaning belly-nosing behaviours (Bøe, 1993). We found that abrupt weaning at 4 weeks of age seemed to have more impact than 1 week of forced IS, followed by voluntary separation by the sows. Within the first 2 weeks after starting the different weaning procedures, abruptly weaned piglets showed a reduction in average daily gain and a distinct peak in belly-nosing behaviour, which were not shown by the piglets remaining in the MS system. This may e.g. reflect difficulties coping with the transition from a milk-based diet to a diet of only solid feed in the weaned piglets (see Widowski et al., 2008). In addition, the percentage of pigs scored as having pasty or watery faeces was twice as high in the group of abruptly weaned pigs, indicating a numerically higher occurrence of diarrhoea compared with the group that was subjected to the gradual weaning treatment. Contrastingly, for the piglets weaned at 9 weeks of age, weaning did not result in a growth check or any behavioural indicators of having difficulty coping with the post-weaning situation. Thus, the piglets that were subjected to the gradual weaning treatment coped better with both transitions around 4 and 9 weeks of age.

\subsubsection{Long-term effects of housing and weaning procedure}

We did not only find differences between treatment groups before weaning and around transition periods, but we also found more long-term effects during the nursery and finishing phases. In Chapter 3 , the piglets reared in the MS system showed a higher weight gain over the entire nursery phase between 4 and 9 weeks of age compared with piglets reared in the conventional system, resulting in a $3.4 \mathrm{~kg}$ heavier body weight at 9 weeks of age. In the subsequent finishing phase, during which pigs from both treatment groups were housed under conventional conditions after finishing the experiment, MS-reared pigs maintained their higher body weight, resulting in a higher weight at slaughter (94.7 kg vs. $93.0 \mathrm{~kg}, \mathrm{P}<0.05$, also see Van der Peet-Schwering et al., 2015). There were no differences between treatment groups in average daily gain, average daily feed intake, and feed conversion during the finishing phase. As experiencing a reduction in the level of environmental enrichment may be detrimental (Bolhuis et al., 2006; Munsterhjelm et al., 2009), it was remarkable 
that the MS pigs maintained their higher body weight, even after a transition to conventional housing which was preceded by more enriched conditions until 9 weeks of age.

Furthermore, we found long-term differences in behaviour in Chapter 3; piglets reared in the MS system showed a higher frequency of play behaviour and a lower frequency of damaging oral manipulation than the conventionally reared piglets over the entire nursery phase. Similar to the situation before weaning, oral manipulation of pen mates may have been limited by the availability of rooting and chewing materials after weaning (Bolhuis et al., 2005), whereas the larger total area and availability of enrichment materials may have stimulated play behaviour (Chaloupková et al., 2007). Moreover, these behavioural differences may reflect the improved transition to the post-weaning phase of the piglets raised in the MS system. In addition, in Chapter 5 we found indications for long-term differences in foraging strategies between piglets reared in the MS system and in the conventional system, during an 'informed forager test' (based on Held et al., 2000). Differences were found up until 11 weeks post-weaning, despite equal post-weaning housing conditions of both treatment groups. In the last phase of the test, dominant pigs (which were unaware of the location of a food reward in a testing arena with hidden buckets) were allowed to access the arena together with a submissive sibling (which was informed about the location of the food reward). We found indications that pigs raised in the MS system made more use of social information from their partner than pigs raised in the conventional system. For instance, among MS-reared pairs, the percentage of testing trials in which the 'non-informed' (dominant) pig ate from the baited bucket was positively correlated with the number of times the non-informed pig displaced its 'informed' (submissive) partner from a bucket $(r=0.84, P=0.01)$. On the other hand, among conventionally raised pairs, the percentage of trials in which a non-informed pig ate from the baited bucket was positively correlated with the mean number of buckets the non-informed pig visited itself per testing trial ( $\mathrm{r}=0.77, P=0.03$ ). Possibly, the suggested differences in foraging strategy arose from a difference in the context of obtaining feed before weaning (i.e. in the communal feeding area in the MS system and from a separate and relatively small piglet feeder in the conventional system). Moreover, we speculated that a different predisposition for a certain coping style during rearing in either the MS or the conventional system may be related to 
differences in foraging strategies. The use of social information is, for instance, related to a reactive coping style rather than to a pro-active coping style (Benus et al., 1990; Kurvers et al., 2010a; Kurvers et al., 2010b). Lastly, as a side note, it is interesting to remark that differences in aspects of intestinal function in Chapter 3 may possibly be related to differences in behaviour shown by these same animals in Chapter 5, as several studies have shown that behaviour can be modulated via the bidirectional microbiota-gut-brain axis in mammals (Cryan and O'Mahony, 2011; Heijtz et al., 2011; Collins et al., 2012).

In the 'informed forager test' we also tested aspects of non-social cognitive performance, during a series of trials in which pigs were tested individually. We expected that piglets raised in the MS system would perform better than conventionally raised piglets, due to the more complex social and physical rearing environment of the MS system. In Chapter 5, however, we found that in the process of training the 'informed' piglets to learn their task, piglets raised in the MS system and in the conventional system needed a similar number of testing trials to reach the 'learning criterion'. Also, the number of revisits to buckets that were already previously investigated in the testing arena (considered as a measure of impaired working memory (van der Staay et al., 2012)) did not differ between treatment groups. This is in contrast with studies in which pigs housed under enriched conditions demonstrated a better short term and/or long-term memory than pigs housed under barren conditions (de Jong et al., 2000; Bolhuis et al., 2013; Grimberg-Henrici et al., 2016b). In these studies, pigs were, however, housed in contrasting environments at the time of the cognitive tests, whereas in our study pigs were housed under the same conditions already for 4 weeks at the start of testing and during the subsequent 2 weeks of testing. Possibly, the timing and duration of exposure to the contrasting pre-weaning environments up till 4 weeks of age may have limited potential effects of pre-weaning housing conditions on the measured aspects of cognitive performance, as the period of most rapid brain growth occurs until about 6 weeks of age (Dickerson and Dobbing, 1967), which may be the most sensitive period for environmental conditions to affect aspects of cognitive development.

Lastly, in Chapter 6 we found several long-term beneficial effects of the gradual weaning treatment within the MS system on behaviour during the finishing phase, although differences between treatment groups in production 
performance disappeared in the period after 9 weeks of age. For instance, during the finishing phase belly-nosing persisted at numerically higher levels in pigs weaned at 4 weeks of age, whereas pigs weaned at 9 weeks of age performed almost no belly-nosing. Similarly, Gonyou et al. (1998) found longterm effects of weaning age on the occurrence of belly-nosing. Additionally, in the tenth week of the finishing phase, pigs subjected to the more gradual weaning procedure had less skin damage related to aggression and oral manipulation. The latter was supported by lower levels of damaging manipulative behaviour from 4 weeks of age up until the finishing phase compared with pigs weaned abruptly at a younger age $(1.2 \pm 0.2$ vs. $1.8 \pm 0.2$ occurrences per hour, $P<0.05$ ). This may indicate less frustration (SchrøderPetersen and Simonsen, 2001) and may reflect the smoother transitions around weeks 4 and 9 for the pigs subjected to the gradual weaning treatment.

\subsubsection{The multi-suckling system in relation to animal robustness}

In a broader perspective, the MS system fits with the necessity to develop more robust animals, which contributes to sustainable animal production (de Goede et al., 2013). The robustness of an animal refers to its relative vulnerability to disturbance, and this vulnerability has three aspects i) exposure to disturbance, ii) resistance to disturbance, and iii) resilience to recover after a disturbance (de Goede et al., 2013). The MS system contributes to robustness by limiting external exposure to disturbances, e.g. avoiding relocation to a nursery, and by enhancing internal resistance and resilience, e.g. stimulating physical and behavioural development by providing a more suitable environment and management of more gradual transitions compared with a conventional system.

Weaning can be considered as a major challenge to the robustness of an animal. Our results indicate that the piglets reared in the MS system are better able to cope with this challenge than conventionally reared pigs, and that enabling a more gradual weaning process during an extended lactation can further improve piglets' resistance and resilience to weaning. This was reflected in a variety of measurements, i.e. behaviour, physical lesions, production performance, and measures related to intestinal function. As piglets reared in the MS system were housed under more enriched post-weaning housing conditions than the conventionally raised piglets, however, the weaning 
challenge was not studied under completely comparable conditions. It can be questioned whether the provision of an enriched post-weaning environment is necessary to maintain the enhanced performance of the MS pigs, or that early life conditions resulted in the development of piglets that are robust enough to withstand the weaning challenge under more adverse conditions as well. Moreover, it would be interesting to compare aspects of resistance and resilience in the MS system and conventional housing, by providing other environmental challenges during rearing and later in life. For instance, van Dixhoorn et al. (2016) found that piglets housed under physically and socially enriched conditions were less susceptible to disease than conventionally housed pigs, after a combination of viral and bacterial exposure.

Lastly, as piglets raised pre-weaning in the conventional system were not mixed with unfamiliar animals at weaning in any of our studies, and were housed under enriched post-weaning conditions in Chapters 4 and 5, more beneficial effects of the MS system on piglet development and robustness may be expected in comparison with a control group that would be kept under more commercial conditions. The expected contrast in piglet performance may be even larger if such a control group would be compared with the MS system in which a more gradual weaning procedure is enabled.

\subsection{Future optimisation and practical implementation}

To close, some aspects that can still be improved in the MS system are discussed, as well as some success factors that can be implemented in conventional husbandry.

\subsubsection{Future optimisation of the MS system}

Piglet mortality remains an issue in our MS system, in relation to welfare, but also in relation to commercial implementation of the system. Temporary crating of the sows around farrowing (i.e. from day 114 of gestation to day 4 after farrowing) may reduce total pre-weaning mortality compared with continuous loose housing (Hales et al., 2015). This is, however, not in line with the major starting point of our system, which is to enable the expression of natural behaviour. Moreover, several large-scale studies showed that commercial farms with continuous loose housing of sows around farrowing and lactation can achieve pre-weaning survival similar to conventional farms 
(Weber et al., 2007; Kilbride et al., 2012). Thus, an alternative to temporary crating is to use farrowing pens in the MS system which have been proven to achieve acceptable levels of piglet mortality under commercial conditions, e.g. 'PigSAFE' pens (Edwards et al., 2011). This option has been considered during the optimisation trials, but was not adopted at the time due to costs and the questionable necessity of some pen features which are important when sows are kept in their farrowing pen during the entire lactation period, but may be less relevant in combination with an MS system (e.g. more space allowance and the use of a separate feeding area and dunging area within the farrowing pens). Adjusting farrowing pen design can, however, negatively impact performance of the system (Baxter and Edwards, 2010). Possibly, a more promising strategy to reduce piglet mortality in the MS system may be the use of sows with more suitable, and less variable, characteristics related to e.g. mothering ability (see section 7.3.). In addition, using sows with experience in loose farrowing systems may limit piglet mortality (see Wechsler, 1996).

In relation to piglet development, the compromise in the use of enrichment materials may not have been optimal. For instance, enrichment could have been provided in the communal feeding area as an extra stimulation of foraging-related behaviours of the piglets (Oostindjer et al., 2014). Also, the use of bedding material could have contributed to alleviating the persisting problems with lameness in the piglets (Zoric et al., 2008) and could have better stimulated the sows' and piglets' behavioural need to root, as compared with the used concrete and metal flooring. In relation to the weaning procedure, a more gradual transition could have been achieved by leaving the piglets in the farrowing unit for one or several days after the sows were removed from the system. This would separate the moment of the dietary switch and complete absence of the sows from the moment of relocation to a new environment. Hence, the number of concurrent stressors around weaning could have been further reduced.

Furthermore, we only used multiparous sows in our experiments (ranging from parity 2 to 6), because primiparous animals may have a poorer performance. Therefore, experience with primiparous sows is important in relation to commercial adoption of the MS system. Compared with multiparous animals, primiparous animals may show more savaging behaviour of their new-born piglets (Harris et al., 2003; Chen et al., 2008), and specifically in an MS system, 
they may be more susceptible to teat damage related to cross-suckling (Bohnenkamp et al., 2013), and to aggression-related skin lesions due to a lower dominance rank (Hultén et al., 1995b). Moreover, induction of lactational oestrus and ovulation may be less effective in primiparous animals (Hultén et al., 1995a; Hultén et al., 2006; Soede et al., 2012).

Lastly, for a successful system performance, not only animal performance is important, but also aspects such as environmental impact and economical feasibility should be considered. These aspects were beyond the scope of this thesis, but nevertheless require further research in relation to commercial implementation of the MS system.

\subsubsection{Implementation of aspects of our MS system in conventional husbandry}

On another note, there are some aspects of the MS system that can be implemented in conventional farrowing systems. For instance, sows can be given more freedom of movement during lactation by only crating them when the risk for piglet mortality is highest, i.e. during the first 3 days post-partum (Wechsler and Weber, 2007). In addition, manipulable nest building material and enrichment material, which is compatible with conventional slurry management, can be provided for both sows and piglets around farrowing and lactation. Environmental enrichment in combination with freedom of movement can positively affect sow welfare, maternal behaviour, and piglet development, e.g. by limiting issues with the development of damaging oral behaviours in early life (Barnett et al., 2001; Studnitz et al., 2007; Yun and Valros, 2015). Moreover, the use of suitable enrichment materials can be extended to conventional nurseries and finishing units, to alleviate stress around weaning and further limit frustration and redirection of exploratory behaviour (Schrøder-Petersen and Simonsen, 2001; Studnitz et al., 2007). Furthermore, pre-weaning mingling of litters, to stimulate piglets' social development and prevent mixing with unfamiliar animals at weaning, may be incorporated in conventional husbandry by removing part of the pen partitions of adjacent farrowing pens (D'Eath, 2005; Kutzer et al., 2009). In addition, piglets' experience with piglet feed before weaning in conventional husbandry can be stimulated by making the sow feeder more accessible to the piglets, and providing piglet feeders with more space for the litter to eat simultaneously, 
which are positioned close to the sow feeder. Hence, observation, participation, and social facilitation of eating behaviour are stimulated, which may provide a more gradual transition to the post-weaning diet (Morgan et al., 2001; Oostindjer et al., 2011). Lastly, extending the weaning age may not be feasible, as conventional pens may not be suitable to accommodate older piglets due to limitations in pen size. In addition, the use of intermittentsuckling may not be feasible in current conventional systems, as the procedure may need automation to reduce labour requirements and there may be no designated place to relocate the sows during IS.

\subsection{Conclusions}

To conclude, our results support the expectation that the MS system would promote piglet development, compared with conventional housing, and that a more gradual weaning procedure during an extended lactation in the MS system would further enhance piglet development, compared with abrupt weaning at 4 weeks of age. We found differences in performance before weaning, around weaning-related transition periods, and later in life, which were reflected in behaviour, physical lesions, production performance, and measures related to intestinal function. Specifically, the lower occurrence of maladaptive behaviours (such as belly-nosing and tail biting) in the MS system compared with conventional housing, suggests that the MS system, especially in combination with a more gradual weaning procedure, fits with the development of systems in which harmful procedures such as tail docking can be abolished (also see de Goede et al., 2013). Pre-weaning piglet mortality was, however, an issue in the MS system, which was likely the result of a suboptimal farrowing environment in combination with the use of a type of sow that may perform well in a conventional system but may be less suitable for alternative farrowing systems such as the MS system. The lack of experience of the used sows with loose farrowing may also have played a role in this.

In general, enabling gradual transitions throughout life, via housing and management, seem important for sow and piglet performance. Specifically in relation to alleviation of weaning-related issues encountered in conventional systems, the MS system can contribute by i) better pre-weaning preparation of the piglets for the moment of weaning by providing a more stimulating social 
and physical environment, ii) eliminating or reducing the number of concurrent stressors at weaning, and iii) enabling a more gradual weaning procedure during an extended lactation. Post-weaning performance may be further enhanced by providing a physically and socially enriched post-weaning environment. 


\subsection{References}

Andersen I.L., Berg S., and Bøe K.E. 2005. Crushing of piglets by the mother sow (Sus scrofa) - purely accidental or a poor mother? Applied Animal Behaviour Science 93: 229-243.

Arey D.S. and Edwards S.A. 1998. Factors influencing aggression between sows after mixing and the consequences for welfare and production. Livestock Production Science 56: 61-70.

Aubel E., Baumgartner J., Bussemas R., Früh B., Hagmüller W., and Simantke C. 2011. Gruppensäugen in der Bioschweinehaltung. Forschungsinstitut für biologischen Landbau (FiBL).

Barnett J.L., Hemsworth P.H., Cronin G.M., Jongman E.C., and Hutson G.D. 2001. A review of the welfare issues for sows and piglets in relation to housing. Australian Journal of Agricultural Research 52: 1-28.

Baxter E.M. and Edwards S.A. 2010. Recommended dimensions and details for building PigSAFE pens. http://www.ncl.ac.uk/media/wwwnclacuk/agriculturefoodandruraldevelop ment/files/Recommended\%2520design\%2520criteria\%2520for\%2520building \%2520PigSAFE $\% 2520$ pens $\% 2520-\% 25$.pdf.

Baxter E.M., Jarvis S., D’Eath R.B., Ross D.W., Robson S.K., Farish M., Nevison I.M., Lawrence A.B., and Edwards S.A. 2008. Investigating the behavioural and physiological indicators of neonatal survival in pigs. Theriogenology 69: 773-783.

Baxter E.M., Jarvis S., Sherwood L., Farish M., Roehe R., Lawrence A.B., and Edwards S.A. 2011a. Genetic and environmental effects on piglet survival and maternal behaviour of the farrowing sow. Applied Animal Behaviour Science 130: 28-41.

Baxter E.M., Lawrence A.B., and Edwards S.A. 2011b. Alternative farrowing systems: Design criteria for farrowing systems based on the biological needs of sows and piglets. Animal 5: 580-600.

Benus R., Den Daas S., Koolhaas J., and Van Oortmerssen G. 1990. Routine formation and flexibility in social and non-social behaviour of aggressive and non-aggressive male mice. Behaviour 112: 176-193. 
Berkeveld M., Langendijk P., Bolhuis J.E., Koets A.P., Verheijden J.H.M., and Taverne M.A.M. 2007a. Intermittent suckling during an extended lactation period: Effects on piglet behavior. Journal of Animal Science 85: 3415-3424.

Berkeveld M., Langendijk P., Soede N.M., Kemp B., Taverne M.A.M., Verheijden J.H.M., Kuijken N., and Koets A.P. 2009. Improving adaptation to weaning: Effect of intermittent suckling regimens on piglet feed intake, growth, and gut characteristics. Journal of Animal Science 87: 3156-3166.

Berkeveld M., Langendijk P., Van Beers-Schreurs H.M.G., Koets A.P., Taverne M.A.M., and Verheijden J.H.M. 2007b. Postweaning growth check in pigs is markedly reduced by intermittent suckling and extended lactation. Journal of Animal Science 85: 258-266.

Black J.L., Mullan B.P., Lorschy M.L., and Giles L.R. 1993. Lactation in the sow during heat stress. Livestock Production Science 35: 153-170.

Bøe K. 1993. The Effect of Age at Weaning and Post-Weaning Environment on the Behaviour of Pigs. Acta Agriculturae Scandinavica, Section A - Animal Science 43: 173-180.

Bøe K. and Jensen P. 1995. Individual differences in suckling and solid food intake by piglets. Applied Animal Behaviour Science 42: 183-192.

Bohnenkamp A.L., Traulsen I., Meyer C., Müller K., and Krieter J. 2013. Group housing for lactating sows with electronically controlled crates: 1 . Reproductive traits, body condition, and feed intake. Journal of Animal Science 91: 3413-3419.

Bolhuis J., Oostindjer M., Van den Brand H., Gerrits W., and Kemp B. 2009. Voluntary feed intake in piglets: potential impact of early experience with flavours derived from the maternal diet. Voluntary feed intake in pigs. Wageningen, The Netherlands: Wageningen Pers: 37-61.

Bolhuis J.E., Oostindjer M., Hoeks C.W.F., de Haas E.N., Bartels A.C., Ooms M., and Kemp B. 2013. Working and reference memory of pigs (Sus scrofa domesticus) in a holeboard spatial discrimination task: the influence of environmental enrichment. Animal Cognition 16: 845-850.

Bolhuis J.E., Schouten W.G.P., Schrama J.W., and Wiegant V.M. 2005. Behavioural development of pigs with different coping characteristics in barren and 
substrate-enriched housing conditions. Applied Animal Behaviour Science 93: 213-228.

Bolhuis J.E., Schouten W.G.P., Schrama J.W., and Wiegant V.M. 2006. Effects of rearing and housing environment on behaviour and performance of pigs with different coping characteristics. Applied Animal Behaviour Science 101: 6885.

Broom D., Mendl M., and Zanella A. 1995. A comparison of the welfare of sows in different housing conditions. Animal science 61: 369-385.

Bruininx E., Binnendijk G., Van der Peet-Schwering C., Schrama J., Den Hartog L., Everts H., and Beynen A. 2002. Effect of creep feed consumption on individual feed intake characteristics and performance of group-housed weanling pigs. Journal of animal science 80: 1413-1418.

Burgwal-Konertz B. 1996. Das Saug- und Säugeverhalten bei der Gruppenhaltung abferkelnder und ferkelführender Sauen und ihren Würfen unter besonderer Berücksichtigung des Fremdsaugens. $\mathrm{PhD}$, Thesis $\mathrm{PhD}$, University of Hohenheim, Stuttgart, Germany.

Burri M., Wechsler B., Gygax L., and Weber R. 2009. Influence of straw length, sow behaviour and room temperature on the incidence of dangerous situations for piglets in a loose farrowing system. Applied Animal Behaviour Science 117: 181-189.

Chaloupková H., Illmann G., Bartoš L., and Špinka M. 2007. The effect of preweaning housing on the play and agonistic behaviour of domestic pigs. Applied Animal Behaviour Science 103: 25-34.

Chen C., Gilbert C.L., Yang G., Guo Y., Segonds-Pichon A., Ma J., Evans G., Brenig B., Sargent C., Affara N., and Huang L. 2008. Maternal infanticide in sows: Incidence and behavioural comparisons between savaging and non-savaging sows at parturition. Applied Animal Behaviour Science 109: 238-248.

Collins S.M., Surette M., and Bercik P. 2012. The interplay between the intestinal microbiota and the brain. Nature Reviews Microbiology 10: 735-742.

Cronin G.M., Barnett J.L., Hodge F.M., Smith J.A., and McCallum T.H. 1991. The welfare of pigs in two farrowing/lactation environments: cortisol responses of sows. Applied Animal Behaviour Science 32: 117-127. 
Cryan J.F. and O'Mahony S. 2011. The microbiome-gut-brain axis: from bowel to behavior. Neurogastroenterology \& Motility 23: 187-192.

D'Eath R.B. 2005. Socialising piglets before weaning improves social hierarchy formation when pigs are mixed post-weaning. Applied Animal Behaviour Science 93: 199-211.

D'Eath R., Arnott G., Turner S., Jensen T., Lahrmann H., Busch M., Niemi J.K., Lawrence A., and Sandøe P. 2014. Injurious tail biting in pigs: how can it be controlled in existing systems without tail docking? animal 8: 1479-1497.

Damm B.I., Forkman B., and Pedersen L.J. 2005. Lying down and rolling behaviour in sows in relation to piglet crushing. Applied Animal Behaviour Science 90: 3-20.

Danholt L., Moustsen V.A., Nielsen M.B.F., and Kristensen A.R. 2011. Rolling behaviour of sows in relation to piglet crushing on sloped versus level floor pens. Livestock Science 141: 59-68.

Dantzer R., Arnone M., and Mormede P. 1980. Effects of frustration on behaviour and plasma corticosteroid levels in pigs. Physiology and Behavior 24: 1-4.

Dardaillon M. 1986. Seasonal variations in habitat selection and spatial distribution of wild boar (Sus scrofa) in the Camargue, Southern France. Behavioural Processes 13: 251-268.

de Goede D., Gremmen B., Rodenburg T.B., Bolhuis J.E., Bijma P., Scholten M., and Kemp B. 2013. Reducing damaging behaviour in robust livestock farming. NJAS - Wageningen Journal of Life Sciences 66: 49-53.

de Jong I.C., Prelle I.T., van de Burgwal J.A., Lambooij E., Korte S.M., Blokhuis H.J., and Koolhaas J.M. 2000. Effects of environmental enrichment on behavioral responses to novelty, learning, and memory, and the circadian rhythm in cortisol in growing pigs. Physiology \& behavior 68: 571-578.

De Jonge F.H., Bokkers E.A.M., Schouten W.G.P., and Helmond F.A. 1996. Rearing piglets in a poor environment: Developmental aspects of social stress in pigs. Physiology and Behavior 60: 389-396.

Dexter N. 1999. The influence of pasture distribution, temperature and sex on homerange size of feral pigs in a semi-arid environment. Wildlife Research 26: 755762. 
Dickerson J. and Dobbing J. 1967. Prenatal and postnatal growth and development of the central nervous system of the pig. Proceedings of the Royal Society of London B: Biological Sciences 166: 384-395.

Dybkjær L. 1992. The identification of behavioural indicators of 'stress' in early weaned piglets. Applied Animal Behaviour Science 35: 135-147.

Edwards S., Brett M., Guy J., and Baxter E. 2011. Practical evaluation of an indoor free farrowing system: the PigSAFE pen. In: Proceedings of the 62nd Annual Meeting of the European Federation of Animal Science, 29 August-2 September, Stavanger, Norway. p 17.

Edwards S.A. 2002. Perinatal mortality in the pig: environmental or physiological solutions? Livestock Production Science 78: 3-12.

Figueroa J., Solà-Oriol D., Manteca X., and Pérez J.F. 2013. Social learning of feeding behaviour in pigs: Effects of neophobia and familiarity with the demonstrator conspecific. Applied Animal Behaviour Science 148: 120-127.

Fraser D. 1980. A review of the behavioural mechanism of milk ejection of the domestic pig. Applied Animal Ethology 6: 247-255.

Gerritsen R., Soede N.M., Langendijk P., Hazeleger W., and Kemp B. 2008. The intermittent suckling regimen in pigs: Consequences for reproductive performance of sows. Reproduction in Domestic Animals 43: 29-35.

Gonyou H.W., Beltranena E., Whittington D.L., and Patience J.F. 1998. The behaviour of pigs weaned at 12 and 21 days of age from weaning to market. Canadian Journal of Animal Science 78: 517-523.

Grimberg-Henrici C.G.E., Büttner K., Meyer C., and Krieter J. 2016a. Does housing influence maternal behaviour in sows? Applied Animal Behaviour Science 180: $26-34$

Grimberg-Henrici C.G.E., Vermaak P., Elizabeth Bolhuis J., Nordquist R.E., and van der Staay F.J. 2016b. Effects of environmental enrichment on cognitive performance of pigs in a spatial holeboard discrimination task. Animal Cognition 19: 271-283.

Gu Z., Xin H., Wang C., Shi Z., Liu Z., Yang F., Lin B., Wang C., and Li B. 2010. Effects of neoprene mat on diarrhea, mortality and foreleg abrasion of preweaning piglets. Preventive Veterinary Medicine 95: 16-22. 
Gundlach H. 1968. Brutfürsorge, Brutpflege, Verhaltensontogenese und Tagesperiodik beim Europäischen Wildschwein (Sus scrofa L.)1. Zeitschrift für Tierpsychologie 25: 955-995.

Gustafsson M., Jensen P., De Jonge F.H., Illmann G., and Spinka M. 1999. Maternal behaviour of domestic sows and crosses between domestic sows and wild boar. Applied Animal Behaviour Science 65: 29-42.

Hales J., Moustsen V.A., Nielsen M.B.F., and Hansen C.F. 2015. Temporary confinement of loose-housed hyperprolific sows reduces piglet mortality1. Journal of Animal Science 93: 4079-4088.

Harley S., Boyle L., O'Connell N., More S., Teixeira D., and Hanlon A. 2014. Docking the value of pigmeat? Prevalence and financial implications of welfare lesions in Irish slaughter pigs. Anim Welfare 23: 275-285.

Harris M.J., Li Y.Z., and Gonyou H.W. 2003. Savaging behaviour in gilts and sows. Canadian Journal of Animal Science 83: 819-821.

Heijtz R.D., Wang S., Anuar F., Qian Y., Björkholm B., Samuelsson A., Hibberd M.L., Forssberg H., and Pettersson S. 2011. Normal gut microbiota modulates brain development and behavior. Proceedings of the National Academy of Sciences 108: 3047-3052.

Held S., Mendl M., Devereux C., and Byrne R.W. 2000. Social tactics of pigs in a competitive foraging task: The 'informed forager' paradigm. Animal Behaviour 59: 569-576.

Held S.D.E. and Špinka M. 2011. Animal play and animal welfare. Animal Behaviour 81: 891-899.

Hessel E.F., Reiners K., and Van Den Weghe H.F.A. 2006. Socializing piglets before weaning: Effects on behavior of lactating sows, pre- and postweaning behavior, and performance of piglets. Journal of Animal Science 84: 28472855.

Hillmann E., Von Hollen F., Bünger B., Todt D., and Schrader L. 2003. Farrowing conditions affect the reactions of piglets towards novel environment and social confrontation at weaning. Applied Animal Behaviour Science 81: 99-109. 
Hoy S., Lutter C., Wähner M., and Puppe B. 1994. The effect of birth weight on the early postnatal vitality of piglets. DTW. Deutsche tierarztliche Wochenschrift 101: 393-396.

Hultén F., Dalin A.M., Lundeheim N., and Einarsson S. 1995a. Ovulation frequency among sows group-housed during late lactation. Animal Reproduction Science 39: 223-233.

Hultén F., Lundeheim N., Dalin A.M., and Einarsson S. 1995b. A field study on group housing of lactating sows with special reference to sow health at weaning. Acta Veterinaria Scandinavica 36: 201-212.

Hultén F., Wallenbeck A., and Rydhmer L. 2006. Ovarian activity and oestrous signs among group-housed, lactating sows: influence of behaviour, environment and production. Reprod Domest Anim 41.

Illmann G., Pokorná Z., and Špinka M. 2005. Nursing synchronization and milk ejection failure as maternal strategies to reduce allosuckling in pair-housed sows (Sus scrofa domestica). Ethology 111: 652-668.

Illmann G., Schrader L., Špinka M., and Šustr P. 2002. Acoustical mother-offspring recognition in pigs (sus scrofa domestica). Behaviour 139: 487-505.

Jarvis S., D'Eath R.B., Robson S.K., and Lawrence A.B. 2006. The effect of confinement during lactation on the hypothalamic-pituitary-adrenal axis and behaviour of primiparous sows. Physiology \& Behavior 87: 345-352.

Jensen P. 1986. Observations on the maternal behaviour of free-ranging domestic pigs. Applied Animal Behaviour Science 16: 131-142.

Jensen P. 1988. Maternal behaviour and mother-young interactions during lactation in free-ranging domestic pigs. Applied Animal Behaviour Science 20: 297-308.

Jensen P. 1995. The weaning process of free-ranging domestic pigs: within- and between-litter variations. Ethology 100: 14-25.

Jensen P., Florén K., and Hobroh B. 1987. Peri-parturient changes in behaviour in free-ranging domestic pigs. Applied Animal Behaviour Science 17: 69-76.

Jensen P. and Recén B. 1989. When to wean - Observations from free-ranging domestic pigs. Applied Animal Behaviour Science 23: 49-60.

Jensen P. and Redbo I. 1987. Behaviour during nest leaving in free-ranging domestic pigs. Applied Animal Behaviour Science 18: 355-362. 
Jensen P. and Stangel G. 1992. Behaviour of piglets during weaning in a seminatural enclosure. Applied Animal Behaviour Science 33: 227-238.

Jensen P., Stangel G., and Algers B. 1991. Nursing and suckling behaviour of seminaturally kept pigs during the first 10 days postpartum. Applied Animal Behaviour Science 31: 195-209.

Jensen P., Vestergaard K., and Algers B. 1993. Nestbuilding in free-ranging domestic sows. Applied Animal Behaviour Science 38: 245-255.

Kanaan V.T., Lay Jr D.C., Richert B.T., and Pajor E.A. 2012. Increasing the Frequency of Co-Mingling Piglets During the Lactation Period Alters the Development of Social Behavior Before and After Weaning. Journal of Applied Animal Welfare Science 15: 163-180.

Kemp B., Soede N.M., and Langendijk P. 2005. Effects of boar contact and housing conditions on estrus expression in sows. Theriogenology 63: 643-656.

Kilbride A.L., Mendl M., Statham P., Held S., Harris M., Cooper S., and Green L.E. 2012. A cohort study of preweaning piglet mortality and farrowing accommodation on 112 commercial pig farms in England. Preventive veterinary medicine 104: 281-291.

King M., Kelly D., Morel P., and Pluske J. 2003. Aspects of intestinal immunity in the pig around weaning. In: Pluske J., Le Dividich J., and Verstegen M. (eds.) Weaning the Pig: Concepts and Consequences. p 219. Wageningen Academic Publishers, The Netherlands.

Kirkden R., Broom D., and Andersen I. 2013. Invited review: piglet mortality: management solutions. Journal of animal science 91: 3361-3389.

Kongsted A.G. and Hermansen J.E. 2009. Induction of lactational estrus in organic piglet production. Theriogenology 72: 1188-1194.

Kuller W.I., Soede N.M., Bolhuis J.E., van Beers-Schreurs H.M.G., Kemp B., Verheijden J.H.M., and Taverne M.A.M. 2010. Intermittent suckling affects feeder visiting behaviour in litters with low feed intake. Livestock Science 127: 137-143.

Kuller W.I., Soede N.M., Van Beers-Schreurs H.M.G., Langendijk P., Taverne M.A.M., Kemp B., and Verheijden J.H.M. 2007. Effects of intermittent 
suckling and creep feed intake on pig performance from birth to slaughter. Journal of Animal Science 85: 1295-1301.

Kuller W.I., Soede N.M., van Beers-Schreurs H.M.G., Langendijk P., Taverne M.A.M., Verheijden J.H.M., and Kemp B. 2004. Intermittent suckling: Effects on piglet and sow performance before and after weaning. Journal of Animal Science 82: 405-413.

Kurvers R.H.J.M., Prins H.H.T., Van Wieren S.E., Van Oers K., Nolet B.A., and Ydenberg R.C. 2010a. The effect of personality on social foraging: Shy barnacle geese scrounge more. Proceedings of the Royal Society B: Biological Sciences 277: 601-608.

Kurvers R.H.J.M., van Oers K., Nolet B.A., Jonker R.M., van Wieren S.E., Prins H.H.T., and Ydenberg R.C. 2010b. Personality predicts the use of social information. Ecology Letters 13: 829-837.

Kutzer T., Bünger B., Kjaer J.B., and Schrader L. 2009. Effects of early contact between non-littermate piglets and of the complexity of farrowing conditions on social behaviour and weight gain. Applied Animal Behaviour Science 121: 16-24.

Laurenssen B., Strous J., van Nieuwamerongen S., Bolhuis J., van der Peet-Schwering C., and Soede N. 2016. Lactational oestrus in group housed lactating sows. In: 16th International Conference on Production Diseases in Farm Animals, Wageningen, The Netherlands

Lawrence A.B., Petherick J.C., McLean K., Gilbert C.L., Chapman C., and Russell J.A. 1992. Naloxone prevents interruption of parturition and increases plasma oxytocin following environmental disturbance in parturient sows. Physiology \& Behavior 52: 917-923.

Li Y., Johnston L., and Hilbrands A. 2010. Pre-weaning mortality of piglets in a bedded group-farrowing system. JSHAP 18: 75-80.

Maletínská J. and Špinka M. 2001. Cross-suckling and nursing synchronisation in group housed lactating sows. Applied Animal Behaviour Science 75: 17-32.

Maletínská J., Špinka M., Víchová J., and Stěhulová I. 2002. Individual recognition of piglets by sows in the early post-partum period. Behaviour 139: 975-991. 
Marchant J., Broom D., and Corning S. 2001. The influence of sow behaviour on piglet mortality due to crushing in an open farrowing system. Animal science 72: 19-28.

Marchant J.N. and Broom D.M. 1996. Factors affecting posture-changing in loosehoused and confined gestating sows. Animal Science 63: 477-485.

Mason G. and Latham N. 2004. Can't stop, won't stop: is stereotypy a reliable animal welfare indicator? Animal Welfare 13: S57-S70.

McGlone J.J. and Curtis S.E. 1985. Behavior and performance of weanling pigs in pens equipped with hide areas. Journal of Animal Science 60: 20-24.

Meynhardt H. 1980. Schwarzwild-Report: Vier Jahre unter Wildschweinen. Verlag J. Neumann-Neudamm, Berlin, Germany.

Miller H. and Slade R. 2003. Digestive physiology of the weaned pig. In: Pluske J., Le Dividich J., and Verstegen M. (eds.) Weaning the pig: concepts and consequences. Wageningen Academic Publishers, The Netherlands.

Moeser A.J., Vander Klok C., Ryan K.A., Wooten J.G., Little D., Cook V.L., and Blikslager A.T. 2007. Stress signaling pathways activated by weaning mediate intestinal dysfunction in the pig. American Journal of PhysiologyGastrointestinal and Liver Physiology 292: G173-G181.

Morgan C.A., Lawrence A.B., Chirnside J., and Deans L.A. 2001. Can information about solid food be transmitted from one piglet to another? Animal Science 73: 471-478.

Mota D., Alonso-Spilsbury M., Mayagoitia L., Trujillo M.E., Valencia J., and Ramírez-Necoechea R. 2002. Lactational estrus induction in the Mexican hairless sow. Animal Reproduction Science 72: 115-124.

Munsterhjelm C., Peltoniemi O.A.T., Heinonen M., Hälli O., Karhapää M., and Valros A. 2009. Experience of moderate bedding affects behaviour of growing pigs. Applied Animal Behaviour Science 118: 42-53.

Nannoni E., Valsami T., Sardi L., and Martelli G. 2014. Tail docking in pigs: a review on its short-and long-term consequences and effectiveness in preventing tail biting. Italian Journal of Animal Science 13: 3095.

Newberry R.C. and Wood-Gush D.G.M. 1985. The Suckling Behaviour of Domestic Pigs in a Semi-Natural Environment. Behaviour 95: 11-25. 
Olsen A.N.W., Dybkjær L., and Vestergaard K.S. 1998. Cross-suckling and associated behaviour in piglets and sows. Applied Animal Behaviour Science 61: 13-24.

Oostindjer M., Bolhuis J.E., Mendl M., Held S., van den Brand H., and Kemp B. 2011. Learning how to eat like a pig: Effectiveness of mechanisms for vertical social learning in piglets. Animal Behaviour 82: 503-511.

Oostindjer M., Kemp B., van den Brand H., and Bolhuis J.E. 2014. Facilitating 'learning from mom how to eat like a pig'to improve welfare of piglets around weaning. Applied Animal Behaviour Science 160: 19-30.

Oostindjer M., Muñoz J.M., Van den Brand H., Kemp B., and Bolhuis J.E. 2010. Maternal presence and environmental enrichment affect food neophobia of piglets. Biology letters: rsbl20100430.

Pajor E.A., Fraser D., and Kramer D.L. 1991. Consumption of solid food by suckling pigs: individual variation and relation to weight gain. Applied Animal Behaviour Science 32: 139-155.

Petersen V. 1994. The development of feeding and investigatory behaviour in freeranging domestic pigs during their first 18 weeks of life. Applied Animal Behaviour Science 42: 87-98.

Petersen V., Recén B., and Vestergaard K. 1990. Behaviour of sows and piglets during farrowing under free-range conditions. Applied Animal Behaviour Science 26: 169-179.

Pitts A.D., Weary D.M., Fraser D., Pajor E.A., and Kramer D.L. 2002. Alternative housing for sows and litters Part 5. Individual differences in the maternal behaviour of sows. Applied Animal Behaviour Science 76: 291-306.

Plomp M., Van de Pas L., and Verdoes N. 2016. Pigs on toilet (Innovatietraject ontwikkeling toiletstal 2011-2015; Eindconclusie - Inzichten voor de praktijk). http://edepot.wur.nl/359152.

Podgórski T., Lusseau D., Scandura M., Sönnichsen L., and Jędrzejewska B. 2014. Long-lasting, kin-directed female interactions in a spatially structured wild boar social network. PloS one 9: e99875.

Rantzer D., Svendsen J., and Weström B. 1995. Weaning of pigs raised in sowcontrolled and in conventional housing systems, 2: Behaviour studies and cortisol levels. Swedish Journal of Agricultural Research. 
Russo L., Massei G., and Genov P. 1997. Daily home range and activity of wild boar in a Mediterranean area free from hunting. Ethology Ecology \& Evolution 9: 287-294.

Saunders G. and McLeod S. 1999. Predicting home range size from the body mass or population densities of feral pigs, Sus scrofa (Artiodactyla: Suidae). Australian Journal of Ecology 24: 538-543.

Schrøder-Petersen D.L. and Simonsen H.B. 2001. Tail Biting in Pigs. The Veterinary Journal 162: 196-210.

Singer F.J., Otto D.K., Tipton A.R., and Hable C.P. 1981. Home ranges, movements, and habitat use of European wild boar in Tennessee. The Journal of Wildlife Management: 343-353.

Soede N. and Kemp B. 1996. Expression of oestrus and timing of ovulation in pigs. Journal of reproduction and fertility. Supplement 52: 91-103.

Soede N.M., Laurenssen B., Abrahamse-Berkeveld M., Gerritsen R., Dirx-Kuijken N., Langendijk P., and Kemp B. 2012. Timing of lactational oestrus in intermittent suckling regimes: Consequences for sow fertility. Animal Reproduction Science 130: 74-81.

Spinka M., Newberry R.C., and Bekoff M. 2001. Mammalian play: training for the unexpected. Quarterly Review of Biology: 141-168.

Stangel G. and Jensen P. 1991. Behaviour of semi-naturally kept sows and piglets (except suckling) during 10 days postpartum. Applied Animal Behaviour Science 31: 211-227.

Stolba A. and Wood-Gush D. 1989. The behaviour of pigs in a semi-natural environment. Animal production 48: 419-425.

Studnitz M., Jensen M.B., and Pedersen L.J. 2007. Why do pigs root and in what will they root?: A review on the exploratory behaviour of pigs in relation to environmental enrichment. Applied Animal Behaviour Science 107: 183-197.

Telkänranta H., Swan K., Hirvonen H., and Valros A. 2014. Chewable materials before weaning reduce tail biting in growing pigs. Applied Animal Behaviour Science 157: 14-22. 
Thomsson O., Bergqvist A.-S., Sjunnesson Y., Eliasson-Selling L., Lundeheim N., and Magnusson U. 2015. Aggression and cortisol levels in three different group housing routines for lactating sows. Acta Veterinaria Scandinavica 57: 9.

Thomsson O., Sjunnesson Y., Magnusson U., Eliasson-Selling L., Wallenbeck A., and Bergqvist A.-S. 2016. Consequences for piglet performance of group housing lactating sows at one, two, or three weeks post-farrowing. PloS one 11: e0156581.

Tuchscherer M., Puppe B., Tuchscherer A., and Tiemann U. 2000. Early identification of neonates at risk: Traits of newborn piglets with respect to survival. Theriogenology 54: 371-388.

Turpin D.L., Langendijk P., Chen T.-Y., and Pluske J.R. 2016. Intermittent suckling in combination with an older weaning age improves growth, feed intake and aspects of gastrointestinal tract carbohydrate absorption in pigs after weaning. Animals 6: 66.

Ursinus W.W., Van Reenen C.G., Kemp B., and Bolhuis J.E. 2014. Tail biting behaviour and tail damage in pigs and the relationship with general behaviour: Predicting the inevitable? Applied Animal Behaviour Science 156: 22-36.

Van den Brand H., Wamsteeker D., Oostindjer M., Kemp B., Bolhuis J.E., Van Enckevort L.C.M., and Van der Poel A.F.B. 2014. Effects of pellet diameter during and after lactation on feed intake of piglets pre- and postweaning. Journal of Animal Science 92: 4145-4153.

Van der Peet-Schwering C., Van Nieuwamerongen S., Bolhuis J., Troquet L., Hoofs A., and Soede N. 2015. Groepskraamsysteem: analyse van de ontwikkeling van zeugen, biggen en vleesvarkens.

van der Staay F.J., Gieling E.T., Pinzón N.E., Nordquist R.E., and Ohl F. 2012. The appetitively motivated " cognitive" holeboard: A family of complex spatial discrimination tasks for assessing learning and memory. Neuroscience and Biobehavioral Reviews 36: 379-403.

van Dixhoorn I.D., Reimert I., Middelkoop J., Bolhuis J.E., Wisselink H.J., Koerkamp P.W.G., Kemp B., and Stockhofe-Zurwieden N. 2016. Enriched Housing Reduces Disease Susceptibility to Co-Infection with Porcine Reproductive and Respiratory Virus (PRRSV) and Actinobacillus 
pleuropneumoniae (A. pleuropneumoniae) in Young Pigs. PloS one 11: e0161832.

van Wettere W.H.E.J., Kaisler-Smith C.R., Terry R., Weaver A.C., Herde P.J., Kennaway D.J., Hughes P.E., and Kind K.L. 2013. Boar contact is an effective stimulant of ovulation during early lactation. Livestock Science 155: 454-458.

Vasdal G., Glærum M., Melišová M., Bøe K.E., Broom D.M., and Andersen I.L. 2010. Increasing the piglets' use of the creep area-A battle against biology? Applied Animal Behaviour Science 125: 96-102.

Vente-Spreeuwenberg M. and Beynen A. 2003. Diet-mediated modulation of small intestinal integrity in weaned piglets. In: Pluske J., Le Dividich J., and Verstegen M. (eds.) Weaning the pig: concepts and consequences. p 145-198.

Weary D.M., Jasper J., and Hötzel M.J. 2008. Understanding weaning distress. Applied Animal Behaviour Science 110: 24-41.

Weary D.M., Pajor E.A., Bonenfant M., Fraser D., and Kramer D.L. 2002. Alternative housing for sows and litters Part 4. Effects of sow-controlled housing combined with a communal piglet area on pre- and post-weaning behaviour and performance. Applied Animal Behaviour Science 76: 279-290.

Weary D.M., Phillips P.A., Pajor E.A., Fraser D., and Thompson B.K. 1998. Crushing of piglets by sows: effects of litter features, pen features and sow behaviour. Applied Animal Behaviour Science 61: 103-111.

Weber R., Keil N., Fehr M., and Horat R. 2007. Piglet mortality on farms using farrowing systems with or without crates. Animal Welfare 16: 277-279.

Weber R., Keil N.M., Fehr M., and Horat R. 2009. Factors affecting piglet mortality in loose farrowing systems on commercial farms. Livestock Science 124: 216222.

Wechsler B. 1996. Rearing pigs in species-specific family groups. Animal Welfare 5: 25-35.

Wechsler B. and Hegglin D. 1997. Individual differences in the behaviour of sows at the nest-site and the crushing of piglets. Applied Animal Behaviour Science 51: 39-49. 
Wechsler B. and Weber R. 2007. Loose farrowing systems: challenges and solutions. Animal Welfare 16: 295-307.

Weng R.C., Edwards S.A., and English P.R. 1998. Behaviour, social interactions and lesion scores of group-housed sows in relation to floor space allowance. Applied Animal Behaviour Science 59: 307-316.

Westin R., Hultgren J., and Algers B. 2015. Strategic use of straw increases nest building in loose housed farrowing sows. Applied Animal Behaviour Science 166: 63-70.

Widowski T.M., Torrey S., Bench C.J., and Gonyou H.W. 2008. Development of ingestive behaviour and the relationship to belly nosing in early-weaned piglets. Applied Animal Behaviour Science 110: 109-127.

Wijtten P.J., van der Meulen J., and Verstegen M.W. 2011. Intestinal barrier function and absorption in pigs after weaning: a review. British journal of nutrition 105: 967-981.

Wischner D., Kemper N., and Krieter J. 2009. Nest-building behaviour in sows and consequences for pig husbandry. Livestock Science 124: 1-8.

Yajun G., Baoming L., Mingli L., Baozhong L., and Zhaobing G. 2011. Impacts of room temperature on sow behaviour and creep box usage for pre-weaning piglet. Transactions of the Chinese Society of Agricultural Engineering 2011.

Yun J., Swan K.-M., Farmer C., Oliviero C., Peltoniemi O., and Valros A. 2014. Prepartum nest-building has an impact on postpartum nursing performance and maternal behaviour in early lactating sows. Applied Animal Behaviour Science 160: 31-37.

Yun J., Swan K.-M., Oliviero C., Peltoniemi O., and Valros A. 2015. Effects of prepartum housing environment on abnormal behaviour, the farrowing process, and interactions with circulating oxytocin in sows. Applied Animal Behaviour Science 162: 20-25.

Yun J. and Valros A. 2015. Benefits of prepartum nest-building behaviour on parturition and lactation in sows-a review. Asian-Australasian journal of animal sciences 28: 1519 . 
Zoric M., Nilsson E., Lundeheim N., and Wallgren P. 2009. Incidence of lameness and abrasions in piglets in identical farrowing pens with four different types of floor. Acta Veterinaria Scandinavica 51: 23.

Zoric M., Nilsson E., Mattsson S., Lundeheim N., and Wallgren P. 2008. Abrasions and lameness in piglets born in different farrowing systems with different types of floor. Acta Veterinaria Scandinavica 50: 37.

Zoric M., Sjölund M., Persson M., Nilsson E., Lundeheim N., and Wallgren P. 2004. Lameness in piglets. Abrasions in nursing piglets and transfer of protection towards infections with Streptococci from sow to offspring. J Vet Med B 51. 



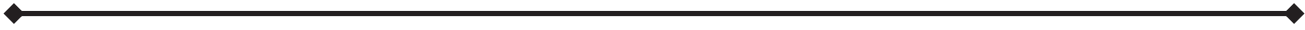

Summary

$\longleftrightarrow$ 
Animal welfare concerns have led to a ban of individual confinement of sows during the major part of gestation in the EU from 2013 onwards (Council Directive 2001/88/EC). Also outside the EU, for instance in the USA, there is a move toward group housing of gestating sows. During farrowing and lactation, however, most sows (around 95\% of sows in Europe) are still housed individually in a farrowing crate, in which they cannot turn around. This conventional farrowing system provides a large contrast with the, in the EU mandatory, group housing during gestation, with abrupt transitions in the degree of freedom of movement and social contact. Conventional housing is being criticised not only because it impairs sow welfare, but also because it provides a suboptimal environment for piglet development (e.g. a relatively small and barren pen with limited contact with non-littermates). This environment is in large contrast with natural conditions, in which pigs live in groups of several females and their offspring in a diverse habitat. In a conventional system, problems with piglet performance mainly occur after weaning, when the piglets are separated from the sow and have to cope with a dietary change -from a mainly milk-based diet to (solid) piglet feed-, relocation to a nursery and, often, mixing with unfamiliar piglets. The piglets are generally not well prepared for these simultaneous abrupt changes that occur at a relatively young age (mostly 3 to 4 weeks), which is reflected in poor postweaning performance. This poor performance is characterised by a low feed intake, impaired intestinal function, a growth check, diarrhoea, and behavioural indicators of distress.

We have developed an alternative farrowing system that more closely resembles the natural situation, compared with a conventional farrowing system. This alternative multi-suckling (MS) system houses a group of five sows and their piglets together in a more spacious and enriched environment. The behaviour of domestic pigs kept under (semi-)natural conditions still closely resembles that of their wild ancestors and piglet development may benefit from stimulating the expression of natural behaviour. Therefore, the main aim of this thesis was to investigate the development of pigs that were raised in the MS system, with a focus on the transition around weaning and performance later in life. This thesis is part of a larger project with an overall goal to develop a commercially feasible group housing system for sows and their piglets, with the natural behaviour of pigs as the starting point. 
As a first step, a literature review on group housing systems for lactating sows was conducted, to gain more insight into the success and risk factors that may be encountered in the development of such systems (Chapter 2). A main finding was that gradual transitions in social and physical environment around gestation, farrowing, grouping of sows and litters, and weaning seemed to be key success factors for animal performance in group housing systems during lactation. We based the design and management of our MS system on the insights from Chapter 2. Our MS system consisted of five pens with nesting material -in which sows could farrow individually without being crated-, connected with a communal area. The latter was divided in an area for general activity and resting, a communal feeding area, and a dunging area. At approximately one week of age, piglets were allowed to access the communal area and were thus able to mingle with the other litters and sows. In the communal feeding area, piglets could eat together with the sows and they could also eat piglet feed in an area accessible to the piglets only. Enrichment in the form of straw, hessian sacks, and ropes was provided throughout the system. In a second version of the system, an area was included which was only accessible to the sows, to enable investigation of a more gradual weaning procedure.

As our MS system has features that can ease the transition to the post-weaning phase (e.g. stimulation of social development and experience with solid feed), we compared the pre- and post-weaning development of piglets raised in the MS system with that of conventionally raised piglets in Chapter 3. This study encompassed a system comparison from birth until 9 weeks of age. Piglets were weaned at 4 weeks of age, and post-weaning housing consisted of an enriched pen with 40 piglets for the piglets raised in the MS system, and of a standard nursery pen with 10 littermates for the conventionally raised piglets. Piglets in the MS system showed a higher frequency of feed-directed behaviour at 2 weeks of age than the conventionally housed piglets ( $6.80 \pm 0.96$ vs. $2.35 \pm$ 0.59 occurrences per hour, $P<0.01$ ), suggesting an earlier start of feed exploration. This was possibly due to social learning of eating behaviour from the sows and other piglets in the communal feeding area. Moreover, MS piglets showed less damaging oral manipulation (e.g. tail biting) before weaning than conventionally housed piglets $(1.4 \pm 0.2$ vs. $3.6 \pm 0.3$ occurrences per hour, $P<$ 0.001 ), which was likely related with the more enriched environment in the MS system. In the first 2 weeks after weaning, piglets that were raised in the MS 
system showed a better performance than the piglets raised in the conventional system. Piglets raised in the MS system had fewer aggression-related skin lesions, showed a lower peak in belly-nosing behaviour, had more solid faecal consistency scores -indicating a lower occurrence of diarrhoea-, ate $81 \%$ more feed between days 1 and 2 post-weaning, and had an $82 \%$ higher weight gain until day 5 , despite a similar weaning weight ( $8.4 \pm 0.2$ vs. $8.3 \pm 0.2 \mathrm{~kg})$. In addition, over the entire nursery phase between 4 and 9 weeks of age, MSreared piglets showed a higher frequency of play behaviour ( $4.0 \pm 0.3$ vs. $2.8 \pm$ 0.3 occurrences per hour, $P<0.05)$ and a lower frequency of damaging oral manipulation $(1.8 \pm 0.3$ vs. $3.5 \pm 0.4$ occurrences per hour, $P<0.01)$ than the conventionally reared piglets. Altogether, this suggested that the combination of the pre-weaning and post-weaning socially and environmentally enriched environment benefitted piglet development until 9 weeks of age, compared with conventional housing.

Pre-weaning mortality was, however, higher in the MS system than in the conventional system $(3.22 \pm 0.42$ vs. $1.52 \pm 0.25$ piglets died per litter, $P<$ 0.01 ), mainly due to crushing before grouping of litters. In an attempt to limit mortality, changes to the design of the farrowing pens were made in the second version of the MS system, which was used for all follow-up studies. In Chapters 4 and 5, studies were described with a similar weaning age and contrast in pre-weaning housing conditions as in Chapter 3. However, unlike in Chapter 3, post-weaning housing was equal for both treatment groups and consisted of relatively large pens bedded with peat, sawdust and straw, which housed 4 litter-mates each. The study in Chapter $\mathbf{4}$ gave some indication that the differences in post-weaning performance observed in Chapter 3 may partly be explained by a difference in aspects of intestinal function. In Chapter 4 , we found that pre-weaning housing affected measures of post-weaning carbohydrate absorption. Piglets reared in the MS system had lower plasma concentrations of mannitol ( $320 \pm 116$ vs. $592 \pm 120 \mathrm{nmol} / \mathrm{mL}, P=0.04)$ and galactose $(91 \pm 18$, vs. $157 \pm 19 \mathrm{nmol} / \mathrm{mL}, P=0.04)$ than conventionally reared piglets, 20 minutes after oral administration on day 5 post-weaning. In addition, piglets reared in the MS system seemed to have a better feed utilisation than conventionally reared piglets in the early post-weaning period, as piglets reared in the MS system gained more weight than conventionally reared piglets between days 2 to 5 post-weaning $(0.67 \pm 0.12 \mathrm{~kg}$ vs. $0.39 \pm 0.16$ $\mathrm{kg}, P=0.02)$, despite a similar feed intake. Overall, no differences were found 
between weaning and two weeks thereafter, in feed intake (day 0 to 13: $4.61 \pm$ 0.24 vs. $4.56 \pm 0.20 \mathrm{~kg}$ per piglet), weight gain (day -1 to $13: 3.27 \pm 0.16$ vs. $3.00 \pm 0.38 \mathrm{~kg}$ per piglet) and faecal consistency scores between piglets raised in the MS system or in the conventional system, respectively. This is in contrast with the findings in Chapter 3, and may indicate that the enriched post-weaning conditions in Chapter 4 reduced the contrast between piglets raised pre-weaning in either the MS system or the conventional system. The piglets used in Chapter 4 were studied further in Chapter 5, with a focus on their cognitive and social performance, assessed using behaviour tests in a competitive context. It was hypothesised that pigs reared in the MS system would be better prepared to deal with social and cognitive challenges later in life, as a result of their more complex social and physical rearing environment. In a feed competition test, piglets from both pre-weaning housing systems showed a similar frequency of aggressive behaviours. Unexpectedly, the conventionally reared piglets tended to establish more clear dominance relationships than the piglets reared in the MS system, as conventionally reared piglets e.g. showed more asymmetry within the tested pairs in the number of access bouts to the feed $(1.8 \pm 0.3$ vs. $0.8 \pm 0.2, P<0.05)$. This may indicate that the more dominant pig defended the feed better against the submissive pig in conventionally reared pairs than in MS-reared pairs. Possibly, these findings were related with a difference in the context of obtaining feed in both preweaning farrowing systems; in the MS system, piglets were stimulated to eat together in the communal feeding area, whereas in the conventional system, piglets had only one small piglet feeder for the whole litter and therefore had a more competitive environment to obtain solid feed. In the subsequent 'informed forager test', aspects of spatial learning and foraging strategies in a competitive context were measured. During individual training, submissive (informed) pigs learned to remember the location of a food reward in a testing arena with 8 buckets (the same bucket was baited in a search visit and a subsequent relocation visit), whereas dominant (non-informed) pigs always found the bait in a random bucket (search visits only). Pigs from both preweaning housing systems performed similarly in learning their task. After individual training, the informed pigs' individual search visit was followed by a pairwise relocation visit in which they were accompanied by the non-informed pig. During these paired visits, we found indications that pigs raised in the MS system made more use of social information from their partner than pigs raised 
in the conventional system. For instance, among MS-reared pairs, the percentage of testing trials in which the non-informed pig ate from the baited bucket was positively correlated with the number of times the non-informed pig displaced its informed partner from a bucket $(\mathrm{r}=0.84, P=0.01)$. On the other hand, among conventionally raised pairs, the percentage of trials in which a non-informed pig ate from the baited bucket was positively correlated with the mean number of buckets the non-informed pig visited itself per testing trial $(\mathrm{r}=0.77, P=0.03)$.

During the last experiment described in this thesis, we focussed on the weaning procedure within the MS system (Chapter 6), as problems associated with weaning are not only related with pre- and post-weaning housing conditions, but also with the timing and the abruptness of weaning itself. Development from birth until slaughter was studied in two groups that differed in treatment between 4 to 9 weeks of age. Between 0 to 4 weeks of age, both groups were housed in the MS system. Thereafter, pigs were either subjected to abrupt weaning at 4 weeks of age and subsequently housed in a nursery, or subjected to a more gradual weaning procedure during an extended lactation period of 9 weeks. The gradual weaning procedure included forced intermittent-suckling in the fifth week of lactation (during which sows were separated from the piglets for 10 hours daily), after which sows could voluntarily separate themselves from the piglets. The use of forced intermittent-suckling (in combination with exposure to a boar) also aimed to stimulate lactational oestrus and ovulation, so that sows could be inseminated during lactation. Thereby, the yearly reproductive output of the sows may be maintained, despite the extended lactation period. At 9 weeks of age, both groups were relocated to a finishing unit where they were housed under equal conditions. We found that abrupt weaning at 4 weeks of age seemed to have more impact than forced IS followed by voluntary separation by the sows. Within the first 2 weeks after starting the different weaning procedures, weaned piglets showed a reduction in average daily gain and a distinct peak in belly-nosing behaviour (with an 8fold higher frequency of belly nosing at 41 days of age), which were not shown by the piglets remaining in the MS system. This may reflect difficulties coping with the transition from a milk-based diet to a diet of only solid feed in the weaned piglets. In addition, the percentage of pigs scored as having pasty or watery faeces, was twice as high in the group of weaned pigs, indicating a numerically higher occurrence of diarrhoea. In contrast, for the piglets weaned 
at 9 weeks of age, weaning did not result in a growth check or any behavioural indicators of having difficulty coping with the post-weaning situation. In addition, feed intake after transition to the finishing unit was similar in both treatments, indicating that pigs weaned with the more gradual procedure had a more successful transition to a diet of only solid feed.

Interestingly, although differences in production performance disappeared in the finishing phase, we found several long-term beneficial effects of the gradual weaning treatment on behaviour. During the finishing phase, belly-nosing persisted at higher levels in pigs weaned at 4 weeks of age, whereas pigs weaned at 9 weeks of age performed almost no belly-nosing (ranging from a 4 to 16-fold higher occurrence over the 3 observation days during the finishing phase). Additionally, in the tenth week of the finishing phase, pigs subjected to the gradual weaning treatment had less damage on the skin related with aggression and oral manipulation. The latter was supported by lower levels of damaging manipulative behaviour from 4 weeks of age up until the finishing phase, compared with abrupt weaning at a younger age (1.2 \pm 0.2 vs. $1.8 \pm 0.2$ occurrences per hour, $P<0.05$ ). This may reflect the smoother transitions around week 4 and 9 for the pigs subjected to the more gradual weaning treatment.

To conclude, our results support the expectation that the MS system would promote piglet development, compared with conventional housing, and that a more gradual weaning procedure during an extended lactation in the MS system would further enhance piglet development, compared with abrupt weaning at 4 weeks of age. We found differences in performance before weaning, around weaning-related transition periods, and later in life, which were reflected in behaviour, physical lesions, production performance, and measures related with intestinal function. Pre-weaning piglet mortality was, however, an issue in the MS system, which was likely the result of a suboptimal farrowing environment in combination with the use of a type of sow that may perform well in a conventional system but may be less suitable for alternative farrowing systems such as the MS system.

In general, enabling gradual transitions throughout life, via housing and management, seem important for sow and piglet performance. Specifically in relation to alleviation of weaning-related issues encountered in conventional 
systems, the MS system can contribute by i) better pre-weaning preparation of the piglets for the moment of weaning by providing a more stimulating social and physical environment, ii) eliminating or reducing the number of concurrent stressors at weaning, and iii) enabling a more gradual weaning procedure during an extended lactation. 


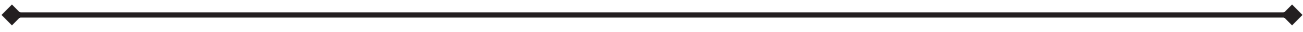

Samenvatting

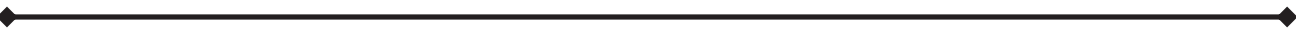


Maatschappelijke zorgen over dierenwelzijn hebben geleid tot een verbod op het individueel huisvesten van zeugen tijdens het grootste deel van de dracht in de EU vanaf 2013 (Richtlijn 2001/88/EG van de Raad van 23 oktober 2001). Ook buiten de EU, bijvoorbeeld in de VS, worden dragende zeugen steeds vaker in groepen gehuisvest. Tijdens het werpen en de daaropvolgende zoogperiode wordt het merendeel van de zeugen echter nog steeds individueel gehuisvest in een kraambox, waarin de zeugen zich niet kunnen omdraaien (circa 95\% van de zeugen in Europa). Deze manier van huisvesten vormt daarmee een groot contrast met de groepshuisvesting tijdens de drachtfase, met abrupte overgangen in de mate van bewegingsvrijheid en sociaal contact. Het gangbare kraamsysteem is niet alleen nadelig voor het welzijn van de zeugen, maar biedt ook een suboptimale omgeving voor de ontwikkeling van de biggen (bijvoorbeeld een relatief klein en kaal hok waarin biggen beperkt contact hebben met biggen uit andere tomen). Een dergelijke omgeving staat in groot contrast met de omstandigheden in de natuur, waarin varkens in een diverse habitat leven in groepen die bestaan uit een aantal zeugen en hun nakomelingen. In het gangbare systeem ondervinden biggen vaak problemen nadat ze zijn gespeend. Bij het spenen worden de biggen gescheiden van de zeug en moeten de biggen omschakelen van een dieet dat voornamelijk uit melk bestaat, naar biggenvoer. Daarnaast worden de biggen overgeplaatst naar een opfokstal en worden ze vaak gemengd met onbekende biggen. Biggen zijn over het algemeen slecht voorbereid op deze gelijktijdige en abrupte veranderingen die plaatsvinden op een relatief jonge leeftijd (rond 3 tot 4 weken). De problemen die na spenen ontstaan kenmerken zich door een lage voeropname, een verminderde functie van het maagdarmkanaal, een vermindering in groei, diarree, en stress-gerelateerd gedrag.

Wij hebben een alternatief kraamsysteem ontwikkeld dat, vergeleken met het gangbare systeem, meer lijkt op de natuurlijke situatie. In dit alternatieve groepskraamsysteem worden 5 zeugen en hun biggen samen gehuisvest in een grotere en meer verrijkte omgeving. Het gedrag van gedomesticeerde varkens dat onder (semi-)natuurlijke omstandigheden wordt gehouden lijkt nog steeds sterk op dat van hun wilde voorouders, en de ontwikkeling van biggen kan baat hebben bij het stimuleren van natuurlijk gedrag. Daarom is het hoofddoel van dit proefschrift om de ontwikkeling van varkens te onderzoeken die zijn opgegroeid in het groepskraamsysteem, met een focus op de overgangsfase rondom spenen en hun functioneren in het latere leven. Dit proefschrift is 
onderdeel van een groter project dat als overkoepelend doel heeft om een commercieel toepasbaar groepshuisvestingssysteem voor kraamzeugen en hun biggen te ontwikkelen, met het natuurlijke gedrag van varkens als uitgangspunt.

Om meer inzicht te krijgen in de succes- en risicofactoren die een rol zouden kunnen spelen in de ontwikkeling van een groepskraamsysteem, is een literatuuronderzoek gedaan naar dergelijke systemen (Hoofdstuk 2). Een belangrijke bevinding was dat geleidelijke overgangen in de sociale en fysieke omgeving rond dracht, werpen, groeperen van de zeugen en hun biggen, en spenen kritieke succesfactoren lijken voor een goede prestatie van de dieren in groepshuisvestingssystemen voor kraamzeugen. Het ontwerp en management van ons groepskraamsysteem is gebaseerd op de inzichten die zijn verkregen uit het literatuuroverzicht. Ons groepskraamsysteem bestond uit 5 kraamhokken met nestbouwmateriaal (waarin zeugen individueel konden werpen, zonder vast te staan in een kraambox), met aansluitend een gezamenlijke ruimte. De gezamenlijke ruimte was ingedeeld in gebieden voor rusten en algemene activiteit, een mee-eetruimte, en een mestruimte. De biggen kregen toegang tot de gezamenlijke ruimte en andere kraamhokken rond een leeftijd van 1 week. In de mee-eetruimte konden de biggen samen eten met de zeugen en hadden de biggen ook toegang tot biggenvoer in een ruimte die alleen voor de biggen bereikbaar was. In het hele systeem was verrijkingsmateriaal aanwezig ter stimulering van exploratie- en foerageergedrag, in de vorm van stro, jute zakken, en touwen. De tweede versie van het groepskraamsysteem bevatte daarnaast een ruimte die alleen toegankelijk was voor de zeugen, om de toepassing van een meer geleidelijk speenproces te onderzoeken.

Aangezien ons groepskraamsysteem elementen bevat die de overgang naar de periode na spenen kunnen vergemakkelijken (bijvoorbeeld stimulering van sociale ontwikkeling en het opdoen van ervaring met vast voer), hebben we in Hoofdstuk 3 zowel voor als na spenen de ontwikkeling van biggen die waren opgegroeid in het groepskraamsysteem vergeleken met die van biggen uit een gangbaar kraamsysteem. Deze studie bestond uit een systeemvergelijking van geboorte tot 9 weken leeftijd. Biggen werden op 4 weken leeftijd gespeend en huisvesting na spenen bestond uit een verrijkt hok met 40 biggen voor de dieren die waren opgegroeid in het groepskraamsysteem, en uit standaard 
huisvesting in een opfokhok met 10 toomgenoten voor de dieren die uit een gangbaar kraamhok kwamen. Vóór spenen lieten biggen in het groepskraamsysteem vaker voergericht gedrag zien (bijvoorbeeld het besnuffelen of eten van zeugen- of biggenvoer) dan de gangbaar gehuisveste biggen (6.80 \pm 0.96 vs. $2.35 \pm 0.59$ keer per uur op 2 weken leeftijd, $P<0.01$ ). Dit suggereert dat de groepskraambiggen eerder begonnen met het exploreren van vast voer. Mogelijk kwam dit door de mogelijkheid om te leren eten van de zeugen en andere biggen in de mee-eetruimte. Verder lieten de groepskraambiggen vóór spenen minder vaak beschadigend gedrag zien, zoals oor- en staartbijten, vergeleken met de gangbaar gehuisveste biggen $(1.4 \pm 0.2$ vs. $3.6 \pm 0.3$ keer per uur, $P<0.001)$. Dit was waarschijnlijk gerelateerd aan de meer verrijkte omgeving in het groepskraamsysteem. In de eerste 2 weken na spenen presteerden de groepskraambiggen beter dan de biggen die onder gangbare omstandigheden waren opgegroeid. De groepskraambiggen hadden minder huidbeschadigingen die aan agressie gerelateerd zijn, lieten een lagere piek in 'belly-nosing' gedrag zien (maladaptief gedrag waarbij biggen de buik van een hokgenoot masseren), hadden een vastere mestconsistentie (indicatief voor minder diarree), aten $81 \%$ meer voer tussen dag 1 en 2 na spenen, en hadden een $82 \%$ hogere gewichtstoename tot dag 5 na spenen ondanks een vergelijkbaar speengewicht $(8.4 \pm 0.2$ vs. $8.3 \pm 0.2 \mathrm{~kg})$. Verder lieten de groepskraambiggen vaker spelgedrag zien ( $4.0 \pm 0.3$ vs. $2.8 \pm 0.3$ keer per uur, $P<0.05)$, terwijl ze minder vaak beschadigend gedrag vertoonden dan de gangbaar gehuisveste biggen $(1.8 \pm 0.3$ vs. $3.5 \pm 0.4$ keer per uur, $P<0.01)$ gedurende de gehele opfokfase tussen 4 en 9 weken leeftijd. Gezamenlijk suggereren deze resultaten dat de ontwikkeling van de biggen tot een leeftijd van 9 weken werd gestimuleerd door de combinatie van de sociaal en fysiek meer verrijkte omgeving voor en na spenen, in vergelijking met de gangbare huisvestingscondities.

Vóór spenen was de sterfte van biggen echter hoger in het groepskraamsysteem dan in het gangbare kraamsysteem $(3.22 \pm 0.42$ vs. $1.52 \pm 0.25$ uitgevallen biggen per toom, $P<0.01$ ). Dit kwam voornamelijk door het doodliggen van biggen door de zeugen, voordat de tomen gegroepeerd werden (dus binnen 1 week na geboorte). In een poging om de uitval te verlagen, hebben we het ontwerp van de kraamhokken aangepast in de tweede versie van het systeem, dat gebruikt is voor alle vervolgstudies. In de studies die zijn beschreven in Hoofdstuk 4 en 5 was het contrast in huisvesting vóór spenen en de 
speenleeftijd vergelijkbaar met het contrast in Hoofdstuk 3 (groepskraamsysteem vs. gangbaar systeem tot 4 weken leeftijd). In tegenstelling tot Hoofdstuk 3 was de huisvesting na spenen hetzelfde voor beide behandelingsgroepen, en deze bestond uit relatief grote hokken met turf, zaagsel, en stro voor 4 toomgenoten per hok. Het experiment beschreven in Hoofdstuk 4 geeft een indicatie dat de verschillen in prestatie na spenen beschreven in Hoofdstuk 3 deels verklaard zouden kunnen worden door verschillen in darmfunctie. In Hoofdstuk 4 vonden we dat huisvesting vóór spenen invloed had op aspecten van koolhydraatabsorptie na spenen. Vergeleken met gangbaar opgegroeide dieren, hadden biggen die waren opgegroeid in het groepskraamsysteem op dag 5 na spenen lagere concentraties mannitol $(320 \pm 116$ vs. $592 \pm 120 \mathrm{nmol} / \mathrm{mL}, P=0.04)$ en galactose $(91 \pm 18$, vs. $157 \pm 19 \mathrm{nmol} / \mathrm{mL}, P=0.04)$ in hun bloed op 20 minuten na orale toediening. Daarnaast hadden de groepskraambiggen een hogere gewichtstoename tussen dag 2 en 5 na spenen $(0.67 \pm 0.12 \mathrm{~kg}$ vs. $0.39 \pm 0.16$ $\mathrm{kg}, \quad P=0.02)$, terwijl de voeropname vergelijkbaar was voor beide behandelingsgroepen. In de eerste 2 weken na spenen waren er over het geheel genomen geen verschillen in voeropname (dag 0 tot $13: 4.61 \pm 0.24$ vs. $4.56 \pm$ $0.20 \mathrm{~kg}$ per big), gewichtstoename (dag -1 tot $13: 3.27 \pm 0.16$ vs. $3.00 \pm 0.38 \mathrm{~kg}$ per big), en mestconsistentie tussen biggen uit respectievelijk het groepskraamsysteem en uit het gangbare kraamsysteem. Dit is tegenstrijdig met de bevindingen uit Hoofdstuk 3, wat mogelijk aangeeft dat de verrijkte huisvesting na spenen in Hoofdstuk 4 het contrast tussen behandelingsgroepen heeft verminderd.

De biggen die in Hoofdstuk 4 zijn onderzocht, zijn verder bestudeerd in Hoofdstuk 5, waarbij de focus lag op de sociale en cognitieve ontwikkeling van de dieren. Dit werd onderzocht met behulp van gedragstesten waarin biggen met elkaar moesten concurreren voor toegang tot een voerbeloning. We verwachtten dat de groepskraambiggen beter voorbereid zouden zijn op sociale en cognitieve uitdagingen op latere leeftijd, als gevolg van hun verrijkte opgroeicondities met een complexere fysieke en sociale omgeving. Tijdens een voercompetitietest lieten de biggen uit beide kraamsystemen een vergelijkbare frequentie van agressief gedrag zien. Tegen de verwachting in leken de biggen die waren opgegroeid in het gangbare kraamsysteem echter een duidelijkere dominantiehiërarchie te hebben dan de groepskraambiggen. Binnen de geteste paren lieten de dieren uit het gangbare systeem bijvoorbeeld meer asymmetrie 
zien in het aantal keer dat een big toegang had tot het voer $(1.8 \pm 0.3$ vs. $0.8 \pm$ $0.2, P<0.05)$. Dit zou erop kunnen wijzen dat de dominantere big het voer beter verdedigde tegen de onderdanige big binnen gangbaar opgegroeide paren dan binnen paren uit het groepskraamsysteem. Deze bevindingen zijn mogelijk gerelateerd aan verschillen in de context van voeropname in beide kraamsystemen. In het groepskraamsysteem werden de biggen gestimuleerd om samen te eten in de mee-eetruimte, terwijl de biggen in het gangbare kraamsysteem mogelijk meer concurrentie ondervonden aangezien ze slechts één relatief kleine voerbak beschikbaar hadden voor de hele toom.

In de opvolgende gedragstest (de 'informed forager test') werden aspecten van ruimtelijk leren en foerageerstrategieën gemeten. Tijdens de individuele trainingsfase leerden onderdanige (geïnformeerde) biggen de locatie van een voerbeloning te onthouden in een testruimte met 8 emmers (de beloning werd in dezelfde emmer geplaatst tijdens 2 opeenvolgende bezoeken aan de testruimte). Dominante (niet- geïnformeerde) biggen vonden hun voerbeloning altijd in een willekeurige emmer tijdens elk opeenvolgend bezoek. De biggen uit beide kraamsystemen presteerden vergelijkbaar in het leren van hun taak. $\mathrm{Na}$ de individuele training werd het eerste individuele bezoek van de geïnformeerde big gevolgd door een paarsgewijs bezoek waarin de big werd vergezeld door zijn niet-geïnformeerde partner. Tijdens deze paarsgewijze bezoeken vonden we aanwijzingen dat de groepskraambiggen meer gebruik maakten van sociale informatie van hun partner dan biggen die waren opgegroeid in het gangbare kraamsysteem. Bijvoorbeeld, onder groepskraambiggen was het percentage tests waarin de niet-geïnformeerde big van de beloning at positief gecorreleerd met het aantal keer dat de nietgeïnformeerde big zijn geïnformeerde partner verdrong van een emmer $(\mathrm{r}=$ 0.84, $P=0.01)$. Onder gangbaar opgegroeide dieren was het percentage tests waarin de niet-geïnformeerde big van de beloning at echter positief gecorreleerd met het gemiddeld aantal emmers dat de big zelf bezocht $(\mathrm{r}=$ $0.77, P=0.03)$.

Tijdens het laatste experiment lag de focus op de speenprocedure binnen het groepskraamsysteem (Hoofdstuk 6), aangezien speenproblemen niet alleen gerelateerd zijn aan huisvestingscondities voor en na spenen, maar ook aan de timing en abruptheid van het spenen zelf. Twee groepen groepskraambiggen, die een verschillende behandeling ondergingen tussen 4 en 9 weken leeftijd, 
werden gevolgd van geboorte tot slacht. Tussen 0 en 4 weken leeftijd werden beide groepen in het groepskraamsysteem gehuisvest. Daarna werd de ene groep abrupt gespeend en in een groep van circa 35 biggen gehuisvest in een opfokhok. De andere groep onderging een meer geleidelijke speenprocedure gedurende een verlengde zoogperiode van 9 weken. De procedure bestond uit verplichte 'intermittent-suckling' (IS) tijdens de vijfde lactatieweek (in deze periode werden de zeugen gescheiden van de biggen gedurende een aaneengesloten periode van 10 uur per dag), gevolgd door een periode van 4 weken waarin de zeugen zich vrijwillig konden afzonderen van de biggen. De toepassing van verplichte IS (in combinatie met beercontact) had ook als doel om bronst en ovulatie op te wekken, zodat de zeugen tijdens de zoogperiode geïnsemineerd konden worden. Op deze manier kan het reproductieniveau van de zeugen mogelijk op peil gehouden worden, ondanks de verlengde zoogperiode. Op 9 weken leeftijd werden beide groepen varkens overgeplaatst naar een vleesvarkensstal, waar ze gehuisvest werden onder gelijke omstandigheden. Het abrupte spenen op 4 weken leeftijd leek meer impact te hebben dan verplichte IS gevolgd door vrijwillige afzondering door de zeugen; binnen de eerste 2 weken na de start van de verschillende speenprocedures (van 4 tot 6 weken leeftijd), lieten de abrupt gespeende biggen een vermindering in dagelijkse groei zien en een duidelijke piek in 'belly-nosing' gedrag (met een 8 keer hogere frequentie van dit gedrag op 41 dagen leeftijd). Deze veranderingen waren niet te zien bij de groep biggen die nog in het groepskraamsysteem verbleef. Verder was het percentage biggen met pasteuze of waterige mest 2 keer zo hoog in de groep gespeende biggen. Voor de groep biggen die werd gespeend op 9 weken leeftijd, resulteerde spenen echter niet in een groeidip of in het vertonen van gedrag dat aan zou kunnen geven dat de biggen moeite hadden met de situatie na spenen. Daarbij was de voeropname na de overplaatsing naar de vleesvarkensstal vergelijkbaar in beide behandelingsgroepen, wat aangeeft dat de biggen die volgens de meer geleidelijke procedure waren gespeend een betere overgang hadden naar een dieet bestaande uit vast voer.

Ook op de langere termijn vonden we nog verschillende positieve effecten van de behandeling met de verlengde zoogperiode op het gedrag van de varkens, hoewel effecten op productiekenmerken verdwenen tijdens de vleesvarkensfase. 'Belly-nosing' werd vertoond op hogere niveaus tijdens de vleesvarkensfase door biggen die waren gespeend op 4 weken leeftijd, terwijl 
biggen die op 9 weken waren gespeend dit gedrag bijna niet vertoonden (de frequentie varieerde van 4 tot 16 keer hoger over de 3 observatiedagen tijdens de vleesvarkensfase). Daarbij hadden de biggen die op 9 weken waren gespeend minder huidbeschadigingen (gerelateerd aan agressie en beschadigend gedrag) in de tiende week van de vleesvarkensfase. Dit werd ondersteund door een lagere frequentie van beschadigend gedrag vanaf 4 weken leeftijd tot in de vleesvarkensfase, in vergelijking met de abrupt gespeende dieren $(1.2 \pm 0.2$ vs. $1.8 \pm 0.2$ keer per uur, $P<0.05)$. Mogelijk is dit een reflectie van de soepelere overgangen rond 4 en 9 weken leeftijd, voor de biggen die gespeend werden volgens de meer geleidelijke procedure.

Concluderend ondersteunen onze resultaten de hypothese dat het groepskraamsysteem bigontwikkeling zou bevorderen, vergeleken met gangbare huisvesting. Onze resultaten ondersteunen ook de hypothese dat binnen het groepskraamsysteem bigontwikkeling bevorderd wordt door een meer geleidelijk speenproces tijdens een verlengde zoogperiode, vergeleken met abrupt spenen op 4 weken leeftijd. We vonden verschillen in bigontwikkeling vóór spenen, rond overgangsperiodes rondom spenen, en in het latere leven. Deze verschillen werden weerspiegeld in gedrag, huidbeschadigingen, productiekenmerken, en aspecten van darmfunctie. Uitval van biggen vóór spenen was echter een probleem in het groepskraamsysteem. Dit was waarschijnlijk het gevolg van een suboptimaal kraamhok in combinatie met het gebruik van een type zeug dat mogelijk minder geschikt is voor alternatieve kraamsystemen zoals het groepskraamsysteem. In het algemeen lijken geleidelijke overgangen in huisvesting en management belangrijk voor een goede prestatie van zeugen en biggen. Het groepskraamsysteem kan bijdragen aan het verminderen van gangbare speenproblemen door i) biggen beter voor te bereiden op het moment van spenen door tijdens de kraamfase een meer stimulerende sociale en fysieke omgeving te bieden, ii) het verminderen of wegnemen van gelijktijdige stressoren bij spenen, en iii) het speenproces meer geleidelijk te laten verlopen tijdens een verlengde zoogperiode. 


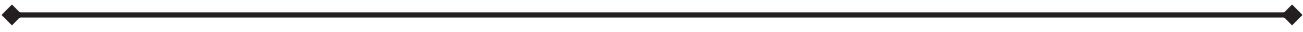

Acknowledgements

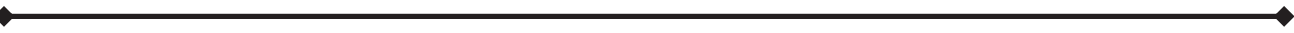


Een vreemd idee dat het alweer 5 jaar geleden is dat ik ben begonnen met het onderzoek naar de ontwikkeling van een nieuw groepshuisvestingssysteem voor kraamzeugen en hun biggen... Gaandeweg zijn er veel mensen bij het project betrokken geweest, die hebben bijgedragen aan de totstandkoming van dit proefschrift, en die een extra bedankje verdienen.

Allereerst Liesbeth Bolhuis en Nicoline Soede. Wat heb ik een geluk gehad met jullie als dagelijkse begeleiders! Jullie stonden altijd voor me klaar en gaven altijd positieve ondersteuning. Zelfs in moeilijkere tijden zijn jullie zeer betrokken gebleven en kon ik nog steeds bij jullie terecht. Heel veel dank voor al jullie gedetailleerde en waardevolle feedback, voor jullie positieve instelling, en voor het delen van jullie kennis. Daarnaast was het ook fijn dat jullie, naast alle werkgerelateerde aspecten, ook aandacht hebben voor hoe iemand is als persoon. En: hoe druk jullie ook waren, ik kon altijd even binnenlopen als ik ergens mee zat. Dat laatste geldt ook voor Bas Kemp. Ik voelde me altijd welkom om bij je aan te kloppen, en ik heb dankbaar gebruik gemaakt van jouw besluitvaardige vermogen om 'even een knoop door te hakken'.

Ik ben jullie drieën ook dankbaar dat jullie me de kans hebben gegeven om te werken aan dit geweldige project! Het sloot precies aan bij waar ik naar op zoek was: het doen van onderzoek dat verband houdt met gedrag en welzijn van varkens. Het was erg leuk om vanaf het begin betrokken te zijn bij het project en mee te denken over het ontwerp en management van het systeem, tot de praktische waarnemingen en het schrijven van papers aan toe. En wat is het heerlijk om je werk af en toe te kunnen afwisselen met biggetjes knuffelen!

Daarnaast mijn dank aan de anderen in het projectteam 'Groepshuisvesting kraamzeugen', bij nog steeds gebrek aan een betere naam ;-). Als eerste Carola van der Peet-Schwering. Als projectleider ben je bij alle mogelijke aspecten van het project betrokken geweest. Bedankt voor jouw gevarieerde bijdrage, van 'bemiddelen' tijdens soms hectische vergaderingen, tot het afstemmen van praktische zaken, en feedback geven op mijn papers. Verder bedankt aan Anita Hoofs, met name voor al je praktische inzichten die hebben bijgedragen aan de ontwikkeling van het systeem. Daarnaast zijn er vanuit VIC Sterksel verschillende innovatiemanagers betrokken geweest (Patrick Classens, Linda Troquet, en Loes van de Pas), met een soms lastige rol tussen 'de onderzoekers' en 'de dierverzorgers' in. Veel dank voor jullie inzet binnen het 
project! Als laatste ook bedankt aan Gisabeth Binnendijk, die met name in de laatste fase van het project onmisbaar was voor het aanleveren van gegevens vanuit VIC Sterksel.

Natuurlijk is een goede verzorging van de dieren ook essentieel voor een goed verloop van een experiment, en daarvoor mijn dank aan alle dierverzorgers vanuit VIC Sterksel (met name Pim Peters en Rob Nijssen) en Carus (vooral Ben van den Top en Rinie Ernste). Ook veel dank aan de overige medewerkers van VIC Sterksel en Carus die hebben bijgedragen aan het succes van de experimenten!

Verder heb ik veel hulp gehad van OBP'ers en studenten met het verzamelen van data. In het bijzonder heel veel dank aan Bjorge Laurenssen en Fleur Bartels. Jullie zijn onmisbaar geweest voor een goede voortgang van het onderzoek! Geen enkel experiment verloopt vlekkeloos, maar dankzij jullie ervaring, die van onschatbare waarde is, kwam alles altijd goed. Jullie hebben op allerlei manieren ondersteuning geboden met betrekking tot het opzetten en uitvoeren van praktisch werk, en het was heel fijn dat ik op jullie kon vertrouwen om een oogje in het zeil te houden. Ook wil ik graag Monique Ooms, Jan Wijnen, Ger de Vries Reilingh, Mike Nieuwland, en Rudie Koopmanschap bedanken voor hun hulp bij o.a. het verzamelen van gegevens, het bieden van technische ondersteuning, en het uitvoeren van labanalyses. Verder heb ik met het praktische werk het geluk gehad dat er veel studenten geïnteresseerd waren in het project. Anne-Riet Boerenkamp, Ilse de Bar, Annemarije Verspuy, Miranda Elling-Staats, Peggy Friesen, Anouk Welbers, Claire Walbecque, Sanne Benjamins, Jasmijn Strous, Nick Timmermans, Hilde de Kok, en Eline Burgers: bedankt voor jullie inzet met het verzamelen van waarnemingen! Naast de studenten die ik (mede) heb mogen begeleiden, ook dank aan alle andere studenten die betrokken zijn geweest bij het project.

I have been fortunate enough to work in a chair group with a great working atmosphere. First, a big thank you to my dear (ex) tropical roommates! Novi Mayasari, Ampai Nangsuay, Juncai Chen, Carol Souza da Silva, Danny de Koning, Carolina Lima Alvares da Silva, Tom Berghof, and Conny Maatjens. You made work a lot more fun! We had so many good times together: international dinners, drinks, excursions, parties, talks during the coffee breaks, walks during lunch time etc. Thank you especially to my dear Novi and Ampai 
for all your support, during all the good times but also during more difficult times. I really appreciate your friendship a lot. Also thanks to the newest members of the tropical room: Lu Luo, Francesca Marcato, and Wei Xu. Thank you so much for being my paranymphs and helping with taking pictures during my defence! Lastly, Jenny Soellner and Paulina Krzysica: you had a short stay at the ADP group, but it was a lot of fun to have you around!

Ook buiten het tropische klimaat van de tropical room zijn er veel mensen waar ik het gezellig mee heb gehad, waar ik veel van heb geleerd, en die mij hebben geholpen. De dames van het secretariaat (Lora van der Kleijn en Nanette van Hapert, met jullie talent om alles zo te regelen dat alles vlekkeloos verloopt), post-docs (thanks to Caroline Clouard and Inonge Reimert for your help with data collection and student supervision), andere staf van ADP (o.a. Henry van den Brand, Henk Parmentier, en Aart Lammers: bedankt voor het geven van feedback op mijn papers/meedenken over het onderzoek) en vele (voormalig) mede-PhD'ers. Allemaal bedankt voor de leuke tijd!

Als laatste wil ik mijn familie bedanken voor hun steun en interesse in mijn werk. In het bijzonder wil ik mijn lieve ouders bedanken, die altijd in mij geloven, altijd voor me klaarstaan, en mij onvoorwaardelijk steunen. Een hele grote knuffel voor jullie! 


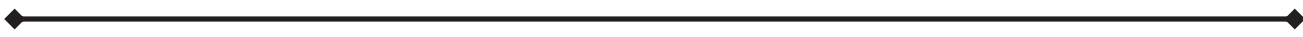

About the author 


\section{Curriculum Vitae}

Sofie Elise van Nieuwamerongen was born on March $14^{\text {th }} 1988$ in Utrecht, The Netherlands. After obtaining her high school VWO diploma at the Pallas Athene College in Ede, she pursued her interest in animals by studying Animal Sciences at Wageningen University \& Research. Her BSc thesis focussed on performing a welfare assessment of zoo animals, which included a behavioural study with tigers and lions. Her interest in pigs was evoked during her MSc studies, during which she specialised

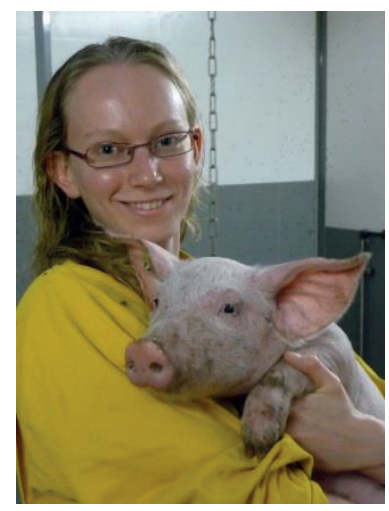
in 'Animal Health and Behaviour'. She performed her MSc thesis at the Adaptation Physiology Group, for which she studied effects of social breeding value and coping style on pig behaviour. To conclude her MSc studies, Sofie did an internship at 'Buitengewone Varkens', for which she observed the behaviour of pigs living in a natural environment. After obtaining her MSc degree, Sofie stayed at the Adaptation Physiology Group to work on a project on group housing systems for lactating sows and their piglets. During her ensuing $\mathrm{PhD}$ candidateship, she focussed more specifically on the development of the pigs raised in the newly designed 'multi-suckling' system. The results of this $\mathrm{PhD}$ research are presented in this thesis.

\section{Publications}

\section{Refereed scientific journals}

S.E. van Nieuwamerongen, N.M. Soede, C.M.C. Van der Peet-Schwering, B. Kemp and J.E. Bolhuis (2015). Development of piglets raised in a new multilitter housing system vs. conventional single-litter housing until 9 weeks of age. Journal of Animal Science 93(11): 5442-5454.

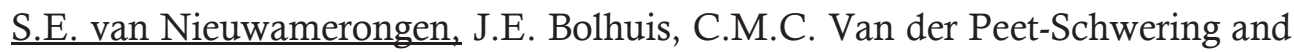
N.M. Soede (2014). A review of sow and piglet behaviour and performance in group housing systems for lactating sows. Animal 8(3): 448-460. 


\section{Conference book of abstracts}

S.E. van Nieuwamerongen, J.E. Bolhuis, C.M.C. Van der Peet-Schwering, B. Kemp and N.M. Soede (2016). Piglets' peri-weaning intestinal functioning: a multi-suckling system vs. farrowing crates. Abstract in Book of abstracts of the $16^{\text {th }}$ International Conferences on Production Diseases in Farm Animals (ICPD), page 66. Wageningen, The Netherlands, 20-23 June 2016.

B.F.A. Laurenssen, J.E.M. Strous, S.E. van Nieuwamerongen, J.E. Bolhuis, C.M.C. van der Peet-Schwering and N.M. Soede (2016). Lactational oestrus in group housed lactating sows. Abstract in Book of abstracts of the $16^{\text {th }}$ International Conferences on Production Diseases in Farm Animals (ICPD), page 165. Wageningen, The Netherlands, 20-23 June 2016.

S.E. van Nieuwamerongen, J.E. Bolhuis, C.M.C. van der Peet-Schwering and N.M. Soede (2016). Development of piglets raised in either a multi-litter system or a single-litter system. Abstract in Proceedings of the WIAS Science Day 2016, page 16. Wageningen, The Netherlands, 4 February 2016.

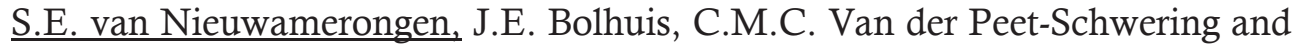
N.M. Soede (2015). Post-weaning performance of piglets raised in a multisuckling system vs. farrowing crates. Abstract in Book of abstracts of the $66^{\text {th }}$ Annual Meeting of the European Federation of Animal Science (EAAP), page 540. Warsaw, Poland, 31 August-4 September 2015.

I. Reimert, T. van Maurik, S.E. van Nieuwamerongen, C.M.C. van der PeetSchwering and J.E. Bolhuis (2015). Friends forever? Inter-individual distances in group housed lactating sows. Abstract in Book of abstracts of the $34^{\text {th }}$ International Ethological Conference (ICE), Australasian Society for the Study of Animal Behaviour (ASSAB), Australasian Evolution Society (AES), and Australasia, New Zealand and Africa Region of Applied Ethology, Cairns, Australia, 9-14 August 2015.

S.E. van Nieuwamerongen (2015). Growing up in a large community improves performance and welfare of piglets. Abstract in Proceedings of the Wageningen $\mathrm{PhD}$ symposium 'Connecting ideas, combining forces', page 2. Wageningen, The Netherlands, 6 May 2015. 


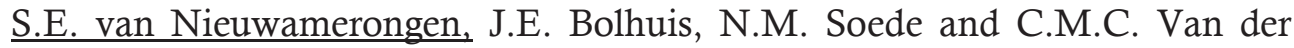
Peet-Schwering (2015). Developing a group housing system for lactating sows and their litters. Abstract in Proceedings of the International Pig Welfare conference (IPW) 'Improving pig welfare - what are the ways forward?', page 139. Copenhagen, Denmark, 29-30 April 2015.

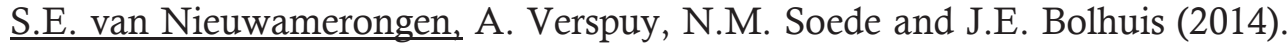
Behavioural development of piglets in farrowing crates and in a multi-suckling system. Abstract in Proceedings of the $48^{\text {th }}$ International Congress of the International Society for Applied Ethology (ISAE), page 283. Vitoria-Gasteiz, Spain, 29 July-2 August 2014.

\section{Other publications}

C.M.C. Van der Peet-Schwering, S.E. van Nieuwamerongen, J.E. Bolhuis, G.P. Binnendijk and N.M. Soede (2017). Groepskraamsysteem: ontwikkeling van zeugen, biggen en vleesvarkens bij spenen op 4 of 9 weken leeftijd. Livestock Research Rapport 1012, Wageningen University \& Research.

C.M.C. Van der Peet-Schwering, S.E. van Nieuwamerongen, J.E. Bolhuis, L.M.P. Troquet, A. Hoofs and N.M. Soede (2015). Groepskraamsysteem: analyse van de ontwikkeling van zeugen, biggen en vleesvarkens. Livestock Research Rapport 880, Wageningen University \& Research.

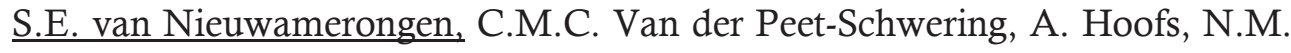
Soede and J.E. Bolhuis (2014). Succes- en risicofactoren van groepshuisvesting van kraamzeugen. Livestock Research Rapport 757, Wageningen UR Livestock Research.

S.E. van Nieuwamerongen (2014). Reviewing group housing for lactating sows. Blog. http://blog.journals.cambridge.org/2014/03/reviewing-group-housingfor-lactating-sows/ 


\section{WIAS Training and Supervision Plan ${ }^{1}$}

\section{Description}

Year

The basic package (3.0 ECTS)

'Introduction course' (WIAS), Wageningen, The Netherlands

2014

Course 'Ethics and philosophy in life sciences' (WIAS),

Wageningen, The Netherlands

2014

\section{Disciplinary competences (16.5 ECTS)}

Writing a literature review and WIAS proposal

Course 'Adaptive animals and livestock farming systems to meet

global changes' (Agreenium and WUR), Rennes, France

2014

Course 'Interpretation of animal stress responses' (Aarhus

University), Bredsten, Denmark

Course 'Statistics for the life sciences' (WIAS), Wageningen, The

Netherlands

'Advanced statistics course: Design of experiments' (WIAS),

Wageningen, The Netherlands

Participation in discussion group 'Animal health and immunology'

(WIAS), Wageningen, The Netherlands

\section{Professional competences (10.4 ECTS)}

Course 'The essentials of scientific writing and presenting'

(Wageningen in'to Languages), Wageningen, The Netherlands

Member of the organising committee of the 'WIAS Science Day

2015' (WIAS), Wageningen, The Netherlands

Course 'Career orientation' (WGS), Wageningen, The Netherlands

Course 'Project and time management' (Valley Consult),

Wageningen, The Netherlands

Course 'Theatre skills for lecturers' (ESD), Wageningen, The

Netherlands

Course 'Presenting with impact' (Wageningen in'to Languages),

Wageningen, The Netherlands

Course 'Interpersonal communication for PhD students' (WGS),

Wageningen, The Netherlands

Course 'Effective behaviour in your professional surroundings'

(WGS), Wageningen, The Netherlands 
Course 'Last stretch of the $\mathrm{PhD}$ programme' (WGS), Wageningen, The Netherlands

Course 'The final touch: writing the general introduction and discussion' (WIAS), Wageningen, The Netherlands

\section{Presentation skills (4.0 ECTS)}

ISAE conference poster presentation, Vitoria-Gasteiz, Spain

'Improving pig welfare- what are the ways forward?' conference oral presentation, Copenhagen, Denmark

'Wageningen PhD symposium' oral presentation, Wageningen, The Netherlands

CAWA meeting oral presentation, Wageningen, The Netherlands 2015

EAAP conference oral presentation, Warsaw, Poland 2015

'WIAS science day' symposium oral presentation, Wageningen, The Netherlands

ICPD conference oral presentation, Wageningen, The Netherlands

\section{Teaching competences (6.0 ECTS)}

Supervising 10 MSc students (WUR)

Supervising project group for 'Sector integration course: pigs and poultry' (WUR)

Giving a lecture for 'Sector integration course: pigs and poultry' (WUR)

${ }^{1} 1$ ECTS credit equals a study load of approximately 28 hours 

The first 3 years of research described in this thesis were conducted as part of the public-private partnership 'Samenwerkende Varkenshouderijketen', with funding from the topsector 'Agri\&Food'. The last year of research described in this thesis was funded by the Dutch Ministry of Economic Affairs.

Cover design:

Thesis printed by:
S.E. van Nieuwamerongen

ProefschriftMaken | Digiforce,

Vianen, The Netherlands 